\title{
Endothelial dysfunction and low-grade inflammation : determined by diet and cause of arterial stiffness
}

Citation for published version (APA):

van Bussel, B. C. T. (2014). Endothelial dysfunction and low-grade inflammation : determined by diet and cause of arterial stiffness. [Doctoral Thesis, Maastricht University]. Maastricht University. https://doi.org/10.26481/dis.20140528bb

Document status and date:

Published: 01/01/2014

DOI:

10.26481/dis.20140528bb

Document Version:

Publisher's PDF, also known as Version of record

\section{Please check the document version of this publication:}

- A submitted manuscript is the version of the article upon submission and before peer-review. There can be important differences between the submitted version and the official published version of record.

People interested in the research are advised to contact the author for the final version of the publication, or visit the DOI to the publisher's website.

- The final author version and the galley proof are versions of the publication after peer review.

- The final published version features the final layout of the paper including the volume, issue and page numbers.

Link to publication

\footnotetext{
General rights rights.

- You may freely distribute the URL identifying the publication in the public portal. please follow below link for the End User Agreement:

www.umlib.nl/taverne-license

Take down policy

If you believe that this document breaches copyright please contact us at:

repository@maastrichtuniversity.nl

providing details and we will investigate your claim.
}

Copyright and moral rights for the publications made accessible in the public portal are retained by the authors and/or other copyright owners and it is a condition of accessing publications that users recognise and abide by the legal requirements associated with these

- Users may download and print one copy of any publication from the public portal for the purpose of private study or research.

- You may not further distribute the material or use it for any profit-making activity or commercial gain

If the publication is distributed under the terms of Article $25 \mathrm{fa}$ of the Dutch Copyright Act, indicated by the "Taverne" license above, 
Endothelial dysfunction and low-grade inflammation: determined by diet and cause of arterial stiffness 


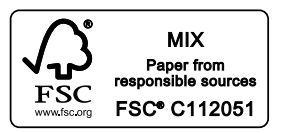

Copyright @ B.C.T. van Bussel, Maastricht 2014

No part of this book may be reproduced or transmitted in any form or by any means, without prior permission in writing by the author, or when appropriate, by the publishers of the publications.

Layout: Tiny Wouters

Cover Design: Jean Scheijen (www.vierdrie.nl)

Production: Ipskamp Drukkers, Enschede

ISBN: 978-94-6259-164-6

The study presented in this thesis was performed within the framework of TI Food and Nutrition

\section{Nutrition and Health}

Research on nutrition and health integrates laboratory and clinical studies to identify food components that affect the risk of cardiovascular disease, cancer and infectious disease. There is need to understand the mode of action of such food components in relation to the risk of these common diseases, which offer good prospects for nutrition intervention. Research projects may start from either the laboratory or the clinical side but should involve both approaches.

\section{Diet and early changes of endothelial function}

Cardiovascular disease (CVD) is the leading cause of death and disability in Western society. It is caused by undesired changes in the functioning of the inner wall of blood vessels: the endothelium. These changes lead to complications such as arterial stiffening. Diet and lifestyle can both induce and help prevent these changes. In the project Diet and early endothelial dysfunction we have investigated the role of diet on early changes in vascular function and we have identified diet sensitive biomarkers of cardiovascular health. Mechanistically validated biomarkers will ultimately support the food industry in the development of foods with well-substantiated health claims, approved by authorities such as the European Food Safety Authority (EFSA). 


\title{
Endothelial dysfunction and low-grade inflammation: determined by diet and cause of arterial stiffness
}

\author{
PROEFSCHRIFT \\ ter verkrijging van de graad van Doctor aan de Universiteit Maastricht, \\ op gezag van de Rector Magnificus, \\ Prof. dr. L.L.G. Soete \\ volgens het besluit van het College van Decanen, \\ in het openbaar te verdedigen \\ op woensdag 28 mei 2014 om 16:00 uur
}

door

Bas Carolus Theodorus van Bussel Geboren 2 februari 1983, te Asten-Heusden 
Promotores:

Prof. dr. C.D.A. Stehouwer

Prof. dr. C.G. Schalkwijk

\section{Copromotor:}

Dr. R.M.A. Henry

\section{Beoordelingscommissie:}

Prof. dr. ir. R.P. Mensink, voorzitter

Prof. dr. M.L. Bots, Universiteit Utrecht

Prof. dr. ir. P.C. Dagnelie

Prof. dr. P.W. de Leeuw

Prof. dr. N.C. Schaper

Financial support by the Dutch Heart Foundation for the publication of this thesis is gratefully acknowledged. In addition, the publication of this thesis was financially supported by Esaote B.V., which is also gratefully acknowledged. 
Voor pap en mam 


\section{Contents}

$\begin{array}{lll}\text { Chapter } 1 \text { General introduction } & 9\end{array}$

Part I Methodology of biomarker detection 29

Chapter 2 Multiple inflammatory biomarker detection in a prospective 31 cohort study: a cross-validation between well-established single-biomarker techniques and an electrochemiluminescencebased multi-array platform

Chapter 3 Control systems approach to assessment of flow-mediated dilation improves the detection of changes in local brachial artery dilation after an oral glucose load

Part II Diet and endothelial dysfunction and low-grade inflammation 75

Chapter 4 Fish consumption in healthy adults is associated with decreased circulating biomarkers of endothelial dysfunction and inflammation during a 6-year follow-up

Chapter 5 Alcohol and red wine consumption are associated with less endothelial dysfunction and less low-grade inflammation: the Hoorn Study

Chapter 6 A healthy diet is associated with less endothelial dysfunction and less low-grade inflammation over a 7-year period: the CODAM Study

Chapter 7 Unhealthy dietary patterns associated with inflammation and endothelial dysfunction in type 1 diabetes

Part III Endothelial dysfunction, low-grade inflammation and arterial stiffness

Chapter 8 Endothelial dysfunction and low-grade inflammation are associated 161 with greater arterial stiffness over a 6-year period

Chapter 9 Low-grade inflammation, but not endothelial dysfunction, is associated with greater carotid stiffness in the elderly: the Hoorn Study 
Summary (samenvatting voor de leek)

Acknowledgements (dankwoord)

239

Curriculum vitae

List of publications 

Chapter 1

Introduction 
Chapter 1

1 


\section{Introduction}

The vascular endothelium consists of a monolayer of endothelial cells lining the inner blood vessel wall and is the primary site of dysfunction in many diseases, particularly in cardiovascular disease (CVD) ${ }^{1-6}$ The vascular endothelium interacts with inflammatory cells of the immune system. ${ }^{3-6}$ Endothelial dysfunction and low-grade inflammation, which are thus closely related ${ }^{4,7}$, mediate the initiation and the progression of CVD and are potential targets for the prevention and/or reduction of CVD. ${ }^{1-6}$ Paragraph 1.1 introduces the vascular endothelium, endothelial dysfunction and low-grade inflammation in more detail.

Diet is perhaps one of the most modifiable CVD risk factors. ${ }^{8} \mathrm{~A}$ healthy diet has been associated with a reduced incidence of CVD. ${ }^{9-14}$ Endothelial dysfunction and low-grade inflammation might be mechanisms through which a healthy diet may prevent and/or reduce CVD. The present thesis therefore investigates the associations between diet and endothelial dysfunction and low-grade inflammation in multiple populations without and with existing CVD. Paragraph 1.2 describes in more detail how diet is assessed.

Endothelial dysfunction and low-grade inflammation have been associated with (incident) CVD. ${ }^{15-30}$ In part, these associations can be explained by the fact that endothelial dysfunction and low-grade inflammation play key roles in atherothrombosis. ${ }^{3-6}$ However, other mechanisms that have been associated with (incident) CVD, such as arterial stiffening ${ }^{31-33}$, could also be involved. The present thesis therefore investigates the associations between, on the one hand, endothelial dysfunction and low-grade inflammation and, on the other, arterial stiffness. The concept of arterial stiffness is explained in more detail in paragraph 1.3.

Finally, this chapter briefly introduces the study populations in paragraph 1.4 and presents the aim and outline of this thesis in paragraph 1.5 .

\section{The vascular endothelium, endothelial dysfunction and low- grade inflammation (1.1)}

\section{The vascular endothelium}

The vascular endothelium is a large heterogeneous organ, totaling 1-6 × $10^{13}$ cells, that responds to numerous autocrine, paracrine and physiological stimuli (e.g. chemo and cytokines, hormones, pulsatile stress, shear stress, blood flow and oxygen partial pressure $)^{34}$ and performs many essential physiological functions throughout the vasculature, including regulation of vasomotor tone, vascular cell proliferation and survival, blood cell trafficking, barrier function, haemostatic balance and inflammation. ${ }^{1,2}$ Particular responses and functions of the vascular endothelium differ per site and each endothelial cell within the vascular endothelium is therefore 
phenotypically adapted in a way to optimally serve its surrounding tissue's needs. ${ }^{1,2,34}$ For example, a certain degree of permeability in the glomeruli is of utmost importance for kidney function, whereas a highly selective and largely impermeable endothelial barrier in the cerebral vasculature is essential for brain function. These and other phenotypic adaptations, thus, depend on the physiological context of endothelial cells. $^{1,2,34}$ The heterogeneous nature of the vascular endothelium needs to be considered when investigating endothelial dysfunction.

\section{Endothelial dysfunction}

Endothelial dysfunction can be defined as any change in endothelial responses and/or functions that is inappropriate with regard to the preservation of organ function. ${ }^{35}$ Endothelial dysfunction, thus, has many dimensions. The phenotypic specificity explained above, in particular, makes endothelial cells extremely sensitive to various pathological changes that may initiate and aggravate (cardiovascular) disease. ${ }^{1,2,34}$ Generalized endothelial dysfunction (i.e. affecting many dimensions), therefore, is considered an important player in the initiation and progression of CVD. ${ }^{35-37}$

\section{Measurements of endothelial dysfunction}

Endothelial dysfunction can be measured in many ways due to the heterogeneous nature of the vascular endothelium. ${ }^{34,37-45}$ Both circulating biomarkers detectable in plasma or serum, and non-invasive functional biomarkers are, particularly, useful to study generalized endothelial dysfunction in population-based studies that seek to relate lifestyle to CVD risk factors ${ }^{37}$, while additional controlled laboratory experiments that study the specific (intra-)cellular pathways involved in endothelial dysfunction are complementary to our understanding. ${ }^{1,2}$

We used an extensive multi-marker approach to characterize endothelial dysfunction over a broad range of domains in the populations studied, thereby aiming to account for the heterogeneous nature of the vascular endothelium, while others primarily focussed on single biomarkers. ${ }^{34,37-44,46-49}$ Vascular cell adhesion molecule 1 (sVCAM-1), intercellular adhesion molecule 1 (sICAM-1), endothelial selectin (sE-selectin) and thrombomodulin (STM) are expressed by endothelial cells and soluble forms are shed into the circulation after cleavage at the cell membrane through a biological process called proteolysis. ${ }^{1-6}$ In addition, the glycoprotein von Willebrand factor (vWf) is synthesized by endothelial cells and released upon their activation or injury. ${ }^{1-6}$ Flow-mediated dilation (FMD), particularly of the brachial artery, is a functional biomarker that is measured by ultrasonography and is often used in population-based studies. ${ }^{46-49}$ Basically, FMD quantifies the capacity of the vascular endothelium to induce arterial vasodilation, which is evoked through an ischaemia-induced blood flow increase (i.e. hyperaemia). ${ }^{46-49}$ Vasodilation and/or flow increase are thought to reflect endothelial synthesis of vasomotor mediators, such as nitric oxide, which is an important mediator in vascular health because it drives 
vasodilation and has anti-platelet, anti-proliferative, anti-adhesive, permeabilitydecreasing and anti-inflammatory properties. ${ }^{35,46-49}$

However, measurement of FMD is complex and its limitations are currently discussed in the literature. ${ }^{50}$ Nevertheless some authors deem functional biomarkers such as FMD the most important measure of endothelial dysfunction, as opposed to circulating biomarkers. $^{43}$ Importantly, both higher concentrations of sVCAM-1, sICAM-1, sE-selectin, STM and VWf, and a lower FMD have been associated to (incident) CVD. ${ }^{15-25,51,52}$ With the fact that each of the circulating biomarkers primarily originates from the vascular endothelium it is reasonable to assume that both higher concentrations of circulating biomarkers and a lower FMD represent endothelial dysfunction. $^{37}$

\section{Low-grade inflammation}

Inflammation is a reaction of the body to injury or infection and, in particular, lowgrade inflammation plays a role in atherothrombosis by driving the interaction between inflammatory cells and the vascular endothelium. ${ }^{3-6}$ Low-grade inflammation and endothelial dysfunction are thus closely related. ${ }^{4,7}$ Low-grade inflammation is a multi-faceted process, characterized by an upregulation of inflammatory proteins and cytokines, such as C-reactive protein (CRP), serum amyloid A (SAA), interleukin 6 (IL-6), interleukin 8 (IL-8), tumour necrosis factor $\alpha$ (TNF- $\alpha$ ) and SICAM-1. ${ }^{3-6}$ sICAM-1 is expressed on both the vascular endothelium and monocytes and therefore considered a biomarker of both endothelial dysfunction and low-grade inflammation. ${ }^{53}$ Higher circulating concentrations of these six molecules (within the normal range excluding acute injury or infection) have been associated with (incident) CVD. ${ }^{15,16,18-20,22-30}$ Therefore, these six circulating biomarkers were investigated in the present thesis and can be considered a fair, although not exhaustive $34,37,54-59$, estimate of low-grade inflammation, particularly when compared to the number of biomarkers used to estimate low-grade inflammation by others. $34,37,54-59$

\section{Summary}

In an attempt to investigate the vascular endothelium as one integrated organ, while taking its heterogeneity into account, the present thesis focuses on generalized endothelial dysfunction with the use of a conceptual multi-marker approach, while concurrently addressing the closely related and multi-faceted process of low-grade inflammation (Figure 1.1). ${ }^{1-6}$ 


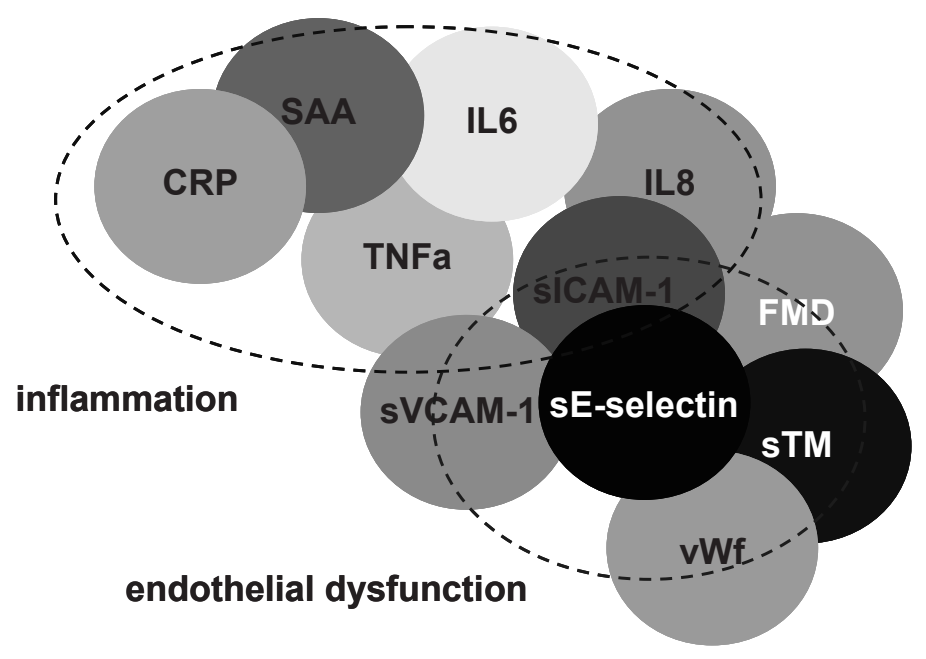

Figure 1.1 A conceptual presentation of multiple markers of endothelial dysfunction and low-grade inflammation; dashed circle represents endothelial dysfunction; dashed ellipse represents low-grade inflammation; CRP, C-reactive protein; SAA, serum amyloid A; IL-6, interleukin 6; TNF- $\alpha$, tumour necrosis factor alpha; IL-8, interleukin 8; sICAM-1, soluble intercellular adhesion molecule 1; FMD, flow-mediated dilation; sVCAM-1, soluble vascular cell adhesion molecule 1; sE-selectin, soluble endothelial selectin; sTM, soluble thrombomodulin; vWf, von Willebrand factor.

\section{Dietary determinants of endothelial dysfunction and low- grade inflammation (1.2)}

Improvement of diet is increasingly recognized as an important target for prevention of CVD in the general population and to reduce CVD in populations at risk. ${ }^{8,60}$ Endothelial dysfunction and low-grade inflammation might be mechanisms through which a healthy diet may prevent and/or reduce CVD.

Although several population-based studies on the association between diet and endothelial dysfunction and/or low-grade inflammation have been performed, their results are heterogeneous and impossible to directly compare because of the following reasons ${ }^{61-71}$ : (1) different (sets of) biomarkers were used ${ }^{61-71}$; (2) multiple ${ }^{61-66,68-71}$ versus single biomarkers were used ${ }^{67}$; (3) either endothelial dysfunction or low-grade inflammation was studied ${ }^{67}$; (4) results were reported for diet scores ${ }^{61-63}$ or individual food components only ${ }^{64-71}$; (5) results for individual food components were often poorly mutually adjusted for other food groups ${ }^{66-69} ;(6)$ and data were primarily cross-sectional. ${ }^{61-71}$ 
Evidence suggests that diet is stable over time ${ }^{72}$ and it is often presumed that results from cross-sectional studies can be extrapolated over time. However, longitudinal observational studies that confirm this presumption for the associations between diet and endothelial dysfunction and low-grade inflammation have not been reported.

The present thesis focused on the following five food groups (fish, vegetables, fruit, alcohol-containing beverages and dairy products), because food, instead of nutrients, is the principal unit in human nutrition. ${ }^{73}$ However, some data on food groups were unavailable and nutritional compounds (related to fish, vegetable, fruit, alcoholcontaining beverages and dairy products) were alternatively investigated. Although other potentially interesting dietary components exist that might affect endothelial dysfunction and low-grade inflammation ${ }^{74-84}$, the present thesis investigated the selected food groups for two reasons. First, previous population-based studies on diet scores / patterns have shown that healthy diets, rich in fish, fruit, vegetables, with alcohol-containing beverages and low in dairy products, are associated with lower circulating concentrations of biomarkers of endothelial dysfunction and low-grade inflammation. ${ }^{61-63}$ Second, such a healthy diet has been associated with a reduced (incidence of) CVD. ${ }^{9-14}$

For the reasons above, the present thesis aimed to investigate the associations between, on the one hand, fish, vegetable, fruit, alcohol-containing beverages and dairy product consumption and, on the other hand, extensive sets of biomarkers of both endothelial dysfunction and low-grade inflammation in several study populations with and without CVD and diabetes, using both cross-sectional and longitudinal designs, while consistently executing mutual adjustments for each of the other food groups (or nutrients).

We thus aimed to draw more firm conclusions whether or not a habitual healthy diet [and/or (which) specific food group(s)] is consistently associated with endothelial dysfunction and low-grade inflammation in health and disease.

\section{Dietary exposure assessment}

Dietary intake was either measured using the dietary history method, the Food Frequency Questionnaire (FFQ) or the food record method and each method has been described in detail elsewhere. ${ }^{85-93}$ Briefly, the dietary history method, which was developed by Burke, measures many details about characteristics of foods and primarily consists of an interview on the habitual pattern of eating, and with a food list on frequency and amount of intake, and a three-day dietary record as crosschecks. $^{85-87}$ The FFQ queries the frequency of consumption (and optionally portion size) of a finite list of food items over a specified period of time in the recent past, generally the previous year, and thereby ranks persons according to their dietary intake. ${ }^{85,86,88-91}$ The food record method is a detailed protocol of all foods and beverages consumed over a specific period of time, usually 3-7 days..$^{85,86,88,92,93}$ Each 
method has several advantages over the others. The FFQ, for example, has a (relatively) low time investment, low burden on respondents and investigators, low costs, and low chance of influence of altered eating behaviour. The dietary history method consists of an extensive dietary characterization, while using cross-checks that contribute to dietary assessment validity, and suffers low influence of day-to-day variability in diet. The food record method consists of an extensive dietary characterization by allowing numerous food items and does not require highly trained interviewers or highly cognitive performance of respondents. Importantly, each of these methods has repeatedly and successfully been used for dietary exposure assessment in large population-based studies. ${ }^{85,86}$

\section{Endothelial dysfunction, low-grade inflammation and arterial stiffening (1.3)}

Endothelial dysfunction and low-grade inflammation have been associated with (incident) $C V D^{15-30}$ and this association, in part, is mediated by the fact that endothelial dysfunction and low-grade inflammation play key roles in atherothrombosis. ${ }^{3-6}$ However, other mechanisms that have been associated with (incident) CVD, such as arterial stiffening ${ }^{31-33}$, could also be involved. ${ }^{94-98}$

Arterial stiffening causes CVD according to the following pathophysiological model that has been described extensively elsewhere. ${ }^{34,96-98,116}$ Briefly, each contraction of the heart propagates a pressure wave into the arterial circulation that travels forward until it meets an impedance mismatch [e.g. at the arterioles and branching arteries, due to the artery's anatomical taper or (calcified) plaques]. At these sites, the pressure wave is reflected and travels back towards the heart. When arteries are elastic, the backward pressure wave arrives at the heart in diastole, when the aortic valve is closed, and propagates extra blood into the coronary arteries. As arteries stiffen, the pressure waves (both forward and backward) travel faster throughout the arterial tree as compared to elastic arteries and the backward wave reaches the heart early, in systole. Diastolic blood pressure thereby decreases and no extra blood is propagated into the coronary arteries. Instead, the workload of the heart increases, because the backward pressure wave adds up to the forward pressure wave, increasing systolic blood pressure. This leads to an elevation of the cardiac afterload (if stroke volume is maintained). These alterations caused by stiffer arteries lead to decreased coronary perfusion, to increased left ventricular hypertrophy and to an increased risk of stroke. When these alterations progress, they eventually can culminate into myocardial infarction and heart failure. ${ }^{34,96-98,116}$

Endothelial dysfunction and low-grade inflammation may cause increased functional arterial stiffness through reduced nitric oxide availability and increased activity of vasoconstrictors, such as endothelin-1, that lead to vasoconstriction by vascular smooth muscle cells. ${ }^{94,95}$ On the other hand, endothelial dysfunction and low-grade 
inflammation may induce processes which involve the formation of cross-links, a shift from elastin towards relatively more collagen and the deposition of calcium on elastin that alter structural proteins in the arterial wall and thereby lead to increased structural arterial stiffness. ${ }^{96,97}$

Previous population-based studies that investigated this subject were crosssectional $^{99-115}$, most focused on either endothelial dysfunction ${ }^{113}$ or low-grade inflammation ${ }^{99,103-107,109,110,112,114,115}$, most included few biomarkers $99-107,109,110,112-115$ and did not investigate whether any relationship with arterial stiffening was interdependent $^{99-115}$, while endothelial dysfunction and low-grade inflammation are closely linked and difficult to separate conceptually. ${ }^{7}$

In light of the above, the present thesis investigated the associations between, on the one hand, endothelial dysfunction and low-grade inflammation and, on the other, arterial stiffness in both a cross-sectional and a longitudinal study design, focusing on extensive sets of biomarkers of endothelial dysfunction and low-grade inflammation and investigating, by mutual adjustments, whether any associations with arterial stiffening were interdependent.

We aimed thus to contribute evidence to the pathophysiological framework whether endothelial dysfunction and low-grade inflammation may cause arterial stiffening and thereby lead to CVD.

\section{Measurement of arterial stiffness}

Arterial stiffness can non-invasivally be determined either locally, at specific arterial sites (e.g. carotid, femoral, brachial), or regionally, over a given arterial segment (e.g. aorta). ${ }^{34,97,98,116}$

Local arterial stiffness is often expressed as distensibility coefficient (DC), compliance coefficient (CC), $\beta$-stiffness index (SI) or Young's Elastic Modulus (YEM). To calculate these parameters, the arterial diameter, distention and intima-media thickness are measured by means of ultrasonography ${ }^{34,97,98,116,117}$ and local pulse pressure is deducted using measured pressure wave forms according to the Van Bortel calibration method. ${ }^{118}$ A lower DC represents local arterial stiffness; a lower CC represents a lower arterial buffering capacity; a higher SI indicates local arterial stiffness and, in contrast to the other local measures, approximates the non-linear relationship between pressure and diameter; and a higher YEM represent higher intrinsic wall stiffness. Local stiffness of the carotid artery has been associated to a greater risk of stroke. $^{119}$

Regional arterial stiffness is often determined by pulse wave velocity (PWV), which is the travel speed of a pulse pressure wave, generated by the heart's contraction, over a given arterial segment. With ultrasonography, first, the travel time of the pulse pressure wave from the heart (as triggered by an electrocardiogram) to the distal end of the arterial segment is measured. Then, the measurement is repeated for the proximal end. Subtraction of the two travel times gives the transit time..$^{34,97,98,116}$ The 
transit time can also be measured directly with applanation tonometry, using two pressure sensors simultaneously applied at each end of the arterial segment. ${ }^{34,97,98,116}$ Next, the distance of the arterial segment is measured. PWV can then be calculated as the distance divided by the transit time. A faster PWV represents greater arterial stiffness and has been associated with (incident) CVD. ${ }^{33}$

\section{Study populations (1.4)}

The following four well characterized cohort studies, each extensively described elsewhere ${ }^{16,17,92,93,117,120-142}$, were investigated: The Amsterdam Growth and Health Longitudinal Study (AGAHLS) ${ }^{120-127}$, The Hoorn Study ${ }^{16,17,117,128-131}$, The Cohort on Diabetes and Atherosclerosis Maastricht (CODAM) Study ${ }^{131-136}$ and The EURODIAB Study. ${ }^{92,93,137-142}$

The present thesis investigated the associations between diet and endothelial dysfunction and low-grade inflammation in multiple settings. The AGAHLS enabled to investigate young healthy adults and to use longitudinal data over a 6-year period. The Hoorn Study included elderly individuals with CVD and diabetes mellitus type 2 (prevalences of 51.4 and $40.6 \%$, respectively) and the data were cross-sectional. The CODAM Study enabled to investigate the association between diet and endothelial dysfunction and low-grade inflammation over a 7-year period in older adults with and without CVD and diabetes mellitus type 2 (prevalences of 27.7 and $25.4 \%$, respectively). The EURODIAB Study included adults with a doctor's diagnosis of type 1 diabetes mellitus and enabled to investigate the associations between repeated dietary data over an 8-year period and biomarkers of endothelial dysfunction and lowgrade inflammation at the end of this period.

The associations between endothelial dysfunction and low-grade inflammation and arterial stiffness, in particular of the carotid artery, were investigated in both the AGAHLS and The Hoorn Study as both studies included data on arterial stiffness. 


\section{Aim and outline of the thesis (1.5)}

The main aim of the present thesis was to provide evidence whether endothelial dysfunction and low-grade inflammation are determined by diet and cause arterial stiffening (Figure 1.2).

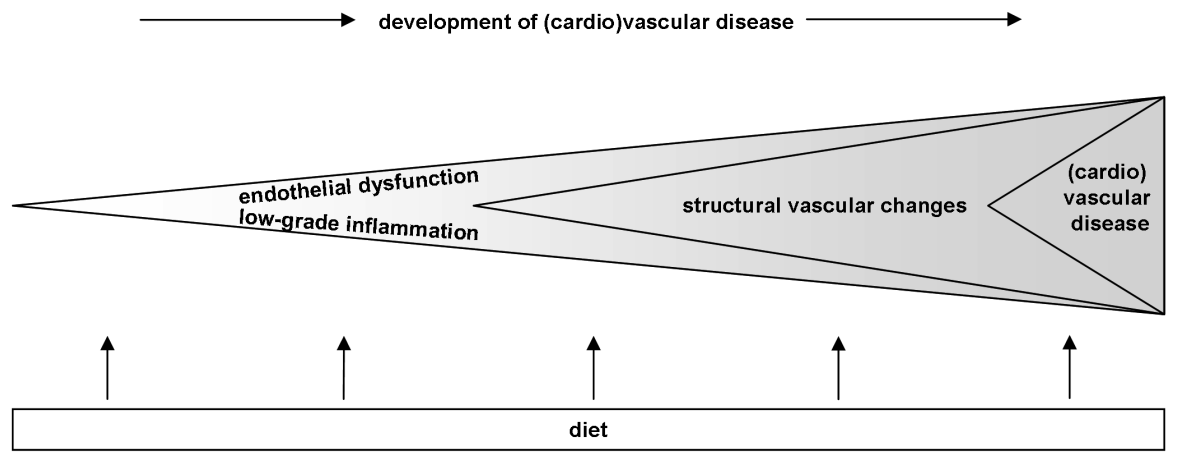

Figure 1.2 Pathophysiological framework for dietary effects on the initiation and progression of cardiovascular disease studied in the present thesis.

As endothelial dysfunction and low-grade inflammation are central in the present thesis, we focused on some methodological issues with regard to their measurements in Part 1 before addressing the main aim of the thesis. To employ a multi-marker approach in several cohort studies, we identified a new, more efficient, multipledetection method for circulating biomarkers and executed a cross-validation with well-established single-biomarker techniques (chapter 2). In addition, we highlight important aspects of FMD measurements investigating the role of flow increase in the quantification of vasodilation (chapter 3 ).

We continued by examining the associations between diet and endothelial dysfunction and low-grade inflammation in Part 2. We investigated whether a high consumption of fish, fruit and vegetables, and a low consumption of alcoholcontaining beverages and dairy products, were associated with less endothelial dysfunction and low-grade inflammation in healthy adults (chapter 4) and older adults/elderly individuals (chapters 5 and 6). Then, we re-investigated this topic in adults with type 1 diabetes mellitus, using dietary data on the nutrient compound level (chapter 7). 
In Part 3, we examined the associations between, on the one hand, endothelial dysfunction and low-grade inflammation and, on the other, arterial stiffness, in healthy adults (chapter 8) and elderly individuals (chapter 9).

Finally, we summarized and discussed the major findings of the present thesis and provided some leads for future research (chapter 10). 


\section{References}

1. Aird WC. Phenotypic heterogeneity of the endothelium: I. Structure, function, and mechanisms. Circ Res. 2007; 100:158-173.

2. Aird WC. Phenotypic heterogeneity of the endothelium: II. Representative vascular beds. Circ Res. 2007; 100:174-190.

3. Ross R. Atherosclerosis--an inflammatory disease. N Engl J Med. 1999; 340:115-126.

4. Borissoff JI, Spronk HM, ten Cate H. The hemostatic system as a modulator of atherosclerosis. N Engl J Med. 2011; 364:1746-1760.

5. Lusis AJ. Atherosclerosis. Nature. 2000; 407:233-241.

6. Hansson GK. Inflammation, atherosclerosis, and coronary artery disease. N Engl J Med. 2005; 352:1685-1695.

7. Stehouwer CD, Gall MA, Twisk JW, Knudsen E, Emeis JJ, Parving HH. Increased urinary albumin excretion, endothelial dysfunction, and chronic low-grade inflammation in type 2 diabetes: progressive, interrelated, and independently associated with risk of death. Diabetes. 2002; 51: 1157-1165.

8. Lichtenstein AH, Appel $\mathrm{L}$, Brands $M$, Carnethon M, Daniels S, Franch HA, Franklin B, Kris-Etherton P, Harris WS, Howard B, Karanja N, Lefevre M, Rudel L, Sacks F, Van Horn L, Winston M, Wylie-Rosett J. Diet and lifestyle recommendations revision 2006: a scientific statement from the American Heart Association Nutrition Committee. Circulation. 2006; 114:82-96.

9. Harriss LR, English DR, Powles J, Giles GG, Tonkin AM, Hodge AM, Brazionis L, O'Dea K. Dietary patterns and cardiovascular mortality in the Melbourne Collaborative Cohort Study. Am J Clin Nutr. 2007; 86:221-229.

10. Hu FB, Rimm EB, Stampfer MJ, Ascherio A, Spiegelman D, Willett WC. Prospective study of major dietary patterns and risk of coronary heart disease in men. Am J Clin Nutr. 2000; 72:912-921.

11. Knoops KT, de Groot LC, Kromhout D, Perrin AE, Moreiras-Varela O, Menotti A, van Staveren WA. Mediterranean diet, lifestyle factors, and 10-year mortality in elderly European men and women: the HALE project. Jama. 2004; 292:1433-1439.

12. Mitrou PN, Kipnis V, Thiebaut AC, Reedy J, Subar AF, Wirfalt E, Flood A, Mouw T, Hollenbeck AR, Leitzmann MF, Schatzkin A. Mediterranean dietary pattern and prediction of all-cause mortality in a US population: results from the NIH-AARP Diet and Health Study. Arch Intern Med. 2007; 167: 2461-2468.

13. Panagiotakos D, Pitsavos C, Chrysohoou C, Palliou K, Lentzas I, Skoumas I, Stefanadis C. Dietary patterns and 5-year incidence of cardiovascular disease: a multivariate analysis of the ATTICA study. Nutr Metab Cardiovasc Dis. 2009; 19:253-263.

14. Hodge AM, English DR, Itsiopoulos C, O'Dea K, Giles GG. Does a Mediterranean diet reduce the mortality risk associated with diabetes: evidence from the Melbourne Collaborative Cohort Study. Nutr Metab Cardiovasc Dis. 2011; 21:733-739.

15. Blankenberg S, Rupprecht HJ, Bickel C, Peetz D, Hafner G, Tiret L, Meyer J. Circulating cell adhesion molecules and death in patients with coronary artery disease. Circulation. 2001; 104:1336-1342.

16. de Jager J, Dekker JM, Kooy A, Kostense PJ, Nijpels G, Heine RJ, Bouter LM, Stehouwer CD. Endothelial dysfunction and low-grade inflammation explain much of the excess cardiovascular mortality in individuals with type 2 diabetes: the Hoorn Study. Arterioscler Thromb Vasc Biol. 2006; 26:1086-1093.

17. Jager A, van Hinsbergh VW, Kostense PJ, Emeis JJ, Yudkin JS, Nijpels G, Dekker JM, Heine RJ, Bouter LM, Stehouwer CD. von Willebrand factor, C-reactive protein, and 5-year mortality in diabetic and nondiabetic subjects: the Hoorn Study. Arterioscler Thromb Vasc Biol. 1999; 19:3071-3078.

18. Morange PE, Simon C, Alessi MC, Luc G, Arveiler D, Ferrieres J, Amouyel P, Evans A, Ducimetiere P, Juhan-Vague I. Endothelial cell markers and the risk of coronary heart disease: the Prospective Epidemiological Study of Myocardial Infarction (PRIME) study. Circulation. 2004; 109:1343-1348.

19. Ridker PM, Hennekens $\mathrm{CH}$, Buring JE, Rifai N. C-reactive protein and other markers of inflammation in the prediction of cardiovascular disease in women. N Engl J Med. 2000; 342:836-843.

20. Becker A, van Hinsbergh VW, Jager A, Kostense PJ, Dekker JM, Nijpels G, Heine RJ, Bouter LM, Stehouwer $\mathrm{CD}$. Why is soluble intercellular adhesion molecule-1 related to cardiovascular mortality? Eur J Clin Invest. 2002; 32:1-8. 
21. Frankel DS, Meigs JB, Massaro JM, Wilson PW, O'Donnell CJ, D'Agostino RB, Tofler GH. Von Willebrand factor, type 2 diabetes mellitus, and risk of cardiovascular disease: the framingham offspring study. Circulation. 2008; 118:2533-2539.

22. Jenny NS, Arnold AM, Kuller LH, Sharrett AR, Fried LP, Psaty BM, Tracy RP. Soluble intracellular adhesion molecule-1 is associated with cardiovascular disease risk and mortality in older adults. J Thromb Haemost. 2006; 4:107-113.

23. Luc G, Arveiler D, Evans A, Amouyel P, Ferrieres J, Bard JM, Elkhalil L, Fruchart JC, Ducimetiere P. Circulating soluble adhesion molecules ICAM-1 and VCAM-1 and incident coronary heart disease: the PRIME Study. Atherosclerosis. 2003; 170:169-176.

24. Tzoulaki I, Murray GD, Lee AJ, Rumley A, Lowe GD, Fowkes FG. Relative value of inflammatory, hemostatic, and rheological factors for incident myocardial infarction and stroke: the Edinburgh Artery Study. Circulation. 2007; 115:2119-2127.

25. Ridker PM, Hennekens CH, Roitman-Johnson B, Stampfer MJ, Allen J. Plasma concentration of soluble intercellular adhesion molecule 1 and risks of future myocardial infarction in apparently healthy men. Lancet. 1998; 351:88-92.

26. Cao JJ, Arnold AM, Manolio TA, Polak JF, Psaty BM, Hirsch CH, Kuller LH, Cushman M. Association of carotid artery intima-media thickness, plaques, and C-reactive protein with future cardiovascular disease and all-cause mortality: the Cardiovascular Health Study. Circulation. 2007; 116:32-38.

27. Eldrup N, Kragelund C, Steffensen R, Nordestgaard BG. Prognosis by C-reactive protein and matrix metalloproteinase- 9 levels in stable coronary heart disease during 15 years of follow-up. Nutr Metab Cardiovasc Dis. 2012; 22:677-683

28. Kaplan RC, McGinn AP, Baird AE, Hendrix SL, Kooperberg C, Lynch J, Rosenbaum DM, Johnson KC, Strickler HD, Wassertheil-Smoller S. Inflammation and hemostasis biomarkers for predicting stroke in postmenopausal women: the Women's Health Initiative Observational Study. J Stroke Cerebrovasc Dis. 2008; 17:344-355.

29. Ridker PM, Rifai N, Stampfer MJ, Hennekens CH. Plasma concentration of interleukin- 6 and the risk of future myocardial infarction among apparently healthy men. Circulation. 2000; 101:1767-1772.

30. Ridker PM, Rifai N, Pfeffer M, Sacks F, Lepage S, Braunwald E. Elevation of tumor necrosis factor-alpha and increased risk of recurrent coronary events after myocardial infarction. Circulation. 2000; 101:2149-2153.

31. Laurent S, Boutouyrie P, Asmar R, Gautier I, Laloux B, Guize L, Ducimetiere P, Benetos A. Aortic stiffness is an independent predictor of all-cause and cardiovascular mortality in hypertensive patients. Hypertension. 2001; 37:1236-1241.

32. Franklin SS, Khan SA, Wong ND, Larson MG, Levy D. Is pulse pressure useful in predicting risk for coronary heart Disease? The Framingham heart study. Circulation. 1999; 100:354-360.

33. Vlachopoulos C, Aznaouridis K, Stefanadis C. Prediction of cardiovascular events and all-cause mortality with arterial stiffness: a systematic review and meta-analysis. J Am Coll Cardiol. 2010; 55:1318-1327.

34. Safar ME, O'Rourke MF. Handbook of Hypertension: Arterial Stiffness in Hypertension. Vol 23. Amsterdam, Netherlands: Elsevier B.V.; 2006.

35. Stehouwer CD, Henry RM, Dekker JM, Nijpels G, Heine RJ, Bouter LM. Microalbuminuria is associated with impaired brachial artery, flow-mediated vasodilation in elderly individuals without and with diabetes: further evidence for a link between microalbuminuria and endothelial dysfunction--the Hoorn Study. Kidney Int Suppl. 2004; S42-44.

36. Brunner H, Cockcroft JR, Deanfield J, Donald A, Ferrannini E, Halcox J, Kiowski W, Luscher TF, Mancia G, Natali A, Oliver JJ, Pessina AC, Rizzoni D, Rossi GP, Salvetti A, Spieker LE, Taddei S, Webb DJ. Endothelial function and dysfunction. Part II: Association with cardiovascular risk factors and diseases. A statement by the Working Group on Endothelins and Endothelial Factors of the European Society of Hypertension. J Hypertens. 2005; 23:233-246.

37. Deanfield J, Donald A, Ferri C, Giannattasio C, Halcox J, Halligan S, Lerman A, Mancia G, Oliver JJ, Pessina AC, Rizzoni D, Rossi GP, Salvetti A, Schiffrin EL, Taddei S, Webb DJ. Endothelial function and dysfunction. Part I: Methodological issues for assessment in the different vascular beds: a statement by the Working Group on Endothelin and Endothelial Factors of the European Society of Hypertension. J Hypertens. 2005; 23:7-17. 
38. Boger $\mathrm{RH}$. The emerging role of asymmetric dimethylarginine as a novel cardiovascular risk factor. Cardiovasc Res. 2003; 59:824-833.

39. de Valk-de Roo GW, Stehouwer CD, Lambert J, Schalkwijk CG, van der Mooren MJ, Kluft C, Netelenbos C. Plasma homocysteine is weakly correlated with plasma endothelin and von Willebrand factor but not with endothelium-dependent vasodilatation in healthy postmenopausal women. Clin Chem. 1999; 45:1200-1205.

40. Devaraj S, Xu DY, Jialal I. C-reactive protein increases plasminogen activator inhibitor-1 expression and activity in human aortic endothelial cells: implications for the metabolic syndrome and atherothrombosis. Circulation. 2003; 107:398-404.

41. Eaton $C B$, Gramling R, Parker DR, Roberts MB, Lu B, Ridker PM. Prospective association of vascular endothelial growth factor-A (VEGF-A) with coronary heart disease mortality in Southeastern New England. Atherosclerosis. 2008;200:221-227.

42. Hill JM, Zalos G, Halcox JP, Schenke WH, Waclawiw MA, Quyyumi AA, Finkel T. Circulating endothelial progenitor cells, vascular function, and cardiovascular risk. N Engl J Med. 2003; 348:593-600.

43. Lekakis J, Abraham P, Balbarini A, Blann A, Boulanger CM, Cockcroft J, Cosentino F, Deanfield J, Gallino A, Ikonomidis I, Kremastinos D, Landmesser U, Protogerou A, Stefanadis C, Tousoulis D, Vassalli G, Vink H, Werner N, Wilkinson I, Vlachopoulos C. Methods for evaluating endothelial function: a position statement from the European Society of Cardiology Working Group on Peripheral Circulation. Eur J Cardiovasc Prev Rehabil. 2011;18:775-789.

44. Serne EH, Stehouwer CD, ter Maaten JC, ter Wee PM, Rauwerda JA, Donker AJ, Gans RO. Microvascular function relates to insulin sensitivity and blood pressure in normal subjects. Circulation. 1999; 99:896-902.

45. Deanfield JE, Halcox JP, Rabelink TJ. Endothelial function and dysfunction: testing and clinical relevance. Circulation. 2007; 115:1285-1295.

46. Thijssen DH, Black MA, Pyke KE, Padilla J, Atkinson G, Harris RA, Parker BA, Widlansky ME, Tschakovsky ME, Green DJ. Assessment of flow mediated dilation (FMD) in humans: a methodological and technical guideline. Am J Physiol Heart Circ Physiol. 2011; 300:H2-12.

47. Green DJ, Jones H, Thijssen D, Cable NT, Atkinson G. Flow-mediated dilation and cardiovascular event prediction: does nitric oxide matter? Hypertension. 2011; 57:363-369.

48. Joannides R, Haefeli WE, Linder L, Richard V, Bakkali EH, Thuillez C, Luscher TF. Nitric oxide is responsible for flow-dependent dilatation of human peripheral conduit arteries in vivo. Circulation. 1995; 91:1314-1319.

49. Corretti MC, Anderson TJ, Benjamin EJ, Celermajer D, Charbonneau F, Creager MA, Deanfield J, Drexler H, Gerhard-Herman M, Herrington D, Vallance P, Vita J, Vogel R. Guidelines for the ultrasound assessment of endothelial-dependent flow-mediated vasodilation of the brachial artery: a report of the International Brachial Artery Reactivity Task Force. J Am Coll Cardiol. 2002; 39:257-265.

50. Parker BA, Trehearn TL, Meendering JR. Pick your Poiseuille: normalizing the shear stimulus in studies of flow-mediated dilation. J Appl Physiol. 2009; 107:1357-1359.

51. Inaba Y, Chen JA, Bergmann SR. Prediction of future cardiovascular outcomes by flow-mediated vasodilatation of brachial artery: a meta-analysis. Int J Cardiovasc Imaging. 2010; 26:631-640.

52. Yeboah J, Crouse JR, Hsu FC, Burke GL, Herrington DM. Brachial flow-mediated dilation predicts incident cardiovascular events in older adults: the Cardiovascular Health Study. Circulation. 2007; 115:2390-2397.

53. Schram MT, Stehouwer CD. Endothelial dysfunction, cellular adhesion molecules and the metabolic syndrome. Horm Metab Res. 2005; 37 Suppl 1:49-55.

54. Yasmin, McEniery CM, Wallace S, Dakham Z, Pulsalkar P, Maki-Petaja K, Ashby MJ, Cockcroft JR, Wilkinson IB. Matrix metalloproteinase-9 (MMP-9), MMP-2, and serum elastase activity are associated with systolic hypertension and arterial stiffness. Arterioscler Thromb Vasc Biol. 2005; 25:372.

55. Ingelsson E, Hulthe J, Lind L. Inflammatory markers in relation to insulin resistance and the metabolic syndrome. Eur J Clin Invest. 2008; 38:502-509.

56. Tedgui A, Mallat Z. Cytokines in atherosclerosis: pathogenic and regulatory pathways. Physiol Rev. 2006; 86:515-581. 
57. Schnabel RB, Larson MG, Yamamoto JF, Kathiresan S, Rong J, Levy D, Keaney JF, Jr., Wang TJ, Vasan RS, Benjamin EJ. Relation of multiple inflammatory biomarkers to incident atrial fibrillation. Am J Cardiol. 2009; 104:92-96.

58. Kershaw EE, Flier JS. Adipose tissue as an endocrine organ. J Clin Endocrinol Metab. 2004; 89: 2548-2556.

59. Nin JW, Ferreira I, Schalkwijk CG, Prins MH, Chaturvedi N, Fuller JH, Stehouwer CD. Levels of soluble receptor for AGE are cross-sectionally associated with cardiovascular disease in type 1 diabetes, and this association is partially mediated by endothelial and renal dysfunction and by low-grade inflammation: the EURODIAB Prospective Complications Study. Diabetologia. 2009; 52:705-714.

60. Bantle JP, Wylie-Rosett J, Albright AL, Apovian CM, Clark NG, Franz MJ, Hoogwerf BJ, Lichtenstein AH, Mayer-Davis E, Mooradian AD, Wheeler ML. Nutrition recommendations and interventions for diabetes: a position statement of the American Diabetes Association. Diabetes Care. 2008; 31 Suppl 1:S61-78.

61. Nettleton JA, Steffen LM, Mayer-Davis EJ, Jenny NS, Jiang R, Herrington DM, Jacobs DR, Jr. Dietary patterns are associated with biochemical markers of inflammation and endothelial activation in the Multi-Ethnic Study of Atherosclerosis (MESA). Am J Clin Nutr. 2006; 83:1369-1379.

62. Lopez-Garcia E, Schulze MB, Fung TT, Meigs JB, Rifai N, Manson JE, Hu FB. Major dietary patterns are related to plasma concentrations of markers of inflammation and endothelial dysfunction. Am J Clin Nutr. 2004; 80:1029-1035.

63. Fung TT, McCullough ML, Newby PK, Manson JE, Meigs JB, Rifai N, Willett WC, Hu FB. Diet-quality scores and plasma concentrations of markers of inflammation and endothelial dysfunction. Am J Clin Nutr. 2005; 82:163-173.

64. He K, Liu K, Daviglus ML, Jenny NS, Mayer-Davis E, Jiang R, Steffen L, Siscovick D, Tsai M, Herrington D. Associations of dietary long-chain n-3 polyunsaturated fatty acids and fish with biomarkers of inflammation and endothelial activation (from the Multi-Ethnic Study of Atherosclerosis [MESA]). Am J Cardiol. 2009; 103:1238-1243.

65. Gao X, Bermudez OI, Tucker KL. Plasma C-reactive protein and homocysteine concentrations are related to frequent fruit and vegetable intake in Hispanic and non-Hispanic white elders. J Nutr. 2004; 134:913-918.

66. Wannamethee SG, Lowe GD, Rumley A, Bruckdorfer KR, Whincup PH. Associations of vitamin C status, fruit and vegetable intakes, and markers of inflammation and hemostasis. Am J Clin Nutr. 2006; 83:567-574; quiz 726-567.

67. Calder PC, Ahluwalia N, Brouns F, Buetler T, Clement K, Cunningham K, Esposito K, Jonsson LS, Kolb H, Lansink M, Marcos A, Margioris A, Matusheski N, Nordmann H, O'Brien J, Pugliese G, Rizkalla S, Schalkwijk C, Tuomilehto J, Warnberg J, Watzl B, Winklhofer-Roob BM. Dietary factors and low-grade inflammation in relation to overweight and obesity. Br J Nutr. 2011; 106 Suppl 3:S5-78.

68. Esmaillzadeh A, Azadbakht L. Home use of vegetable oils, markers of systemic inflammation, and endothelial dysfunction among women. Am J Clin Nutr. 2008; 88:913-921.

69. Song Y, Li TY, van Dam RM, Manson JE, Hu FB. Magnesium intake and plasma concentrations of markers of systemic inflammation and endothelial dysfunction in women. Am J Clin Nutr. 2007; 85:1068-1074.

70. Lopez-Garcia E, Schulze MB, Meigs JB, Manson JE, Rifai N, Stampfer MJ, Willett WC, Hu FB. Consumption of trans fatty acids is related to plasma biomarkers of inflammation and endothelial dysfunction. J Nutr. 2005; 135:562-566.

71. Shai I, Rimm EB, Schulze MB, Rifai N, Stampfer MJ, Hu FB. Moderate alcohol intake and markers of inflammation and endothelial dysfunction among diabetic men. Diabetologia. 2004; 47:1760-1767.

72. Nagel G, Zoller D, Ruf T, Rohrmann S, Linseisen J. Long-term reproducibility of a food-frequency questionnaire and dietary changes in the European Prospective Investigation into Cancer and Nutrition (EPIC)-Heidelberg cohort. Br J Nutr. 2007; 98:194-200.

73. Jacobs DR, Jr., Tapsell LC. Food, not nutrients, is the fundamental unit in nutrition. Nutr Rev. 2007; 65:439-450.

74. De Caterina R. n-3 fatty acids in cardiovascular disease. N Engl J Med. 2011; 364:2439-2450.

75. Giugliano D, Ceriello A, Esposito K. The effects of diet on inflammation: emphasis on the metabolic syndrome. J Am Coll Cardiol. 2006; 48:677-685. 
76. Brown AA, Hu FB. Dietary modulation of endothelial function: implications for cardiovascular disease. Am J Clin Nutr. 2001; 73:673-686.

77. De Caterina R, Zampolli A, Del Turco S, Madonna R, Massaro M. Nutritional mechanisms that influence cardiovascular disease. Am J Clin Nutr. 2006; 83:421S-426S.

78. Davis N, Katz S, Wylie-Rosett J. The effect of diet on endothelial function. Cardiol Rev. 2007; 15:62-66.

79. Galland L. Diet and inflammation. Nutr Clin Pract. 2010; 25:634-640.

80. Hall WL. Dietary saturated and unsaturated fats as determinants of blood pressure and vascular function. Nutr Res Rev. 2009; 22:18-38.

81. Corti R, Flammer AJ, Hollenberg NK, Luscher TF. Cocoa and cardiovascular health. Circulation. 2009; 119:1433-1441.

82. Bau PF, Bau CH, Rosito GA, Manfroi WC, Fuchs FD. Alcohol consumption, cardiovascular health, and endothelial function markers. Alcohol. 2007; 41:479-488.

83. Gresele P, Cerletti C, Guglielmini G, Pignatelli P, de Gaetano G, Violi F. Effects of resveratrol and other wine polyphenols on vascular function: an update. J Nutr Biochem. 2011; 22:201-211.

84. Schini-Kerth VB, Auger C, Kim JH, Etienne-Selloum N, Chataigneau T. Nutritional improvement of the endothelial control of vascular tone by polyphenols: role of NO and EDHF. Pflugers Arch. 2010; 459:853-862.

85. Willett WC. Nutritional Epidemiology. 2th ed. New York, NY: Oxford University Press; 1998.

86. Thompson FE, Subar AF. Dietary assessment methodology. In: Coulston AM, Boushey CJ, eds. Nutrition in the prevention and treatment of disease. 2nd ed. San Diego, CA: Academic Press; 2008.

87. Burke BS. The dietary history as a tool in research. J Am Diet Assoc. 1947; 1041-1046.

88. Illner AK, Nothlings $\mathrm{U}$, Wagner $\mathrm{K}$, Ward H, Boeing $\mathrm{H}$. The assessment of individual usual food intake in large-scale prospective studies. Ann Nutr Metab. 2010; 56:99-105.

89. Cade J, Thompson R, Burley V, Warm D. Development, validation and utilisation of food-frequency questionnaires - a review. Public Health Nutr. 2002; 5:567-587.

90. Ocke MC, Bueno-de-Mesquita HB, Goddijn HE, Jansen A, Pols MA, van Staveren WA, Kromhout D. The Dutch EPIC food frequency questionnaire. I. Description of the questionnaire, and relative validity and reproducibility for food groups. Int J Epidemiol. 1997; 26 Suppl 1:S37-48.

91. Ocke MC, Bueno-de-Mesquita HB, Pols MA, Smit HA, van Staveren WA, Kromhout D. The Dutch EPIC food frequency questionnaire. II. Relative validity and reproducibility for nutrients. Int J Epidemiol. 1997; 26 Suppl 1:S49-58.

92. Toeller M, Klischan A, Heitkamp G, Schumacher W, Milne R, Buyken A, Karamanos B, Gries FA. Nutritional intake of 2868 IDDM patients from 30 centres in Europe. EURODIAB IDDM Complications Study Group. Diabetologia. 1996; 39:929-939.

93. Toeller M, Buyken A, Heitkamp G, Milne R, Klischan A, Gries FA. Repeatability of three-day dietary records in the EURODIAB IDDM Complications Study. Eur J Clin Nutr. 1997; 51:74-80.

94. McEniery CM, Qasem A, Schmitt M, Avolio AP, Cockcroft JR, Wilkinson IB. Endothelin-1 regulates arterial pulse wave velocity in vivo. J Am Coll Cardiol. 2003; 42:1975-1981.

95. Wilkinson IB, Qasem A, McEniery CM, Webb DJ, Avolio AP, Cockcroft JR. Nitric oxide regulates local arterial distensibility in vivo. Circulation. 2002; 105:213-217.

96. Greenwald SE. Ageing of the conduit arteries. J Pathol. 2007; 211:157-172.

97. Stehouwer CD, Henry RM, Ferreira I. Arterial stiffness in diabetes and the metabolic syndrome: a pathway to cardiovascular disease. Diabetologia. 2008; 51:527-539.

98. Laurent S, Cockcroft J, Van Bortel L, Boutouyrie P, Giannattasio C, Hayoz D, Pannier B, Vlachopoulos C, Wilkinson I, Struijker-Boudier H. Expert consensus document on arterial stiffness: methodological issues and clinical applications. Eur Heart J. 2006; 27:2588-2605.

99. Abramson JL, Weintraub WS, Vaccarino V. Association between pulse pressure and C-reactive protein among apparently healthy US adults. Hypertension. 2002; 39:197-202.

100. Amar J, Ruidavets JB, Bal Dit Sollier C, Bongard V, Boccalon H, Chamontin B, Drouet L, Ferrieres J. Soluble CD14 and aortic stiffness in a population-based study. J Hypertens. 2003; 21:1869-1877.

101. Amar J, Ruidavets JB, Sollier CB, Bongard V, Boccalon H, Chamontin B, Drouet L, Ferrieres J. Relationship between $C$ reactive protein and pulse pressure is not mediated by atherosclerosis or aortic stiffness. J Hypertens. 2004; 22:349-355.

102. Chae CU, Lee RT, Rifai N, Ridker PM. Blood pressure and inflammation in apparently healthy men. Hypertension. 2001; 38:399-403. 
103. Eklund C, Kivimaki M, Islam MS, Juonala M, Kahonen M, Marniemi J, Lehtimaki T, Viikari J, Raitakari OT, Hurme M. C-reactive protein genetics is associated with carotid artery compliance in men in The Cardiovascular Risk in Young Finns Study. Atherosclerosis. 2008; 196:841-848.

104. Juonala M, Jarvisalo MJ, Maki-Torkko N, Kahonen M, Viikari JS, Raitakari OT. Risk factors identified in childhood and decreased carotid artery elasticity in adulthood: the Cardiovascular Risk in Young Finns Study. Circulation. 2005; 112:1486-1493.

105. Kampus P, Kals J, Ristimae T, Fischer K, Zilmer M, Teesalu R. High-sensitivity C-reactive protein affects central haemodynamics and augmentation index in apparently healthy persons. J Hypertens. 2004; 22:1133-1139.

106. Kampus P, Kals J, Ristimae T, Muda P, Ulst K, Zilmer K, Salonen RM, Tuomainen TP, Teesalu R, Zilmer $M$. Augmentation index and carotid intima-media thickness are differently related to age, $C$-reactive protein and oxidized low-density lipoprotein. J Hypertens. 2007; 25:819-825.

107. Kullo IJ, Seward JB, Bailey KR, Bielak LF, Grossardt BR, Sheedy PF 2nd, Peyser PA, Turner ST. C-reactive protein is related to arterial wave reflection and stiffness in asymptomatic subjects from the community. Am J Hypertens. 2005; 18:1123-1129.

108. Lieb W, Larson MG, Benjamin EJ, Yin X, Tofler GH, Selhub J, Jacques PF, Wang TJ, Vita JA, Levy D, Vasan RS, Mitchell GF. Multimarker approach to evaluate correlates of vascular stiffness: the Framingham Heart Study. Circulation. 2009; 119:37-43.

109. Mattace-Raso FU, van der Cammen TJ, van der Meer IM, Schalekamp MA, Asmar R, Hofman A, Witteman JC. C-reactive protein and arterial stiffness in older adults: the Rotterdam Study. Atherosclerosis. 2004; 176:111-116.

110. Nakhai-Pour HR, Grobbee DE, Bots ML, Muller M, van der Schouw YT. C-reactive protein and aortic stiffness and wave reflection in middle-aged and elderly men from the community. J Hum Hypertens. 2007; 21:949-955.

111. Schnabel R, Larson MG, Dupuis J, Lunetta KL, Lipinska I, Meigs JB, Yin X, Rong J, Vita JA, Newton-Cheh C, Levy D, Keaney JF, Jr., Vasan RS, Mitchell GF, Benjamin EJ. Relations of inflammatory biomarkers and common genetic variants with arterial stiffness and wave reflection. Hypertension. 2008; 51:1651-1657.

112. Schumacher W, Cockcroft J, Timpson NJ, McEniery CM, Gallacher J, Rumley A, Lowe G, Smith GD, Wilkinson IB, Ben-Shlomo Y. Association between C-reactive protein genotype, circulating levels, and aortic pulse wave velocity. Hypertension. 2009; 53:150-157.

113. Schutte R, Schutte AE, Van Rooyen JM, Huisman HW, Palmer IM, Fourie CM, Peter S, Malan L, Malan NT, Reimann M. Von Willebrand factor as marker of vascular function in South African women: the POWIRS Study. Am J Hypertens. 2008; 21:1298-1303.

114. Whincup PH, Gilg JA, Donald AE, Katterhorn M, Oliver C, Cook DG, Deanfield JE. Arterial distensibility in adolescents: the influence of adiposity, the metabolic syndrome, and classic risk factors. Circulation. 2005; 112:1789-1797.

115. Yasmin, McEniery CM, Wallace S, Mackenzie IS, Cockcroft JR, Wilkinson IB. C-reactive protein is associated with arterial stiffness in apparently healthy individuals. Arterioscler Thromb Vasc Biol. 2004; 24:969-974.

116. Nichols WW, O'Rourke MF. McDonald's Blood Flow in Arteries: Theoretical, Experimental and Clinical Principles 5th ed. London, UK: Oxford University Press Inc.; 2005.

117. Henry RM, Kostense PJ, Spijkerman AM, Dekker JM, Nijpels G, Heine RJ, Kamp O, Westerhof N, Bouter LM, Stehouwer CD. Arterial stiffness increases with deteriorating glucose tolerance status: the Hoorn Study. Circulation. 2003; 107:2089-2095.

118. Van Bortel LM, Balkestein EJ, van der Heijden-Spek JJ, Vanmolkot FH, Staessen JA, Kragten JA, Vredeveld JW, Safar ME, Struijker Boudier HA, Hoeks AP. Non-invasive assessment of local arterial pulse pressure: comparison of applanation tonometry and echo-tracking. J Hypertens. 2001; 19:10371044.

119. Yang EY, Chambless L, Sharrett AR, Virani SS, Liu X, Tang Z, Boerwinkle E, Ballantyne CM, Nambi V. Carotid arterial wall characteristics are associated with incident ischemic stroke but not coronary heart disease in the Atherosclerosis Risk in Communities (ARIC) study. Stroke. 2012; 43:103-108.

120. Kemper HC. Amsterdam Growth and Health Longitudinal Study (AGAHLS): A 23-Year Follow-up From Teenage to Adult About Lifestyle and Health. Basel, Switzerland: Karger; 2004. 
121. Ferreira I, Henry RM, Twisk JW, van Mechelen W, Kemper HC, Stehouwer CD. The metabolic syndrome, cardiopulmonary fitness, and subcutaneous trunk fat as independent determinants of arterial stiffness: the Amsterdam Growth and Health Longitudinal Study. Arch Intern Med. 2005; 165:875-882.

122. Schouten F, Twisk JW, de Boer MR, Stehouwer CD, Serne EH, Smulders YM, Ferreira I. Increases in central fat mass and decreases in peripheral fat mass are associated with accelerated arterial stiffening in healthy adults: the Amsterdam Growth and Health Longitudinal Study. Am J Clin Nutr. 2011; 94:40-48.

123. Ferreira I, Twisk JW, van Mechelen W, Kemper HC, Stehouwer CD. Development of fatness, fitness, and lifestyle from adolescence to the age of 36 years: determinants of the metabolic syndrome in young adults: the amsterdam growth and health longitudinal study. Arch Intern Med. 2005; 165: 42-48.

124. de Vente W, Post GB, Twisk JW, Kemper HC, van Mechelen W. Effects of health measurements and health information in youth and young adulthood in dietary intake--20-y study results from the Amsterdam Growth and Health Longitudinal Study. Eur J Clin Nutr. 2001; 55:819-823.

125. Bakker I, Twisk JW, van Mechelen W, Mensink GB, Kemper HC. Computerization of a dietary history interview in a running cohort; evaluation within the Amsterdam Growth and Health Longitudinal Study. Eur J Clin Nutr. 2003; 57:394-404.

126. Ferreira I, Twisk JW, Van Mechelen W, Kemper HC, Stehouwer CD. Current and adolescent levels of cardiopulmonary fitness are related to large artery properties at age 36: the Amsterdam Growth and Health Longitudinal Study. Eur J Clin Invest. 2002; 32:723-731.

127. van Mechelen W, Twisk JW, Post GB, Snel J, Kemper HC. Physical activity of young people: the Amsterdam Longitudinal Growth and Health Study. Med Sci Sports Exerc. 2000; 32:1610-1616.

128. Mooy JM, Grootenhuis PA, de Vries H, Valkenburg HA, Bouter LM, Kostense PJ, Heine RJ. Prevalence and determinants of glucose intolerance in a Dutch caucasian population. The Hoorn Study. Diabetes Care. 1995; 18:1270-1273.

129. Spijkerman AM, Adriaanse MC, Dekker JM, Nijpels G, Stehouwer CD, Bouter LM, Heine RJ. Diabetic patients detected by population-based stepwise screening already have a diabetic cardiovascular risk profile. Diabetes Care. 2002; 25:1784-1789.

130. Henry RM, Ferreira I, Kostense PJ, Dekker JM, Nijpels G, Heine RJ, Kamp O, Bouter LM, Stehouwer CD. Type 2 diabetes is associated with impaired endothelium-dependent, flow-mediated dilation, but impaired glucose metabolism is not; The Hoorn Study. Atherosclerosis. 2004; 174:49-56.

131. Du $H$, van der AD, van Bakel MM, van der Kallen CJ, Blaak EE, van Greevenbroek MM, Jansen EH, Nijpels G, Stehouwer CD, Dekker JM, Feskens EJ. Glycemic index and glycemic load in relation to food and nutrient intake and metabolic risk factors in a Dutch population. Am J Clin Nutr. 2008; 87: 655-661.

132. Kruijshoop M, Feskens EJ, Blaak EE, de Bruin TW. Validation of capillary glucose measurements to detect glucose intolerance or type 2 diabetes mellitus in the general population. Clin Chim Acta. 2004; 341:33-40.

133. Jacobs $M$, van Greevenbroek MM, van der Kallen CJ, Ferreira I, Blaak EE, Feskens EJ, Jansen EH, Schalkwijk CG, Stehouwer CD. Low-grade inflammation can partly explain the association between the metabolic syndrome and either coronary artery disease or severity of peripheral arterial disease: the CODAM study. Eur J Clin Invest. 2009; 39:437-444.

134. Jacobs M, van Greevenbroek MM, van der Kallen CJ, Ferreira I, Blaak EE, Feskens EJ, Jansen EH, Schalkwijk CG, Stehouwer CD. The association between the metabolic syndrome and peripheral, but not coronary, artery disease is partly mediated by endothelial dysfunction: the CODAM study. Eur J Clin Invest. 2010; 41:167-175.

135. Thewissen MM, Damoiseaux JG, Duijvestijn AM, van Greevenbroek MM, van der Kallen CJ, Feskens EJ, Blaak EE, Schalkwijk CG, Stehouwer CD, Tervaert JW, Ferreira I. Abdominal Fat Mass Is Associated With Adaptive Immune Activation: The CODAM Study. Obesity (Silver Spring). 2010; 19:1690-1698.

136. van Greevenbroek MM, Jacobs M, van der Kallen CJ, Blaak EE, Jansen EH, Schalkwijk CG, Feskens EJ, Stehouwer CD. Human plasma complement C3 is independently associated with coronary heart disease, but only in heavy smokers (the CODAM study). Int J Cardiol. 2012; 154:158-162.

137. Chaturvedi N, Bandinelli S, Mangili R, Penno G, Rottiers RE, Fuller JH. Microalbuminuria in type 1 diabetes: rates, risk factors and glycemic threshold. Kidney Int. 2001; 60:219-227. 
138. Microvascular and acute complications in IDDM patients: the EURODIAB IDDM Complications Study. Diabetologia. 1994; 37:278-285.

139. Chaturvedi N, Fuller JH, Taskinen MR. Differing associations of lipid and lipoprotein disturbances with the macrovascular and microvascular complications of type 1 diabetes. Diabetes Care. 2001; 24: 2071-2077.

140. Schram MT, Chaturvedi N, Schalkwijk C, Giorgino F, Ebeling P, Fuller JH, Stehouwer CD. Vascular risk factors and markers of endothelial function as determinants of inflammatory markers in type 1 diabetes: the EURODIAB Prospective Complications Study. Diabetes Care. 2003; 26:2165-2173.

141. Toeller M, Buyken A, Heitkamp G, Bramswig S, Mann J, Milne R, Gries FA, Keen H. Protein intake and urinary albumin excretion rates in the EURODIAB IDDM Complications Study. Diabetologia. 1997; 40:1219-1226.

142. Buyken AE, Toeller M, Heitkamp G, Vitelli F, Stehle P, Scherbaum WA, Fuller JH. Relation of fibre intake to $\mathrm{HbA} 1 \mathrm{c}$ and the prevalence of severe ketoacidosis and severe hypoglycaemia. EURODIAB IDDM Complications Study Group. Diabetologia. 1998; 41:882-890. 
Part I

Methodology of biomarker detection 


\section{Chapter 2}

Multiple inflammatory biomarker detection in a prospective cohort study: a cross-validation between well-established single-biomarker techniques and an electrochemiluminescence-based multi-array platform

BCT van Bussel, I Ferreira, MPH van de Waarenburg, MMJ van Greevenbroek, CJH van der Kallen, RMA Henry, EJM Feskens, CDA Stehouwer, CG Schalkwijk

PLoS One. 2013;8:e58576

Reprinted with permission of the publisher 


\section{Abstract}

\section{Background}

In terms of time, effort and quality, multiplex technology is an attractive alternative for wellestablished single-biomarker measurements in clinical studies. However, limited data comparing these methods are available.

\section{Methods}

We measured, in a large ongoing cohort study ( $n=574)$, by means of both a 4-plex multi-array biomarker assay developed by MesoScaleDiscovery (MSD) and single-biomarker techniques (ELISA or immunoturbidimetric assay), the following biomarkers of low-grade inflammation: Creactive protein (CRP), serum amyloid $A(S A A)$, soluble intercellular adhesion molecule 1 (sICAM-1) and soluble vascular cell adhesion molecule 1 (sVCAM-1). These measures were realigned by weighted Deming regression and compared across a wide spectrum of subjects' cardiovascular risk factors by ANOVA.

\section{Results}

Despite that both methods ranked individuals' levels of biomarkers very similarly (Pearson's $r$ all $\geq 0.755$ ) absolute concentrations of all biomarkers differed significantly between methods. Equations retrieved by Deming regression enabled proper realignment of the data to overcome these differences, such that intra-class correlation coefficients were then 0.996 (CRP), 0.711 (SAA), 0.895 (sICAM-1) and 0.858 (sVCAM-1). Additionally, individual biomarkers differed across categories of glucose metabolism, weight, metabolic syndrome and smoking status to a similar extent by either method.

\section{Conclusions}

Multiple low-grade inflammatory biomarker data obtained by the 4-plex multi-array platform of MSD or by well-established single-biomarker methods are comparable after proper realignment of differences in absolute concentrations, and are equally associated with cardiovascular risk factors, regardless of such differences. Given its greater efficiency, the MSD platform is a potential tool for the quantification of multiple biomarkers of low-grade inflammation in large ongoing and future clinical studies. 


\section{Introduction}

Biomarker measurements representing low-grade inflammation have gained increasing importance in the management and understanding of cardiovascular disease (CVD). ${ }^{1-8}$ Low-grade inflammation is accompanied by inflammatory cells that closely interact with the arterial wall, thereby driving the development of atherosclerosis and CVD. ${ }^{1,2}$ Because the role of low-grade inflammation in the pathophysiology of CVD is multi-faceted ${ }^{1,2}$, an extensive characterization by multiple biomarkers of low-grade inflammation is desirable. In this line, large cohort studies are increasingly adopting such a multiple biomarker approach. ${ }^{8-15}$

Among a large variety of potential biomarkers ${ }^{16}$, acute-phase reactants such as C-reactive protein (CRP) and serum amyloid A (SAA), and vascular adhesion molecules such as soluble intercellular adhesion molecule-1 (sICAM-1) and soluble vascular cell adhesion molecule-1 (sVCAM-1) reflect low-grade inflammation when present in low concentrations $^{1,2}$, whereas high concentrations, such as CRP $>10 \mathrm{mg} / \mathrm{l}$, more likely reflect acute inflammation or infection., ${ }^{9,17,18}$ These four biomarkers of low-grade inflammation were studied because they play an important role in the pathophysiology of $\mathrm{CVD}^{1,2}$ and higher concentrations have been associated with (incident) CVD.

Traditionally, well-established analytical methods have enabled the analysis of single biomarkers of low-grade inflammation in one run. However, obtaining multiple biomarkers based on many single-biomarker measurements is very labour intensive, expensive and requires (relatively) large sample volumes. These limitations hamper an efficient multiple biomarker approach, particularly in large observational cohort or clinical trial studies. An attractive solution to these limitations is the simultaneous, and thus more efficient, measurement of a set of low-grade inflammatory biomarkers in one run. Such methods have recently become available with the use of multi-array platforms, such as the Luminex ${ }^{\circledast}$ and the MesoScaleDiscovery ${ }^{\circledast}$ (MSD) platforms and provide the tools necessary for efficient multiple biomarker detection. However, it remains to be established to what extent biomarker concentrations, as measured with these multi-array platforms, are comparable to well-established single-biomarker measurements. Although some cross-validation studies have been performed, most have not focused on biomarkers of low-grade inflammation ${ }^{19-23}$ and the only study that did so pointed the problem of different measured concentrations, which may lead to bias in epidemiological associations. ${ }^{23}$

Therefore, introducing a multi-array platform in the context of an ongoing longitudinal cohort study poses some challenges ${ }^{24,25}$ and cross-validation between methods within such a cohort is necessary before the 'new' method may replace the 'old' one. Specifically, one needs to determine whether substantial differences in biomarker concentrations are introduced by the new method, in which case realignment of the data by appropriate mathematical transformations may be required for the investigation of within-subjects changes in absolute concentrations of biomarkers 
over the course of time. ${ }^{26,27}$ In addition, the data obtained need also to be similarly associated with risk factors (RFs) known to be associated with low-grade inflammation to ensure that the multi-array platform measures what it intends to measure (i.e. face validity).

In view of these considerations, we compared the performance of a 4-plex multi-array electrochemiluminescence detection platform of low-grade inflammatory biomarkers (CRP, SAA, SICAM-1 and SVCAM-1) of MSD with that of well-established singlebiomarker measurements, in a large ongoing cohort study of individuals with a wide spectrum of cardiovascular risk factors (RFs) known to be associated with low-grade inflammation.

\section{Materials and methods}

\section{Ethics statement}

The study was approved by the Medical Ethical Committee of the Maastricht University and all individuals gave written informed consent.

\section{Study population and design}

The Cohort on Diabetes and Atherosclerosis Maastricht (CODAM) is a prospective cohort study that was originally designed to study the effects of obesity, glucose and lipid metabolism, lifestyle and genetics on cardiovascular complications, as described in detail elsewhere. ${ }^{28-33}$ Briefly, individuals were selected from a large populationbased cohort and included if they were of Caucasian ethnicity and older than 40 years, and met one or more of the following criteria: a body mass index (BMI) $\geq 25 \mathrm{~kg} / \mathrm{m}^{2}$, a positive family history for type 2 diabetes mellitus, a history of gestational diabetes, use of anti-hypertensive medication, a postprandial glucose $\geq 6.0 \mathrm{mmol} / \mathrm{l}$ and/or glucosuria. In total, 574 individuals (mean age $59.6 \pm 7.0$ years; $38.7 \%$ women) were included and extensively characterized with regard to their metabolic, cardiovascular and lifestyle risk profiles during 2 visits to the University research unit (CODAM-1, baseline examination: September 1999-July 2002). A first follow-up examination took place among 495 individuals (14\% drop-out rate, mainly due to morbidity or mortality) approximately 7 years later (CODAM-2, July 2006-November 2009).

At baseline (i.e. CODAM-1), biomarkers of low-grade inflammation were assessed by single-biomarker techniques. At follow-up (i.e. CODAM-2), the single-biomarker techniques were replaced by the multi-array platform of MSD. To ensure comparability between methods, biomarkers of low-grade inflammation were also reassayed by the multi-array platform of MSD in all samples from the baseline examination (i.e. CODAM-1); at the time of these measurements, baseline samples had thus been stored for $\sim 7$ years. The present cross-validation study reports on 
individuals' paired data on biomarkers during the baseline examination (CODAM-1) and thus is a cross-sectional method comparison study. Method comparison for each biomarker was conducted on paired data, which were available for CPR in 566 individuals, for SAA in 563 individuals, for sICAM-1 in 566 individuals and for SVCAM-1 in 567 individuals and full paired data on all four inflammatory biomarkers were available in 550 individuals.

The CODAM study population is characterized by a wide spectrum of conditions known to be associated with low-grade inflammation. ${ }^{28-33}$ Specifically, $52.4 \%$ of the individuals had normal glucose metabolism (NGM), $22.2 \%$ had impaired glucose metabolism (IGM) and $25.4 \%$ had type 2 diabetes mellitus (DM2). ${ }^{28-33}$ On the basis of measured $\mathrm{BMI}$, the prevalence of normal weight, overweight and obesity was 18.2 , 50.9 and $30.9 \%$, respectively. ${ }^{32}$ The metabolic syndrome, i.e. the clustering of $\geq 3$ out of 5 cardiovascular RFs reflecting central obesity, dyslipidaemia, elevated blood pressure and fasting plasma glucose, was prevalent in $54.7 \%$ of the study population. $^{30,31}$ On the basis of self-reports, $29.3 \%$ of the individuals were neversmokers, $50.5 \%$ were ex-smokers and $20.2 \%$ were current smokers. ${ }^{33}$ The prevalence of CVD was $27.6 \%$ and based on self-reports of myocardial infarction, coronary bypass surgery, stent placement or balloon dilatation, transient ischaemic attack or stroke, abnormalities on a 12-lead electrocardiogram (Minnesota codes 1.1 to $1.3,4.1$ to 4.3, 5.1 to 5.3 or 7.1 ) and/or self-reported narrowing of peripheral arteries, non-traumatic limb amputation or a measured ankle-arm index $<0.9 .{ }^{30,31}$ Glycated haemoglobin [(mean \pm SD) $6.0 \pm 0.82 \%]$ was measured as previously described. ${ }^{28-33}$ Estimated glomerular filtration rate (eGFR) $\left[\left(\right.\right.$ mean \pm SD) $\left.95.7 \pm 19.0 \mathrm{ml} / \mathrm{min} / 1.73 \mathrm{~m}^{2}\right]$ was calculated on the basis of individuals' age, sex and serum creatinine levels according to the short Modification of Diet in Renal Disease (MDRD) equation. ${ }^{34}$

\section{Biomarker assessments}

Individuals were asked to stop their lipid-lowering medication 14 days prior to the blood withdrawals and all other medication on the day before. After an overnight fast (duration of at least 10 hours) blood was drawn from the anticubital vein and collected in EDTA polypropylene tubes for plasma and in clot activator containing polypropylene tubes for serum. EDTA tubes were centrifuged at $3000 \mathrm{rpm}$ for 15 minutes at $4^{\circ} \mathrm{C}$, and plasma was immediately divided into $1 \mathrm{ml}$ aliquots and stored in $-80^{\circ} \mathrm{C}$ freezers until further analysis. Tubes with cloth activator were left 20 minutes before centrifugation at $3000 \mathrm{rpm}$ for 15 minutes at $20^{\circ} \mathrm{C}$, and serum was immediately divided into $1 \mathrm{ml}$ aliquots and stored in $-20^{\circ} \mathrm{C}$ freezers until analysis. ${ }^{28}$

\section{Biomarker detection by single-biomarker techniques.}

CRP was measured in a single measurement in serum with a high-sensitivity, immunoturbidimetric assay (detection range $100 \mathrm{ng} / \mathrm{ml}$ to $20000 \mathrm{ng} / \mathrm{ml}$, i.e. factor 200) (Latex, Roche Diagnostics Netherlands BV, Almere, The Netherlands, 
www.roche.nl). This assay is based on the principle of particle-enhanced immunological agglutination. Briefly, anti-CRP antibodies coupled to latex microparticles react with antigen present in the sample to form antigen-antibody complexes. Then, these micro-particles with antigen-antibody complexes agglutinate. This changes the fluid turbidity of the sample, which is detected by turbidimetry. sVCAM-1 was measured in EDTA plasma with a high-sensitivity human Quantikine ELISA kit (detection range $6.25 \mathrm{ng} / \mathrm{ml}$ to $200 \mathrm{ng} / \mathrm{ml}$, i.e. factor 32) (R\&D Systems, Minneapolis, MN, USA, www.rndsystems.com). sICAM-1 (detection range $0.625 \mathrm{ng} / \mathrm{ml}$ to $10 \mathrm{ng} / \mathrm{ml}$, i.e. factor 16 ) and SAA (detection range $9.4 \mathrm{ng} / \mathrm{ml}$ to $600 \mathrm{ng} / \mathrm{ml}$, i.e. factor 64) were measured in EDTA plasma by ELISA (Biosource, Invitrogen, Carlsbad, CA, USA, www.invitrogen.com). All low-grade inflammatory biomarkers were measured at the Laboratory of Toxicology, Genetics and Pathology of the National Institute for Public Health and the Environment, Bilthoven, The Netherlands. ${ }^{30}$ The intra- and inter-assay coefficients of variation (CVs) for these assays were, for CRP, 0.6 and $1.9 \%$; for SAA, 6.1 and $17.5 \%$; for SICAM-1, 5.6 and $6.6 \%$; and for SVCAM-1, 3.1 and $4.7 \%$, respectively.

\section{Biomarker detection by the 4-plex multi-array electrochemiluminescence detection platform of MesoScaleDiscovery}

The 4-plex multi-array electrochemiluminescence platform of MesoScaleDiscovery (detection range $0.008 \mathrm{ng} / \mathrm{ml}$ to $1000 \mathrm{ng} / \mathrm{ml}$, i.e. factor 125000) (MesoScaleDiscovery, Gaithersburg, MD, USA, www.mesoscale.com) was used to measure the four lowgrade inflammatory biomarkers (CRP, SAA, sICAM-1 and sVCAM-1) simultaneously in EDTA plasma. This system uses multi-array plates fitted with multi-electrodes per well with each electrode being coated with a different capture antibody. For the present study the 4-plex assay (plates fitted with four electrodes per well, i.e. four separate well spots with a different capture antibody bound to each) was used. The assay procedure follows that of a classic sandwich ELISA with any of the analytes of interest captured on the relevant electrode. These captured analytes were, in turn, detected by a secondary, analyte-specific, ruthenium-conjugated antibody, which is capable of emitting light after electrochemical stimulation. This method minimizes nonspecific signals as the stimulation mechanism (electricity) is decoupled from the signal (light). According to the MSD protocol, each sample was analyzed in duplicate on the same array plate. All multi-array plates were analyzed within 16 days. The intra- and interassay CVs for the platform of MSD were, for CRP, 3.0 and 4.1\%; for SAA, 2.5 and 11.8\%; for sICAM-1, 2.5 and 4.7\%; and, for SVCAM-1, 2.6 and 5.0\%, respectively.

Variation between production lots of multi-array plates could influence biomarker measurements. We have evaluated the possible effect of lot-to-lot variation in the current 4-plex assay using additional data of previous studies. ${ }^{35,36}$ Based on biomarker data of six separate production lots (with an average of 30 plates per lot) the lot-to-lot CV for CRP was $9.8 \%$, for SAA was $28.9 \%$, for sICAM-1 was $3.4 \%$ and for SVCAM-1 was $4.9 \%$. Thus, these variations were quite acceptable, except for SAA. Still, to avoid any 
noise due to lot-to-lot variation, all plasma samples of the CODAM study were measured within a single production lot of multi-array plates.

\section{Statistical analyses}

\section{Method Comparisons}

Absolute concentrations of each biomarker as measured by the single-biomarker techniques and the multi-array platform were examined on all paired samples from the CODAM Study baseline examination ( $n=566$ for CRP, $n=563$ for SAA, $n=566$ for sICAM-1 and $n=567$ for sVCAM-1, after exclusion of erroneous outliers ${ }^{37}$ ). Pearson's correlation coefficients were used to assess whether the ranking of each biomarker was similar between methods. Weighted Deming regression was used to assess the extent of constant and/or proportional bias between methods. ${ }^{26,27}$ This state-of-theart statistical technique for method comparison is superior to simple linear regression by taking into account the error in both the dependent and independent variables. ${ }^{37,38}$ In addition, it allows random errors of each method to be proportional to the measured concentrations, such that the ratio of the CVs between methods remains constant over the concentration ranges (set at 1:1 for regression calculations; e.g. $2 \%$ vs. $2 \%$ at low ranges, and $10 \%$ vs. $10 \%$ at high ranges). ${ }^{37,38}$

\section{Realignment and agreement}

We anticipated that absolute biomarker concentrations, as obtained by either singleor multi-array methods, would differ due to a lack of standardization. Realignment of the data would, therefore, be necessary to enable direct comparison of absolute concentrations. For that purpose we used equations derived from Deming regression analyses to realign the data as obtained by one to the other method.

To examine the levels of agreement and verify the absence of systematic error after the realignment procedure, Bland-Altman plots of the differences between singlebiomarker and multi-array data vs. their mean were obtained. ${ }^{39}$ Bland-Altman plots were drawn on $\log _{e}$ transformed data whenever the distribution of the differences was skewed. ${ }^{39,40}$ In addition, two-way mixed effects models (absolute agreement) were used to calculate intra-class correlation coefficients (ICC), which reflect similarity in individuals' rank and similarity in absolute biomarker concentrations as obtained by single-biomarker techniques (realigned) and multi-array platform. ${ }^{41}$ Note that the results of these analyses are shown in detail for single-biomarker data realigned to multi-array data for the following reason. The multi-array platform has recently been introduced in the CODAM study population and represents the methodology intended to carry on in follow-up assessments in this cohort. 
Method performance across different cardiovascular risk groups

We used ANOVA to investigate the extent to which biomarker concentrations, either assessed by the single-biomarker techniques or the multi-array platform, increased across categories of glucose metabolism (i.e. NGM, IGM and DM2), weight (i.e. normal weight, overweight and obesity), number of traits of the metabolic syndrome (0-1 RFs, 2 RFs and $\geq 3$ RFs) and smoking status (never, ex- and current-smoker), by appreciation of the group effects. ANOVA for repeated measures were subsequently used to ascertain whether such patterns of associations were similar between methods, by appreciation of group-by-method effects (the p-values of which should then be 20.05). In these analyses, (non-aligned) individual biomarker data, which are expressed in different scale units, were first standardized to comparable units by calculation of Z-scores as follows: (the individuals' value - the population mean) \the population SD. Per definition, each Z-score has a mean of 0 , a SD of 1 , and the same distribution as the absolute biomarker concentration (i.e. the ranking of individuals in the population remains the same). This thus enabled a direct comparison of the magnitude of relative differences in each biomarker by RF categories. All comparisons included adjustments for sex, age, eGFR and prior CVD and were conducted among individuals with complete paired data on all four biomarkers $(n=550)$.

All analyses were performed with the use of the Statistical Package for Social Sciences (SPSS Inc, version 15.0, Chicago, Illinois, USA, www.spss.com), except weighted Deming regression, which was analyzed using the Analyse-lt software (Analyse-it Software Ltd, Leeds, UK, www.analyse-it.com) for Microsoft Excel (Microsoft Corporation, Washington, USA, www.microsoft.com). Statistical significance was set at a $\mathrm{p}$-value $<0.05$.

\section{Results}

\section{Biomarker concentrations}

Table 2.1 shows the absolute concentrations of CRP, SAA, sICAM-1, and SVCAM-1, as measured with the single-biomarker techniques or the multi-array platform, in the whole study population and across RFs categories. 


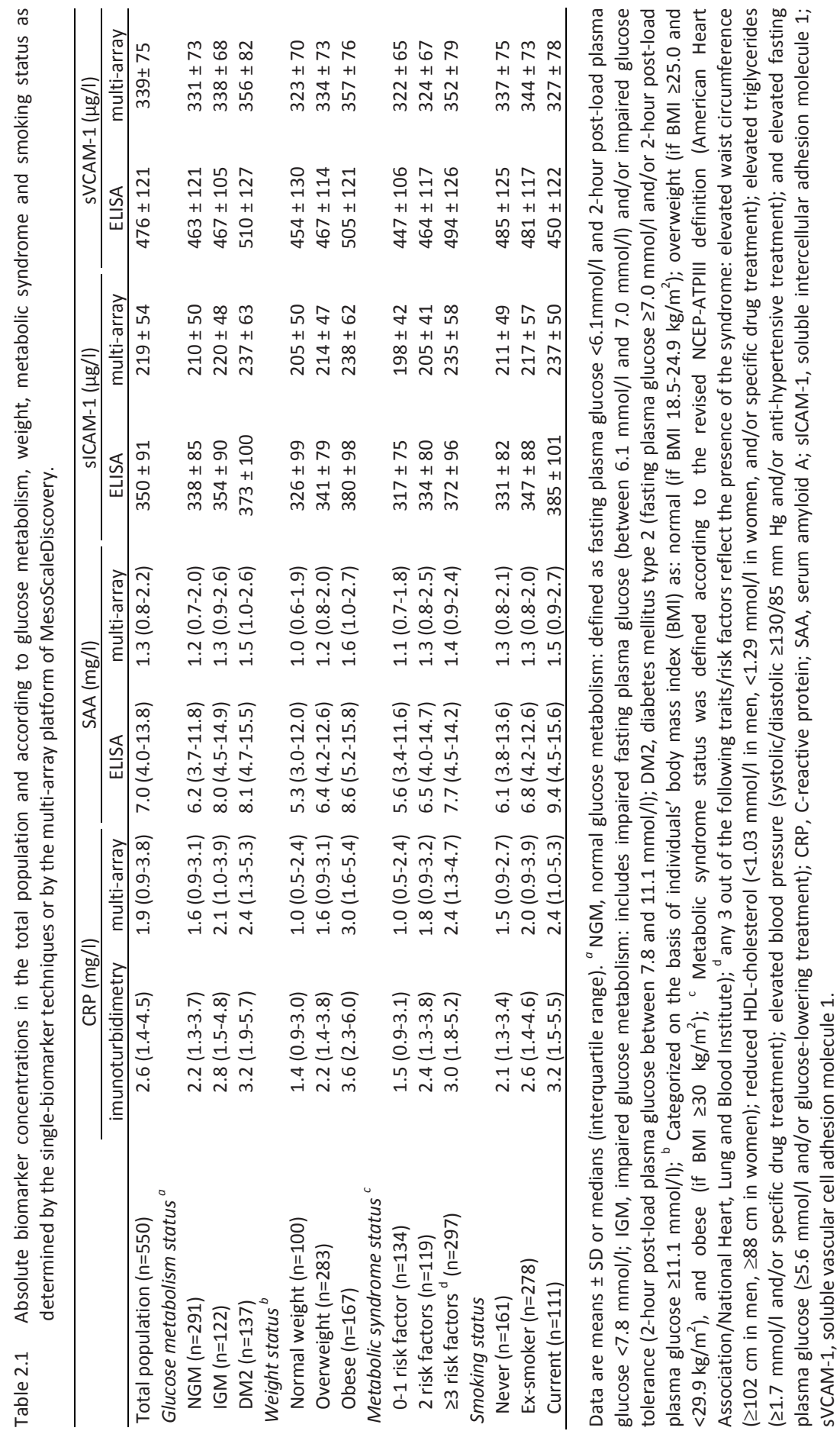




\section{Method comparison}

Despite the very high Pearson's correlation coefficients (i.e. 0.994 for CRP, 0.758 for SAA, 0.816 for sICAM-1 and 0.755 for sVCAM-1) absolute concentrations of biomarkers as obtained by single-biomarker vs. multi-array techniques differed considerably. Indeed, weighted Deming regression analyses for all biomarkers showed significant constant (intercepts) and proportional (slopes) bias between methods such that the absolute mean concentrations of all four biomarkers were lower when measured with the multi-array platform than with the single-biomarker techniques (Figure 2.1A-D, left panels). The above indicates that, when comparing absolute values, realignment of the single-biomarker data to the multi-array data (or viceversa) is thus warranted.

\section{Realignment and agreement}

Realignment of the data as obtained by different methods was therefore conducted with the use of the coefficients retrieved from the Deming regression models (Table 2.2). Bland-Altman plots of the single-biomarker data realigned to the multi-array data (Figure 2.1A-D, right panels) showed that no obvious relation of differences between methods with their mean was present. For all biomarkers, except SAA, Bland-Altman plots confirmed the removal of systematic bias after the realignment [all mean values for differences between methods around 0 (Figure 2.1A, 2.1C and 2.1D, right panels)]. For SAA, a systematic difference between ELISA and multi-array data still persisted after the realignment [about $15 \%$, i.e. $\mathrm{e}^{0.145}$ as compared to their mean (Figure 2.1B, right panel)]. In addition, the equations applied for the realignment (Table 2.2) resulted in similar distributions of single-biomarker and multi-array data (Figure 2.2). The resulting ICCS between single-biomarker (realigned) and multi-array data were 0.996 for CRP, 0.711 for SAA, 0.895 for sICAM-1 and 0.858 for SVCAM-1.

\section{Method performance across different cardiovascular risk groups}

Concentrations of all biomarkers, as measured by single-biomarker or multi-array methods (expressed as Z-scores), increased significantly across categories of glucose metabolism, weight, metabolic syndrome and smoking status (all p-trends $\leq 0.028$, except for sVCAM-1 and smoking status), independently of sex, age, eGFR and prior CVD (Table 2.3). Importantly, the patterns of associations between RFs level and individual biomarker concentrations did not differ by method of detection [all $p$-values for group*method interaction were $>0.05$, except for metabolic syndrome status and $\log _{\mathrm{e}} \mathrm{CRP}$ ( $\mathrm{p}$-value $=0.002$ )] (Table 2.3 ).

These results did not materially change, when the analyses were repeated excluding individuals with CRP values $>10 \mathrm{mg} / \mathrm{l}$, likely to indicate an acute inflammatory response (data not shown). ${ }^{9,17,18}$ 
Weighted Deming Regression
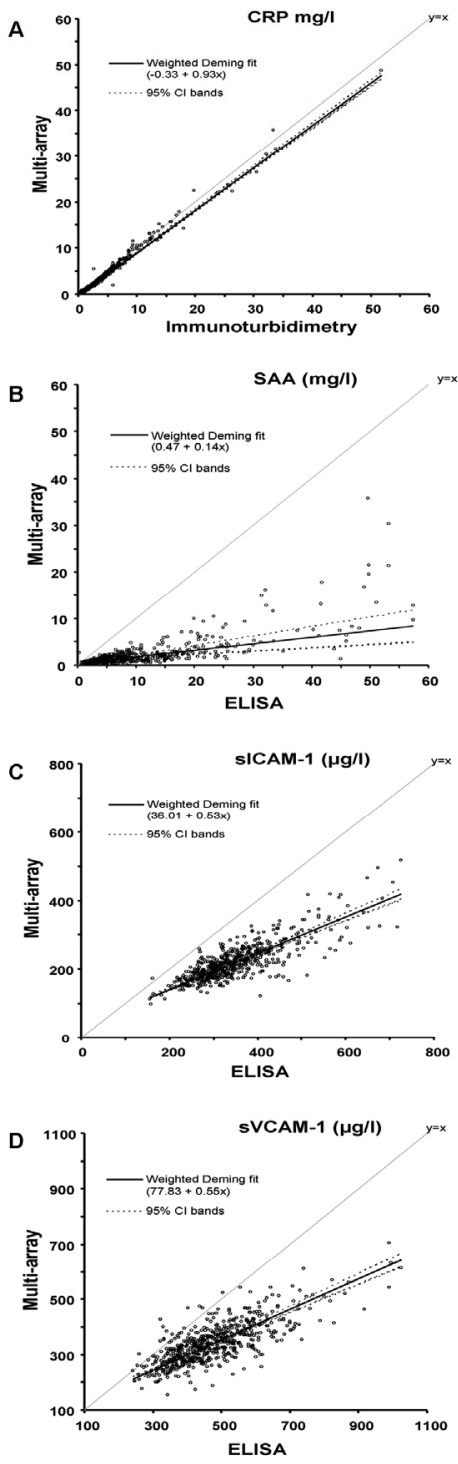

\section{Bland-Altman plots}

$\log _{e} C R P$

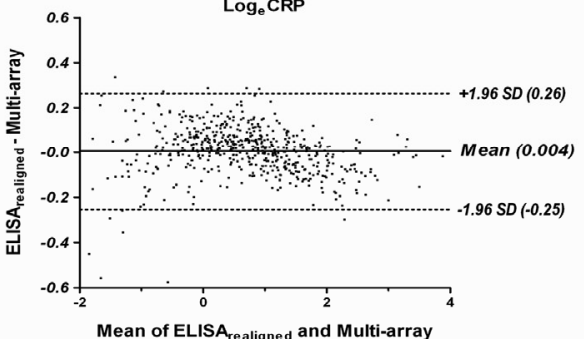

$\log _{\mathrm{e}} \mathrm{SAA}$
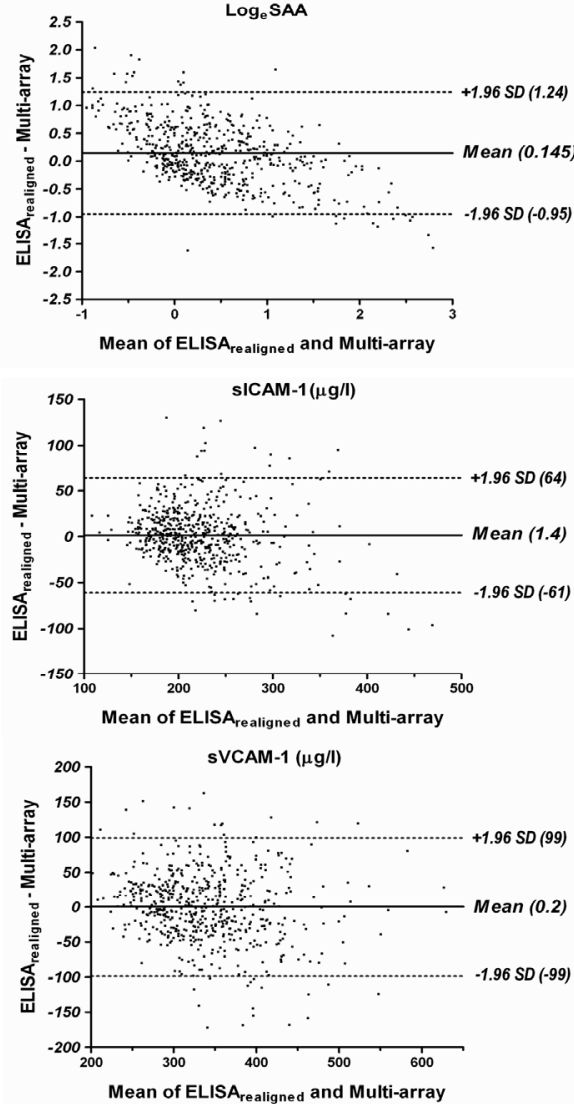

Figure 2.1 Left panel: comparison of biomarker concentrations between the single-biomarker techniques and the multi-array platform by weighted Deming regression; Right panel: Bland-Altman plots of the differences between the two methods; (A) CRP, C-reactive protein ( $n=566)$; (B) SAA, serum amyloid $A$ ( $n=563)$; (C) sICAM-1, soluble intercellular adhesion molecule $1(n=566)$; and (D) sVCAM-1, soluble vascular cell adhesion molecule $1(n=567)$. For $\log _{e}$ transformed data limits of agreement can be converted to original units by anti-log calculation (e.g. for CRP, e ${ }^{0.25}$ and $\mathrm{e}^{0.26}$ which are equivalent to 0.78 and 1.30 times or $22 \%$ below or $30 \%$ above the long axis of CRP, respectively). 
Table $2.2 \quad$ Weighted Deming regression.

\begin{tabular}{|c|c|c|c|c|c|c|c|c|}
\hline \multirow[t]{3}{*}{ Variable } & \multirow[t]{3}{*}{$\mathrm{n}$ pairs } & \multicolumn{4}{|c|}{ Regression equations (weighted Deming) } & \multirow{2}{*}{\multicolumn{2}{|c|}{ slope }} & \multirow{3}{*}{ Sylx } \\
\hline & & \multirow[t]{2}{*}{$\mathrm{Y}$} & \multirow[t]{2}{*}{$x$} & \multicolumn{2}{|c|}{ intercept } & & & \\
\hline & & & & $\alpha$ & $95 \% \mathrm{Cl}$ & $\beta$ & $95 \% \mathrm{Cl}$ & \\
\hline $\begin{array}{l}\text { CRP } \\
(\mathrm{mg} / \mathrm{l})\end{array}$ & 566 & $\mathrm{CRP}_{\mathrm{MA}}$ & $\mathrm{CRP}_{\text {ITв }}$ & -0.33 & $-0.35 ;-0.31$ & 0.93 & $0.91 ; 0.94$ & 0.102 \\
\hline $\begin{array}{l}\text { SAA } \\
(\mathrm{mg} / \mathrm{l})\end{array}$ & 563 & $\mathrm{SAA}_{\mathrm{MA}}$ & SAA $\mathrm{ELISA}_{\text {E }}$ & 0.47 & $0.05 ; 0.90$ & 0.14 & $0.07 ; 0.20$ & 0.239 \\
\hline $\begin{array}{l}\text { sICAM-1 } \\
(\mu \mathrm{g} / \mathrm{l})\end{array}$ & 566 & sICAM-1 $1_{\mathrm{MA}}$ & sICAM-1 $1_{\text {ELISA }}$ & 36.01 & $24.63 ; 47.39$ & 0.53 & $0.49 ; 0.56$ & 0.104 \\
\hline $\begin{array}{l}\text { sVCAM-1 } \\
(\mu \mathrm{g} / \mathrm{l})\end{array}$ & 567 & sVCAM-1 $1_{\mathrm{MA}}$ & sVCAM-1 $1_{\text {ELISA }}$ & 77.83 & $58.90 ; 96.75$ & 0.55 & $0.51 ; 0.59$ & 0.124 \\
\hline $\begin{array}{l}\text { CRP } \\
(\mathrm{mg} / \mathrm{l})\end{array}$ & 566 & $\mathrm{CRP}_{\text {Iтв }}$ & $\mathrm{CRP}_{\mathrm{MA}}$ & 0.35 & $0.34 ; 0.37$ & 1.08 & $1.06 ; 1.10$ & 0.110 \\
\hline $\begin{array}{l}\text { SAA } \\
(\mathrm{mg} / \mathrm{l})\end{array}$ & 563 & SAA $A_{\text {ELISA }}$ & $\mathrm{SAA}_{\mathrm{MA}}$ & -3.46 & $-7.37 ; 0.45$ & 7.33 & $4.40 ; 10.25$ & 1.751 \\
\hline $\begin{array}{l}\text { sICAM-1 } \\
(\mu \mathrm{g} / \mathrm{l})\end{array}$ & 566 & SICAM-1 $1_{\text {ELISA }}$ & sICAM-1 $1_{\mathrm{MA}}$ & -68.49 & $-94.71 ;-42.27$ & 1.90 & $1.77 ; 2.03$ & 0.197 \\
\hline $\begin{array}{l}\text { sVCAM-1 } \\
(\mu \mathrm{g} / \mathrm{l})\end{array}$ & 567 & sVCAM-1 $1_{\text {ELISA }}$ & sVCAM-1 $1_{\mathrm{MA}}$ & -141.52 & $-186.54 ;-96.49$ & 1.82 & $1.68 ; 1.96$ & 0.225 \\
\hline
\end{tabular}

Data are intercepts $(\alpha)$ and (slopes) $\beta$ of the Deming regression equation, which all differed significantly from 0 and 1, respectively, as indicated by their $95 \% \mathrm{Cl}$; rejection of the hypothesis that $\alpha=0$ means that the two methods differ at least by a constant amount; rejection of the hypothesis that $\beta=1$ implies that there is a proportional difference between methods; Sylx are standard deviations of the residuals; Upper panel: these equations are used as cross-validation equations to realign single-biomarker data (ITB, immunoturbidimetry; or ELISA) to multi-array (MA) data. Lower panel: these equations are used as crossvalidation equations to realign multi-array (MA) data to single-biomarker data (ITB, immunoturbidimetry; or ELISA). CRP, C-reactive protein; SAA, serum amyloid A; sICAM-1, soluble intercellular adhesion molecule 1; sVCAM-1, soluble vascular cell adhesion molecule 1.

\section{Additional analyses}

A key step in biochemical tests comparison is to ascertain whether the level of agreement between methods is acceptable from a clinical standpoint. ${ }^{40}$ For CRP, values $<1,1-3$, and $>3 \mathrm{mg} / \mathrm{l}$ have been proposed to identify individuals at low, intermediate and high-risk for incident CVD, respectively, whereas such values are lacking for the other biomarkers examined herein. This impairs the appreciation of the clinical relevance of the limits of agreement between methods obtained for these biomarkers (Figure 2.1B-D, right panels). ${ }^{8,9}$ Still, for CRP we could ascertain that, on the basis of immunoturbidimetry, $12.9 \%$ of the CODAM Study population would be classified at 'low risk', $46.4 \%$ at 'intermediate risk' and $40.7 \%$ at 'high-risk'; on the basis of the multi-array platform these numbers would be $28.0,39.5$ and $32.5 \%$, respectively (Cohen's $\kappa=0.641$, which is a measure of agreement for categorical data; overall concordance rate of $76.7 \%$ ). After realignment of the immunoturbidimetry to the multi-array data and vice versa, the agreement between methods increased 
considerably (Cohen's $\kappa$ of 0.931 and 0.946 and concordance rates of 95.4 and $96.7 \%$, respectively - Table 2.4).

\section{Box-and-whisker plots}

A

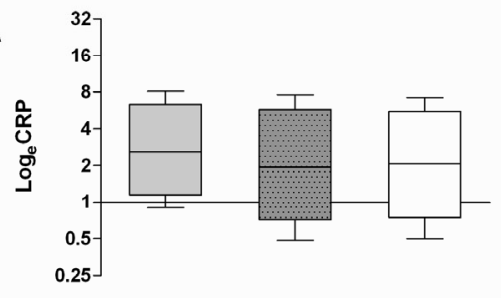

$\underline{\underline{m}}$

$\underline{\underline{s}}$

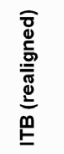

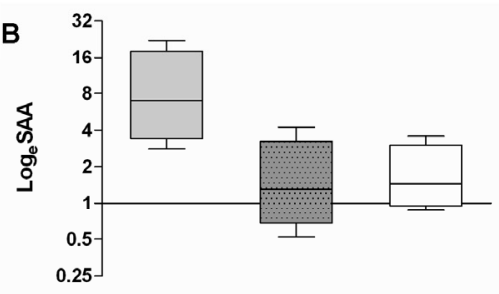

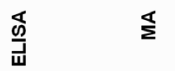

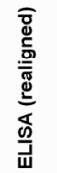

C

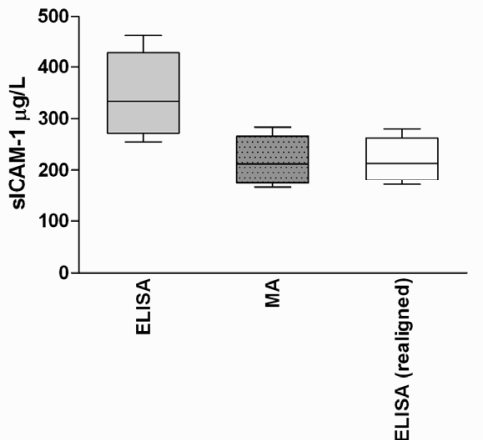

D

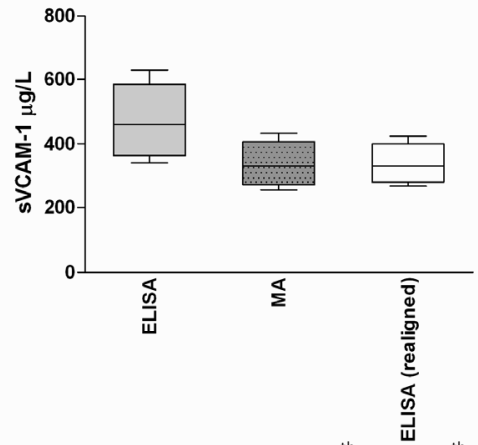

Figure 2.2 Box-and-whisker plots showing median and interquartile (box) and $10^{\text {th }}$ and $90^{\text {th }}$ centiles (whiskers) for each biomarker, before and after realignment to current (multi-array) concentrations. (A) CRP, C-reactive protein $(n=566)$; (B) SAA, serum amyloid A ( $n=563)$; (C) sICAM-1, soluble intercellular adhesion molecule $1(n=566)$; and (D) SVCAM-1, soluble vascular cell adhesion molecule 1 ( $n=567$ ); ITB, immunoturbidimetry; MA, multi-array. 


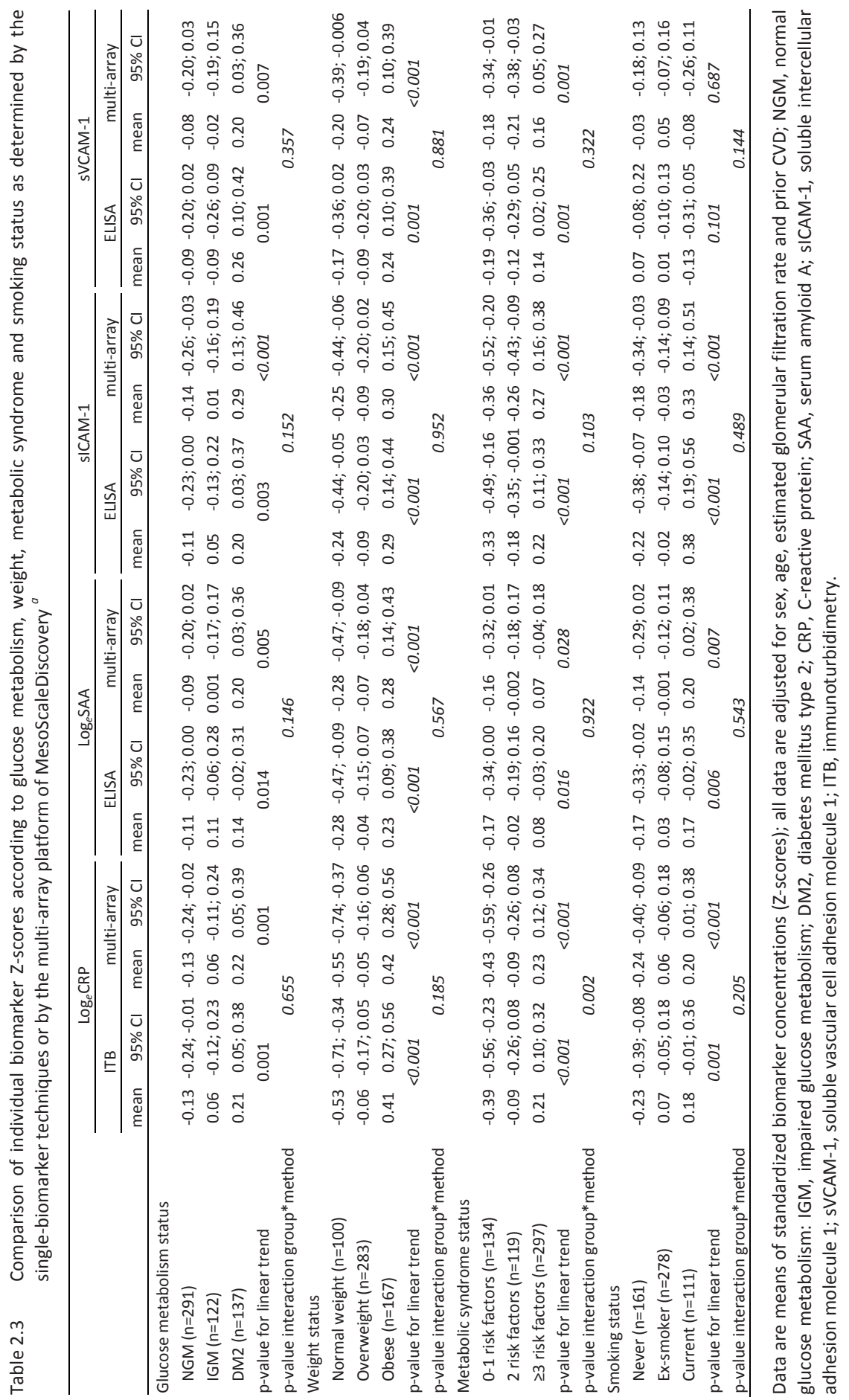


Table 2.4 Agreement in risk level assignment on the basis of CRP obtained by immunoturbidimetry and the multi-array platform.

\begin{tabular}{|c|c|c|c|c|c|c|c|c|c|c|c|c|c|}
\hline \multirow[t]{2}{*}{ CRP } & \multirow[b]{2}{*}{ level } & \multicolumn{4}{|c|}{ Multi-array platform } & \multirow[b]{2}{*}{$\begin{array}{c}\text { concordance } \\
(\%)\end{array}$} & \multirow[b]{2}{*}{$\kappa$} & \multicolumn{4}{|c|}{$\begin{array}{l}\text { Multi-array platform } \\
\text { realigned to } \\
\text { immunoturbidimetry }\end{array}$} & \multirow[b]{2}{*}{$\begin{array}{c}\text { concordance } \\
(\%)\end{array}$} & \multirow[b]{2}{*}{$\kappa$} \\
\hline & & $\begin{array}{c}<1 \\
\mathrm{mg} / \mathrm{l} \\
(\%) \\
\end{array}$ & $\begin{array}{c}1-3 \\
\mathrm{mg} / \mathrm{l} \\
(\%) \\
\end{array}$ & $\begin{array}{c}>3 \\
\mathrm{mg} / \mathrm{l} \\
(\%) \\
\end{array}$ & $\begin{array}{c}\text { total } \\
(\%)\end{array}$ & & & $\begin{array}{c}<1 \\
\mathrm{mg} / \mathrm{l} \\
(\%) \\
\end{array}$ & $\begin{array}{c}1-3 \\
\mathrm{mg} / \mathrm{l} \\
(\%) \\
\end{array}$ & $\begin{array}{c}>3 \\
\mathrm{mg} / \mathrm{l} \\
(\%) \\
\end{array}$ & $\begin{array}{l}\text { total } \\
(\%)\end{array}$ & & \\
\hline Immuno- & $<1 \mathrm{mg} / \mathrm{l}$ & 12.9 & 0.0 & 0.0 & 12.9 & & 0.641 & 12.5 & 0.4 & 0.0 & 12.9 & & 0.946 \\
\hline \multirow[t]{4}{*}{ turbidimetry } & $1-3 \mathrm{mg} / \mathrm{l}$ & 15.1 & 31.3 & 0.0 & 46.4 & & & 0.5 & 45.5 & 0.4 & 46.4 & & \\
\hline & $>3 \mathrm{mg} / \mathrm{l}$ & 0.0 & 8.2 & 32.5 & 40.7 & & & 0.0 & 2.0 & 38.7 & 40.7 & & \\
\hline & total & 28.0 & 39.5 & 32.5 & 100.0 & & & 13.1 & 47.8 & 39.1 & 100.0 & & \\
\hline & & & & & & 76.7 & & & & & & 96.7 & \\
\hline Immuno- & $<1 \mathrm{mg} / \mathrm{l}$ & 26.0 & 0.4 & 0.0 & 26.4 & & 0.931 & - & - & - & - & & - \\
\hline turbidimetry & $1-3 \mathrm{mg} / \mathrm{l}$ & 2.0 & 37.6 & 0.7 & 40.4 & & & - & - & - & - & & \\
\hline realigned to & $>3 \mathrm{mg} / \mathrm{l}$ & 0.0 & 1.5 & 31.8 & 33.3 & & & - & - & - & - & & \\
\hline \multirow[t]{2}{*}{ multi-array } & total & 28.0 & 39.5 & 32.5 & 100.0 & & & - & - & - & - & & \\
\hline & & & & & & 95.4 & & & & & & - & \\
\hline
\end{tabular}

$\kappa$, Cohen's kappa (measure of agreement for categorical data); -, not applicable.

\section{Discussion}

The present study has three main findings. First, the absolute concentrations of CRP, SAA, sICAM-1 and sVCAM-1 differed significantly between the single-biomarker techniques and the multi-array platform of MSD. Second, equations retrieved by weighted Deming regression enabled proper realignment of the data to overcome these absolute differences. Finally, the overall pattern of associations between levels of the individual biomarkers with glucose metabolism, weight, metabolic syndrome and smoking status did not differ by method of detection. This is the first study that has examined and cross-validated, in a large ongoing cohort study, measurements of biomarkers of low-grade inflammation by means of single-biomarker techniques and the multi-array platform of MSD.

Our results are in line with a previous study, which suggested that data measured with single-biomarker techniques and data measured with the multi-array platform cannot be combined without appropriate realignment of the data as this would distort epidemiological associations. ${ }^{23}$ In our study, the absolute concentrations of all four biomarkers were lower when measured with the multi-array platform than with the single-biomarker techniques. It should be emphasized, however, that the absolute concentration of each biomarker is based on the standards provided by the commercial kits and the lack of international standardization among these may therefore explain the differences between methods. ${ }^{9}$ Although CRP reference materials exist, bias attributed to standardization remains due to the fact that reference materials were developed to distinguish between CRP values below $10 \mathrm{mg} / \mathrm{l}$, from 10 to $40 \mathrm{mg} / \mathrm{l}$ and above $40 \mathrm{mg} / \mathrm{l}$, whereas current assays aim for 
accurate and reproducible detection down to $0.3 \mathrm{mg} / \mathrm{l} .{ }^{18}$ Also according to the Centers for Disease Control and Prevention and the American Heart Association laboratory science discussion group, further standardization efforts are therefore required as measurements of absolute biomarker concentrations are of paramount importance for direct comparison between studies using different methods and for definition of clinical cutoff values. ${ }^{18}$ Nevertheless, in the present study we were able to appropriately realign the data to overcome the absolute differences between both methods. Thus, the introduction of a multi-array platform in an ongoing cohort study may be implemented without impairing the investigation of within-subject changes in biomarker concentrations over the course of time. This was enabled by re-assaying all the baseline samples with the new multi-array method. In addition, we show that the agreement in risk level assignment on the basis of CRP levels $\left(<1,1-3\right.$, and $\left.>3 \mathrm{mg} / \mathrm{I}^{8,9}\right)$ is very high after realignment. It remains, however, that subjects' risk-level assignment depends on the method used for CRP assessment, and that if this were done on the basis of MSD readings, less individuals from the CODAM Study would be considered to be at high-risk than if this were done on the basis of immunoturbidimetry readings. However, to establish which method is superior in risk prediction further studies are warranted.

Another option to directly compare individual biomarker levels between methods (but also between clinical studies) is by transformation of data to Z-scores, especially if realignment equations are lacking. By Z-score transformation, between-subjects ranking in terms of their biomarkers levels are preserved within the population. The present study shows that Z-scores of CRP, SAA, sICAM-1, sVCAM-1 differed across categories of glucose metabolism, weight, metabolic syndrome and smoking status in a similar fashion irrespective of the method of detection. Although it is evident that a high correlation between assays will result in identical associations, these results, illustrate and emphasize that, despite absolute differences, the relative differences are comparable between the single-biomarker techniques and the multi-array platform.

Taken together, our findings suggest that the multi-array platform of MSD could potentially replace the single-biomarker techniques for the detection of multiple biomarkers in large ongoing and future clinical studies aiming at the investigation of the role of low-grade inflammation in the aetiology of CVD, though careful validation would be required.

Furthermore, the multi-array platform of MSD has several practical advantages over the well-established single-biomarker techniques for biomarker detection, although CRP assays are generally automated ${ }^{18}: 1$ ) it has simple operating procedures; 2 ) it has a higher sensitivity and greater detection range, which eliminates multiple dilutions and freeze and thaw cycles per sample; 3 ) it allows determination of four (or more) biomarkers simultaneously, improving the labour-efficiency, and due costs; and 4) it uses a small sample volume $(5 \mu \mathrm{l}$ instead of $50 \mu \mathrm{l}$ for the detection of these four markers), which is useful in clinical and epidemiological studies. 
The present study has some limitations. First, with the single-biomarker techniques, CRP was measured in serum and SAA, sVCAM-1 and sICAM-1 were measured in plasma, whereas with the multi-array platform all biomarkers were measured in plasma. This may, in part, explain the differences between methods in absolute concentrations of CRP, since a different matrix might effect detection. Furthermore, the measurement of biomarkers by the single-biomarker techniques and the multiarray platform were performed $\sim 7$ years apart, which could also have contributed to an underestimation of absolute biomarker concentrations by the multi-array platform. However, because storage time of samples was the same for all study individuals, if anything: 1) this underestimation was likely systematic and properly incorporated in the realignment equations; and 2) could not have affected the relative differences in biomarkers across different levels of subjects' cardiovascular RFs. Second, we showed realignment equations to enable transition of 'old' to 'new' methods within our ongoing cohort study (and vice versa). However, the results were shown in detail for single-biomarker data realigned to multi-array data. This way of presentation facilitates future comparisons of those biomarkers measured with the multi-array platform at follow-up examinations within this ongoing cohort study. However, any other cohort study should calculate realignment equations within their own data. These may be susceptible to lot-to-lot variation, although in our laboratory the lot-tolot variation between multi-array assays was low for most of the biomarkers. Nevertheless, the measured concentrations will always depend on the standards provided by the commercial kits (for both the single biomarker and multi-array techniques), which have not been satisfactorily standardized internationally.,18

In conclusion, multiple biomarker detection by the 4-plex multi-array platform of MSD including CRP, SAA, SICAM-1 and SVCAM-1 shows comparable results with wellestablished single-biomarker techniques, despite differences in absolute concentrations. Subjects' risk-level assignment therefore depends on the method used. It is, however, uncertain which method is superior in risk prediction. Nevertheless, these biomarkers of low-grade inflammation are associated with glucose metabolism, weight, metabolic syndrome and smoking status, irrespective of the method of detection. In terms of time, effort and quality, this multi-array platform of MSD is an attractive alternative for single-biomarker measurements. Therefore, this platform is a potential tool for the quantification of multiple biomarkers of low-grade inflammation using small sample volume in one single run in large ongoing and future clinical studies. 


\section{References}

1. Borissoff JI, Spronk HM, ten Cate H. The hemostatic system as a modulator of atherosclerosis. N Engl J Med. 2011; 364:1746-1760.

2. Ross R. Atherosclerosis--an inflammatory disease. N Engl J Med. 1999; 340:115-126.

3. Becker A, van Hinsbergh VW, Jager A, Kostense PJ, Dekker JM, Nijpels G, Heine RJ, Bouter LM, Stehouwer CD. Why is soluble intercellular adhesion molecule-1 related to cardiovascular mortality? Eur J Clin Invest 2002; 32:1-8.

4. Blankenberg S, Rupprecht HJ, Bickel C, Peetz D, Hafner G, Tiret L, Meyer J. Circulating cell adhesion molecules and death in patients with coronary artery disease. Circulation. 2001; 104:1336-1342.

5. Kaplan RC, McGinn AP, Baird AE, Hendrix SL, Kooperberg C, Lynch J, Rosenbaum DM, Johnson KC, Strickler HD, Wassertheil-Smoller S. Inflammation and hemostasis biomarkers for predicting stroke in postmenopausal women: the Women's Health Initiative Observational Study. J Stroke Cerebrovasc Dis. 2008; 17:344-355.

6. Ridker PM, Hennekens $\mathrm{CH}$, Buring JE, Rifai N. C-reactive protein and other markers of inflammation in the prediction of cardiovascular disease in women. N Engl J Med. 2000; 342:836-843.

7. Tzoulaki I, Murray GD, Lee AJ, Rumley A, Lowe GD, Fowkes FG. Relative value of inflammatory, hemostatic, and rheological factors for incident myocardial infarction and stroke: the Edinburgh Artery Study. Circulation. 2007; 115:2119-2127.

8. Vasan RS. Biomarkers of cardiovascular disease: molecular basis and practical considerations. Circulation. 2006; 113:2335-2362.

9. Pearson TA, Mensah GA, Alexander RW, Anderson JL, Cannon RO 3rd, Criqui M, Fadl YY, Fortmann SP, Hong Y, Myers GL, Rifai N, Smith SC Jr, Taubert K, Tracy RP, Vinicor F; Centers for Disease Control and Prevention; American Heart Association. Markers of inflammation and cardiovascular disease: application to clinical and public health practice: A statement for healthcare professionals from the Centers for Disease Control and Prevention and the American Heart Association. Circulation. 2003; 107:499-511.

10. Schnabel RB, Larson MG, Yamamoto JF, Kathiresan S, Rong J, Levy D, Keaney JF Jr, Wang TJ, Vasan RS, Benjamin EJ. Relation of multiple inflammatory biomarkers to incident atrial fibrillation. Am J Cardiol. 2009; 104:92-96.

11. Conen D, Ridker PM, Everett BM, Tedrow UB, Rose L, Cook NR, Buring JE, Albert CM. A multimarker approach to assess the influence of inflammation on the incidence of atrial fibrillation in women. Eur Heart J. 2010; 31:1730-1736.

12. Kim HC, Greenland P, Rossouw JE, Manson JE, Cochrane BB, Lasser NL, Limacher MC, Lloyd-Jones DM, Margolis KL, Robinson JG. Multimarker prediction of coronary heart disease risk: the Women's Health Initiative. J Am Coll Cardiol. 2010; 55:2080-2091.

13. Daniels LB, Maisel AS. Multiple marker approach to risk stratification in patients with stable coronary artery disease: to have or have not. Eur Heart J. 2010; 31:2980-2983.

14. Wang TJ. Multiple biomarkers for predicting cardiovascular events: lessons learned. J Am Coll Cardiol. 2010; 55:2092-2095.

15. Gruson D, Bodovitz S. Rapid emergence of multimarker strategies in laboratory medicine. Biomarkers 2010; 15:289-296.

16. Tedgui A, Mallat Z. Cytokines in atherosclerosis: pathogenic and regulatory pathways. Physiol Rev. 2006; 86:515-581.

17. Thanabalasingham $G$, Shah N, Vaxillaire $M$, Hansen $T$, Tuomi $T$, Gašperíková D, Szopa M, Tjora E, James TJ, Kokko P, Loiseleur F, Andersson E, Gaget S, Isomaa B, Nowak N, Raeder H, Stanik J, Njolstad PR, Malecki MT, Klimes I, Groop L, Pedersen O, Froguel P, McCarthy MI, Gloyn AL, Owen KR. A large multi-centre European study validates high-sensitivity C-reactive protein (hsCRP) as a clinical biomarker for the diagnosis of diabetes subtypes. Diabetologia. 2010; 54:2801-2810.

18. Myers GL, Rifai N, Tracy RP, Roberts WL, Alexander RW, Biasucci LM, Catravas JD, Cole TG, Cooper GR, Khan BV, Kimberly MM, Stein EA, Taubert KA, Warnick GR, Waymack PP; CDC; AHA. CDC/AHA Workshop on Markers of Inflammation and Cardiovascular Disease: Application to Clinical and Public Health Practice: report from the laboratory science discussion group. Circulation. 2004; 110:e545549. 
19. Marchese RD, Puchalski D, Miller P, Antonello J, Hammond O, Green T, Rubinstein LJ, Caulfield MJ, Sikkema D. Optimization and validation of a multiplex, electrochemiluminescence-based detection assay for the quantitation of immunoglobulin $\mathrm{G}$ serotype-specific antipneumococcal antibodies in human serum. Clin Vaccine Immunol. 2009; 16:387-396.

20. Oh ES, Mielke MM, Rosenberg PB, Jain A, Fedarko NS, Lyketsos CG, Mehta PD. Comparison of conventional ELISA with electrochemiluminescence technology for detection of amyloid-beta in plasma. J Alzheimers Dis. 2010; 21:769-773.

21. Prabhakar U, Eirikis E, Davis HM. Simultaneous quantification of proinflammatory cytokines in human plasma using the LabMAP assay. J Immunol Methods. 2002; 260:207-218.

22. Dupont NC, Wang K, Wadhwa PD, Culhane JF, Nelson EL. Validation and comparison of luminex multiplex cytokine analysis kits with ELISA: determinations of a panel of nine cytokines in clinical sample culture supernatants. J Reprod Immunol. 2005; 66:175-191.

23. de Koning L, Liptak C, Shkreta A, Bradwin G, Hu FB, Pradhan AD, Rifai N, Kellogg MD. A multiplex immunoassay gives different results than singleplex immunoassays which may bias epidemiologic associations. Clin Biochem. 2012; 45:848-851.

24. Steffes M, Cleary P, Goldstein D, Little R, Wiedmeyer HM, Rohlfing C, England J, Bucksa J, Nowicki M. Hemoglobin A1c measurements over nearly two decades: sustaining comparable values throughout the Diabetes Control and Complications Trial and the Epidemiology of Diabetes Interventions and Complications study. Clin Chem. 2005; 51:753-758.

25. Cull CA, Manley SE, Stratton IM, Neil HA, Ross IS, Holman RR, Turner RC, Matthews DR. Approach to maintaining comparability of biochemical data during long-term clinical trials. Clin Chem. 1997; 43:1913-1918.

26. Maahs DM, Jalal D, McFann K, Rewers M, Snell-Bergeon JK. Systematic shifts in cystatin C between 2006 and 2010. Clin J Am Soc Nephrol. 2011; 6:1952-1955.

27. Selvin E, Coresh J, Zhu H, Folsom A, Steffes MW. Measurement of HbA1c from stored whole blood samples in the Atherosclerosis Risk in Communities study. J Diabetes. 2010; 2:118-124.

28. Kruijshoop M, Feskens EJ, Blaak EE, de Bruin TW. Validation of capillary glucose measurements to detect glucose intolerance or type 2 diabetes mellitus in the general population. Clin Chim Acta. 2004; 341:33-40.

29. Du H, van der A DL, van Bakel MM, van der Kallen CJ, Blaak EE, van Greevenbroek MM, Jansen EH, Nijpels G, Stehouwer CD, Dekker JM, Feskens EJ. Glycemic index and glycemic load in relation to food and nutrient intake and metabolic risk factors in a Dutch population. Am J Clin Nutr. 2008; 87:655661.

30. Jacobs M, van Greevenbroek MM, van der Kallen CJ, Ferreira I, Blaak EE, Feskens EJ, Jansen EH, Schalkwijk CG, Stehouwer CD. Low-grade inflammation can partly explain the association between the metabolic syndrome and either coronary artery disease or severity of peripheral arterial disease: the CODAM study. Eur J Clin Invest. 2009; 39:437-444.

31. Jacobs M, van Greevenbroek MM, van der Kallen CJ, Ferreira I, Blaak EE, Feskens EJ, Jansen EH, Schalkwijk CG, Stehouwer CD. The association between the metabolic syndrome and peripheral, but not coronary, artery disease is partly mediated by endothelial dysfunction: the CODAM study. Eur J Clin Invest. 2010; 41:167-175.

32. Thewissen MM, Damoiseaux JG, Duijvestijn AM, van Greevenbroek MM, van der Kallen CJ, Feskens EJ, Blaak EE, Schalkwijk CG, Stehouwer CD, Cohen Tervaert JW, Ferreira I. Abdominal fat mass is associated with adaptive immune activation: The CODAM study. Obesity (Silver Spring). 2010; 19:1690-1698.

33. van Greevenbroek MM, Jacobs M, van der Kallen CJ, Blaak EE, Jansen EH, Schalkwijk CG, Feskens EJ, Stehouwer CD. Human plasma complement C3 is independently associated with coronary heart disease, but only in heavy smokers (the CODAM study). Int J Cardiol. 2012; 154:158-162.

34. Levey AS, Coresh J, Greene T, Marsh J, Stevens LA, Kusek JW, Van Lente F; Chronic Kidney Disease Epidemiology Collaboration. Expressing the Modification of Diet in Renal Disease Study equation for estimating glomerular filtration rate with standardized serum creatinine values. Clin Chem. 2007; 53:766-772.

35. van Bussel BC, Schouten F, Henry RM, Schalkwijk CG, de Boer MR, Ferreira I, Smulders YM, Twisk JW, Stehouwer CD. Endothelial dysfunction and low-grade inflammation are associated with greater arterial stiffness over a 6-year period. Hypertension. 2011; 58:588-595. 
36. van Bussel BC, Henry RM, Schalkwijk CG, Dekker JM, Nijpels G, Stehouwer CD. Low-grade inflammation, but not endothelial dysfunction, is associated with greater carotid stiffness in the elderly: the Hoorn Study. J Hypertens. 2012; 30:744-752.

37. Linnet K. Performance of Deming regression analysis in case of misspecified analytical error ratio in method comparison studies. Clin Chem. 1998; 44:1024-1031.

38. Martin RF. General deming regression for estimating systematic bias and its confidence interval in method-comparison studies. Clin Chem. 2000; 46:100-104.

39. Bland JM, Altman DG. Statistical methods for assessing agreement between two methods of clinical measurement. Lancet. 1986; 1:307-310.

40. Twomey PJ. How to use difference plots in quantitative method comparison studies. Ann Clin Biochem. 2006; 43:124-129.

41. de Vet HC, Terwee CB, Knol DL, Bouter LM. When to use agreement versus reliability measures. J Clin Epidemiol. 2006; 59:1033-1039. 


\section{Chapter \\ 3}

Control systems approach to assessment of flowmediated dilation improves the detection of changes in local brachial artery dilation after an oral glucose load

FCG van Bussel* , BCT van Bussel* , APG Hoeks, J Op 't Roodt, RMA Henry, I Ferreira, FHM Vanmolkot, CG Schalkwijk, CDA Stehouwer, KD Reesink *Both authors contributed equally Submitted for publication 


\section{Abstract}

Brachial artery flow-mediated dilation is aimed at normalization of local wall shear stress under varying blood flow conditions. Blood flow velocity and vessel diameter are continuous and opposing influences that modulate wall shear stress. Considering these physical interrelationships within this control system, we derived an $\mathrm{FMD}_{v}$ index to quantify specifically changes in flow-mediated dilation at brachial artery level occurring with oral glucose challenge. In 22 fasting presumed healthy men, we assessed intra- and inter-session reproducibilities of two indices $\mathrm{pFMD}_{\mathrm{v}}$ and $\mathrm{MFMD}_{\mathrm{v}}$ considering the relative peak or relative mean hyperaemic change in velocity as shear stress stimulus, respectively. We compared the tracking performance of these $\mathrm{FMD}_{v}$ indices with existing indices [i.e. the relative peak diameter increase (\%FMD), the peak to baseline diameter ratio $\left(D_{\text {peak }} / D_{\text {base }}\right)$ and the relative peak diameter increase normalized to the full area under the curve of blood flow velocity with hyperaemia

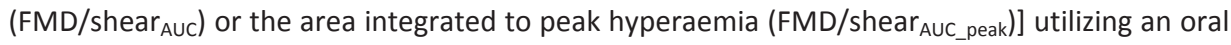
glucose challenge, inducing an acute reduction in flow-mediated dilation, which was assessed 30 and 75 minutes after intake. Inter-session and intra-session reproducibilities for $\mathrm{pFMD}_{\mathrm{v}}$, $\mathrm{mFMD}_{\mathrm{v}}$ and \%FMD were comparable (intra-class correlation coefficients within 0.521-0.677 range). Both $\mathrm{pFMD}_{v}$ and $\mathrm{mFMD}_{\mathrm{v}}$ showed more clearly a reduction after glucose load (relative reduction of $\sim 45 \%, p \leq 0.001$ ) than other indices (\% given are relative reductions): \%FMD ( $11 \%$, $\mathrm{p} \geq 0.074) ; \mathrm{D}_{\text {peak }} / \mathrm{D}_{\text {base }}(\sim 11 \%, \mathrm{p} \geq 0.074) ; \mathrm{FMD} /$ shear $_{\text {AUC_peak }}(\sim 20 \%, \mathrm{p} \geq 0.016)$ and $\mathrm{FMD} /$ shear $_{\text {AUC }}$ $(\sim 38 \%, p \leq 0.038)$. A control systems approach to quantification improves the detection of flowmediated dilation changes at brachial artery level after glucose load. The $\mathrm{FMD}_{\mathrm{v}}$ approach may be particularly useful in acute pharmacological or physiological intervention studies aimed at discriminating effects on conduit artery flow-mediated dilation and those on the microcirculation. 


\section{Introduction}

Dilation of vascular beds in response to an increased blood volume flow is an essential mechanism in mammalian (early) development and in adult circulatory adaptation. ${ }^{1-3}$ Current insight is that shear stress, sensed and transduced by the glycocalyxendothelium apparatus, plays a major role in the acute and long-term adaptation of vascular diameter. ${ }^{1,3}$ Dysfunction of the endothelial mechano-transduction is commonly assessed non-invasively by measuring brachial artery dilation ${ }^{4-6}$ as elicited by a hyperaemic flow-stimulus, commonly quantified as the relative peak increase in brachial artery diameter (\%FMD). ${ }^{7-11}$ A low \%FMD has been associated with (cardio)vascular events ${ }^{12,13}$ and is considered a subclinical marker of vascular dysfunction as may occur in hypertension, diabetes, and hypercholesterolaemia., ${ }^{4,9}$

Despite improvements in the measurement of brachial artery \% $\mathrm{FMD}^{6-8,14}$, its use in vascular risk assessment and clinical follow-up remains limited due to technical challenges and (related) poor reproducibility. ${ }^{14-18}$ The latter may be explained by the fact that the magnitude of the hyperaemic flow-stimulus (which is utilized to elicit the dilatory response) is commonly not accounted for. ${ }^{19-26}$ The hyperaemic flow-stimulus, as a product of peripheral vascular reactivity, is subject to health status ${ }^{9,10}$ and may by itself be modulated by the interventions studied. ${ }^{27,28}$ Although several ways to incorporate stimulus magnitude have been proposed and advocated ${ }^{9,11,29,30}$, the question remains how to account for stimulus magnitude when quantifying flowmediated dilation. ${ }^{31}$

Since the mean wall shear stress is directly related to the ratio of mean blood flow velocity and vessel diameter ${ }^{11,32,33}$, these two variables have opposing influences on wall shear stress. We hypothesize that the continuous balance between these influences is a more accurate measure to quantify flow-mediated dilation and especially changes thereof at the brachial artery level.

Time averaged blood flow through the brachial artery can be approximated by an incompressible liquid with constant viscosity moving within a straight, long tube, thus having a parabolic velocity distribution. These presumptions are valid for flowmediated dilation assessment, where we consider a time-scale of many times the cardiac cycle and consequently measure time-average blood flow velocity and vessel diameter. According to Poiseuille's law the flowing blood exerts a frictional stress on the inner lining of the vessel, termed wall shear stress $\left(\tau_{w}\right)$. For a given blood viscosity $(\eta)$ and a presumed parabolic velocity profile, brachial artery $\tau_{w}$ is a function of the local centre-stream blood flow velocity (v) and diameter (D), according to ${ }^{3}$ :

$$
\mathrm{T}_{\mathrm{w}}=\frac{8 \eta \mathrm{v}}{\mathrm{D}} \quad \text { (eq. 1) }
$$


In a first order Taylor approximation, any change in $\tau_{w}$ can be expressed as a difference equation with respect to velocity and diameter:

$$
\Delta \mathrm{T}_{\mathrm{w}}=\frac{8 \eta}{\mathrm{D}_{\text {base }}} \Delta \mathrm{v}-\frac{8 \eta \mathrm{v}_{\text {base }}}{\mathrm{D}_{\text {base }}^{2}} \Delta \mathrm{D}
$$

The first term on the right hand side of equation 2 reflects the change in wall shear stress evoked by a change $\left(\Delta v=v-v_{\text {base }}\right)$ in blood flow velocity, while the second term reflects the opposite change in wall shear stress evoked by a change ( $\left.\triangle D=D-D_{\text {base }}\right)$ in diameter. The efficacy with which the diameter change counteracts any velocity induced change in wall shear stress is then given by the ratio of the corresponding terms:

$$
\mathrm{FMD}_{\mathrm{v}}=\frac{8 \eta \mathrm{v}_{\text {base }} \Delta \mathrm{D} / \mathrm{D}_{\text {base }}^{2}}{8 \eta \Delta \mathrm{v} / \mathrm{D}_{\text {base }}}=\frac{\Delta \mathrm{D} / \mathrm{D}_{\text {base }}}{\Delta \mathrm{v} / \mathrm{v}_{\text {base }}}
$$

From the above equation it follows that this velocity normalized index (i.e. $F \mathrm{MD}_{\mathrm{v}}$ ) has a lowest value of 0 in the absence of a diameter response $(\Delta D=0)$. In contrast, increasing values of $\mathrm{FMD}_{\mathrm{v}}$ indicate more effective restoration of mean wall shear stress to baseline, despite the increased volume flow through the artery.

Equation 3 is derived for a steady state situation where the hyperaemic state is compared with the baseline. The selection of appropriate parameters for the transient case is less obvious and depends on the anticipated reproducibility. Since the change in mean diameter is limited and might be more susceptible to baseline drift, we, as is commonly done, also opted for the peak change in diameter. On the other hand, the velocity exhibits a marked change with hyperaemia, permitting the selection of either the peak or mean change in velocity, resulting in the following specific indices (see Figure 3.1 for definition of parameters):

$$
\mathrm{pFMD}_{\mathrm{v}}=\frac{\left(\mathrm{D}_{\text {peak }}-\mathrm{D}_{\text {base }}\right) / \mathrm{D}_{\text {base }}}{\left(\mathrm{v}_{\text {peak }}-\mathrm{v}_{\text {base }}\right) / \mathrm{v}_{\text {base }}}
$$




$$
\mathrm{mFMD}_{\mathrm{v}}=\frac{\left(D_{\text {peak }}-\mathrm{D}_{\text {base }}\right) / \mathrm{D}_{\text {base }}}{\left(\mathrm{v}_{\text {mean }}-\mathrm{v}_{\text {base }}\right) / \mathrm{v}_{\text {base }}}
$$

As glucose loading has been consistently shown to reduce brachial artery \%FMD in healthy individuals ${ }^{19-21,24-26}$, we evaluated the tracking performance of $\mathrm{pFMD}_{\mathrm{v}}$ and $\mathrm{mFMD}_{\mathrm{v}}$ in comparison with existing FMD indices ${ }^{7,11,14,34-36}$ in 22 presumed healthy male volunteers undergoing a standard oral glucose tolerance test, at two occasions. For the $\mathrm{FMD}_{\mathrm{v}}$ measures we established the reproducibility and comparatively evaluated their ability to detect acute changes in brachial artery flow-mediated dilation after oral glucose loading.

A

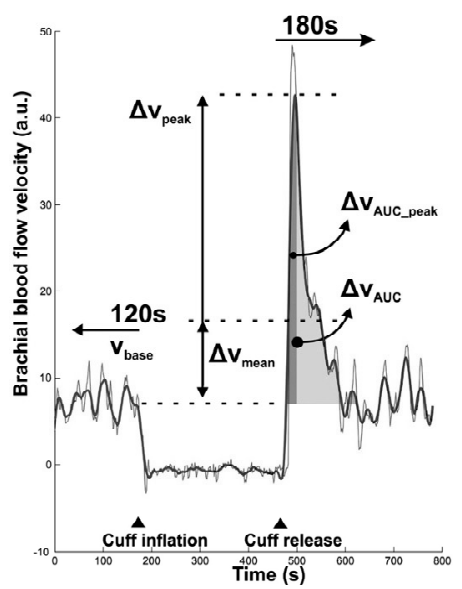

B FMD response

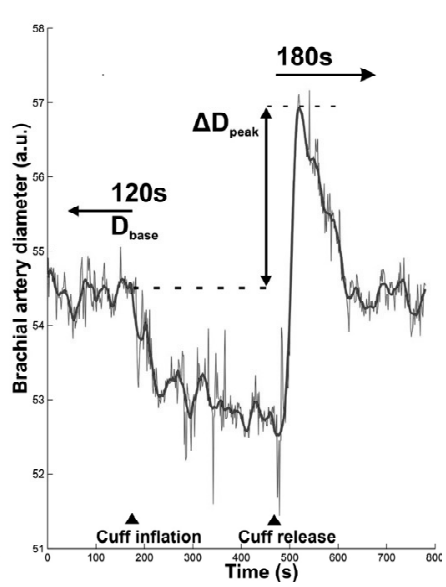

Figure 3.1 Definitions of baseline and hyperaemic brachial artery blood flow velocity and diameter parameters for the assessment of flow-mediated dilation. Blood flow velocity (A) and diameter curves (B) obtained by beat-to-beat analysis of Duplex video images. Raw data curves were smoothed to reduce noise, particularly in the peak change estimates. Baseline blood flow velocity ( $\left.v_{\text {base }}\right)$ and diameter $\left(D_{\text {base }}\right)$ were determined over the 120 seconds prior to cuff-inflation. The peak change in blood flow velocity $\left(\Delta \mathrm{v}_{\text {peak }}\right)$ was determined as the maximum velocity reached within 180 seconds after cuff-release, while the mean change in blood flow velocity $\left(\Delta v_{\text {mean }}\right)$ was determined as the average velocity over the 180 seconds after cuff-release, both minus the baseline blood flow velocity $v_{\text {base }}(\mathbf{A})$. The peak change in diameter $\left(\Delta D_{\text {peak }}\right)$ was determined as the maximum diameter reached within 180 seconds after cuff-release minus the baseline diameter $D_{\text {base }}(B)$. Indicated also are the full area under the curve of the hyperaemic velocity curve above baseline level $\left(\Delta \mathrm{v}_{\mathrm{AUC}}\right)$ and the area till peaktime of velocity curve $\left(\Delta \mathrm{v}_{\mathrm{AUC} \_ \text {peak }}\right)$. $\boldsymbol{\Delta}$ indicates the timing of rapid cuff inflation and release. 


\section{Materials and methods}

\section{Study population}

Twenty-two presumed healthy men (age range 18-46 years) participated. None had a history of diabetes, myocardial infarction or cardiovascular disease, or used medication. Height and weight were measured and body mass index (BMI, in $\mathrm{kg} / \mathrm{m}^{2}$ ) was calculated as the ratio of weight over height squared. Smoking status was determined by a questionnaire and classified as none, occasionally, or daily smoking. Pre-inclusion, fasting glucose was determined (OneTouch ${ }^{\circledR}$ Ultrasmart $^{\circledR}$, LifeScan, California, USA); each participant complied with the study limit, set at $\leq 6.1 \mathrm{mmol} / \mathrm{l}$. Blood pressure was measured with an automated oscillometric device with the cuff positioned around the left upper arm (Omron 705 IT, Omron Healthcare Europe B.V., Hoofddorp, the Netherlands).

The study was approved by the medical ethics committee of the Maastricht University Medical Centre (MUMC) and all participants gave written informed consent prior to enrolment.

\section{Study protocol}

Participants were asked to refrain from exercise, smoking, vitamin supplement use, and consumption of caffeine, alcohol, liquid (except water until 3 hours prior the start of measurements) or food twelve hours prior to the start of measurements. These were performed in the morning, with participants in supine position, in a quiet, climate-controlled room $\left(22-23^{\circ} \mathrm{C}\right)$ with dimmed lights, after a 15 minute period of acclimatization. A cannula was inserted into the left antecubital vein for blood sampling.

Participants underwent twice a set of measurements (spaced $8.5 \pm 3.4$ days apart) including an oral glucose tolerance test (OGTT; $82.5 \mathrm{~g}$ dextrose monohydrate in $250 \mathrm{ml}$ water plus additional $50 \mathrm{ml}$ water) at the University's Research Unit (Figure 3.2A). In each session, four flow-mediated dilation assessments were performed: two repeated measurements spaced ten minutes apart prior to glucose intake and two follow-up measurements after 30 and 75 minutes, respectively (Figure 3.2A).

\section{Flow-mediated dilation measurement}

The right shoulder and arm of the subjects were positioned on soft supports for optimal comfort and stability, to avoid muscle tension build-up and subsequent movement. Upper arm and wrist were rested on soft supports to avoid positioning artifacts due to cuff-inflation. A well-accessible segment of the right brachial artery was interrogated using a $7.5 \mathrm{MHz}, 40 \mathrm{~mm}$ linear-array ultrasound system (MyLab ${ }^{\mathrm{TM}} 70$, 
Esaote, Maastricht, the Netherlands) in Duplex-mode to record simultaneously artery diameter and centre-line blood flow sonogram. Emitted radiofrequency was set for highest resolution. The transducer position was fixed using a stereotactic probe holder, which provided all degrees of freedom to orient the probe at insonation angles perpendicular to the vessel long axis. The electronically steered pulsed-Doppler beam was set at an angle of 65-70 degrees with respect to the vessel long axis/flow direction. The length of the pulsed-Doppler sample volume was set at approximately half the vessel diameter to ensure consistent registration of the centre-line velocity despite possible movements over the recording period. Readjustment of the sample volume was avoided as much as possible because of the associated Doppler artifacts. A sphygmomanometer cuff (Hokanson TD 312, D.E. Hokanson, Inc., Bellevue, USA) was positioned on the right lower arm and inflated to $200 \mathrm{mmHg}$ for 5 minutes to elicit upon release the hyperaemic response required for brachial artery flowmediated dilation measurement (Figure 3.1B), as recommended. ${ }^{6-8}$ The cuff position was marked to ensure repeatable cuff repositioning within each session. For each flow-mediated dilation measurement, duplex images were recorded continuously on DVD over a total of 13 minutes: 3 minutes baseline (before rapid cuff-inflation), 5 minutes during lower arm cuff-inflation, and 5 minutes during hyperaemia following rapid cuff-release. A lead II ECG was recorded concurrently with the Doppler velocity signal to facilitate beat-by-beat measurements of brachial artery diameter and blood flow velocity in off-line video analysis.

\section{Duplex video analysis}

Recorded duplex video images were analyzed off-line using proprietary wall detection and Doppler velocity tracing software (Department of Biomedical Engineering, University of Maastricht, the Netherlands) implemented in Matlab (The Mathworks, Natick, MA). (A) Processing started with manual identification of the region of interest (ROI) within the B-mode image, containing a straight section of the artery. (B) Subsequently, the approximate position of the vessel wall-lumen interface was manually identified for both the anterior and posterior wall at the left and right side of the ROI. The lines through these points served as reference for the search region $(+/-1.5 \mathrm{~mm})$ for the wall-lumen interface in subsequent images. (C) The video frame coinciding with an ECG R-wave (i.e. end-diastolic) was automatically identified, utilizing the intensity coding of the ECG-waveform in the echo image. (D) For those images, first the search region was explored for the local echo maximum, reflecting the media-adventitia interface. The instantaneous wall position was identified starting from within the lumen as the first level-crossing (65\%) relative to the adventitia level $(100 \%)$, and provided an updated reference for the search region. The distance between the anterior and posterior wall positions averaged over the ROI was the estimate for the instantaneous diameter. (E) The instantaneous mean blood flow velocity was based on the spectral mean of the intensity distribution of the sonogram 
for each time instance, taking into account positive as well as negative flow-velocities (that typically occur in early diastole under baseline conditions). Its average over the sonogram, covering typically 3-5 heart cycles, was used for further processing. (F) The velocity and diameter samples were resampled at $1 \mathrm{~Hz}$ and low-pass filtered by a third order zero phase Savitsky-Golay filter with a width of 17 and 35 seconds, respectively, resulting in recordings as illustrated in Figures 3.1A (velocity) and $1 \mathrm{~B}$ (diameter), respectively. (G) Subsequently, the start and end of cuff-occlusion were manually identified, facilitating automatic extraction of the following parameters: (i) the baseline blood flow velocity $\left(v_{\text {base }}\right)$ and the baseline diameter $\left(D_{\text {base }}\right)$ are medians over the 120 seconds prior to cuff-inflation; (ii) the absolute peak change in blood flow velocity $\left(\Delta \mathrm{v}_{\text {peak }}\right)$ and the absolute peak change in diameter $\left(\Delta \mathrm{D}_{\text {peak }}\right)$ are the peaks of the velocity and diameter curves within the first 180 seconds after cuff-release relative to their baseline values (Figure 3.1A and 3.1B); (iii) the mean change in velocity $\left(\Delta v_{\text {mean }}\right)$ is the average of the curve for the same period minus the baseline value, and (iv) the relative peak change in blood flow velocity, the relative mean change in blood flow velocity and relative peak change in diameter are calculated as $\Delta v_{\text {peak }} / v_{\text {base, }} \Delta v_{\text {mean }} / v_{\text {base }}$ and $\Delta D_{\text {peak }} / D_{\text {base, }}$, respectively. $F M D_{v}$ indices were calculated as given in the introduction.

A
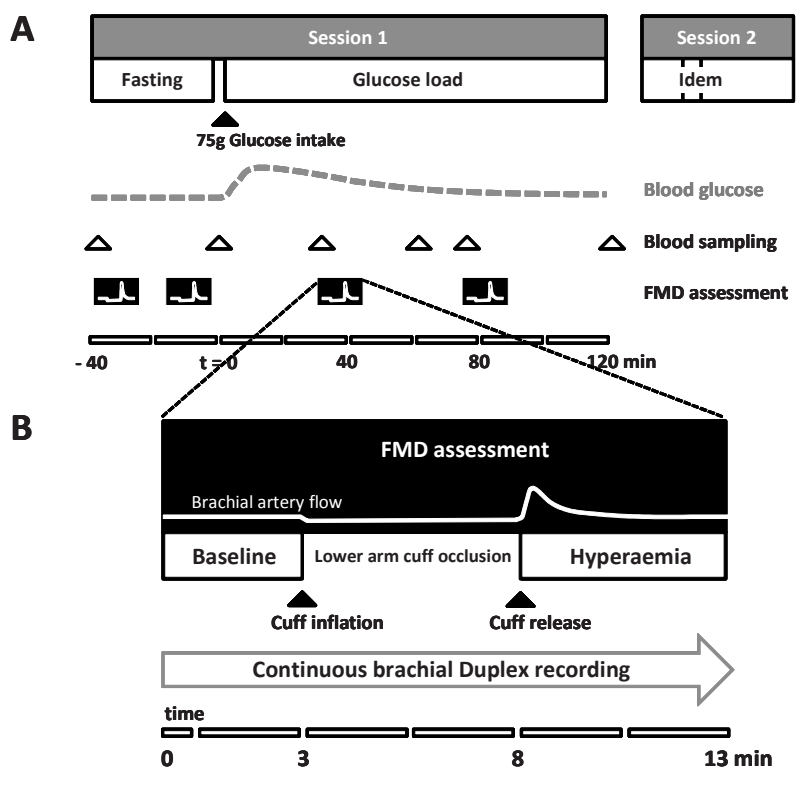

Figure 3.2 Detailed timelines of $(\mathbf{A})$ the study protocol and (B) the flow-mediated dilation assessment. (A) Changes in brachial artery flow-mediated dilation after glucose loading were assessed repeatedly, sessions 1 and 2 about one week apart. (B) A 5-minute lower arm cuff-occlusion was used to elicit a hyperaemic response to measure flow-mediated dilation from a continuous brachial Duplex ultrasound recording (B-mode and pulsed Doppler). Note the stimulus-response type of assessments for both (A) and (B) and the correspondingly different timescales. 
The automated determination of abovementioned values was verified by visual inspection of the recordings (as shown in Figure 3.1A and 3.1B), since (movement) artifacts and baseline drift may render automatic quantification invalid. ${ }^{6-8}$ Verification was performed blinded for the session and time-point of the flow-mediated dilation measurement, in random order, and prior to further data-analysis. As a result of this blinded procedure, $3 \%$ of the total 176 recordings were fully excluded. Of the included measurements, $10 \%$ of baseline diameter, $3 \%$ of peak diameter and $44 \%$ of baseline blood flow velocity values were manually corrected.

To compare the $\mathrm{FMD}_{\mathrm{v}}$ approach with existing indices that normalize the dilatory response to stimulus strength we calculated the relative peak diameter increase-tohyperaemic area under the curve (AUC) of blood flow velocity, obtained by integration (i) from cuff-release till peak velocity ${ }^{11}$ and (ii) over de full response (till 180 seconds $)^{36}:\left(\Delta D_{\text {peak }} / D_{\text {base }}\right) / \Delta v_{\text {AUc_peak }}$ and $\left(\Delta D_{\text {peak }} / D_{\text {base }}\right) / \Delta v_{A U c}$, respectively (see also Figure 3.1 for definitions). The corresponding indices originally normalize to shear rate and hence need to take into account blood viscosity and a parabolic velocity profile to estimate shear rate from mean or centre-line velocity. However, our focus is on tracking ability within individuals and short-term, i.e. relative changes with glucoseloading, which makes these factors irrelevant for our comparative analysis.

\section{Blood sample processing}

All blood samples were centrifuged at $3000 \mathrm{rpm}$ for $15 \mathrm{~min}$ and plasma was stored in $-80^{\circ} \mathrm{C}$ freezers until further analysis. The glucose concentration was determined by standardized glucose oxidase method (Synchron LX20 pro, Beckman Coulter Inc. CA, USA), with intra- and inter-assay coefficients of variation of 2.0 and $3.0 \%$, respectively.

\section{Statistical analysis}

Data are reported as means \pm SD. Reliability and test-retest agreement estimates were calculated on data of the first and second flow-mediated dilation assessments of both sessions (i.e. all fasting flow-mediated dilation assessments). ${ }^{37}$ To assess the reliability of the flow-mediated dilation parameters, intra-session and inter-session intra-class correlation coefficients (ICC) and coefficients of variation (CV=100\%.SD/ mean) were calculated using ANOVA. ${ }^{37,38}$ Bland-Altman plots of the differences between the data of the first and second session (inter-session) versus their mean were used to evaluate the limits of agreement between sessions. ${ }^{39}$

Generalized estimating equations (GEE) were used to compare the repeated glucose concentrations, diameter parameters (including \%FMD), blood flow velocity parameters, and both proposed $\mathrm{FMD}_{\mathrm{v}}$ indices before and after glucose intake. GEE are suitable for longitudinal data analyses and take into account the correlation between repeated measurements within individuals over time. ${ }^{40}$ Moreover, they have the 
advantage of robustly handling missing data and allow modeling of unequally-spaced time intervals. In the present study, the GEE models thus included session, time and interaction terms between session and time, the latter of which enabled to investigate whether both sessions showed similar results at corresponding time-points. ${ }^{40}$ Time (treated as a dummy variable with the fasting time-points as reference categories) was considered the independent variable and the repeated glucose concentrations, diameter and blood flow velocity parameters, and the $\mathrm{FMD}_{\mathrm{v}}$ and other indices as dependent variables (i.e. eight flow-mediated dilation assessments, four in each session). In these analyses an exchangeable correlation structure was used. ${ }^{40}$ ICCs were obtained with STATA software package version 9.2 (STATA Corp., Texas, USA) and GEE were analyzed with SPSS (Statistical Package for Social Sciences, version 20, IBM Corp., USA). A two-sided p-value $<0.05$ was considered statistically significant.

\section{Results}

The 22 participants were $23.5 \pm 5.8$ years of age (mean $\pm S D$ ), had a normal body mass index $\left(23.2 \pm 3.2 \mathrm{~kg} / \mathrm{m}^{2}\right.$ ), and were normotensive (systolic blood pressure $119 \pm 8$ $\mathrm{mmHg}$; diastolic blood pressure $67 \pm 4 \mathrm{mmHg}$ ), normoglycaemic (fasting glucose $4.5 \pm 0.3 \mathrm{mmol} / \mathrm{l}$ ) and non-smokers (none $\mathrm{n}=20$, occasional $\mathrm{n}=2$, daily $\mathrm{n}=0$ ). Blood glucose concentrations, brachial artery diameter and blood flow velocity derived parameters, \%FMD and both $\mathrm{FMD}_{\mathrm{v}}$ indices are presented for each time-point and for each session in Table 3.1. None of the variables did significantly differ between sessions at any corresponding time-point (all p-values $\geq 0.071$ ).

\section{Reproducibility}

The intra- and inter-session CVs for the baseline diameter were 2.4 and 3.5\%, for the relative peak change in diameter (\%FMD) 23 and 32\%, for the baseline blood flow velocity 27 and 28\%, for the relative peak change in blood flow velocity 28 and 29\%, and for the relative mean change in blood flow velocity 38 and $36 \%$, respectively. The intra- and inter-session CVs for $\mathrm{PFMD}_{v}$ were 43 and $45 \%$ and for $\mathrm{mFMD}_{v} 51$ and 51\%, respectively.

Inter-session ICCs for the $\mathrm{FMD}_{\mathrm{v}}$ indices were comparable with those of \%FMD (within 0.526-0.565), despite a somewhat lower ICC for the $\Delta \mathrm{v}_{\text {mean }} / \mathrm{v}_{\text {base }}$ component of the $\mathrm{mFMD}_{\mathrm{v}}$ (Table 3.1). Compared to the inter-session ICCs, the intra-session ICCs were somewhat higher for the diameter parameters and \%FMD but similar for the velocity parameters, $\mathrm{pFMD}_{\mathrm{v}}$ and $\mathrm{mFMD}_{\mathrm{v}}$ (Table 3.1). Bland-Altman plots indicated no dependency of differences between inter-session data with their mean for any of the flow-mediated dilation parameters (data not shown). Inter-session bias was very low ( $<7 \%)$ but variability was substantial: mean difference (limits of agreement) for \%FMD of $0.02 \%(-4.68 \% ; 4.72 \%)$ (mean value of $5.8 \%)$, for $\mathrm{pFMD}_{\mathrm{v}}$ of $0.001(-0.013 ; 0.015)$ (mean value of 0.015$)$, and for $\mathrm{mFMD}_{v}$ of $0.002(-0.067 ; 0.071)$ (mean value of 0.070$)$. 


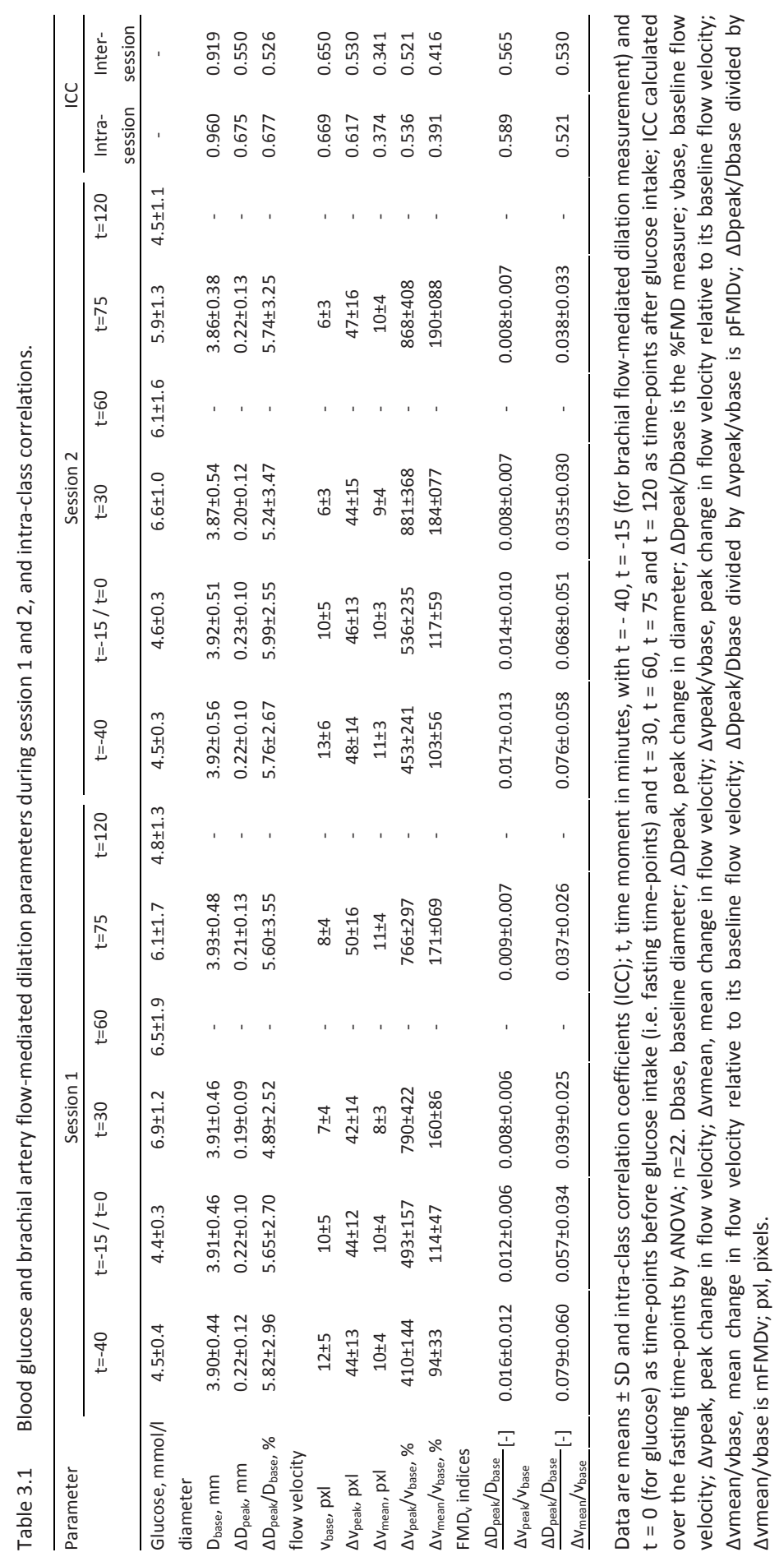




\section{Blood glucose}

Blood glucose concentration rose from $4.5 \mathrm{mmol} / \mathrm{l}$ at baseline (no difference between $-40 \mathrm{~min}$ and $0 \mathrm{~min} ; \mathrm{p}=0.88)$ to $6.8 \mathrm{mmol} / \mathrm{l}$ at $30 \mathrm{~min}(\mathrm{p}<0.001)$ after glucose intake, and gradually returned to $4.6 \mathrm{mmol} / \mathrm{l}$ at $120 \mathrm{~min}$ [ $<<0.001$, compared to $30 \mathrm{~min}$ level (Table 3.1)]. Brachial mean arterial pressure did not change with glucose loading $(p>0.23)$.

\section{Change in \%FMD with glucose loading}

The baseline diameter of each flow-mediated dilation assessment remained constant throughout most of the experiment (Figure 3.3A), but did show a significant decrease $30 \mathrm{~min}$ after glucose intake compared to the first fasting measurement at $-40 \mathrm{~min}$ $(p=0.044)$. Compared to fasting (either at $-40 \mathrm{~min}$ or at $-15 \mathrm{~min})$, at $30 \mathrm{~min}$ after glucose intake the absolute peak change in diameter was decreased, but it was near normal again after $75 \mathrm{~min}$ (Figure 3.3B). Similarly, \%FMD tended to be reduced $30 \mathrm{~min}$ after glucose intake (about 11\% lower, Table 3.2), but did not differ from the fasting value at $75 \mathrm{~min}$ post glucose (Figure 3.3C).
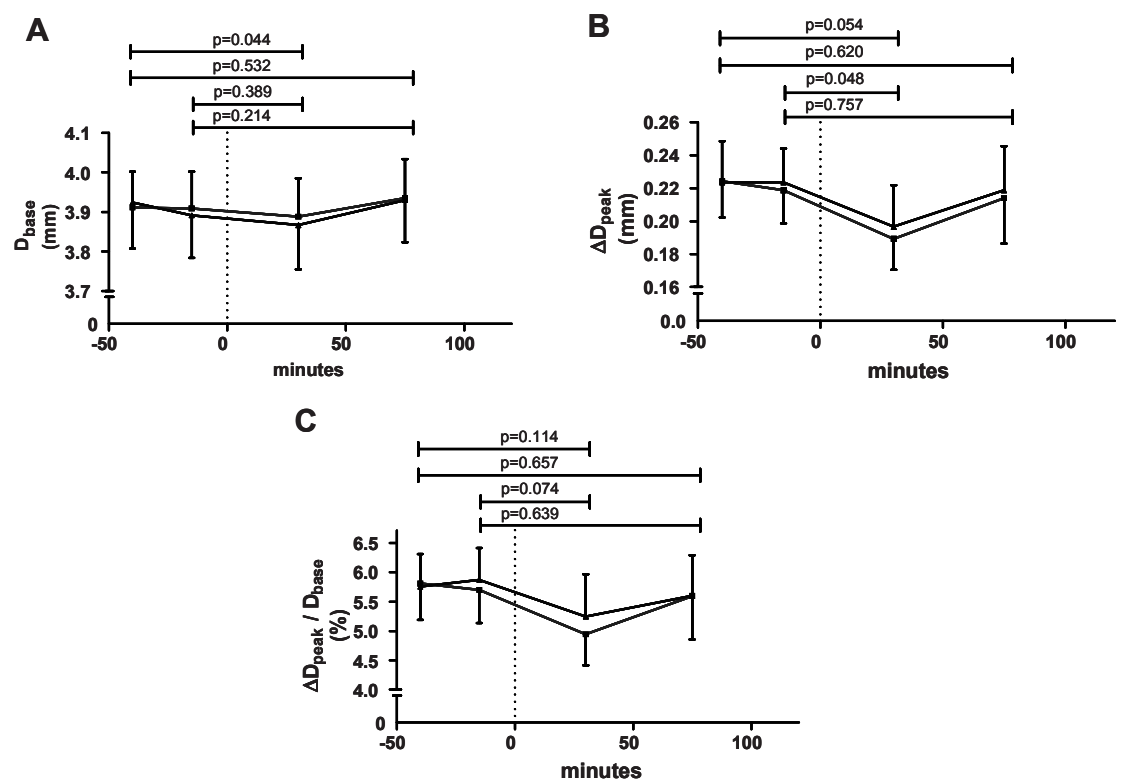

Figure 3.3 Changes in (A) baseline diameter and (B) the absolute and (C) the relative (i.e. \%FMD) peak changes in brachial artery diameter after glucose loading, as observed during flow-mediated dilation assessments at the 4 time-points indicated. Glucose was ingested at time-point 0 minutes (dashed vertical line). Gray line represents session 1 and black line session 2; whiskers indicate standard error. Data did not differ between sessions at any corresponding time-point (all $p$-values $\geq 0.568, n=22$ ). The $p$-values on top of the graphs refer to differences between pre and post glucose intake (of the mean of both sessions). Abbreviations as defined in Table 3.1. 


\section{Change in blood flow velocity with glucose loading}

Compared to fasting, baseline blood flow velocity was consistently lower after glucose intake ( $p<0.001$, Figure $3.4 \mathrm{~A}$ ). The absolute peak hyperaemic change in blood flow velocity did not change after glucose intake ( $p \geq 0.123$, Figure 3.4B). The absolute mean change in blood flow velocity showed a similar pattern, but was significantly lower at $+30 \mathrm{~min}$ ( $\mathrm{p} \leq 0.019$, Figure $3.4 \mathrm{C}$ ). Compared to fasting, both $\Delta \mathrm{v}_{\text {peak }} / \mathrm{v}_{\text {base }}$ and $\Delta \mathrm{v}_{\text {mean }} / \mathrm{v}_{\text {base }}$ were significantly $(p<0.001)$ and consistently higher after glucose intake (Figure 3.4D and 3.4E).
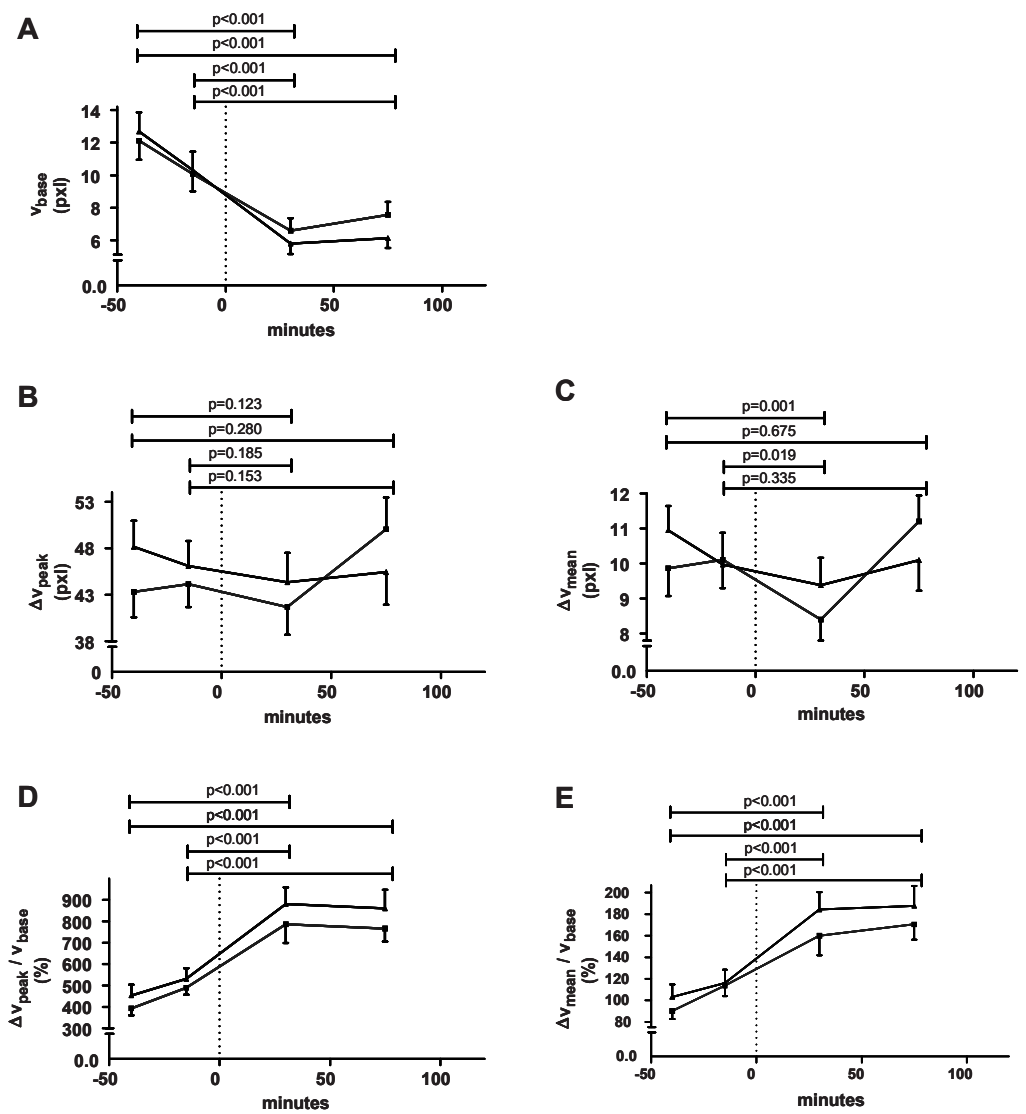

Figure 3.4 Changes in the baseline blood flow velocity (A), and the absolute changes in peak (B) and mean (C) blood flow velocity and relative changes in peak (D) and mean (E) blood flow velocity after glucose loading, as observed during flow-mediated dilation assessments at the 4 time-points indicated. Glucose was ingested at time-point 0 minutes (dashed vertical line). Gray line: session 1, black line: session 2; whiskers indicate standard error. Data did not differ between sessions at any corresponding time-point (all $p$-values $\geq 0.071, n=22$ ). The $p$-values on top of the graphs refer to differences between pre and post glucose intake (of the mean of both sessions). Abbreviations as defined in Table 3.1. 


\section{Change in $\mathrm{pFMD}_{\mathrm{v}}$ and $\mathrm{mFMD}_{\mathrm{v}}$ with glucose loading}

Compared to fasting, both $\mathrm{pFMD}_{\mathrm{v}}$ and $\mathrm{mFMD}_{\mathrm{v}}$ were significantly ( $\mathrm{p} \leq 0.001$, Figure $3.5 \mathrm{~A}$ and $3.5 \mathrm{~B}$ ) and consistently reduced after glucose intake (about 45\% lower; Table 3.2).

A

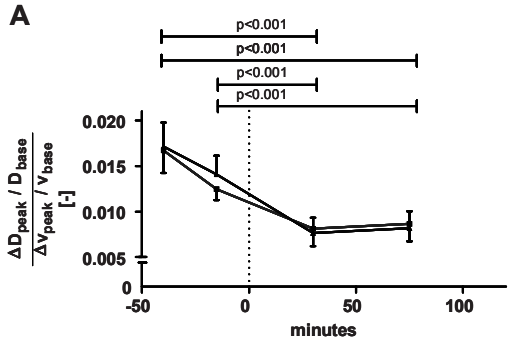

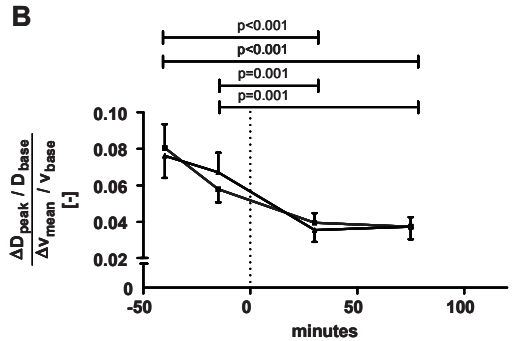

Figure 3.5 Changes in the relative diameter change normalized for (A) the relative peak change (pFMDv) and $(\mathbf{B})$ the relative mean change (mFMDv) in velocity after glucose loading. Glucose was ingested at time-point 0 minutes (dashed vertical line). Gray line: session 1, black line: session 2; whiskers indicate standard error. Data did not differ between sessions at any corresponding time-point (all $p$-values $\geq 0.176, n=22$ ). The $p$-values on top of the graphs refer to differences between pre and post glucose intake (of the mean of both sessions). Abbreviations as defined in Table 3.1.

\section{Additional analyses}

None of the parameters (Table 3.1) differed between the repeated fasting assessments (all $p$-values $\geq 0.31$ ) except for $v_{\text {base }}(p=0.001)$, for $\Delta v_{\text {peak }} / v_{\text {base }}(p=0.006)$, for $\mathrm{pFMD}_{v}(p=0.013)$, and borderline significantly for $\Delta \mathrm{v}_{\text {mean }} / \mathrm{v}_{\text {base }}(p=0.066)$ and $\mathrm{mFMD}_{\mathrm{v}}(\mathrm{p}=0.078)$.

To ascertain whether the above results for the $\mathrm{FMD}_{\mathrm{v}}$ indices are not merely explained by their components, we additionally evaluated the following absolute responsestimulus ratios $\Delta \mathrm{D}_{\text {peak }} / \Delta \mathrm{v}_{\text {peak }}$ and $\Delta \mathrm{D}_{\text {peak }} / \Delta \mathrm{v}_{\text {mean }}$, and $\% \mathrm{FMD} / 100$ normalized to peak and mean hyperaemic blood flow velocity increase: $\left(\Delta D_{\text {peak }} / D_{\text {base }}\right) / \Delta v_{\text {peak }}$ and $\left(\Delta D_{\text {peak }} / D_{\text {base }}\right) / \Delta v_{\text {mean }}$. These indices showed average reductions of less than $5 \%$ after glucose intake and none of these were statistically significant (all p-values $\geq 0.6$; Table 3.2).

The indices normalizing the dilatory response to the area under the curve (AUC) of shear rate (reflected by relative changes of blood flow velocity with glucose; on top of baseline) showed greater reductions than $\% F M D$ and $D_{\text {peak }} / D_{\text {base, }}$ but smaller reductions than both $\mathrm{FMD}_{\mathrm{v}}$ indices (Table 3.2). AUC integration till peak velocity time (as for $\mathrm{FMD} /$ shear $_{\text {AUC_peak }}$ ) appeared proportionally effective in capturing changes in stimulus with glucose, while integration over the full hyperaemic period (as for 
$\mathrm{FMD} /$ shear $_{\mathrm{AUC}}$ ) enabled better detection of changes in local brachial artery flow mediated dilation (Table 3.2).

Times to peak hyperaemic velocity or to peak dilatory response did not change with glucose (all $p>0.1$ ). The mean difference between the velocity and diameter peak times was $39 \pm 23$ seconds, clearly showing an integrator-like response from the system (as illustrated by the measurement example used in Figure 3.1.)

Table 3.2 Tracking performance of $\mathrm{FMD}_{\mathrm{v}}$ compared to existing FMD indices.

\begin{tabular}{|c|c|c|c|c|}
\hline FMD index & $\begin{array}{l}\text { Calculation } \\
\text { (from present study data) }\end{array}$ & Reference & Reduction & $\mathrm{p}$-value \\
\hline$\Delta D_{\text {peak }}$ & $=D_{\text {peak }}-D_{\text {base }}$ & - & $11 \%$ & $\geq 0.048$ \\
\hline$\% F M D$ & $=\left(D_{\text {peak }}-D_{\text {base }}\right) / D_{\text {base }} * 100$ & Celermajer et al. ${ }^{8}$ & $11 \%$ & $\geq 0.074$ \\
\hline $\mathrm{pFMD}_{\mathrm{v}}$ & $\begin{aligned}= & \left(D_{\text {peak }}-D_{\text {base }}\right) / D_{\text {base }} \\
& /\left(v_{\text {peak }}-v_{\text {base }}\right) / v_{\text {base }}\end{aligned}$ & - & $47 \%$ & $<0.001$ \\
\hline $\mathrm{mFMD}_{\mathrm{v}}$ & $\begin{aligned}= & \left(D_{\text {peak }}-D_{\text {base }}\right) / D_{\text {base }} \\
& /\left(v_{\text {mean }}-v_{\text {base }}\right) / v_{\text {base }}\end{aligned}$ & - & $44 \%$ & $\leq 0.001$ \\
\hline$D_{\text {peak }} / D_{\text {base }}$ & idem & Atkinson et al. $^{2}$ & $11 \%$ & $\geq 0.074$ \\
\hline FMD/shear ${ }_{\text {AUC_peak }}$ & $\begin{aligned}= & \left(D_{\text {peak }}-D_{\text {base }}\right) / D_{\text {base }} \\
& / \Delta v_{\text {AUC_peak }}\end{aligned}$ & Pyke et al. ${ }^{30}$ & $20 \%$ & $\geq 0.016$ \\
\hline FMD/shear AUC $_{\text {A }}$ & $\begin{aligned}= & \left(D_{\text {peak }}-D_{\text {base }}\right) / D_{\text {base }} \\
& / \Delta v_{\text {AUC }}\end{aligned}$ & $\begin{array}{l}\text { Pyke et al. } \\
\text { Padilla et al. }\end{array}$ & $38 \%$ & $\leq 0.038$ \\
\hline$\Delta D_{\text {peak }} / \Delta v_{\text {peak }}$ & $\begin{aligned}= & \left(D_{\text {peak }}-D_{\text {base }}\right) \\
& /\left(v_{\text {peak }}-v_{\text {base }}\right)\end{aligned}$ & - & $3 \%$ & $\geq 0.639$ \\
\hline$\Delta \mathrm{D}_{\text {peak }} / \Delta \mathrm{v}_{\text {mean }}$ & $\begin{aligned}= & \left(D_{\text {peak }}-D_{\text {base }}\right) \\
& /\left(v_{\text {mean }}-v_{\text {base }}\right)\end{aligned}$ & - & $3 \%$ & $\geq 0.710$ \\
\hline $\mathrm{FMD} / \Delta \mathrm{v}_{\text {peak }}$ & $\begin{aligned}= & \left(D_{\text {peak }}-D_{\text {base }}\right) / D_{\text {base }} \\
& /\left(v_{\text {peak }}-v_{\text {base }}\right)\end{aligned}$ & - & $4 \%$ & $\geq 0.811$ \\
\hline $\mathrm{FMD} / \Delta \mathrm{v}_{\text {mean }}$ & $\begin{aligned}= & \left(D_{\text {peak }}-D_{\text {base }}\right) / D_{\text {base }} \\
& /\left(v_{\text {mean }}-v_{\text {base }}\right)\end{aligned}$ & - & $0 \%$ & $\geq 0.712$ \\
\hline
\end{tabular}

Diameter and velocity parameters as defined in the text. Reduction percentages indicate the maximal reduction found after glucose intake (mostly $30 \mathrm{~min}$ after). Note that for the two indices normalizing for area under the curve (AUC) taking into account viscosity is not relevant in this evaluation of relative reductions with glucose; hence the calculations as specified are appropriate.

\section{Discussion}

To the best of our knowledge, the present study is the first to demonstrate the effect on the hyperaemic flow-stimulus with glucose loading in relation to its consequence for the quantitative assessment of changes in brachial artery flow-mediated dilation. We found that 1 ) the indices that take into account stimulus magnitude $\left(\mathrm{FMD}_{\mathrm{v}}\right.$ and $\mathrm{FMD} /$ shear $_{\mathrm{AUC}}$ ) show greater and significant reductions with glucose loading than the conventional \%FMD measure (not significant), 2) the reproducibility of the proposed $\mathrm{FMD}_{v}$ indices is similar to that of \%FMD, while 3) $\mathrm{FMD}_{v}$ improves the ability to detect acute changes in brachial artery flow-mediated dilation after oral glucose loading, also 
when compared to the other existing measures. Consideration of either the peak or the mean hyperaemic change in blood flow velocity in $\mathrm{FMD}_{\mathrm{v}}$ did not show obvious differences in performance.

\section{Reproducibility and reliability}

The present data indicate that the observed intra- and inter-session coefficients of variation for \%FMD and the $\mathrm{FMD}_{\mathrm{v}}$ indices are considerable. Since the reproducibility of the $\mathrm{FMD}_{v}$ indices and \%FMD, as assessed by ICCs and Bland-Altman analysis, was similar, we conclude that accounting for the (changes in) hyperaemic stimulus does not necessarily improve measurement reproducibility. However, our approach does improve the ability to discriminate changes in flow-mediated dilation, which is evident when effect size is considered in relation to measurement variability. The change in the $\mathrm{FMD}_{\mathrm{v}}$ indices after glucose intake compared to baseline, normalized to their intrasession CVs, was about 1 (for $\mathrm{pFMD}_{\mathrm{v}} 47 \% / 43 \%$ and for $\mathrm{mFMD}_{\mathrm{v}} 44 \% / 51 \%$ ), while for $\% F M D$ the ratio was only $0.52(11 \% / 23 \%)$. Agreement between sessions in terms of bias was good, with relative mean differences below $7 \%$ for the $\mathrm{FMD}_{\mathrm{v}}$ indices and \%FMD. For \%FMD this compares favourably with reported relative mean differences of $25 \%\left(1.6 / 6.5^{*} 100 \%\right)^{14}$ and $17 \%(1.1 / 6.5 * 100 \%) .{ }^{16}$ The lower value may be explained by the fact that we considered the average of two baseline flow-mediated dilation assessments per session. Furthermore, the observed $23 \%$ and $32 \%$ of intra- and intersession CVs for \%FMD fall within the previously reported range of $13-50 \% .{ }^{14-18}$

\section{Accounting for stimulus-response relationship in FMD assessment}

First, it should be emphasized that stimulus normalization (or baseline diameter normalization for that matter) in the present study has no intention of improving classification of subjects ${ }^{31,34,41}$, but rather is the direct consequence of considering the continuous physical interrelationship between local wall shear stress, vessel diameter and blood flow velocity. While for subject classification statistical adjustments seem more appropriate ${ }^{31,34,41}$, respecting the stimulus-response relation in tracking changes within individuals does imply considering the relative changes in flow velocity and diameter with hyperaemia (see introduction). Of note, the presumptions our approach includes are identical to existing and well-accepted approaches. . $^{3,6,11,30-32,35,36}$

The improved ability of the $\mathrm{FMD}_{\mathrm{v}}$ indices to detect the acute reduction in flowmediated dilation after glucose loading is explained by the direct relation between local blood flow velocity and vessel diameter. Both are continuous opposing influences that modulate wall shear stress. The present data demonstrate that when only diameter is considered, i.e. by using $\% \mathrm{FMD}^{35}$ or $\mathrm{D}_{\text {peak }} / \mathrm{D}_{\text {base }}{ }^{34}$ to quantify the effective action of brachial artery flow-mediated dilation in response to (distal) hyperaemia, the reduction is underestimated $(11 \%)$, while harder to detect (Table 3.2). By taking into account concomitant changes in the hyperaemic stimulus, 
the proposed $\mathrm{FMD}_{\mathrm{v}}$ indices more specifically and more robustly reflect the reduced ability of the brachial artery (about 45\%, Table 3.2) after glucose intake to locally normalize wall shear stress through vasodilating. The AUC-normalized indices did also improve detection, as expected from previous work of others. ${ }^{11,36}$ However, these measures appear less sensitive in tracking the acute reduction in flow-mediated dilation after glucose loading in our study (Table 3.2).

We investigated two possible parameters for the flow-stimulus; the relative peak blood flow velocity and the relative mean blood flow velocity. It is generally agreed upon that the time integral (i.e. mean blood flow velocity) should be considered to quantify the flow-stimulus responsible for dilatory response. ${ }^{11}$ The problem with this approach is to define a proper time window, because so far little has been elucidated about the time constants/delays of the various elements acting with flow-mediated dilation (mechano-sensing, signal transduction, phosphorylation, NO diffusion, muscle relaxation). Even if the time integral is properly restricted (e.g. by visual inspection), it does not provide a conclusive quantification of the effective flow-stimulus because of its transient nature. For a short stimulus, the peak of the flow-stimulus may probably be more relevant than the mean flow-stimulus via the time integral, but we did not find differences between both $\mathrm{FMD}_{\mathrm{v}}$ indices. In contrast, for the AUC-normalized indices the integrating nature of the system is apparent from the improvement in tracking ability when integrating beyond peak-velocity time (Table 3.2). The work of Padilla et al. and Pyke et al. shows that integration beyond 1-2 minutes practically covers the full AUC of blood velocity. ${ }^{11,36}$ Our 180-seconds integration includes the full AUC, but does not presume a specific temporal relationship between stimulus and response, as done by some. ${ }^{11}$

Taken together, our observations do suggest that the relative rather than the absolute change in blood flow velocity more accurately describes the relevant stimulus for flow-mediated dilation, while the absolute measures were not able to detect the changes after glucose loading (Table 3.2).

\section{Discrimination of potential glucose-effect on brachial artery FMD and periphery}

Previous studies in healthy individuals reported that glucose intake resulted in a relative reduction in \%FMD of $28 \%$ after 30 minutes ${ }^{21}$, of $\sim 18 \%{ }^{25}$ and of $\sim 65 \%{ }^{19}$ after 1 hour, and of $\sim 43 \%$ after 2 hours ${ }^{24}$, all of which were statistically significant. Other studies indicated relative reductions of $11 \%^{26}$ and of $44 \%{ }^{20}$ after 1 hour, which were not significant (like ours; $11 \%$ relative reduction \%FMD after 30 minutes, Figure 3.3 and Table 3.2). Some studies did not observe a change or observed an increase. ${ }^{22,23}$ Some of these studies suggested a role for increased lipid peroxidation or reactive oxygen species, which might reduce flow-mediated dilation. ${ }^{20,21,24}$ Other evidence suggests that increased dicarbonyl stress might play a role. ${ }^{42}$ Whether these 
mechanisms indeed reduce flow-mediated dilation response after glucose intake could be more thoroughly investigated with the control systems approach (FMDv) evaluated in this study.

The discrepancy between the sustained reduction 75 minutes after glucose intake signaled by FMDv and the tendency for \%FMD to return to baseline at this stage, suggests an impact on both the brachial artery flow-mediated dilation and peripheral vascular reactivity after glucose loading. A clear decrease in the absolute baseline flow-velocity was observed after glucose loading (Figure 3.4). The lower absolute baseline flow velocity might stem from an increased peripheral vascular resistance, considering that mean arterial pressure and brachial artery diameter did not change after glucose intake. The increased peripheral vascular resistance might be explained by glucose itself, which diminishes peripheral vascular diameter. ${ }^{43}$ However, it should be noted that the postprandial response of the peripheral vasculature is rather complex and only partially understood. ${ }^{44}$ With our approach the origin of changes in the hyperaemic flow-stimulus is not relevant for the quantification of brachial artery flow-mediated dilation. However, it is relevant to note that pharmacological and dietary interventions, or conditions such as obesity and diabetes ${ }^{9,44}$ are likely to influence or modulate the hyperaemic flow stimulus, requiring due consideration in assessing changes or differences in flow-mediated dilation. In the present study, the lower baseline value in blood flow velocity in combination with similar absolute hyperaemic changes converts to a relative increase in flow-stimulus after glucose loading (Figure 3.4), which is not accounted for by \%FMD, Dpeak/Dbase or the AUC of shear normalized indices. To fully appreciate the information incorporated in FMDv, we have summarized the measured steady-state and dynamic changes in local brachial flow velocity and diameter in Figure 3.6. FMDv specifically indicates how well local wall shear stress changes are normalized by brachial artery vasodilatory action. Interestingly, considering the intrinsically different approaches underlying FMDv and FMD/shearAUC in quantifying FMD performance, they both show a more distinct change with hyperglycaemia than the measures not accounting for stimulus strength (Table 3.2). We submit that FMDv is the most comprehensive physically valid means to assess acute changes in specifically brachial artery flow-mediated dilation performance, by properly accounting for (concomitant) changes in peripheral haemodynamics and vascular reactivity.

\section{Strengths and limitations}

The present study has strengths and limitations. Strengths include: (A) flow-mediated dilation measurements were executed according to the latest international recommendations. $^{5-8}$ (B) Extraction of the diameter and blood flow velocity parameters was semi-automatic and fully blinded. (C) The continuous assessment over 13 minutes $^{7}$ : (i) provided detailed blood flow velocity and diameter curves, 
excluding time ambiguity with respect to the derived blood flow velocity and diameter variables, and (ii) enabled visual inspection of the blood flow velocity and diameter curves for artifacts and/or drift, allowing verification of the automatically determined values. (D) The repeated execution of the protocol enables comprehensive assessment of reproducibility, which contributed to reliability of our observations. (E) In an additional series of measurements in $n=8$ control subjects with similar characteristics undergoing exactly the same protocol, but taking plain water instead of the glucose solution, we investigated whether changes in flow velocity (v), diameter (D) or FMD indices occurred as a result of the protocol rather than glucose intake.

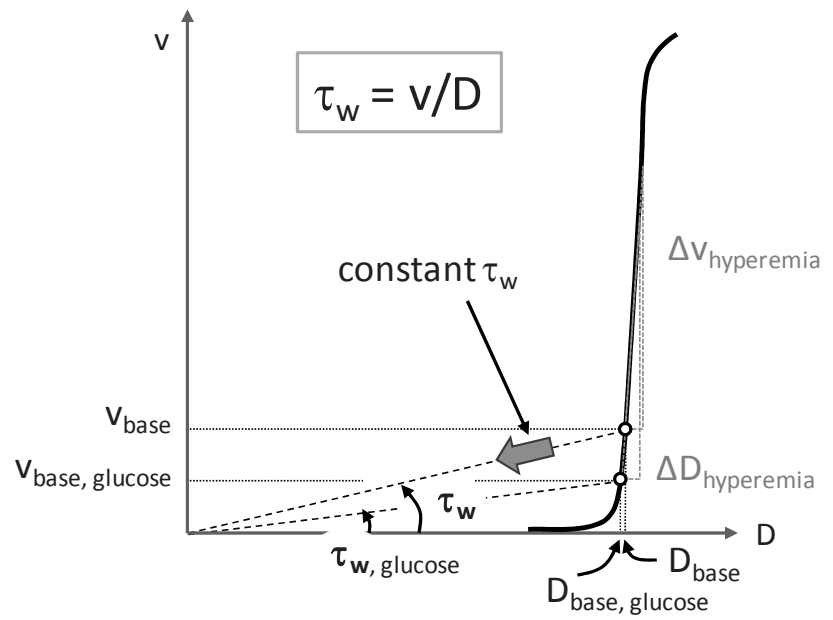

Figure 3.6 Interpretation of measured changes in local brachial artery flow velocity (v) and diameter (D) after glucose intake. Please note that scales are all conform the relative magnitudes of the effects observed in the study group. The steady-state working point of the wall shear stress $\left(\tau_{w}\right)$ normalization control loop apparently shifts down on the (assumed) function curve going from fasting to after glucose intake, while the corresponding hyperaemic changes remain relatively comparable. The ideal case of a full normalization of local brachial wall shear stress under the steady-state hyperglycaemic and hyperaemic conditions is indicated by the dashed lines from origin to working point ('constant $\left.\tau_{\mathrm{w}}{ }^{\prime}\right)$. As captured by our system parameter FMD, we found a clear deviation from the ideal line (i.e. proportionally changing $v$ and $D$ ) indicating wall shear stress normalization is normally far from ideal and (slightly) deteriorates under hyperglycaemic conditions.

In these controls we observed a significant decrease in $v_{\text {base }}$ of about $25 \%$ ( $n=8$, $p<0.01$; other parameters: $p>0.05$ ). It is important to note that our study, however, was not designed to test the hypothesis whether glucose itself, a volume load (both water and glucose solution) or repeated use of the FMD apparatus caused the changes in flow velocity (v), diameter (D) or FMD indices. Nevertheless, compared to the $45 \%$ decrease we found in the intervention group $(n=22, p<0.001)$ we may conclude that, at least in our study, repeated hyperaemic challenges did have an 
additional effect on assessment of FMD. If we assume that changes in $v_{\text {base }}$ in the water controls are representative of the non-glucose related changes in our glucose load subjects, then the glucose-specific effect may be conservatively expressed as roughly half the change in FMDv as reported in Table 3.2. This finding is in contrast to the findings of Harris et al. who reported no significant changes in \%FMD $(n=20)$ and hyperaemic volume flow (separate study in $n=6) .{ }^{45}$ However, their methodology, i.e. no Duplex recordings and a separate study in $n=6$ of volume flow (larger variability in estimate) instead of flow velocity, violates the experimental requirements to directly link changes in $v$ and $D$ within measurements and subjects, which we specifically aimed to do in our study. Nevertheless, the problems associated with hyperaemic stimulus creation for FMD assessment and the impact on (acute) intervention studies remains to be further investigated. We submit that our systems approach can and should be included in these future evaluations, using a routine ultrasound technique.

Limitations include: (a) the results were obtained in healthy men, limiting direct inferences to women or diseased individuals. (b) The FMDv indices are based on a steady state relationship between flow-stimulus and dilation response, which is not necessarily suited to transient hyperaemic flow-stimuli. ${ }^{8,30}$ (c) Separate measurements of the peripheral vasculature were not included, which limits firm conclusions with regard to the origin of the increased stimulus after glucose intake. (d) The present analysis cannot exclude a carry-over effect between first and second flow-mediated dilation assessments (spaced $10 \mathrm{~min}$ apart in each session) potentially caused by repeatedly engaging the flow-mediated dilation apparatus. Similarly, as discussed above, in control measurements $(n=8)$ we found a tendency for baseline flow velocity to decrease with time $(p<0.01)$, which appears to be caused by the repeated hyperaemic challenges as such or further acclimatization of the subjects, despite our approach following current guidelines for vascular measurements. However, our observation that the pFMDv showed a significant decrease in the second compared to the first fasting flow-mediated dilation assessment (Table 3.1) may support our main finding that the $\mathrm{FMD}_{\mathrm{v}}$ indices, in contrast to \%FMD, are able to detect, more specifically and with greater power, short-term changes in local brachial artery flowmediated dilation.

\section{Conclusions}

We conclude that a control systems approach to quantification of flow-mediated dilation improves the detection of changes in brachial artery dilation after an oral glucose loading, by apparently better capturing the vasodilatory response of the artery aimed to normalize local wall shear stress. The FMDv approach may be particularly useful in acute intervention studies aimed at discriminating effects on conduit artery flow-mediated dilation and those on the microcirculation. 


\section{References}

1. Davies PF, Spaan JA, Krams R. Shear stress biology of the endothelium. Ann Biomed Eng. 2005; 33:1714-1718.

2. Hilgers RH, Bergaya S, Schiffers PM, Meneton $P$, Boulanger CM, Henrion D, Levy BI, De Mey JG. Uterine artery structural and functional changes during pregnancy in tissue kallikrein-deficient mice. Arterioscler Thromb Vasc Biol. 2003; 23:1826-1832.

3. Reneman RS, Arts T, Hoeks AP. Wall shear stress--an important determinant of endothelial cell function and structure--in the arterial system in vivo. Discrepancies with theory. J Vasc Res. 2006; 43:251-269.

4. Brunner H, Cockcroft JR, Deanfield J, Donald A, Ferrannini E, Halcox J, Kiowski W, Luscher TF, Mancia G, Natali A, Oliver JJ, Pessina AC, Rizzoni D, Rossi GP, Salvetti A, Spieker LE, Taddei S, Webb DJ. Endothelial function and dysfunction. Part II: Association with cardiovascular risk factors and diseases. A statement by the Working Group on Endothelins and Endothelial Factors of the European Society of Hypertension. J Hypertens. 2005; 23:233-246.

5. Corretti MC, Anderson TJ, Benjamin EJ, Celermajer D, Charbonneau F, Creager MA, Deanfield J, Drexler H, Gerhard-Herman M, Herrington D, Vallance P, Vita J, Vogel R. Guidelines for the ultrasound assessment of endothelial-dependent flow-mediated vasodilation of the brachial artery: a report of the International Brachial Artery Reactivity Task Force. J Am Coll Cardiol. 2002; 39:257-265.

6. Thijssen DH, Black MA, Pyke KE, Padilla J, Atkinson G, Harris RA, Parker BA, Widlansky ME, Tschakovsky ME, Green DJ. Assessment of flow mediated dilation (FMD) in humans: a methodological and technical guideline. Am J Physiol Heart Circ Physiol 2011; 300:H2-12.

7. Black MA, Cable NT, Thijssen DH, Green DJ. Importance of measuring the time course of flowmediated dilatation in humans. Hypertension. 2008; 51:203-210.

8. Harris RA, Nishiyama SK, Wray DW, Richardson RS. Ultrasound assessment of flow-mediated dilation. Hypertension. 2010; 55:1075-1085.

9. Henry RM, Ferreira I, Kostense PJ, Dekker JM, Nijpels G, Heine RJ, Kamp O, Bouter LM, Stehouwer CD. Type 2 diabetes is associated with impaired endothelium-dependent, flow-mediated dilation, but impaired glucose metabolism is not; The Hoorn Study. Atherosclerosis. 2004; 174:49-56.

10. Mitchell GF, Parise H, Vita JA, Larson MG, Warner E, Keaney JF, Jr., Keyes MJ, Levy D, Vasan RS, Benjamin EJ. Local shear stress and brachial artery flow-mediated dilation: the Framingham Heart Study. Hypertension. 2004; 44:134-139.

11. Pyke KE, Tschakovsky ME. Peak vs. total reactive hyperemia: which determines the magnitude of flow-mediated dilation? J Appl Physiol. 2007; 102:1510-1519.

12. Green DJ, Jones H, Thijssen D, Cable NT, Atkinson G. Flow-mediated dilation and cardiovascular event prediction: does nitric oxide matter? Hypertension. 2011; 57:363-369.

13. Inaba Y, Chen JA, Bergmann SR. Prediction of future cardiovascular outcomes by flow-mediated vasodilatation of brachial artery: a meta-analysis. Int J Cardiovasc Imaging. 2010; 26:631-640.

14. Woodman RJ, Playford DA, Watts GF, Cheetham C, Reed C, Taylor RR, Puddey IB, Beilin LJ, Burke V, Mori TA, Green D. Improved analysis of brachial artery ultrasound using a novel edge-detection software system. J Appl Physiol. 2001; 91:929-937.

15. Donald AE, Halcox JP, Charakida M, Storry C, Wallace SM, Cole TJ, Friberg P, Deanfield JE. Methodological approaches to optimize reproducibility and power in clinical studies of flow-mediated dilation. J Am Coll Cardiol. 2008; 51:1959-1964.

16. Ghiadoni L, Faita F, Salvetti M, Cordiano C, Biggi A, Puato M, Di Monaco A, De Siati L, Volpe M, Ambrosio G, Gemignani V, Muiesan ML, Taddei S, Lanza GA, Cosentino F. Assessment of flowmediated dilation reproducibility: a nationwide multicenter study. J Hypertens. 2012;30:1399-405.

17. Sorensen KE, Celermajer DS, Spiegelhalter DJ, Georgakopoulos D, Robinson J, Thomas O, Deanfield JE. Non-invasive measurement of human endothelium dependent arterial responses: accuracy and reproducibility. Br Heart J. 1995; 74:247-253.

18. Welsch MA, Allen JD, Geaghan JP. Stability and reproducibility of brachial artery flow-mediated dilation. Med Sci Sports Exerc. 2002; 34:960-965. 
19. Akbari CM, Saouaf R, Barnhill DF, Newman PA, LoGerfo FW, Veves A. Endothelium-dependent vasodilatation is impaired in both microcirculation and macrocirculation during acute hyperglycemia. J Vasc Surg. 1998; 28:687-694.

20. Kawano H, Motoyama T, Hirashima O, Hirai N, Miyao Y, Sakamoto T, Kugiyama K, Ogawa H, Yasue H. Hyperglycemia rapidly suppresses flow-mediated endothelium-dependent vasodilation of brachial artery. J Am Coll Cardiol. 1999; 34:146-154.

21. Mah E, Noh SK, Ballard KD, Matos ME, Volek JS, Bruno RS. Postprandial hyperglycemia impairs vascular endothelial function in healthy men by inducing lipid peroxidation and increasing asymmetric dimethylarginine:arginine. J Nutr. 2011; 141:1961-1968.

22. Major-Pedersen A, Ihlemann N, Hermann TS, Christiansen B, Dominguez H, Kveiborg B, Nielsen DB, Svendsen OL, Kober L, Torp-Pedersen C. Effects of oral glucose load on endothelial function and on insulin and glucose fluctuations in healthy individuals. Exp Diabetes Res. 2008; 2008:672021

23. Siafarikas A, Watts K, Beye P, Jones TW, Davis EA, Green DJ. Lack of effect of oral glucose loading on conduit vessel endothelial function in healthy subjects. Clin Sci (Lond). 2004; 107:191-196.

24. Title LM, Cummings PM, Giddens K, Nassar BA. Oral glucose loading acutely attenuates endotheliumdependent vasodilation in healthy adults without diabetes: an effect prevented by vitamins $\mathrm{C}$ and $\mathrm{E}$. J Am Coll Cardiol. 2000; 36:2185-2191.

25. Watanabe K, Oba K, Suzuki T, Ouchi M, Suzuki K, Futami-Suda S, Sekimizu K, Yamamoto N, Nakano H. Oral glucose loading attenuates endothelial function in normal individual. Eur J Clin Invest. 2011; 41:465-473.

26. Xiang GD, Sun HL, Zhao LS, Hou J, Yue L, Xu L. The antioxidant alpha-lipoic acid improves endothelial dysfunction induced by acute hyperglycaemia during OGTT in impaired glucose tolerance. Clin Endocrinol (Oxf). 2008; 68:716-723.

27. Nicholls SJ, Lundman P, Harmer JA, Cutri B, Griffiths KA, Rye KA, Barter PJ, Celermajer DS. Consumption of saturated fat impairs the anti-inflammatory properties of high-density lipoproteins and endothelial function. J Am Coll Cardiol. 2006; 48:715-720.

28. Weseler AR, Ruijters EJ, Drittij-Reijnders MJ, Reesink KD, Haenen GR, Bast A. Pleiotropic benefit of monomeric and oligomeric flavanols on vascular health - a randomized controlled clinical pilot study. PLoS One 2011; 6:e28460.

29. Nishiyama SK, Walter Wray D, Berkstresser K, Ramaswamy M, Richardson RS. Limb-specific differences in flow-mediated dilation: the role of shear rate. J Appl Physiol. 2007; 103:843-851.

30. Stoner L, Sabatier MJ. Use of ultrasound for non-invasive assessment of flow-mediated dilation. J Atheroscler Thromb. 2012; 19:407-421.

31. Parker BA, Trehearn TL, Meendering JR. Pick your Poiseuille: normalizing the shear stimulus in studies of flow-mediated dilation. J Appl Physiol. 2009; 107:1357-1359.

32. Pyke KE, Dwyer EM, Tschakovsky ME. Impact of controlling shear rate on flow-mediated dilation responses in the brachial artery of humans. J Appl Physiol. 2004; 97:499-508.

33. Silber HA, Bluemke DA, Ouyang P, Du YP, Post WS, Lima JA. The relationship between vascular wall shear stress and flow-mediated dilation: endothelial function assessed by phase-contrast magnetic resonance angiography. J Am Coll Cardiol 2001; 38:1859-1865.

34. Atkinson G, Batterham AM, Thijssen DH, Green DJ. A new approach to improve the specificity of flowmediated dilation for indicating endothelial function in cardiovascular research. J Hypertens 2013; 31:287-291.

35. Celermajer DS, Sorensen KE, Gooch VM, Spiegelhalter DJ, Miller OI, Sullivan ID, Lloyd JK, Deanfield JE. Non-invasive detection of endothelial dysfunction in children and adults at risk of atherosclerosis. Lancet. 1992; 340:1111-1115.

36. Padilla J, Johnson BD, Newcomer SC, Wilhite DP, Mickleborough TD, Fly AD, Mather KJ, Wallace JP. Normalization of flow-mediated dilation to shear stress area under the curve eliminates the impact of variable hyperemic stimulus. Cardiovasc Ultrasound. 2008; 6:44.

37. de Vet HC, Terwee CB, Knol DL, Bouter LM. When to use agreement versus reliability measures. J Clin Epidemiol. 2006; 59:1033-1039.

38. Bland JM. How do I analyse observer variation studies? http://www-users.york.ac.uk/ mb55/ obshead.htm. [4 JUL, 2013] 2005.

39. Bland JM, Altman DG. Statistical methods for assessing agreement between two methods of clinical measurement. Lancet. 1986; 1:307-310. 
40. Twisk JWR. Applied Longitudinal Data Analysis for Epidemiology: A Practical Guide. Cambridge University, Cambridge, United Kingdom, 2003.

41. Thijssen DH, van Bemmel MM, Bullens LM, Dawson EA, Hopkins ND, Tinken TM, Black MA, Hopman MT, Cable NT, Green DJ. The impact of baseline diameter on flow-mediated dilation differs in young and older humans. Am J Physiol Heart Circ Physiol. 2008; 295:H1594-1598.

42. Beisswenger PJ, Howell SK, O'Dell RM, Wood ME, Touchette AD, Szwergold BS. Alpha-Dicarbonyls increase in the postprandial period and reflect the degree of hyperglycemia. Diabetes Care. 2001; 24:726-732.

43. Renaudin C, Michoud E, Rapin JR, Lagarde M, Wiernsperger N. Hyperglycaemia modifies the reaction of microvessels to insulin in rat skeletal muscle. Diabetologia. 1998; 41:26-33.

44. Jonk AM, Houben AJ, Schaper NC, de Leeuw PW, Serne EH, Smulders YM, Stehouwer CD. Meal-related increases in microvascular vasomotion are impaired in obese individuals: a potential mechanism in the pathogenesis of obesity-related insulin resistance. Diabetes Care. 2011; 34 Suppl 2:S342-348.

45. Harris RA, Padilla J, Rink LD, Wallace JP. Variability of flow-mediated dilation measurements with repetitive reactive hyperemia. Vasc Med. 2006; 11:1-6. 
Part II

Diet and endothelial dysfunction and low-grade inflammation 


\section{Chapter 4}

Fish consumption in healthy adults is associated with decreased circulating biomarkers of endothelial dysfunction and inflammation, during a 6-year follow-up

BCT van Bussel, RMA Henry, CG Schalkwijk, I Ferreira, EJM Feskens, MT Streppel, YM Smulders, JWR Twisk, CDA Stehouwer J Nutr 2011;141:1719-1725 Reprinted with permission of the publisher 


\section{Abstract}

A healthy diet rich in fish, fruit and vegetables, moderate in alcoholic beverages and low in dairy products, has been associated with lower circulating concentrations of biomarkers of endothelial dysfunction (ED) and low-grade inflammation (LGI). It is, however, unknown how consumption of these food groups affects ED and/or LGI over time.

We measured diet by computer-assisted crosscheck dietary history method at $36 \pm 0.63$ years of age $(n=301$, women=161). At 36 years and 42 years of age, we measured von Willebrand factor, soluble intercellular adhesion molecule 1 (sICAM-1), soluble endothelial selectin, soluble vascular cell adhesion molecule 1 and soluble thrombomodulin (circulating biomarkers of ED) and C-reactive protein, serum amyloid A, IL-6, IL-8, tumour necrosis factor $\alpha$ and SICAM-1 (circulating biomarkers of LGI). We investigated the associations between food groups and changes in combined biomarker Z-scores of ED and LGI [higher scores associated with greater risk of (incident) cardiovascular disease].

After adjustment for sex, energy intake, body mass index, physical activity, alcohol consumption, smoking behaviour and other food groups, fish consumption (per $100 \mathrm{~g} / \mathrm{wk}$ ), but none of the other food groups, was inversely associated with changes in $\operatorname{ED}[\beta(95 \% \mathrm{Cl}):-0.06(-$ $0.10 ;-0.02), p=0.003]$ and LGI [-0.05 (-0.09;-0.003), $p=0.036]$. Additionally, EPA+DHA intake was inversely associated with changes in $\operatorname{ED}[\beta(95 \% \mathrm{Cl}):-0.13(-0.19 ;-0.07), p \leq 0.001]$ and LGI $[-0.09$ ($0.16 ;-0.02), p=0.013$ ] and explained $83 \%$ and $40 \%$ of the association between fish and changes in ED and LGI.

In conclusion, fish consumption, but not fruit, vegetable, alcoholic beverage or dairy product consumption, was associated with decreased ED and LGI in healthy adults. 


\section{Introduction}

Observational studies have shown that higher concentrations of circulating biomarkers of endothelial dysfunction and low-grade inflammation are associated with incident cardiovascular disease (CVD).$^{1-5}$ Von Willebrand factor (vWf), soluble vascular cell adhesion molecule 1 (sVCAM-1), soluble endothelial selectin (sE-selectin), soluble thrombomodulin (STM) and soluble intercellular adhesion molecule 1 (sICAM-1) are all synthesized by endothelial cells ${ }^{6-8}$ and C-reactive protein (CRP), serum amyloid A (SAA), interleukin 6 (IL-6), interleukin 8 (IL-8), tumour necrosis factor $\alpha$ (TNF- $\alpha$ ) and sICAM-1 are involved in the inflammatory process. ${ }^{7}$ For these reasons, it is plausible to assume that higher circulating concentrations of these markers reflect dysfunction of the endothelium and more inflammatory activity, respectively.

Diet is perhaps the most modifiable CVD risk factor ${ }^{9}$ as it has been shown that diets rich in fish, fruit and vegetables, with moderate alcohol and low dairy product consumption, are associated with a reduced incidence of CVD. ${ }^{10-14}$ The reduction in CVD associated with such a diet and the fact that endothelial dysfunction and lowgrade inflammation play an important role in the aetiology of $\mathrm{CVD}^{7}$ suggest that these food groups may modify the development of CVD through an improvement of endothelial dysfunction and low-grade inflammation. ${ }^{15}$

Indeed, previous studies showed that a healthy diet, including high consumption of fish, fruit and vegetables, and with moderate alcohol intake, was associated with lower levels of circulating markers of endothelial dysfunction, such as sE-selectin ${ }^{16,17}$, sICAM-1 ${ }^{16,18}$ and SVCAM-1. ${ }^{16}$ Furthermore, such a diet was associated with lower levels of circulating markers of low-grade inflammation, such as CRP ${ }^{16-18}$, IL-6 ${ }^{16,18}$ and SICAM- $1^{16,18}$, whereas high-fat dairy product consumption was associated with higher levels of sICAM-1. ${ }^{18}$

However, these studies relied on cross-sectional data ${ }^{16-18}$, none reporting on food consumption in relation to changes in endothelial dysfunction and low-grade inflammation over time.

In view of these considerations, we hypothesized that a high consumption of fish, fruit and/or vegetables, a moderate consumption of alcohol and/or a low consumption of dairy products is associated with a decrease of endothelial dysfunction and low-grade inflammation.

Therefore, we examined the association between the consumption of fish, fruit, vegetables, alcoholic beverages and dairy products and changes in a panel of five circulating biomarkers of endothelial dysfunction ( $v W f$, sVCAM-1, sE-selectin, sTM and sICAM-1), and a panel of six circulating biomarkers of low-grade inflammation (CRP, SAA, IL-6, IL-8, TNF- $\alpha$ and sICAM-1) in a population-based cohort of apparently healthy adults, over a 6 -year follow-up period. 


\section{Methods}

\section{Study population}

The Amsterdam Growth and Health Longitudinal Study (AGAHLS) is a longitudinal cohort study ( $n=698$ ) that started in 1976 (details have been described elsewhere). ${ }^{19-21}$ Briefly, its initial goal was to study the natural development of the growth, health and lifestyle of adolescents [age in 1976 (mean \pm SD): $13.1 \pm 0.8$ years] and to investigate longitudinal relationships between biological and lifestyle variables. At each follow-up measurement, dietary intake and other lifestyle (smoking behaviour and daily physical activity), anthropometric (body height, weight and skinfolds), biological (blood pressure, serum lipoprotein levels and physical fitness) and psychological variables were assessed. ${ }^{19-21}$ At the 2000 follow-up examination, dietary intake was assessed and blood samples were collected in 373 participants. Circulating biomarkers of endothelial dysfunction and low-grade inflammation were measured in serum or plasma samples from 373 participants who attended the 2000 follow-up examination, and again in 301 participants of these 373 participants, 6 year later (2006 examination). The current study therefore consists of 301 participants (161 women) in whom full data on diet [year 2000 (mean age 36.5 \pm 0.6 years)] and circulating biomarkers [years 2000 and 2006 (mean age $42.5 \pm 0.6$ years)] were available.

The study was approved by the ethics committee of the VU University Medical Center and all participants gave their written informed consent.

\section{Dietary intake}

To assess habitual dietary intake a computer-assisted crosscheck dietary history method (DISHES) was used that asked participants about their habitual dietary intake over the previous month. ${ }^{22-25}$ For logistical reasons, a less comprehensive dietary assessment method was used in 2006. Therefore, we have limited this study to the comprehensive baseline dietary data only. DISHES was carried out by trained nutritionists by means of face-to-face interviews and uses models of cups, glasses, spoons, plates and bowls for the estimation of portion sizes. Based on the dietary data food groups were constructed and nutrient intake was calculated with the help of the Dutch food composition database from 2006 (please see Appendix 4). ${ }^{26}$

Associations with fish consumption were further explored on a nutrient level by investigating the nutrient compounds eicosapentaenoic acid (EPA) and docosapentaenoic acid (DHA). Alcohol consumption was analyzed as a food group (alcoholic beverages in $\mathrm{I} / \mathrm{wk}$, including beer, wine and spirits), a nutrient compound (ethanol in $\mathrm{g} / \mathrm{d}$ ) and a lifestyle covariate [categorized into none, low (<median; median=16.8 g/d) and high consumers ( $\geq$ median)]. 


\section{Assessment of endothelial dysfunction and low-grade inflammation}

Circulating biomarkers of endothelial dysfunction and low-grade inflammation were determined in blood samples of the participants in the 2000 and 2006 follow-up examinations. Serum biomarkers of endothelial dysfunction (sVCAM-1, sE-selectin, sTM, sICAM-1), and of low-grade inflammation (CRP, SAA, IL-6, IL-8, TNF- $\alpha$ and sICAM-1) were assessed by a multi-array detection system based on electrochemiluminescence technology (SECTOR Imager 2400, Meso Scale Discovery, USA). In brief, this system uses multi-array plates fitted with multi-electrodes per well with each electrode being coated with a different catching antibody. The assay procedure then follows that of a classic sandwich ELISA with any of the analytes of interest captured on the relevant electrode. These captured analytes were then in turn detected by a secondary analyte-specific ruthenium-conjugated antibody, which is capable of emitting light after electrochemical stimulation. A particular advantage of this system is the ability to measure different circulating biomarkers of endothelial dysfunction and/or low-grade inflammation simultaneously in relatively small (25 or $50 \mu \mathrm{l})$ serum samples. In our laboratory, intra- and inter-assay CV were for sVCAM-1, 3.8 and $6.9 \%$; for sE-selectin, 4.1 and $8.6 \%$; for STM, 2.5 and 8.1\%; for sICAM-1, 2.6 and $6.0 \%$; for CRP, 2.3 and $4.3 \%$; for SAA, 4.5 and 9.0\%; for IL-6, 6.3 and $17.5 \%$; for IL-8, 6.9 and 7.3\%; and for TNF- $\alpha, 5.9$ and $12.6 \%$, respectively. We also measured IL-1 $\beta$ using the above system, but levels were below the detection limit in 107 participants (resulting in intra- and inter-assay CV of 20.0 and $32.2 \%$, respectively) and therefore we excluded IL-1 $\beta$ from the present investigation. In addition, a plasma marker of endothelial dysfunction ( $v W f$ ) was determined in citrated plasma by means of ELISA. Briefly, vWf rabbit antibody was used as a catching antibody and a peroxidase-conjugate rabbit antibody as detecting antibody. ${ }^{27}$ Levels were expressed as a percentage of $\mathrm{vWf}$ detected in pooled citrated plasma of healthy volunteers. Intra- and inter-assay CV for vWf were 3.8 and $7.4 \%$, respectively.

\section{Other measurements}

We measured weight, height and blood pressure, and serum total cholesterol, HDL and LDL cholesterol, and triglycerides, calculated body mass index (BMI) and obtained information on smoking behaviour and daily physical activity, as described in detail elsewhere. ${ }^{19,28,29}$ In addition, insulin was determined in serum samples by the multiarray detection system as described above. Intra- and inter-assay CV for insulin were 3.5 and $6.3 \%$, respectively.

\section{Statistical analyses}

First, we used linear regression analysis to examine the relationship between food consumption at the age of 36 years (2000) and changes in circulating biomarkers of endothelial dysfunction and low-grade inflammation between the ages of 36 years 
(2000) and 42 years (2006). We specifically investigated fish, fruit, vegetable, alcoholic beverage and dairy product consumption as these food groups have (repeatedly) been associated with circulating biomarkers of endothelial dysfunction and low-grade inflammation as part of a healthy diet. ${ }^{16-18}$ In a series of secondary analyses, we repeated the above analyses on a nutrient compound level to further investigate any associations between food groups and changes in concentration of circulating biomarkers of ED and LGI.

For reasons of statistical efficiency and to reduce the influences of the biological variability of each measure ${ }^{30}$, an overall Z-score was determined for both endothelial dysfunction and low-grade inflammation, according to predefined clusters of conceptually related circulating biomarkers. ${ }^{2,30}$ The overall Z-scores were calculated as follows: for each individual circulating biomarker, a Z-score was calculated according to the formula: (individual value - population mean)/population standard deviation (with the population mean and standard deviation for each individual circulating biomarker based on its average of the 2000 and 2006 follow-up examinations). The resulting individual circulating biomarker Z-scores were then averaged into the overall Z-score for both endothelial dysfunction and low-grade inflammation. The endothelial dysfunction overall Z-score consisted of the circulating biomarkers VWf, sVCAM-1, sE-selectin, STM, sICAM-1; and the low-grade inflammation overall Z-score consisted of the circulating biomarkers CRP, SAA, IL-6, IL-8, TNF- $\alpha$ and sICAM-1 (both monocytes and the endothelium express SICAM-1 and therefore sICAM-1 was included in both overall Z-scores). ${ }^{6}$ The changes in endothelial dysfunction or low-grade inflammation were calculated as the overall Z-score in $\mathbf{2 0 0 6}$ minus the overall Z-score in 2000.

First, we investigated the associations between each food group (fish, fruit, vegetables, alcoholic beverages and dairy products) and the change in overall Z-scores for endothelial dysfunction and low-grade inflammation, adjusted for sex and energy intake (models 1). Second, these associations were further adjusted for lifestyle risk factors [BMI, smoking behaviour (yes/no), alcohol consumption (none, low and high consumers) and physical activity (models 2)], and third, these associations were additionally adjusted for each of the other food groups (models 3 ).

To facilitate the interpretation of the changes in Z-scores, individuals were categorized as those who increased (change in Z-score $\geq+0.3 S D ; n=79$ for endothelial dysfunction and $n=64$ for low-grade inflammation), vs. those who remained stable or decreased (change in Z-score <+0.3SD; $n=222$ for endothelial dysfunction and $n=237$ for lowgrade inflammation) over the 6 -year follow-up. We then performed logistic regression analyses in order to directly compare individuals' likelihood of an increased Z-score (vs. stable or decreased) expressed in terms of an odds ratio (OR), across tertiles of fish consumption (independent variable).

We investigated whether EPA+DHA intake was associated with endothelial dysfunction and/or low-grade inflammation (nutrient residual method). ${ }^{31}$ In addition, we investigated to what extent the association between fish and endothelial dysfunction and/or low-grade inflammation could be explained by intake of EPA+DHA, 
because fish is the main source of EPA+DHA ${ }^{26}$ and the beneficial effects of fish intake have been attributed to EPA+DHA. ${ }^{32}$

All analyses were performed with the Statistical Package for Social Sciences (SPSS Inc, version 15, Chicago, IL, USA). Outcome variables with a skewed distribution were logtransformed in order to meet normality criteria (EPA+DHA intake, CRP, IL-6 and TNF- $\alpha$ ). We used Student's $t$ or $\chi^{2}$ tests, as appropriate, to compare between individuals with or without (serum and plasma) biomarker data at both moments. Data are presented as mean \pm SD or median (IQR) for skewed variables or as percentages and regression coefficients $(\beta)$ with their $95 \% \mathrm{Cl}$. A two-sided p-value $<0.05$ was considered statistically significant.

\section{Results}

The study population was young and relatively healthy, as $\mathrm{BMI}$, blood pressure and cholesterol levels were low (Table 4.1).

The 301 participants in the current study did not differ from those who only attended the 2000 follow-up ( $n=72$ ) except for smoking behaviour [35\% smoked (for $n=72$ ) vs. $21 \%$ (for $n=301$ ); $p=0.02$; other data not shown].

\section{Associations between food consumption and changes in endothelial dysfunction and low-grade inflammation}

Total fish consumption (per $100 \mathrm{~g} / \mathrm{wk}$ ) was inversely associated with a change in the endothelial dysfunction Z-score (higher scores associated with greater risk of incident CVD) [ $\beta(95 \% \mathrm{Cl}):-0.05(-0.09 ;-0.01), p=0.010$ (Table 4.2, model 1)]. Similar results were obtained between total fish consumption and the change in the low-grade inflammation Z-score [-0.04 (-0.08;0.004), p=0.079 (Table 4.2, model 1)]. The strength of these associations ( $\beta$ and $95 \% \mathrm{Cl}$ ) did not materially change after additional adjustment for lifestyle risk factors (Table 4.2, model 2) and other food groups (Table 4.2, model 3).

Compared with individuals in the highest tertile of fish consumption reference category: [median (IQR)] 200 (163-309) g\}, those in the second [75 (50-100) g] and first tertiles $[0.0(0.0-0.0) \mathrm{g}]$ had greater odds for increases in endothelial dysfunction [OR=1.92 (0.94;3.94), $p=0.076$ and $O R=2.66$ (1.31;5.37), $p=0.007$; $p$-trend=0.007], and similarly for increases in low-grade inflammation [OR=2.11 $(0.99 ; 4.48), p=0.053$ and OR=2.83 (1.35;5.96), $p=0.006 ; p$-trend=0.006] (Figure 4.1).

The above models ( 1 and 2 only) were then repeated with EPA+DHA intake (Table 4.2). The results showed an inverse association for EPA+DHA intake with changes in the endothelial dysfunction Z-score $[-0.11(-0.16 ;-0.05), p<0.001]$ and in the low-grade inflammation Z-score [-0.06 $(-0.12 ; 0.006), p=0.075]$. The strength of these associations $(\beta$ and $95 \% \mathrm{Cl}$ ) did not materially change after additional adjustment for lifestyle risk factors (Table 4.2, models 2 ) and other nutrients (Table 4.2, models 3). 
Table 4.1 Characteristics of the study population in 2000 and serum biomarkers in 2000 and $2006^{1,2}$.

\begin{tabular}{|c|c|c|}
\hline Characteristics & 2000 & 2006 \\
\hline Age, years & $36.5 \pm 0.63$ & \\
\hline Male, \% & 46.5 & \\
\hline $\mathrm{BMI}, \mathrm{kg} / \mathrm{m}^{2}$ & $24.1 \pm 3.1$ & \\
\hline Systolic blood pressure, $\mathrm{mmHg}$ & $131 \pm 13$ & \\
\hline Diastolic blood pressure, $\mathrm{mmHg}$ & $85.6 \pm 10.3$ & \\
\hline Serum HDL cholesterol, $\mathrm{mmol} / \mathrm{l}$ & $1.41 \pm 0.36$ & \\
\hline Serum LDL cholesterol, mmol// & $3.02 \pm 0.84$ & \\
\hline Serum triglycerides, $\mathrm{mmol} / \mathrm{l}$ & $1.0(0.8-1.5)$ & \\
\hline Serum insulin, $\mathrm{pmol} / \mathrm{l}$ & $90.4(55.1-172)$ & \\
\hline Smoking behaviour (yes/no), \% & 21.3 & \\
\hline Daily physical activity ${ }^{3}, \times 1000$ METs/wk & $4.14(2.61-6.36)$ & \\
\hline Alcohol (none/ low/ high), \% & $13.6 ; 52.5 ; 33.9$ & \\
\hline \multicolumn{3}{|l|}{ Food groups } \\
\hline Fish, g/wk & $75(0.0-163)$ & \\
\hline Fat fish, g/wk & $0(0-50.0)$ & \\
\hline Lean fish, $\mathrm{g} / \mathrm{wk}$ & $44(0-100)$ & \\
\hline Fruit, g/d & $143(64-232)$ & \\
\hline Vegetables, g/d & $119(82-172)$ & \\
\hline Alcoholic beverages, I/wk & $0.75(0.15-2.11)$ & \\
\hline Dairy products, $\mathrm{g} / \mathrm{d}$ & $510 \pm 334$ & \\
\hline \multicolumn{3}{|l|}{ Nutrient intake } \\
\hline Energy, MJ/d & $11.0 \pm 3.0$ & \\
\hline Carbohydrate, g/d & $297 \pm 87$ & \\
\hline Fat, g/d & $106 \pm 36$ & \\
\hline Protein, g/d & $99 \pm 26$ & \\
\hline Alcohol (ethanol), g/d & $8.6(1.6-20.8)$ & \\
\hline$E P A, g / d$ & $0.33(0.05-1.19)$ & \\
\hline $\mathrm{DHA}, \mathrm{g} / \mathrm{d}$ & $0.84(0.33-2.19)$ & \\
\hline$E P A+D H A, g / d$ & $1.17(0.37-3.51)$ & \\
\hline \multicolumn{3}{|l|}{ Circulating biomarkers } \\
\hline$v W f^{4}, \%$ & $103 \pm 40$ & $114 \pm 43$ \\
\hline sE-selectin, $\mu \mathrm{g} / \mathrm{I}$ & $10.4 \pm 4.6$ & $10.0 \pm 4.6$ \\
\hline $\mathrm{sTM}, \mu \mathrm{g} / \mathrm{l}$ & $2.46 \pm 0.65$ & $2.52 \pm 0.62$ \\
\hline sVCAM-1, $\mu \mathrm{g} / \mathrm{I}$ & $332 \pm 79$ & $332 \pm 68$ \\
\hline sICAM-1, $\mu \mathrm{g} / \mathrm{l}$ & $203 \pm 51$ & $201 \pm 41$ \\
\hline $\mathrm{CRP}, \mathrm{mg} / \mathrm{l}$ & $0.77(0.31-2.06)$ & $0.75(0.34-1.71)$ \\
\hline $\mathrm{SAA}, \mathrm{mg} / \mathrm{l}$ & $1.21(0.70-2.23)$ & $1.33(0.73-2.37)$ \\
\hline IL-6, ng/I & $2.3(1.7-3.7)$ & $2.4(1.8-3.8)$ \\
\hline $\mathrm{IL}-8, \mathrm{ng} / \mathrm{I}$ & $9.2 \pm 3.4$ & $9.6 \pm 4.1$ \\
\hline TNF- $\alpha, n g / l$ & $9.4 \pm 3.4$ & $9.1 \pm 3.4$ \\
\hline
\end{tabular}

${ }^{1}$ Values are means \pm standard deviation, median (interquartile range) or percentages as appropriate, $\mathrm{n}=301 .{ }^{2}$ EPA, eicosapentaenoic acid; DHA, docosapentaenoic acid; CRP, C-reactive protein; SAA, serum amyloid A; sE-selectin, soluble endothelial selectin; sICAM-1 soluble intercellular adhesion molecule 1; sTM, soluble thrombomodulin; sVCAM-1, soluble vascular cell adhesion molecule 1; vWf von Willebrand factor; IL-6, interleukin 6; IL-8, interleukin 8; TNF- $\alpha$, tumour necrosis factor alpha. ${ }^{3}$ METs; metabolic equivalents score (including average weekly time spent on different activities multiplied by their intensity). ${ }^{4}$ Measured in plasma. 
Table 4.2 Associations between fish consumption and changes in endothelial dysfunction and low-grade inflammation Z-scores during a 6-year follow-up in healthy adults, with higher scores associated with greater risk of incident CVD.

\begin{tabular}{|c|c|c|c|c|c|c|}
\hline \multirow[b]{2}{*}{ Models } & \multicolumn{3}{|c|}{ Change in ED Z-score } & \multicolumn{3}{|c|}{ Change in LGI Z-score } \\
\hline & $\beta^{1}$ & $95 \% \mathrm{Cl}$ & $\mathrm{p}$-value & $\beta^{1}$ & $95 \% \mathrm{Cl}$ & $p$-value \\
\hline 1. total fish ${ }^{3}$ & -0.05 & -0.09 to -0.01 & 0.010 & -0.04 & -0.08 to 0.004 & 0.079 \\
\hline 2. total fish ${ }^{4}$ & -0.06 & -0.09 to -0.02 & 0.005 & -0.04 & -0.08 to 0.001 & 0.057 \\
\hline 3. total fish ${ }^{5}$ & -0.06 & -0.10 to -0.02 & 0.003 & -0.05 & -0.09 to -0.003 & 0.036 \\
\hline 1. $E P A+D H A^{2,3}$ & -0.11 & -0.16 to -0.05 & $<0.001$ & -0.06 & -0.12 to 0.001 & 0.075 \\
\hline 2. $\mathrm{EPA}+\mathrm{DHA}^{2,4}$ & -0.12 & -0.18 to -0.06 & $<0.001$ & -0.07 & -0.13 to -0.004 & 0.039 \\
\hline 3. $E P A+D H A^{2,6}$ & -0.13 & -0.19 to -0.07 & $<0.001$ & -0.09 & -0.16 to -0.02 & 0.013 \\
\hline
\end{tabular}

${ }^{1}$ Values are regression coefficients $(\beta)$ that represent the change in endothelial dysfunction (ED) and lowgrade inflammation (LGI) Z-scores (in SD) over a 6-year period per $100 \mathrm{~g} /$ wk of fish consumption or per one unit increase in energy adjusted EPA+DHA intake; $(n=301) .{ }^{2}$ EPA+DHA represent the energy-adjusted combined intake of eicosapentaenoic acid and docosapentaenoic acid. ${ }^{3}$ model 1 adjusted for sex and energy intake. ${ }^{4}$ model 2 additionally adjusted for BMI, smoking behaviour, alcohol (categories), physical activity. ${ }^{5}$ model 3 for total fish additionally adjusted for fruit, vegetables, alcoholic beverages and dairy products. ${ }^{6}$ model 3 for EPA+DHA additionally adjusted for protein, carbohydrate, dietary cholesterol, alcohol (nutrient) and $\alpha$-linolenic acid.

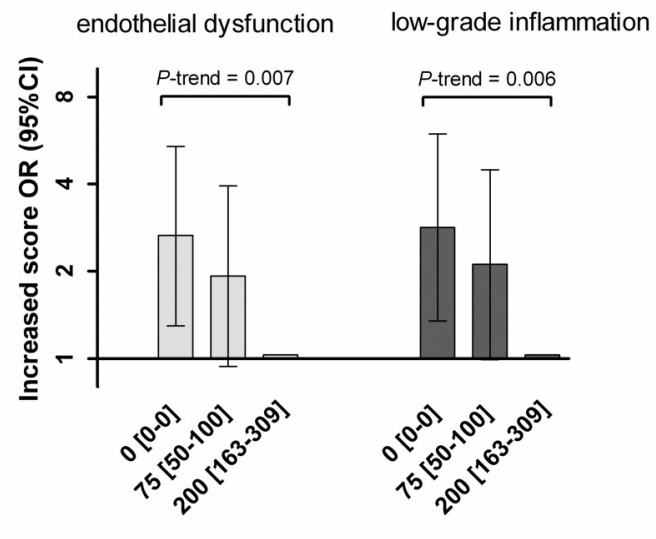

Fish intake, g/wk

Figure 4.1 Associations between fish consumption and increased circulating biomarkers of endothelial dysfunction and low-grade inflammation in healthy adults. Results are adjusted for sex, energy intake, body mass index, smoking behaviour, alcohol categories, physical activity, fruit, vegetables, alcoholic beverages, and dairy product consumption. 
The associations between fish consumption and the changes in endothelial dysfunction and low-grade inflammation overall Z-scores (Table 4.3, model 1) were attenuated by $83 \%$ and $40 \%$ when further adjusted for EPA and DHA, respectively (Table 4.3, model 2). This indicates that the associations between fish consumption and the changes in endothelial dysfunction and low-grade inflammation overall Z-score were explained, to a considerable extent, by the intake of EPA and DHA No statistically significant associations were observed between the consumption of fruit, vegetables, alcoholic beverages or dairy products and any changes in endothelial dysfunction or low-grade inflammation (please see Appendix, Table S4.1).

Table 4.3 Extent of mediation by EPA and DHA in the associations between fish consumption and changes in endothelial dysfunction and low-grade inflammation overall Z-scores in healthy adults.

\begin{tabular}{|c|c|c|c|c|c|c|c|c|}
\hline \multirow[b]{2}{*}{ Models } & \multicolumn{4}{|c|}{ Change in ED Z-score } & \multicolumn{4}{|c|}{ Change in LGI Z-score } \\
\hline & $\beta^{1}$ & $95 \% \mathrm{Cl}$ & $p$-value & $\begin{array}{c}\text { Mediation } \\
(\%)^{4} \\
\end{array}$ & $\beta^{1}$ & $95 \% \mathrm{Cl}$ & $\mathrm{p}$-value & $\begin{array}{c}\text { Mediation } \\
(\%)^{4} \\
\end{array}$ \\
\hline 1. total fish ${ }^{2}$ & -0.06 & -0.10 to -0.02 & 0.003 & & -0.05 & -0.09 to -0.003 & 0.036 & \\
\hline 2. $1+E P A+D H A^{3}$ & -0.01 & -0.06 to 0.05 & 0.764 & $83^{4}$ & -0.03 & -0.09 to 0.03 & 0.371 & $40^{4}$ \\
\hline
\end{tabular}

${ }^{1}$ Values are regression coefficients $(\beta)$ that represent the change in endothelial dysfunction (ED) and lowgrade inflammation (LGI) Z-scores (in SD) over a 6-year period per $100 \mathrm{~g} /$ wk of fish consumption; $(n=301)$. ${ }^{2}$ model adjusted for sex, energy intake, BMI, smoking behaviour, alcohol (categories), physical activity, fruit, vegetables, alcoholic beverages and dairy products. ${ }^{3}$ the model for total fish was additionally adjusted for EPA+DHA intake, which represents the energy-adjusted combined intake of eicosapentaenoic acid and docosapentaenoic acid. ${ }^{4}$ the extent of "mediation" expressed in the percentage of change in the regression coefficient after additional adjustment for EPA+DHA.

\section{Additional analyses}

The results were not materially altered when we additionally adjusted the models for age, serum insulin level or the intake of vitamin $C, E$ and $\beta$-carotene (data not shown). We repeated the analyses with either fat fish or lean fish consumption. The results showed that fat fish consumption (per $100 \mathrm{~g} / \mathrm{wk}$ ) was inversely associated with a change in the endothelial dysfunction Z-score $[-0.11(-0.19 ;-0.02) ; p=0.012]$ and a change in the low-grade inflammation Z-score [-0.08 $(-0.17 ; 0.02) ; p=0.12]$, after adjustment for sex, energy intake, lifestyle risk factors and other food groups. Results for lean fish consumption (per $100 \mathrm{~g} / \mathrm{wk}$ ) were similar \{[change in the endothelial dysfunction Z-score $(-0.05 \quad(-0.11 ; 0.005), p=0.07]$ and change in the low-grade inflammation Z-score $[-0.03(-0.10 ; 0.03), p=0.33]\}$. When we forced fat and lean fish consumption into one model the results showed that fat and lean fish did not differ from one another in the magnitude of their associations with either endothelial dysfunction $(p=0.33)$ or low-grade inflammation $(p=0.26)$. 
Analyses of the associations between total fish consumption and the individual elements of the endothelial dysfunction and low-grade inflammation Z-scores showed that total fish consumption was inversely associated with changes in all individual circulating biomarkers (higher scores associated with greater risk of incident CVD), with significant changes for $\mathrm{vWf}(\mathrm{p}<0.001)$ and sTM $(p=0.034)$ (Figure 4.2).

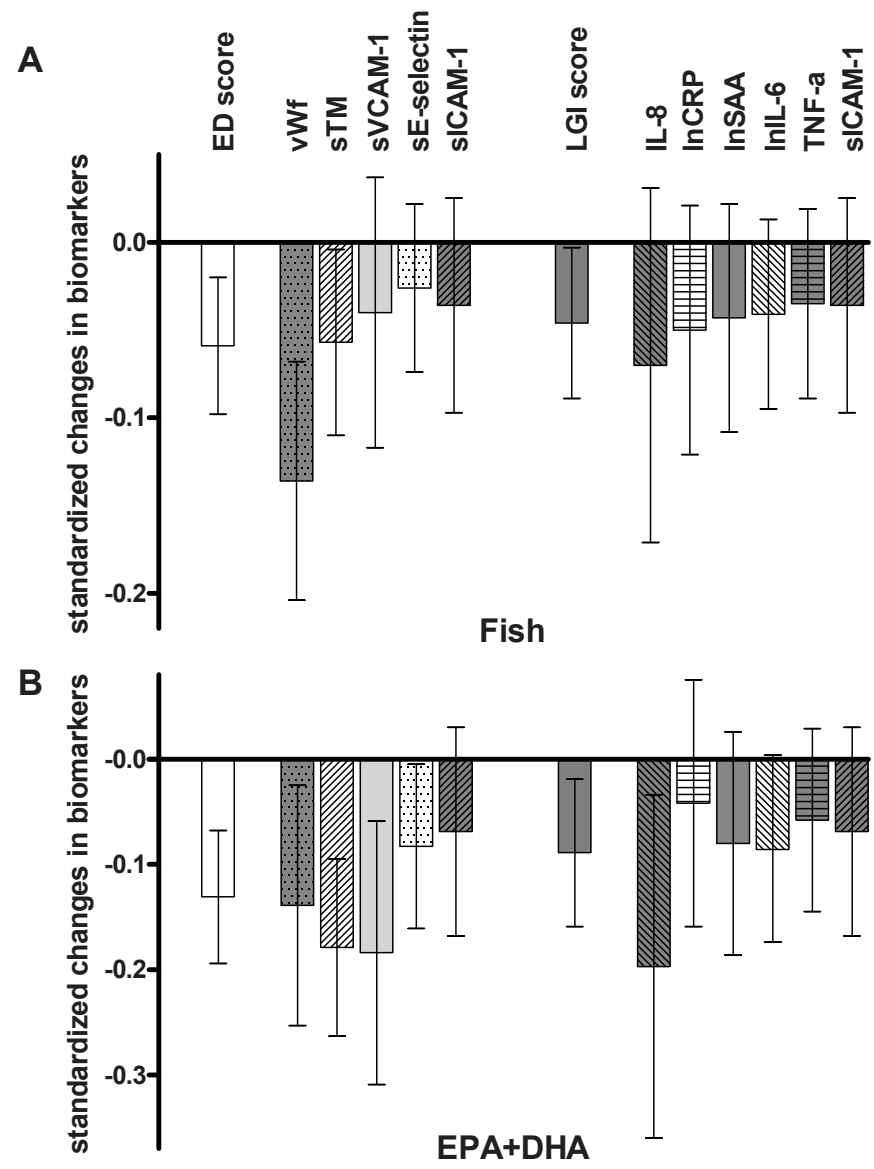

Figure 4.2 Fish consumption (A) and EPA+DHA intake (B) in healthy adults are associated with decreased circulating biomarkers of endothelial dysfunction (ED) and low-grade inflammation (LGI) over a 6-year follow-up. Results are adjusted for sex, energy intake, body mass index, smoking behaviour, alcohol categories, physical activity and, fruit, vegetables, alcoholic beverages and dairy product consumption for fish (A), or protein, carbohydrate, cholesterol, alcohol (nutrient) and $\alpha$-linolenic acid for EPA+DHA (B), respectively. CRP, C-reactive protein; SAA, serum amyloid $A$; sE-selectin, soluble endothelial selectin; sICAM-1 soluble intercellular adhesion molecule 1; sTM, soluble thrombomodulin; sVCAM-1, soluble vascular cell adhesion molecule 1; vWf von Willebrand factor; IL-6, interleukin 6; IL-8, interleukin 8; TNF- $\alpha$, tumour necrosis factor alpha. 
Analyses of the associations between EPA+DHA intake and the individual elements of the endothelial dysfunction and low-grade inflammation Z-scores showed that EPA+DHA intake was inversely associated with changes in all individual circulating biomarkers (higher levels associated with more CVD), with significant changes for $\mathrm{vWf}$ $(p=0.017)$, sTM $(p<0.001)$, sVCAM-1 $(p=0.004)$, sE-selectin $(p=0.038)$ and IL-8 $(p=0.018)$ (Figure 4.2).

When we replaced the changes in individual circulating biomarker Z-scores by absolute changes in individual circulating biomarkers and repeated the analyses, the results were similar (data not shown).

We used interaction terms to explore whether associations differed by sex or smoking behaviour. We found significant interaction terms for sex between fish consumption (as well as for EPA+DHA) and the change in the overall endothelial dysfunction Z-score $\left(p_{\text {interaction }}=0.02\right.$ and $p_{\text {interaction }}=0.01$, respectively) and for smoking between fish consumption and the change in the overall low-grade inflammation Z-score $\left(p_{\text {interaction }}=0.02\right)$. Stratified results showed a stronger association between fish consumption (as well as for EPA+DHA) and changes in the overall endothelial dysfunction Z-score in women as compared to men (please see Appendix, Table S4.2) and a stronger association between fish consumption and changes in the overall lowgrade inflammation Z-score in non-smokers as compared to smokers (please see Appendix, Table S4.3)

\section{Discussion}

The present investigation is the first to our knowledge to evaluate, in apparently healthy adults, the relationship between the consumption of fish, fruit, vegetables, alcoholic beverages and dairy products and the changes in an extensive array of serum or plasma biomarkers of both endothelial dysfunction and low-grade inflammation. The study had three main findings. First, fish consumption was associated with decreased endothelial dysfunction and decreased low-grade inflammation, independent of lifestyle risk factors and other food groups. Second, the combined nutrient compounds EPA and DHA were associated with decreased endothelial dysfunction and decreased low-grade inflammation, independent of lifestyle risk factors and other nutrients. Finally, the combined nutrient compounds EPA and DHA explained $83 \%$ of decreased endothelial dysfunction and $40 \%$ of decreased low-grade inflammation associated with greater fish consumption.

The present prospective investigation expands previous knowledge on the relationship between food intake and circulating biomarkers of endothelial dysfunction and low-grade inflammation, as previous studies were cross-sectional by design, investigated less circulating biomarkers, and/or dealt with middle-aged to elderly individuals only. ${ }^{16-18}$ Nevertheless, on aggregate, previous studies ${ }^{16-18}$ showed that diet scores including high consumption of fish, fruit and vegetables, and with 
moderate alcohol intake were associated with lower serum levels of (individual) markers of endothelial dysfunction, such as sE-selectin ${ }^{16,17}$, sICAM-1 ${ }^{16,18}$ and sVCAM- $1^{16}$ and lower serum levels of (individual) markers of low-grade inflammation, such as $\mathrm{CRP}^{16-18}$, IL-6 ${ }^{16,18}$ and SICAM-1 ${ }^{16,18}$, whereas high-fat dairy product consumption was associated with higher serum levels of sICAM-1. ${ }^{18}$ Importantly, observational studies have shown that higher concentrations of these circulating biomarkers of endothelial dysfunction and low-grade inflammation are associated with greater risk of incident CVD..$^{1-5}$ In addition to the above, the current results provide evidence that diet, and in particular fish consumption, affects serum and/or plasma biomarkers of endothelial dysfunction and low-grade inflammation. We show that individuals who consume no fish have greater odds to increased (worse) endothelial dysfunction and low-grade inflammation scores during a 6-year follow-up period, as compared with individuals who consume fish (Figure 4.1). Taken together, these studies ${ }^{16-18}$ and the present support the hypothesis that a diet rich in fish may influence the development of CVD by modulating endothelial dysfunction and lowgrade inflammation.

When we analyzed fat and lean fish separately, fat fish consumption seemed more strongly associated with decreased endothelial dysfunction and decreased low-grade inflammation as compared to lean fish consumption. When we further tested (between fat and lean fish) whether the strength of these associations differed from one another, we found that these did not statistically differ $(p=0.33$ and $p=0.26$, respectively). The present investigation, therefore, does not support the concept that fat, as compared to lean, fish consumption is more important with regard to a decrease of endothelial dysfunction and low-grade inflammation, despite the fact that fat fish contains a greater EPA and DHA content. ${ }^{26}$

EPA and DHA may modify endothelial dysfunction and low-grade inflammation through altered cell membrane stability, endothelial nitric oxide synthase activity, and prostaglandin synthesis. $^{33,34}$ Additionally, EPA and DHA may modify low-grade inflammation through the inhibition of intracellular inflammatory signalling pathways and the reduction of oxidative stress. ${ }^{34}$ The present results showed indeed that, on a nutrient compound level, EPA and DHA were associated with decreased endothelial dysfunction and low-grade inflammation. EPA and DHA explained $83 \%$ of the associations between fish consumption and the change in endothelial dysfunction and $40 \%$ of the association between fish consumption and the change in low-grade inflammation.

The association between fish consumption and change in endothelial dysfunction was stronger in women as compared to men and the association between fish consumption and change in low-grade inflammation was stronger in non-smokers as compared to smokers. The present investigation neither can explain these observations nor can exclude the play of chance. Therefore, these exploratory results should be interpreted with caution and serve merely as hypothesis generating for future studies. 
As diet is an important determinant of insulin resistance and insulin resistance itself is associated with elevated concentrations of serum or plasma markers of endothelial dysfunction and low-grade inflammation ${ }^{2,3,30}$, we additionally adjusted the results for serum insulin. This did not materially change our results. The above suggests that fish consumption is associated with changes in endothelial dysfunction and low-grade inflammation independent of insulin resistance.

We determined an overall Z-score for both endothelial dysfunction and low-grade inflammation, according to predefined clusters of conceptually related circulating biomarkers. The use of overall Z-scores is statistically efficient as this approach reduces the number of associations that need to be (statistically) explored and thus reduces the chance of spurious findings. In addition, the use of Z-scores reduces the influences of biological variability of each measure and, the sum of conceptually related circulating biomarkers yields more information than each individual circulating biomarker alone as shown in Figure 4.2.

Although we hypothesized that a high consumption of fruit and/or vegetables, a moderate consumption of alcohol and/or a low consumption of dairy products were associated with a decrease of endothelial dysfunction and low-grade inflammation ${ }^{10-}$ ${ }^{14}$, we did not observe any associations between the consumptions of these food groups and changes in endothelial dysfunction or low-grade inflammation. When we re-analyzed the data cross-sectionally in order to facilitate comparison with previous cross-sectional studies ${ }^{10-14,35-38}$ we found that the participants in this cohort that consumed more fruit, vegetables and alcoholic beverages had lower circulating levels of biomarkers of endothelial dysfunction and low-grade inflammation [statistically significant for the association between vegetable consumption and endothelial dysfunction (data not shown)]. In the current data set we cannot exclude that the variation between persons in the consumption of fruit, vegetables, alcoholic beverages and dairy products was too small to reveal any associations or alternatively that effects of these food groups may only be seen later in life. In addition, the nature of the study population, which consisted of healthy adults, could have led to an underestimation of the associations, as effects of food groups on changes in endothelial dysfunction and low-grade inflammation are, most likely, small at this age. The present investigation had some limitations. First, our findings were confined to participants attending the 2000 and 2006 follow-up examinations in whom complete data on circulating biomarkers could be assessed. Selection could have biased our findings if persons refused further participation (at any time-point during the 30-year study) based on the association between fish consumption (at the age of 36 years) and changes in endothelial dysfunction and low-grade inflammation (between the ages of 36 years and 42 years), a scenario that we consider highly unlikely. Second, intrinsic to an overall Z-score is the assumption that each circulating biomarker in the Z-score carries similar weight. Consequently, this approach may not optimally reflect pathophysiology as it is unknown if the relationship between food intake and circulating biomarkers as a proxy for cardiovascular disease is similar for each 
circulating biomarker. Third, we cannot fully exclude that other forms of behaviour that favour health might have affected our findings, although the present results remained unchanged after additional adjustment for BMI, smoking behaviour, physical activity, alcohol consumption, other food groups or nutrients, serum insulin concentrations and dietary vitamins. Fourth, extensive dietary data on food group level were available in 2000 only. Recent data from the EPIC-Heidelberg cohort have shown that dietary data assessed only once can be used as long-term exposure variable to investigate diet and disease associations as dietary habits remain stable over the $3^{\text {rd }}$ and $4^{\text {th }}$ decades of life. ${ }^{39}$ It is therefore unlikely that the present results have been influenced by the assessment of diet in 2000 only. Nevertheless, two dietary measurements to assess an individual's diet would have provided a more accurate dietary classification. Consequently, we cannot fully exclude that measuring food intake in $\mathbf{2 0 0 0}$ only might have led us to underestimate the relationship between food intake and endothelial dysfunction, and low-grade inflammation. ${ }^{39}$ Fifth, no data on the female hormonal cycle were available and therefore we were unable to investigate whether this cycle played a role in the associations between food consumption and changes in endothelial dysfunction or low-grade inflammation. Finally, the present investigation was limited to the consumption of food groups and primarily macronutrients and did not concentrate upon e.g. polyphenols and flavonoids, which have recently attracted attention as these micronutrients have been shown to affect endothelial dysfunction. ${ }^{40-44}$

In conclusion, in young, apparently healthy adults, the consumption of fish but not fruit, vegetables, alcoholic beverages or dairy products, is associated with decreased endothelial dysfunction and low-grade inflammation during a 6-year follow-up. This suggests that fish consumption may affect the development of CVD through modification of endothelial dysfunction and low-grade inflammation. 


\section{References}

1. Blankenberg S, Rupprecht HJ, Bickel C, Peetz D, Hafner G, Tiret L, Meyer J. Circulating cell adhesion molecules and death in patients with coronary artery disease. Circulation. 2001;104:1336-1342.

2. de Jager J, Dekker JM, Kooy A, Kostense PJ, Nijpels G, Heine RJ, Bouter LM, Stehouwer CD. Endothelial dysfunction and low-grade inflammation explain much of the excess cardiovascular mortality in individuals with type 2 diabetes: the Hoorn Study. Arterioscler Thromb Vasc Biol. 2006;26:1086-1093.

3. Jager A, van Hinsbergh VW, Kostense PJ, Emeis JJ, Yudkin JS, Nijpels G, Dekker JM, Heine RJ, Bouter LM, Stehouwer CD. von Willebrand factor, C-reactive protein, and 5-year mortality in diabetic and nondiabetic subjects: the Hoorn Study. Arterioscler Thromb Vasc Biol. 1999;19:3071-3078.

4. Morange PE, Simon C, Alessi MC, Luc G, Arveiler D, Ferrieres J, Amouyel P, Evans A, Ducimetiere P, Juhan-Vague I. Endothelial cell markers and the risk of coronary heart disease: the Prospective Epidemiological Study of Myocardial Infarction (PRIME) study. Circulation. 2004;109:1343-1348.

5. Ridker PM, Hennekens $\mathrm{CH}$, Buring JE, Rifai N. C-reactive protein and other markers of inflammation in the prediction of cardiovascular disease in women. N Engl J Med. 2000;342:836-843.

6. Schram MT, Stehouwer CD. Endothelial dysfunction, cellular adhesion molecules and the metabolic syndrome. Horm Metab Res. 2005;37 Suppl 1:49-55.

7. Ross R. Atherosclerosis--an inflammatory disease. N Engl J Med. 1999;340:115-126.

8. Vischer UM. von Willebrand factor, endothelial dysfunction, and cardiovascular disease. J Thromb Haemost. 2006;4:1186-1193.

9. Lichtenstein $\mathrm{AH}$, Appel $\mathrm{L}$, Brands $\mathrm{M}$, Carnethon $\mathrm{M}$, Daniels S, Franch HA, Franklin B, Kris-Etherton $\mathrm{P}$, Harris WS, Howard B, Karanja N, Lefevre M, Rudel L, Sacks F, Van Horn L, Winston M, Wylie-Rosett J. Diet and lifestyle recommendations revision 2006: a scientific statement from the American Heart Association Nutrition Committee. Circulation. 2006;114:82-96.

10. Harriss LR, English DR, Powles J, Giles GG, Tonkin AM, Hodge AM, Brazionis L, O'Dea K. Dietary patterns and cardiovascular mortality in the Melbourne Collaborative Cohort Study. Am J Clin Nutr. 2007;86:221-229.

11. Hu FB, Rimm EB, Stampfer MJ, Ascherio A, Spiegelman D, Willett WC. Prospective study of major dietary patterns and risk of coronary heart disease in men. Am J Clin Nutr. 2000;72:912-921.

12. Knoops KT, de Groot LC, Kromhout D, Perrin AE, Moreiras-Varela O, Menotti A, van Staveren WA. Mediterranean diet, lifestyle factors, and 10-year mortality in elderly European men and women: the HALE project. JAMA. 2004;292:1433-1439.

13. Mitrou PN, Kipnis V, Thiebaut AC, Reedy J, Subar AF, Wirfalt E, Flood A, Mouw T, Hollenbeck AR, Leitzmann MF,Schatzkin A. Mediterranean dietary pattern and prediction of all-cause mortality in a US population: results from the NIH-AARP Diet and Health Study. Arch Intern Med. 2007;167: 2461-2468.

14. Panagiotakos D, Pitsavos C, Chrysohoou C, Palliou K, Lentzas I, Skoumas I, Stefanadis C. Dietary patterns and 5-year incidence of cardiovascular disease: a multivariate analysis of the ATTICA study. Nutr Metab Cardiovasc Dis. 2009;19:253-63.

15. Esposito K, Marfella R, Ciotola M, Di Palo C, Giugliano F, Giugliano G, D'Armiento M, D'Andrea F, Giugliano D. Effect of a mediterranean-style diet on endothelial dysfunction and markers of vascular inflammation in the metabolic syndrome: a randomized trial. JAMA. 2004;292:1440-6.

16. Fung TT, McCullough ML, Newby PK, Manson JE, Meigs JB, Rifai N, Willett WC, Hu FB. Diet-quality scores and plasma concentrations of markers of inflammation and endothelial dysfunction. Am J Clin Nutr. 2005;82:163-173.

17. Lopez-Garcia E, Schulze MB, Fung TT, Meigs JB, Rifai N, Manson JE, Hu FB. Major dietary patterns are related to plasma concentrations of markers of inflammation and endothelial dysfunction. Am J Clin Nutr. 2004;80:1029-1035.

18. Nettleton JA, Steffen LM, Mayer-Davis EJ, Jenny NS, Jiang R, Herrington DM, Jacobs DR, Jr. Dietary patterns are associated with biochemical markers of inflammation and endothelial activation in the Multi-Ethnic Study of Atherosclerosis (MESA). Am J Clin Nutr. 2006;83:1369-1379. 
19. Ferreira I, Twisk JW, van Mechelen W, Kemper HC, Stehouwer CD. Development of fatness, fitness, and lifestyle from adolescence to the age of 36 years: determinants of the metabolic syndrome in young adults: the amsterdam growth and health longitudinal study. Arch Intern Med. 2005;165: 42-48.

20. de Vente W, Post GB, Twisk JW, Kemper HC, van Mechelen W. Effects of health measurements and health information in youth and young adulthood in dietary intake--20-y study results from the Amsterdam Growth and Health Longitudinal Study. Eur J Clin Nutr. 2001;55:819-823.

21. Kemper HC. Amsterdam Growth and Health Longitudinal Study (AGAHLS): A 23-Year Follow-up From Teenage to Adult About Lifestyle and Health. Basel, Switzerland: Karger; 2004.

22. Bakker I, Twisk JW, van Mechelen W, Mensink GB, Kemper HC. Computerization of a dietary history interview in a running cohort; evaluation within the Amsterdam Growth and Health Longitudinal Study. Eur J Clin Nutr. 2003;57:394-404.

23. Kromhout D, de Lezenne Coulander C, Obermann-de Boer GL, van Kampen-Donker M, Goddijn E, Bloemberg BP. Changes in food and nutrient intake in middle-aged men from 1960 to 1985 (the Zutphen Study). Am J Clin Nutr. 1990;51:123-129.

24. Burke BS. The dietary history as a tool in research. J Am Diet Assoc. 1947;1041-1046.

25. Mensink GB, Haftenberger M, Thamm M. Validity of DISHES 98, a computerised dietary history interview: energy and macronutrient intake. Eur J Clin Nutr. 2001;55:409-417.

26. NEVO. Nederlands voedingsstoffenbestand, NEVO-tabel 2006. The Hague: NEVO Foundation; 2006.

27. de Valk-de Roo GW, Stehouwer CD, Lambert J, Schalkwijk CG, van der Mooren MJ, Kluft C, Netelenbos C. Plasma homocysteine is weakly correlated with plasma endothelin and von Willebrand factor but not with endothelium-dependent vasodilatation in healthy postmenopausal women. Clin Chem. 1999;45:1200-1205.

28. Ferreira I, Twisk JW, Van Mechelen W, Kemper HC, Stehouwer CD. Current and adolescent levels of cardiopulmonary fitness are related to large artery properties at age 36: the Amsterdam Growth and Health Longitudinal Study. Eur J Clin Invest. 2002;32:723-731.

29. van Mechelen W, Twisk JW, Post GB, Snel J, Kemper HC. Physical activity of young people: the Amsterdam Longitudinal Growth and Health Study. Med Sci Sports Exerc. 2000;32:1610-1616.

30. Yudkin JS, Stehouwer CD, Emeis JJ, Coppack SW. C-reactive protein in healthy subjects: associations with obesity, insulin resistance, and endothelial dysfunction: a potential role for cytokines originating from adipose tissue? Arterioscler Thromb Vasc Biol. 1999;19:972-978.

31. Willett WC. Nutritional Epidemiology, 2th ed. New York, NY: Oxford University Press; 1998.

32. Geleijnse JM, Giltay EJ, Schouten EG, de Goede J, Oude Griep LM, Teitsma-Jansen AM, Katan MB, Kromhout D. Effect of low doses of n-3 fatty acids on cardiovascular diseases in 4,837 post-myocardial infarction patients: design and baseline characteristics of the Alpha Omega Trial. Am Heart J. 2010;159:539-46 e2.

33. Tull SP, Yates CM, Maskrey BH, O'Donnell VB, Madden J, Grimble RF, Calder PC, Nash GB, Rainger GE. Omega-3 Fatty acids and inflammation: novel interactions reveal a new step in neutrophil recruitment. PLoS Biol. 2009;7:e1000177.

34. Hall WL. Dietary saturated and unsaturated fats as determinants of blood pressure and vascular function. Nutr Res Rev. 2009;22:18-38.

35. Wannamethee SG, Lowe GD, Rumley A, Bruckdorfer KR, Whincup PH. Associations of vitamin C status, fruit and vegetable intakes, and markers of inflammation and hemostasis. Am J Clin Nutr. 2006;83:567-74; quiz 726-727.

36. Gao X, Bermudez OI, Tucker KL. Plasma C-reactive protein and homocysteine concentrations are related to frequent fruit and vegetable intake in Hispanic and non-Hispanic white elders. J Nutr. 2004;134:913-918.

37. Holt EM, Steffen LM, Moran A, Basu S, Steinberger J, Ross JA, Hong CP, Sinaiko AR. Fruit and vegetable consumption and its relation to markers of inflammation and oxidative stress in adolescents. J Am Diet Assoc. 2009;109:414-421.

38. Shai I, Rimm EB, Schulze MB, Rifai N, Stampfer MJ, Hu FB. Moderate alcohol intake and markers of inflammation and endothelial dysfunction among diabetic men. Diabetologia. 2004;47:1760-1767.

39. Nagel G, Zoller D, Ruf T, Rohrmann S, Linseisen J. Long-term reproducibility of a food-frequency questionnaire and dietary changes in the European Prospective Investigation into Cancer and Nutrition (EPIC)-Heidelberg cohort. Br J Nutr. 2007;98:194-200. 
40. Balzer J, Rassaf T, Heiss C, Kleinbongard P, Lauer T, Merx M, Heussen N, Gross HB, Keen CL, Schroeter $\mathrm{H}$, Kelm M. Sustained benefits in vascular function through flavanol-containing cocoa in medicated diabetic patients a double-masked, randomized, controlled trial. J Am Coll Cardiol. 2008;51: 2141-2149.

41. Buijsse B, Feskens EJ, Kok FJ, Kromhout D. Cocoa intake, blood pressure, and cardiovascular mortality: the Zutphen Elderly Study. Arch Intern Med. 2006;166:411-417.

42. Corti R, Flammer AJ, Hollenberg NK, Luscher TF. Cocoa and cardiovascular health. Circulation. 2009;119:1433-1441.

43. Heiss C, Dejam A, Kleinbongard P, Schewe T, Sies H, Kelm M. Vascular effects of cocoa rich in flavan-3-ols. JAMA. 2003;290:1030-1031.

44. Heiss C, Jahn S, Taylor M, Real WM, Angeli FS, Wong ML, Amabile N, Prasad M, Rassaf T, Ottaviani JI,Mihardja S, Keen CL, Springer ML, Boyle A, Grossman W, Glantz SA, Schroeter H, Yeghiazarians Y. Improvement of endothelial function with dietary flavanols is associated with mobilization of circulating angiogenic cells in patients with coronary artery disease. J Am Coll Cardiol. 2010;56: 218-224. 


\section{Appendix 4}

The Dutch food composition database contains 23 food groups, each with codes for specific kinds of foods, and covers the complete dietary intake in the Netherlands. For the present investigation, we used the following groups: fish, fruit, alcoholic beverages, and milk products and cheese for dairy products, and vegetables and legumes, but not potatoes, for vegetables. The computer-assisted crosscheck dietary history method (DISHES) was comprehensive and included 45 codes for specific kinds of fish, 48 for fruits, 18 for alcoholic beverages, 53 for milk products and 37 for cheese, and 81 for vegetables and 7 for legumes. With the amounts of these specific kinds of foods measured for each individual, the total consumptions of fish, fruit, vegetable, alcoholic beverage and dairy products were calculated. In addition, fish consumption was divided into fat fish (19 codes for specific kinds of fat fish) and lean fish (20 codes for specific kinds of lean fish), and included shellfish (6 codes). Furthermore, the nutrient compounds were calculated on the complete dietary data including all food groups present in the Dutch food composition database.

Table S4.1 Associations between food consumption and changes in endothelial dysfunction and lowgrade inflammation Z-scores during a 6-year follow-up, with higher scores associated with greater risk of incident CVD.

\begin{tabular}{|c|c|c|c|c|c|c|}
\hline \multirow[b]{2}{*}{ Models } & \multicolumn{3}{|c|}{ Change in ED Z-score } & \multicolumn{3}{|c|}{ Change in LGI Z-score } \\
\hline & $\beta^{1}$ & $95 \% \mathrm{Cl}$ & p-value & $\beta^{1}$ & $95 \% \mathrm{Cl}$ & p-value \\
\hline 1. total fruit ${ }^{2}$ & 0.004 & -0.003 to 0.01 & 0.234 & 0.005 & -0.002 to 0.01 & 0.143 \\
\hline 2. total fruit ${ }^{3}$ & 0.004 & -0.002 to 0.01 & 0.213 & 0.006 & -0.001 to 0.01 & 0.099 \\
\hline 3. total fruit ${ }^{4}$ & 0.005 & -0.002 to 0.01 & 0.134 & 0.006 & -0.001 to 0.01 & 0.092 \\
\hline 1. total vegetable ${ }^{2}$ & 0.001 & -0.008 to 0.01 & 0.769 & 0.002 & -0.009 to 0.01 & 0.740 \\
\hline 2. total vegetable ${ }^{3}$ & 0.001 & -0.008 to 0.01 & 0.794 & 0.003 & -0.007 to 0.01 & 0.525 \\
\hline 3. total vegetable $e^{5}$ & 0.000 & -0.01 to 0.01 & 0.990 & 0.002 & -0.009 to 0.01 & 0.778 \\
\hline 1. total alcoholic beverage ${ }^{2}$ & 0.001 & -0.002 to 0.005 & 0.475 & 0.000 & -0.004 to 0.004 & 0.969 \\
\hline 2. total alcoholic beverage ${ }^{3}$ & 0.001 & -0.004 to 0.005 & 0.797 & -0.001 & -0.006 to 0.004 & 0.654 \\
\hline 3. total alcoholic beverage ${ }^{6}$ & 0.001 & -0.003 to 0.006 & 0.542 & 0.000 & -0.005 to 0.005 & 0.891 \\
\hline 1. total dairy products ${ }^{2}$ & 0.002 & -0.001 to 0.005 & 0.170 & 0.001 & -0.002 to 0.004 & 0.431 \\
\hline 2. total dairy products ${ }^{3}$ & 0.002 & -0.001 to 0.005 & 0.176 & 0.001 & -0.002 to 0.004 & 0.402 \\
\hline 3. total dairy products ${ }^{7}$ & 0.002 & -0.001 to 0.005 & 0.160 & 0.001 & -0.002 to 0.004 & 0.464 \\
\hline
\end{tabular}

\footnotetext{
${ }^{1}$ Values are regression coefficients $(\beta)$ that represent the change in endothelial dysfunction (ED) and lowgrade inflammation (LGI) Z-scores (in SD) over a 6-year period per $100 \mathrm{~g} / \mathrm{wk}$ (or per $100 \mathrm{ml} /$ wk for alcoholic beverage) consumption of the food group; $(n=301) .{ }^{2}$ model 1 adjusted for sex and energy intake. ${ }^{3}$ model 2 additionally adjusted for body mass index, smoking behaviour, alcohol (categories), physical activity. ${ }^{4}$ model 3 for total fruit additionally adjusted for fish, vegetables, alcoholic beverages and dairy products. ${ }^{5}$ model 3 for total vegetable additionally adjusted for fish, fruit, alcoholic beverages and dairy products. ${ }^{6}$ model 3 for total alcoholic beverage additionally adjusted for fish, fruit, vegetables and dairy products. ${ }^{7}$ model 3 for total dairy products additionally adjusted for fish, fruit, vegetables and alcoholic beverages.
} 
Table S4.2 Sex-stratified associations between fish consumption and changes in the endothelial dysfunction Z-score during a 6-year follow-up, with higher scores associated with greater risk of incident CVD.

Change in ED Z-score

\begin{tabular}{|c|c|c|c|c|c|c|}
\hline \multirow[b]{3}{*}{ Models } & \multicolumn{6}{|c|}{ Change in ED Z-score } \\
\hline & \multicolumn{3}{|c|}{ Women } & \multicolumn{3}{|c|}{ Men } \\
\hline & $\beta^{1}$ & $95 \% \mathrm{Cl}$ & p-value & $\beta^{1}$ & $95 \% \mathrm{Cl}$ & p-value \\
\hline 1. total fish ${ }^{3}$ & -0.11 & -0.16 to -0.05 & $<0.001$ & -0.01 & -0.06 to 0.04 & 0.634 \\
\hline 2. total fish ${ }^{4}$ & -0.11 & -0.17 to -0.06 & $<0.001$ & -0.01 & -0.07 to 0.04 & 0.609 \\
\hline 3. total fish ${ }^{5}$ & -0.12 & -0.18 to -0.06 & $<0.001$ & -0.02 & -0.07 to 0.04 & 0.517 \\
\hline 1. $E P A+D H A^{2,3}$ & -0.19 & -0.26 to -0.11 & $<0.001$ & -0.02 & -0.11 to 0.07 & 0.629 \\
\hline 2. $E P A+D H A^{2,4}$ & -0.21 & -0.29 to -0.13 & $<0.001$ & -0.04 & -0.13 to 0.05 & 0.401 \\
\hline 3. $E P A+D H A^{2,6}$ & -0.22 & -0.30 to -0.14 & $<0.001$ & -0.05 & -0.15 to 0.05 & 0.352 \\
\hline
\end{tabular}

${ }^{1}$ Values are regression coefficients $(\beta)$ that represent the change in endothelial dysfunction (ED) Z-score (in $\mathrm{SD}$ ) over a 6-year period per $100 \mathrm{~g} / \mathrm{wk}$ of fish consumption or per one unit increase in energy-adjusted EPA+DHA intake; $¥ P<0.001,\left(n=161\right.$ women, $n=140$ men). ${ }^{2}$ EPA+DHA represent the energy-adjusted combined intake of EPA and DHA. ${ }^{3}$ models 1 adjusted for energy intake. ${ }^{4}$ models 2 additionally adjusted for body mass index, smoking behaviour, alcohol (categories), physical activity. ${ }^{5}$ models 3 for total fish additionally adjusted for fruit, vegetables, alcoholic beverages and dairy products. 6 models 3 for EPA+DHA additionally adjusted for protein, carbohydrate, dietary cholesterol, alcohol (nutrient) and $\alpha$-linolenic acid.

Table S4.3 Smoking-stratified associations between fish consumption and changes in the low-grade inflammation Z-score during a 6-year follow-up, with higher scores associated with greater risk of incident CVD.

\begin{tabular}{|c|c|c|c|c|c|c|}
\hline \multirow[b]{3}{*}{ Models } & \multicolumn{6}{|c|}{ Change in LGI Z-score } \\
\hline & \multicolumn{3}{|c|}{ non-smokers } & \multicolumn{3}{|c|}{ smokers } \\
\hline & $\beta^{1}$ & $95 \% \mathrm{Cl}$ & $p$-value & $\beta^{1}$ & $95 \% \mathrm{Cl}$ & $p$-value \\
\hline 1. total fish ${ }^{2}$ & -0.06 & -0.106 to -0.010 & 0.005 & 0.05 & -0.035 to 0.141 & 0.760 \\
\hline 2. total fish ${ }^{3}$ & -0.06 & -0.111 to -0.013 & 0.002 & 0.07 & -0.017 to 0.156 & 0.969 \\
\hline 3. total fish ${ }^{4}$ & -0.07 & -0.118 to -0.019 & 0.001 & 0.05 & -0.042 to 0.141 & 0.914 \\
\hline
\end{tabular}

${ }^{1}$ Values are regression coefficients $(\beta)$ that represent the change in low-grade inflammation (LGI) Z-score (in $\mathrm{SD}$ ) over a 6-year period per $100 \mathrm{~g} /$ wk of fish consumption; $+p \leq 0.01, * p \leq 0.05$; ( $=237$ non-smokers, $n=64$ smokers). ${ }^{2}$ models 1 adjusted for sex and energy intake. ${ }^{3}$ models 2 additionally adjusted for body mass index, smoking behaviour, alcohol (categories), physical activity. ${ }^{4}$ models 3 for total fish additionally adjusted for fruit, vegetables, alcoholic beverages and dairy products. 


\section{Chapter}

Alcohol and red wine consumption are associated with less endothelial dysfunction and less low-grade inflammation: The Hoorn Study

BCT van Bussel, RMA Henry, CG Schalkwijk, I Ferreira, JM Dekker, G Nijpels, EJM Feskens, CDA Stehouwer Manuscript in preparation 


\section{Abstract}

\section{Background}

Endothelial dysfunction and low-grade inflammation are key phenomena in the pathobiology of cardiovascular disease (CVD). Their dietary modification might explain the observed reduction in CVD that has been associated with a healthy diet rich in fruit, vegetables and fish, low in dairy products and with moderate alcohol and red wine consumption.

\section{Objective}

To investigate the associations between the above food groups and endothelial dysfunction and low-grade inflammation in a population-based cohort of older individuals.

\section{Methods}

Diet was measured by Food Frequency Questionnaire ( $n=801$; women=399; age 68.5 \pm 7.2 years). Endothelial dysfunction was determined (1) by combining von Willebrand factor, soluble vascular cell adhesion molecule 1 , soluble endothelial selectin, soluble thrombomodulin and soluble intercellular adhesion molecule 1 (sICAM-1), using Z-scores, into a circulating biomarker score and (2) by flow-mediated vasodilation (FMD); and low-grade inflammation by combining C-reactive protein, serum amyloid A, interleukin 6, interleukin 8, tumour necrosis factor $\alpha$ and sICAM-1 into a circulating biomarker score, with higher scores representing more dysfunction and inflammation, respectively.

\section{Results}

After adjustment for sex, age, energy intake, glucose metabolism status, body mass index, current smoking, prior CVD, educational level and physical activity, both alcohol and red wine consumption, but none of the other food groups, were associated with a lower endothelial dysfunction circulating biomarker score and a greater FMD. The associations for FMD were, however, not statistically significant. Only red wine consumption was associated with a lower low-grade inflammation circulating biomarker score. Results were independent of each of the other food groups.

\section{Conclusion}

Alcohol and red wine consumption may favourably influence processes involved in atherothrombosis. 


\section{Introduction}

A healthy diet, rich in fruit, vegetables and fish, low in dairy products and with moderate alcohol and red wine consumption is associated with a reduced incidence of cardiovascular disease (CVD). ${ }^{1-3}$ Endothelial dysfunction and low-grade inflammation are key phenomena in the pathobiology of $\mathrm{CVD}^{4-8}$ and seem to be influenced by dietary intake to the extent that a healthy diet is associated with less endothelial dysfunction and less low-grade inflammation. ${ }^{9-11}$ A healthy diet, therefore, could potentially reduce the incidence of CVD through these mechanisms.

Previous population-based studies on the association between diet and circulating biomarkers of endothelial dysfunction ${ }^{9-13}$ and circulating biomarkers of low-grade inflammation ${ }^{9-14}$ did not examine the food components of a healthy diet in relation to each other. The latter hampers direct comparison of these studies ${ }^{15}$, while data on the association between diet and flow-mediated vasodilation (FMD) of the brachial artery, an estimate of endothelium-dependent vasodilator function, are scarce. ${ }^{16,17}$

We therefore examined, in order to investigate in detail the associations between a healthy diet and endothelial dysfunction and low-grade inflammation, the associations between, on the one hand, fruit, vegetables, fish, dairy products, alcohol and red wine consumption and, on the other, five circulating biomarkers of endothelial dysfunction and FMD, and six circulating biomarkers of low-grade inflammation. In addition, we investigated whether any such associations were independent of lifestyle risk factors and each of the other food components of a healthy diet. Although the results will focus on (if any) independent associations, we systematically report on each of the abovementioned food groups in a large population-based cohort study of elderly individuals, situated in the Netherlands.

\section{Methods}

\section{Study population}

For the present study data were used from the 2000 Hoorn Study follow-up examination, described elsewhere. ${ }^{18-21}$ Of the original 822 participants, 20 were without dietary and biomarker data and one was without all covariate data (by participating only partially in the first study visit only). ${ }^{18}$ Of the remaining 801 participants, 63 were without circulating biomarker data ${ }^{21}$ and 158 were without FMD data. ${ }^{20}$ Thus, for the present analyses full data on diet and circulating biomarkers were available in 738 participants ${ }^{21}$ and full data on diet and FMD were available in 643 participants. ${ }^{20}$ 


\section{Dietary intake}

Diet was assessed by a validated self-administered Food Frequency Questionnaire (FFQ) originally developed for Dutch participants of the EPIC study. ${ }^{22-26}$ The FFQ queried participants to report habitual diet over the previous year. ${ }^{22,23}$ Energy intake and the nutrient compound ethanol were calculated with the help of the extended Dutch food composition database from 1996 (NEVO). ${ }^{27}$

Alcohol consumption was categorized as follows: non-consumers (no consumption of alcohol-containing beverages); moderate consumers (men: ethanol intake $>0$ and $\leq 20 \mathrm{~g} / \mathrm{d}$; women: ethanol intake $>0$ and $\leq 10 \mathrm{~g} / \mathrm{d}$ ) and high consumers (men: ethanol intake $>20 \mathrm{~g} / \mathrm{d}$; women: ethanol intake $>10 \mathrm{~g} / \mathrm{d}$ ). This allows for the possibility to investigate a U-shape association (if any) ${ }^{26}$, although alcohol consumption overall in the study population was relatively low and therefore a linear association can also be assumed. $^{28}$

As it remains unclear to what extent alcohol itself (i.e. ethanol) or any additional nutrient components such as polyphenols and flavonoids as present in red wine ${ }^{29}$ determine the association between alcohol and, endothelial dysfunction and lowgrade inflammation ${ }^{30-33}$, red wine was additionally investigated. Red wine consumption was dichotomized into non-consumers (no consumption of red wine) and consumers [red wine consumption; median (IQR) 28.6 (5.5-71.4) $\mathrm{ml} / \mathrm{d}$ ]. Then, the analyses were repeated for red wine, and the associations for alcohol and red wine were mutually adjusted for each other.

Based on the dietary data the following four food groups were constructed: vegetables, fruit, fish and dairy products. For the purpose of comparison to alcohol and red wine consumption, participants were categorized into tertiles according to the level of food group intake.

\section{Circulating biomarkers of endothelial dysfunction and low-grade inflammation}

Circulating biomarkers were quantified in serum samples by a multi-array detection system based on electrochemiluminescence technology (SECTOR Imager 2400, MesoScaleDiscovery, Gaithersburg, MD, USA) as described elsewhere. ${ }^{21,34,35}$ Briefly, soluble intercellular adhesion molecule 1 (sICAM-1), soluble vascular cell adhesion molecule 1 (sVCAM-1), soluble endothelial selectin (sE-selectin) and soluble thrombomodulin (STM) were determined as circulating biomarkers of endothelial dysfunction; and C-reactive protein (CRP), serum amyloid A (SAA), interleukin 6 (IL-6), interleukin 8 (IL-8), tumour necrosis factor $\alpha$ (TNF- $\alpha$ ) and SICAM-1 were determined as circulating biomarkers of low-grade inflammation. All serum samples were measured on a single production lot of multi-array plates and in duplication on separate designated wells on the same array plate. Intra- and inter-assay CV were for sICAM-1, 2.4 and 4.9\%; for sVCAM-1, 2.8 and 5.6\%; for sE-selectin, 2.6 and $6.7 \%$; for 
STM, 2.1 and 6.9\%; for CRP, 2.8 and 4.0\%; for SAA, 2.7 and 11.6\%; for IL-6, 5.6 and 13.0\%; for IL-8, 5.6 and $12.2 \%$ and for TNF- $\alpha, 3.9$ and $8.8 \%$, respectively.

In addition, von Willebrand factor (vWf), a circulating biomarker of endothelial dysfunction, was determined in citrated plasma by means of ELISA as described elsewhere. ${ }^{21}$ Intra- and inter-assay CV were for vWf, 3.4 and $7.9 \%$, respectively.

\section{Flow-mediated vasodilation}

The measurement of FMD was executed in accordance with international guidelines ${ }^{36,37}$ as described previously. ${ }^{20}$ This method allows for the determination of the peak arterial vasodilatory response (maximum diameter) after cuff-release ${ }^{20}$, which reflects endothelium-dependent vasodilation. ${ }^{38}$ Briefly, baseline diameter (mean of three measurements) was assessed with the use of an ultrasound scanner equipped with an 7.5 MHz linear probe (350 series, Pie Medical, Maastricht, The Netherlands) that was connected to a PC equipped with vessel wall movement detection software and an acquisition system (Wall Track System, Pie Medical, Maastricht, The Netherlands). Baseline peak flow velocity (mean of two measurements) was determined by pulsed-wave Doppler from a sample volume in the centre of the artery at a $60^{\circ}$ angle. A pressure cuff, placed on the forearm, was then automatically inflated and kept constant at supra-systolic pressure (SBP) (brachial SBP $+100 \mathrm{mmHg}$ ) in order to induce forearm ischaemia. After $5 \mathrm{~min}$ the cuff was released leading to an increase in blood flow and hereby in shear stress, which served as the stimulus for FMD response. Maximum peak flow velocity was measured within 15 seconds after the cuff release, and measures of arterial diameter were subsequently measured at $45,90,180$ and 300 seconds.

In some individuals measures of brachial diameter were missing at one $(n=70)$, two $(n=21)$, or three $(n=10)$ out of the four post cuff-release time points. For these individuals, we imputed the missing post cuff-release diameter(s) with a longitudinal regression method. ${ }^{39}$ Specifically, we regressed the repeated post cuff release measures of diameter available on baseline diameter and time with the use of generalized estimating equations. Because the relationship between time and diameter is not linear, time was treated as a categorical (dummy) variable to approximate the FMD response curve by estimation of the predicted diameter at each exact time point (i.e. at $45,90,180$ or 300 seconds after cuff release) based on the observed baseline and post cuff-release measures obtained. ${ }^{40}$ The predicted values hereby estimated were used to replace the missing value(s). FMD (peak arterial vasodilatory response, i.e. change in $\mathrm{mm}$ ) was then calculated as maximum postocclusion diameter minus baseline diameter and was used in the analyses as main dependent variable. 


\section{Other measurements}

Health status, medical history, glucose metabolism status, medication use, educational level, physical activity and smoking habits were assessed by questionnaire. Height, weight, serum creatinine, albuminuria, total and high-density lipoprotein (HDL) cholesterol, blood pressure, body mass index (BMI) $\left(\mathrm{kg} / \mathrm{m}^{2}\right)$ and prior CVD were determined as described elsewhere. ${ }^{18-21}$ Educational level was classified into six categories; primary education only; low level of secondary education; high level of secondary education; and tertiary education, either a bachelor degree, master degree, or PhD. Physical activity was expressed in metabolic equivalents (METs) (hour/wk), based on the amount of daily activities, including sports, bicycling, gardening, walking, doing odd jobs and housekeeping. Smoking behaviour was dichotomized into current smokers (15.1\%) and non-smokers, which included both previous smokers (45.5\%) and never smokers (39.4\%). Estimated glomerular filtration rate (eGFR) $\left(\mathrm{ml} / \mathrm{min} / 1.73 \mathrm{~m}^{2}\right)$ was calculated according to Levey's [Chronic Kidney Disease Epidemiology Collaboration (CKD-epi)] formula. ${ }^{41}$

\section{Statistical analyses}

Data analyses were performed with SPSS (Statistical Package for Social Sciences, version 20, IBM Corp., USA). Data were presented as mean ( $\pm S D)$, as median (interquartile range) for skewed variables or as percentages and as regression coefficients $(\beta)$ with their $95 \%$ confidence intervals $(95 \% \mathrm{Cl})$. Variables with a skewed distribution were natural log-transformed in order to meet normality criteria (serum triglycerides, METs, CRP, SAA, IL-6 and IL-8).

\section{Endothelial dysfunction}

For reasons of statistical efficiency and to reduce the influence of the biological variability of each measure, a circulating biomarker score for endothelial dysfunction was constructed using Z-scores as previously described elsewhere. ${ }^{21,34,42-44}$ The endothelial dysfunction circulating biomarker score consisted of VWf, sE-selectin, sTM, sVCAM-1 and SICAM-1, with a higher score indicating worse function.

With regard to FMD, the peak arterial vasodilatory response (i.e. the change in $\mathrm{mm}$ ) was used as outcome variable (lower FMD indicating worse function).

\section{Low-grade inflammation}

The low-grade inflammation circulating biomarker score consisted of CRP, SAA, IL-6, IL-8, TNF- $\alpha$ and SICAM-1 (sICAM-1 was included in both circulating biomarker scores as it is expressed by both monocytes and the endothelium) $)^{21,34,42-45}$, with a higher score indicating more inflammation.

First, we investigated the associations between, on the one hand, categories of alcohol consumption and tertiles of vegetables, fruit, fish and dairy product 
consumption, and, on the other hand, the endothelial dysfunction circulating biomarker score and FMD. Second, we investigated the associations between the above food groups and the low-grade inflammation circulating biomarker score. These analyses were adjusted for sex, age, glucose metabolism status and energy intake. For FMD the analyses were additionally adjusted for baseline diameter and flow increase (models 1). These associations were then additionally adjusted for BMI, current smoking, prior CVD, educational level and physical activity (models 2); and for each of the other food components of a healthy diet (i.e. fruit, vegetables, fish, dairy products and/or alcohol) (models 3). Although the results section will primarily focus on the independent associations (i.e. for alcohol and red wine consumption), we systematically report results on each of the food groups.

Thirdly, we repeated the above analyses for red wine consumption (models 1 to 3). Next, the associations for alcohol (i.e. the nutrient compound ethanol) and red wine consumption were mutually adjusted for each other.

Finally, as the presence of clinical disease may modify effects of diet on CVD ${ }^{44,46}$, we used interaction terms between clinical disease and food consumption to investigate any such effects. In the present study, clinical disease was defined as the presence of either CVD ( $n=242)$, type 2 diabetes mellitus ( $n=137)$, or both $(n=184)$.

A two-sided $p$-value $<0.05$ and for interaction $<0.10$ were considered statistically significant.

\section{Results}

\section{Study population}

Ninety-one percent of the 801 participants were $>60$ (range 50-87) years of age. Results focus on alcohol consumption and 139 individuals were classified as nonconsumers (34 men and 105 women: no consumption of alcohol-containing beverages), 414 were classified as moderate consumers (237 men: ethanol intake $>0$ and $\leq 20 \mathrm{~g} / \mathrm{d}$; 177 women: ethanol intake $>0$ and $\leq 10 \mathrm{~g} / \mathrm{d}$ ), and 248 were classified as high consumers (131 men: ethanol intake $>20 \mathrm{~g} / \mathrm{d}$; 117 women: ethanol intake $>10 \mathrm{~g} / \mathrm{d}$ ).

Age, the ratio of women to men, the prevalence of type 2 diabetes mellitus, a low educational level and the use of anti-hypertensive medication decreased over the categories of alcohol consumption, whereas current smoking, physical activity, eGFR and HDL cholesterol (Table 5.1), and the intake of energy, red wine, vegetables and fish increased over the categories of alcohol consumption (Table 5.2).

The endothelial dysfunction circulating biomarker score (i.e. the peak diameter change) decreased over the categories of alcohol consumption, whereas FMD increased. The low-grade inflammation circulating biomarker score also decreased over the categories of alcohol consumption (Table 5.2). 
Table 5.1 Population characteristics according to categories of alcohol consumption.

\begin{tabular}{|c|c|c|c|c|}
\hline \multirow[t]{2}{*}{ Characteristics } & \multicolumn{3}{|c|}{ Categories of alcohol consumption } & \multirow[t]{2}{*}{ p-trend } \\
\hline & none & moderate & high & \\
\hline Age, years & $70.7 \pm 7.1$ & $68.8 \pm 7.2$ & $66.8 \pm 6.9$ & $<0.001$ \\
\hline Sex, men / women & $34 / 105$ & $237 / 177$ & $131 / 117$ & $<0.001$ \\
\hline \multicolumn{5}{|l|}{ Glucose metabolism status * } \\
\hline Normal glucose metabolism, \% & 23.9 & 40.4 & 36.9 & \\
\hline Impaired glucose metabolism, $\%$ & 18.1 & 23.8 & 24.1 & \\
\hline Diabetes mellitus type $2, \%$ & 58.0 & 35.8 & 39.0 & 0.006 \\
\hline Body mass index, $\mathrm{kg} / \mathrm{m}^{2}$ & $28.1 \pm 5.0$ & $27.5 \pm 4.1$ & $27.8 \pm 3.8$ & 0.591 \\
\hline Current smoking, \% & 12.9 & 13.3 & 19.4 & 0.048 \\
\hline Prior cardiovascular disease, $\%$ & 62.8 & 52.2 & 52.7 & 0.105 \\
\hline Low-educational level, \% & 41.3 & 26.5 & 19.8 & $<0.001$ \\
\hline Physical activity, METs hour/wk & $49(21-99)$ & $81(47-129)$ & $85(51-132)$ & $<0.001$ \\
\hline Systolic blood pressure, $\mathrm{mmHg}$ & $142 \pm 22$ & $142 \pm 20$ & $142 \pm 20$ & 0.651 \\
\hline Diastolic blood pressure, $\mathrm{mmHg}$ & $83 \pm 11$ & $83 \pm 11$ & $84 \pm 11$ & 0.378 \\
\hline Anti-hypertensive medication, \% & 51.8 & 37.0 & 35.9 & 0.006 \\
\hline Total cholesterol, mmol/l & $5.7 \pm 1.1$ & $5.6 \pm 1.1$ & $5.9 \pm 0.9$ & 0.051 \\
\hline HDL cholesterol, mmol/l & $1.3 \pm 0.4$ & $1.3 \pm 0.4$ & $1.5 \pm 0.4$ & $<0.001$ \\
\hline LDL cholesterol, mmol// & $3.6 \pm 1.0$ & $3.6 \pm 0.9$ & $3.6 \pm 0.8$ & 0.585 \\
\hline Triglycerides, mmol/l & $1.5(1.1-2.2)$ & $1.3(1.0-1.8)$ & $1.3(1.0-2.0)$ & 0.838 \\
\hline Lipid-lowering medication, \% & 18.0 & 15.9 & 17.7 & 0.931 \\
\hline $\begin{array}{l}\text { Albuminuria [albumin/creatinine ratio }>2 \\
(\mathrm{mg} / \mathrm{mmol})], \%\end{array}$ & 15.1 & 16.3 & 13.8 & 0.624 \\
\hline Serum creatinine, $\mu \mathrm{mol} / \mathrm{l}$ & $91.6 \pm 17.1$ & $96.4 \pm 17.7$ & $95.6 \pm 16.1$ & 0.083 \\
\hline eGFR, $\mathrm{ml} / \mathrm{min} / 1.73 \mathrm{~m}^{2}$ & $60.9 \pm 12.4$ & $63.5 \pm 11.2$ & $64.2 \pm 11.1$ & 0.014 \\
\hline
\end{tabular}

Data are means \pm SD, median (interquartile range) or percentages, as appropriate, according to the following categories of alcohol consumption: non-consumers $(n=139)$; moderate consumers (men: ethanol intake 0-20 g/d; women: ethanol intake 0-10 g/d; $\mathrm{n}=414$ ); and high consumers (men: ethanol intake $>20 \mathrm{~g} / \mathrm{d}$; women: ethanol intake $>10 \mathrm{~g} / \mathrm{d}$; $\mathrm{n}=248$ ); $\mathrm{p}$-trend by linear regression analysis for continuous variables and by chi-square test for proportions. * normal glucose metabolism: fasting plasma glucose $<6.1 \mathrm{mmol} / \mathrm{l}$ and 2-hour post-load plasma glucose $<7.8 \mathrm{mmol} / \mathrm{l}$; impaired glucose metabolism: impaired fasting plasma glucose between $6.1 \mathrm{mmol} / \mathrm{l}$ and $7.0 \mathrm{mmol} / \mathrm{l}$ and impaired glucose tolerance defined as a 2-hour post-load plasma glucose between 7.8 and $11.1 \mathrm{mmol} / \mathrm{l}$; diabetes mellitus type 2: fasting plasma glucose $\geq 7.0 \mathrm{mmol} / \mathrm{l}$ and/or 2-hour post-load plasma glucose $\geq 11.1 \mathrm{mmol} / \mathrm{l}$; METs, metabolic equivalents; $\mathrm{HDL}$, high-density lipoprotein; LDL, low-density lipoprotein; eGFR, estimated glomerular filtration rate according to Levey's [Chronic Kidney Disease Epidemiology Collaboration (CKD-epi)] formula for the following four strata (only Caucasians present in the Hoorn study population); (1) women, serum creatinine $\leq 62 \mu \mathrm{mol} / \mathrm{l}$ : eGFR $=144 \times(\text { serum creatinine } / 0.7)^{-0.329} \times(0.993)^{\text {Age }} ;(2)$ women, serum creatinine $>62 \mu \mathrm{mol} / \mathrm{l}$ : eGFR $=144 \times(\text { serum creatinine } / 0.7)^{-1.209} \times(0.993)^{\text {Age }} ;$ (3) men, serum creatinine $\leq 80 \mu \mathrm{mol} / \mathrm{l}$ : eGFR $=141 \times$ (serum creatinine $/ 0.9)^{-0.411} \times(0.993)^{\mathrm{Age}} ;$ (4) men, serum creatinine $>80 \mu \mathrm{mol} / \mathrm{I}$ : eGFR $=141 \times($ serum creatinine $/ 0.9)^{-1.209} \times(0.993)^{\mathrm{Age}} \cdot 37$

As compared to the participants with data, those without either circulating biomarker $(n=63)$ or FMD $(n=158)$ data had a higher BMI and lower educational level, and used anti-hypertensive medication more frequently ( $p$-values <0.05). In addition, those without circulating biomarker data were younger and had type 2 diabetes mellitus more frequently, whereas those without FMD data were older and less physically active, had a lower eGFR and consumed alcohol and red wine less frequently ( $p$-values 
$<0.05$, other data not shown). The presence of clinical disease in participants with data was $70 \%$.

Table 5.2 Diet, endothelial dysfunction and low-grade inflammation according to categories of alcohol consumption.

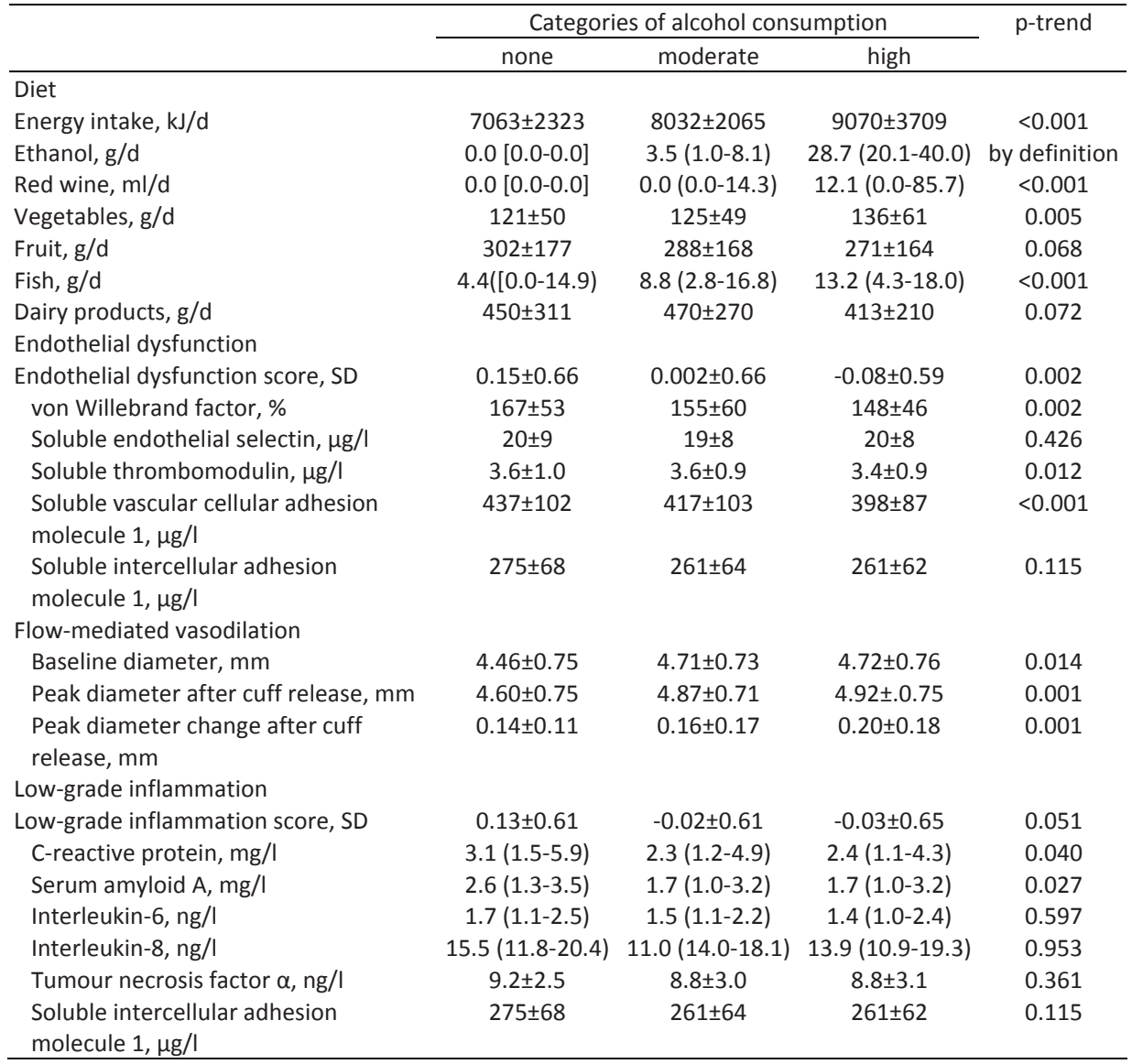

Data are means \pm SD or median (interquartile range), as appropriate, according to the following categories of alcohol consumption: non-consumers; moderate consumers (men: ethanol intake 0-20 g/d; women: ethanol intake 0-10 g/d); and high consumers (men: ethanol intake $>20 \mathrm{~g} / \mathrm{d}$; women: ethanol intake $>10 \mathrm{~g} / \mathrm{d}$ ); $\mathrm{p}$-trend by linear regression analyses.

\section{Associations between food consumption and endothelial dysfunction}

\section{Alcohol consumption}

After adjustment for sex, age, glucose metabolism status and energy intake, moderate and high consumers had a lower endothelial dysfunction circulating biomarker score $[\beta(95 \% \mathrm{Cl})-0.16(-0.37 ; 0.04)$ and $-0.23(-0.45 ;-0.01)$, respectively (Table 5.3 , model 1$)]$ 
as compared to non-consumers (reference group; $\mathrm{p}$-trend $=0.051)$. The results showed a positive association for FMD that was not statistically significant $[(0.01(-0.21 ; 0.23)$ and $0.16(-0.08 ; 0.39)$, respectively (Table 5.3, model 1)]. Additional adjustment for $\mathrm{BMI}$, current smoking, prior CVD, educational level, physical activity and each of the other food components of a healthy diet did not materially change the results (Table 5.3, models 2-3).

Vegetable, fruit, fish, and dairy product consumption were neither associated with the endothelial dysfunction circulating biomarker score nor with FMD (please see Appendix 5, Table S5.1).

\section{Red wine consumption}

After adjustment for sex, age, glucose metabolism status and energy intake, red wine consumers [ $n=365$; median (IQR) intake, $28.6(5.5-71.4) \mathrm{ml} / \mathrm{d}$ ] had a lower endothelial dysfunction circulating biomarker score $[-0.21(-0.35 ;-0.07)$ (Table 5.3, model 1)] as compared to non-consumers (reference group). The results showed a positive association for FMD that was not statistically significant $[0.08(-0.07 ; 0.23)$ (Table 5.3, model 1)]. Additional adjustment for BMI, current smoking, prior CVD, educational level, physical activity and each of the other food components of a healthy diet did not materially change the results (Table 5.3, models 2-3).

\section{Mutual adjustment}

The association between alcohol consumption and the endothelial dysfunction circulating biomarker score (Table 5.3, model 2) was attenuated after additional adjustment for red wine consumption (Table 5.3, model 4a).

The association between red wine consumption and the endothelial dysfunction circulating biomarker score (Table 5.3, model 2) did not materially change after additional adjustment for the nutrient compound ethanol (Table 5.3, model 4b).

\section{Associations between food consumption and low-grade inflammation}

Alcohol, vegetable, fruit, fish and dairy product consumption were not associated with the low-grade inflammation circulating biomarker score (Table 5.3 and please see Appendix 5, Table S5.1). 


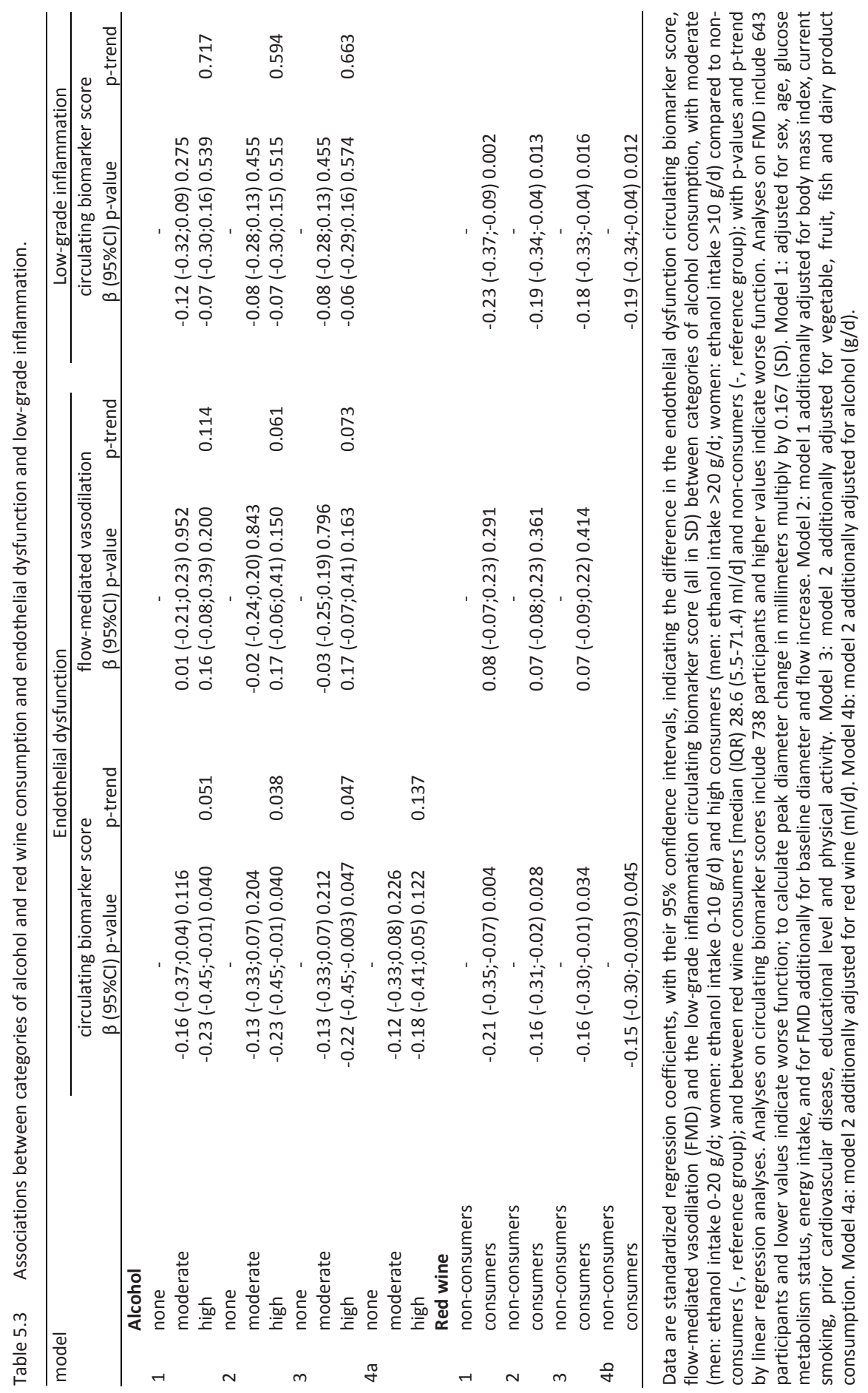




\section{Red wine consumption}

After adjustment for sex, age, glucose metabolism status and energy intake, red wine consumers had a lower low-grade inflammation circulating biomarker score [-0.23 $(-0.37 ;-0.09)$ (Table 5.3, model 1)] as compared to non-consumers (reference group). Additional adjustment for BMI, current smoking, prior CVD, educational level, physical activity and each of the other food components of a healthy diet did not materially change the results (Table 5.3, models 2-3).

\section{Mutual adjustment}

The association between red wine consumption and the low-grade inflammation circulating biomarker score (Table 5.3, model 2) did not materially change after additional adjustment for the nutrient compound ethanol (Table 5.3, model 4b).

\section{Results according to the presence or absence of clinical disease}

The association between diet and endothelial dysfunction and low-grade inflammation may differ according to the presence of clinical disease. ${ }^{44,46}$ In particular, it has been suggested that the consumption of red wine may be favourable in $\mathrm{CVD}^{30-33}$, whereas the consumption of dairy products may have adverse effects. ${ }^{47}$ If we tested these hypotheses the results showed that the consumption of red wine was inversely associated with the low-grade inflammation circulation biomarker score in participants with clinical disease (please see Appendix 5, Table S5.2; $P_{\text {interaction }}=0.098$ ). In addition, the results for dairy products showed that the consumption of dairy products was inversely associated with the endothelial dysfunction circulating biomarker score in participants with clinical disease (please see Appendix 5, Table S5.2; $P_{\text {interaction }}=0.071$ ).

As the latter result appeared in contrast to our hypothesis, by suggesting a beneficial effect of dairy product consumption on endothelial dysfunction, we repeated the stratified analyses for the following components of total dairy products: cheese, low$(<2.0 \%)$ and high- $(\geq 2.0 \%)$ fat dairy products. Although not statistically significant, the results showed an inverse association for low-fat dairy production consumption in participants with clinical disease as opposed to a positive association for high-fat dairy products in participants without clinical disease (please see Appendix 5, Table S5.2).

\section{Additional analyses}

Additional adjustment for total and HDL cholesterol, systolic blood pressure, the use of lipid-lowering and/or anti-hypertensive medication, in addition to eGFR, did not materially change the results (data not shown) 
When we re-analyzed the data with adjustment for both current and previous smokers (separate dummy variables) the results did not materially change (data not shown).

When we re-analyzed the data for red wine and the individual biomarkers of the endothelial dysfunction circulating biomarker score, the results showed inverse associations for each individual biomarker, except for sE-selectin (Figure 5.1A). When we re-analyzed the data for red wine and the individual biomarkers of the low-grade inflammation circulating biomarker score, the results showed inverse associations for each individual biomarker (Figure 5.1B).

\section{endothelial dysfunction}

A

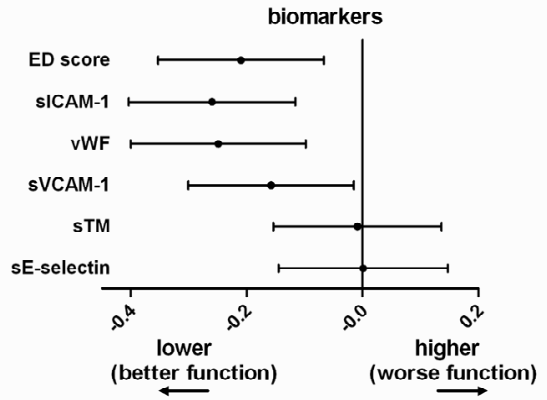

low-grade inflammation

B

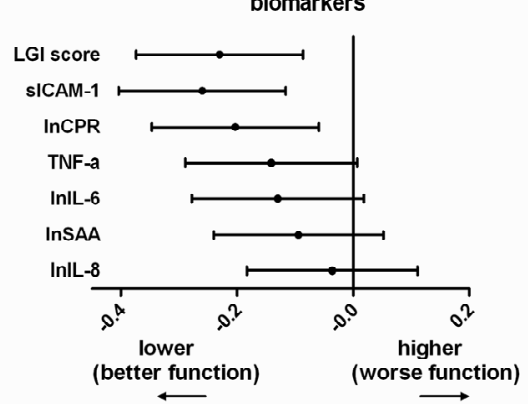

Figure 5.1 Endothelial dysfunction and low-grade inflammation for red wine consumers, compared to non-consumers, with endothelial dysfunction circulating biomarkers (A) and low-grade inflammation circulating biomarkers (B). Dots show each of the individual associations, with whiskers indicating their 95\% confidence intervals. Higher circulating biomarkers indicate worse function and more inflammation. Results are adjusted for sex, age, glucose metabolism status, energy intake, body mass index, current smoking, prior cardiovascular disease, educational level and physical activity. ED, endothelial dysfunction circulating biomarker score; vWf, von Willebrand factor; sICAM-1, soluble intercellular adhesion molecule 1; sVCAM-1, soluble vascular cell adhesion molecule 1; STM, soluble thrombomodulin; sE-selectin, soluble endothelial selection; LGI, low-grade inflammation circulating biomarker score; TNF-a, tumour necrosis factor alpha; In, natural log normalized; CRP, C-reactive protein; IL-6, interleukin-6; SAA, serum amyloid A; IL-8, interleukin 8.

Results did not materially change when trends were tested using the median intake per category of alcohol consumption. Alternative analyses with red wine consumers divided into moderate consumers (red wine intake: $>0$ and $\leq 75 \mathrm{ml} / \mathrm{d}$ ) and high consumers (red wine intake: $>75 \mathrm{ml} / \mathrm{d}$ ) gave similar results $(\mathrm{p}$-trend $=0.034$ for the endothelial dysfunction circulating biomarker score and $p$-trend $=0.028$ for the lowgrade inflammation circulating biomarker score, data not shown). The Pearson's correlation coefficient between alcohol and red wine was $r=0.34$. When we performed 
mutual adjustments for red wine and alcohol with categorical data only the results did not materially change.

Models for FMD without adjustment for baseline diameter gave similar results. When we restricted the analyses for FMD to the subset of participants without missing postocclusion diameters, re-analyses of the data also gave similar results (data not shown).

Missing covariate data were replaced by the population's mean. When we restricted the analyses to the subset of participants with full covariate data, re-analyses of the data gave similar results (data not shown).

\section{Discussion}

The present population-based cohort study is the first to evaluate in relation to each other the associations between fruit, vegetables, fish, dairy product, alcohol and red wine consumption and endothelial dysfunction (determined by a set of five circulating biomarkers and FMD), in addition to low-grade inflammation (determined by a set of six circulating biomarkers).

The study had two main findings. First, after adjustment for sex, age, energy intake, glucose metabolism status, BMI, current smoking, prior CVD, educational level and physical activity, both alcohol and red wine consumption were associated with a lower endothelial dysfunction circulating biomarker score and a greater FMD. The associations for FMD were, however, not statistically significant. Importantly, these results were independent of each of the other food components of a healthy diet. Second, after adjustment for sex, age, energy intake, glucose metabolism status, BMI, current smoking, prior CVD, educational level and physical activity, none of the food groups showed an association with the low-grade inflammation circulating biomarker score, except for the consumption of red wine. Red wine consumption was associated with a lower low-grade inflammation circulating biomarker score. This association was independent of each of the other food components of a healthy diet and was strongest in participants with clinical disease.

The present study expands previous knowledge on the associations between diet and endothelial dysfunction and low-grade inflammation ${ }^{9-17}$ as it showed that in particular alcohol-containing beverage consumption (i.e. alcohol and red wine) was independently associated with less endothelial dysfunction and less low-grade inflammation. In addition, the fact that the association for red wine and low-grade inflammation was stronger in participants with diabetes and CVD may support the view that dietary recommendations have to be intensified in these patients. ${ }^{46}$ However, a role for the other food components of a healthy diet on endothelial dysfunction and low-grade inflammation was less clear.

In particular, the result for total dairy product consumption in participants with clinical disease appeared to oppose our hypothesis and as we cannot rule out the play 
of chance it should be interpreted with caution. Nevertheless, additional analyses with components of total dairy products suggested that more low-fat dairy product consumption might be beneficial in diabetes and CVD, whereas more high-fat dairy product consumption could have adverse effects in participants free of clinical disease. Although this might explain how high-fat, but not low-fat, dairy product consumption was associated with CVD mortality ${ }^{25}$, which thereby expands previous knowledge on this topic ${ }^{24}$, these results were not statistically significant and further investigation of the role of dairy product consumption on endothelial dysfunction and low-grade inflammation is therefore warranted.

The vascular endothelium has many functions in a heterogeneous way throughout the vasculature. ${ }^{38,47,48}$ The extensive array of endothelium-derived circulating biomarkers and FMD (a measure of endothelium-dependent vasodilation) capture several dimensions of endothelial dysfunction. ${ }^{38,47,48}$ Indeed, both higher concentrations of the circulating biomarkers and a smaller FMD response are associated with (incident) cardiovascular disease..$^{5-8}$ The present results for alcohol and red wine showed that, in particular, high alcohol consumers and red wine consumers had a lower endothelial dysfunction circulating biomarker score and greater FMD, suggesting a favourable effect on CVD. The results for FMD were, however, not statistically significant. This might be explained by the fact that 158 participants missed FMD data due to a greater body mass index ${ }^{20}$, which by itself is associated with a less healthy diet ${ }^{49}$ and a smaller FMD response. ${ }^{20}$ Alternatively, this result suggests differential effects of dietary compounds on individual dimensions of endothelial dysfunction, as suggested by previous studies. $^{12,16,32}$

Additionally, it is unclear to what extent alcohol itself (i.e. the nutrient compound ethanol) or any additional nutrient components such as polyphenols and flavonoids may determine the association between alcohol and endothelial dysfunction and lowgrade inflammation. ${ }^{30-33}$ In the present study the association between alcohol consumption and the endothelial dysfunction circulating biomarker score was attenuated after adjustment for red wine, while the associations between red wine and the endothelial dysfunction and low-grade inflammation circulating biomarker scores did not materially change after adjustment for alcohol consumption. This suggests that red wine, rich in polyphenols and flavonoids ${ }^{29}$, has the ability to favourably modulate adhesion molecules and inflammatory mediators (i.e. circulating biomarkers) independent of its alcohol content ${ }^{51}$, while an association between alcohol and circulating biomarkers that is independent from red wine cannot be excluded.

The present study has some limitations. First, a survival bias could have affected the results, as it is likely that participants with an unhealthy diet, worse endothelial dysfunction and worse low-grade inflammation may have died before the start of the study. ${ }^{1-3,5-8}$ However, such a bias would likely have led to an underestimation of the reported associations. Second, we cannot exclude that the results are affected by residual confounding, although further additional adjustment for total and HDL 
cholesterol, systolic blood pressure, the use of lipid-lowering and/or anti-hypertensive medication and eGFR did not materially change the results. Finally, although we hypothesized that more vegetable, fruit and fish consumption were favourably associated with endothelial dysfunction and low-grade inflammation ${ }^{1-3}$, we cannot exclude that the variation in consumption between persons was too small to reveal any associations between these food groups and endothelial dysfunction and lowgrade inflammation. Alternatively, effects of (any of) these food groups might primarily be seen earlier in life. ${ }^{43}$

In conclusion, alcohol and red wine consumption were associated with lower circulating biomarkers of endothelial dysfunction and red wine consumption was associated with lower circulating biomarkers of low-grade inflammation, whereas results for vegetable, fruit, fish and dairy product consumption were less clear. Importantly, these results were independent of vegetable, fruit, fish and dairy product consumption. This suggests that food components of a healthy diet, and in particular alcohol-containing beverage consumption, may favourably influence endothelial dysfunction and low-grade inflammation. 


\section{References}

1. Knoops KT, de Groot LC, Kromhout D, Perrin AE, Moreiras-Varela O, Menotti A, van Staveren WA. Mediterranean diet, lifestyle factors, and 10-year mortality in elderly European men and women: the HALE project. JAMA. 2004;292:1433-1439.

2. Hu FB, Rimm EB, Stampfer MJ, Ascherio A, Spiegelman D, Willett WC. Prospective study of major dietary patterns and risk of coronary heart disease in men. Am J Clin Nutr 2000; 72: 912-921.

3. Mitrou PN, Kipnis V, Thiebaut AC, Reedy J, Subar AF, Wirfalt E, Flood A, Mouw T, Hollenbeck AR, Leitzmann MF, Schatzkin A. Mediterranean dietary pattern and prediction of all-cause mortality in a US population: results from the NIH-AARP Diet and Health Study. Arch Intern Med. 2007;167: 2461-2468.

4. Hansson GK. Inflammation, atherosclerosis, and coronary artery disease. N Engl J Med 2005; 352: 1685-1695.

5. Blankenberg S, Rupprecht HJ, Bickel C, Peetz D, Hafner G, Tiret L, Meyer J. Circulating cell adhesion molecules and death in patients with coronary artery disease. Circulation. 2001;104:1336-1342.

6. Ridker PM, Hennekens $\mathrm{CH}$, Buring JE, Rifai N. C-reactive protein and other markers of inflammation in the prediction of cardiovascular disease in women. N Engl J Med 2000; 342: 836-843.

7. Jager A, van Hinsbergh VW, Kostense PJ, Emeis JJ, Yudkin JS, Nijpels G, Dekker JM, Heine RJ, Bouter LM, Stehouwer CD. von Willebrand factor, C-reactive protein, and 5-year mortality in diabetic and nondiabetic subjects: the Hoorn Study. Arterioscler Thromb Vasc Biol. 1999;19:3071-3078.

8. Inaba Y, Chen JA, Bergmann SR. Prediction of future cardiovascular outcomes by flow-mediated vasodilatation of brachial artery: a meta-analysis. Int J Cardiovasc Imaging 2010; 26: 631-640.

9. Lopez-Garcia E, Schulze MB, Fung TT, Meigs JB, Rifai N, Manson JE, Hu FB. Major dietary patterns are related to plasma concentrations of markers of inflammation and endothelial dysfunction. Am J Clin Nutr. 2004;80:1029-1035.

10. Fung TT, McCullough ML, Newby PK, Manson JE, Meigs JB, Rifai N, Willett WC, Hu FB. Diet-quality scores and plasma concentrations of markers of inflammation and endothelial dysfunction. Am J Clin Nutr. 2005;82:163-173.

11. Nettleton JA, Steffen LM, Mayer-Davis EJ, Jenny NS, Jiang R, Herrington DM, Jacobs DR, Jr. Dietary patterns are associated with biochemical markers of inflammation and endothelial activation in the Multi-Ethnic Study of Atherosclerosis (MESA). Am J Clin Nutr. 2006;83:1369-1379.

12. He K, Liu K, Daviglus ML, Jenny NS, Mayer-Davis E, Jiang R, Steffen L, Siscovick D, Tsai M, Herrington D. Associations of dietary long-chain n-3 polyunsaturated fatty acids and fish with biomarkers of inflammation and endothelial activation (from the Multi-Ethnic Study of Atherosclerosis [MESA]). Am J Cardiol 2009; 103: 1238-1243.

13. Shai I, Rimm EB, Schulze MB, Rifai N, Stampfer MJ, Hu FB. Moderate alcohol intake and markers of inflammation and endothelial dysfunction among diabetic men. Diabetologia 2004; 47: 1760-1767.

14. Imhof A, Woodward M, Doering A, Helbecque N, Loewel H, Amouyel P, Lowe GD, Koenig W. Overall alcohol intake, beer, wine, and systemic markers of inflammation in western Europe: results from three MONICA samples (Augsburg, Glasgow, Lille). Eur Heart J 2004; 25: 2092-2100.

15. Calder PC, Ahluwalia N, Brouns F, Buetler T, Clement K, Cunningham K, Esposito K, Jönsson LS, Kolb H, Lansink M, Marcos A, Margioris A, Matusheski N, Nordmann H, O'Brien J, Pugliese G, Rizkalla S, Schalkwijk C, Tuomilehto J, Wärnberg J, Watzl B, Winklhofer-Roob BM. Dietary factors and low-grade inflammation in relation to overweight and obesity. Br J Nutr 2011; 106 Suppl 3: S5-78.

16. Anderson JS, Nettleton JA, Herrington DM, Johnson WC, Tsai MY, Siscovick D. Relation of omega-3 fatty acid and dietary fish intake with brachial artery flow-mediated vasodilation in the Multi-Ethnic Study of Atherosclerosis. Am J Clin Nutr 2010; 92: 1204-1213.

17. Suzuki K, Elkind MS, Boden-Albala B, Jin Z, Berry G, Di Tullio MR, Sacco RL, Homma S. Moderate alcohol consumption is associated with better endothelial function: a cross sectional study. BMC Cardiovasc Disord 2009; 9: 8.

18. Henry RM, Kostense PJ, Spijkerman AM, Dekker JM, Nijpels G, Heine RJ, Kamp O, Westerhof N, Bouter LM, Stehouwer CD; Hoorn Study. Arterial stiffness increases with deteriorating glucose tolerance status: the Hoorn Study. Circulation 2003; 107: 2089-2095. 
19. Du H, van der A DL, van Bakel MM, van der Kallen CJ, Blaak EE, van Greevenbroek MM, Jansen EH, Nijpels G, Stehouwer CD, Dekker JM, Feskens EJ. Glycemic index and glycemic load in relation to food and nutrient intake and metabolic risk factors in a Dutch population. Am J Clin Nutr 2008; 87: 655661.

20. Henry RM, Ferreira I, Kostense PJ, Dekker JM, Nijpels G, Heine RJ, Kamp O, Bouter LM, Stehouwer CD. Type 2 diabetes is associated with impaired endothelium-dependent, flow-mediated dilation, but impaired glucose metabolism is not; The Hoorn Study. Atherosclerosis 2004; 174: 49-56.

21. van Bussel BC, Henry RM, Schalkwijk CG, Dekker JM, Nijpels G, Stehouwer CD. Low-grade inflammation, but not endothelial dysfunction, is associated with greater carotid stiffness in the elderly: the Hoorn Study. J Hypertens 2012; 30: 744-752.

22. Ocké MC, Bueno-de-Mesquita HB, Goddijn HE, Jansen A, Pols MA, van Staveren WA, Kromhout D. The Dutch EPIC food frequency questionnaire. I. Description of the questionnaire, and relative validity and reproducibility for food groups. Int J Epidemiol 1997; 26 Suppl 1: S37-48.

23. Ocke MC, Bueno-de-Mesquita HB, Pols MA, Smit HA, van Staveren WA, Kromhout D. The Dutch EPIC food frequency questionnaire. II. Relative validity and reproducibility for nutrients. Int J Epidemiol 1997; 26 Suppl 1: S49-58.

24. Snijder MB, van der Heijden AA, van Dam RM, Stehouwer CD, Hiddink GJ, Nijpels G, Heine RJ, Bouter LM, Dekker JM. Is higher dairy consumption associated with lower body weight and fewer metabolic disturbances? The Hoorn Study. Am J Clin Nutr 2007; 85: 989-995.

25. van Aerde MA, Soedamah-Muthu SS, Geleijnse JM, Snijder MB, Nijpels G, Stehouwer CD, Dekker JM. Dairy intake in relation to cardiovascular disease mortality and all-cause mortality: the Hoorn Study. Eur J Nutr 2013;52:609-616.

26. de Vegt F, Dekker JM, Groeneveld WJ, Nijpels G, Stehouwer CD, Bouter LM, Heine RJ. Moderate alcohol consumption is associated with lower risk for incident diabetes and mortality: the Hoorn Study. Diabetes Res Clin Pract 2002; 57: 53-60.

27. NEVO, Nederlands voedingsstoffenbestand, NEVO-tabel 1996, The Hague, The Netherlands, NEVO 1996.

28. Koppes LL, Dekker JM, Hendriks HF, Bouter LM, Heine RJ. Moderate alcohol consumption lowers the risk of type 2 diabetes: a meta-analysis of prospective observational studies. Diabetes Care 2005; 28 : 719-725.

29. Manach C, Scalbert A, Morand C, Remesy C, Jimenez L. Polyphenols: food sources and bioavailability. Am J Clin Nutr 2004; 79: 727-747.

30. Nova E, Baccan GC, Veses A, Zapatera B, Marcos A. Potential health benefits of moderate alcohol consumption: current perspectives in research. Proc Nutr Soc 2012; 71: 307-315.

31. Bau PF, Bau CH, Rosito GA, Manfroi WC, Fuchs FD. Alcohol consumption, cardiovascular health, and endothelial function markers. Alcohol 2007; 41: 479-488.

32. Schini-Kerth VB, Auger C, Kim JH, Etienne-Selloum N, Chataigneau T. Nutritional improvement of the endothelial control of vascular tone by polyphenols: role of NO and EDHF. Pflugers Arch 2010; 459: 853-862.

33. Gresele P, Cerletti C, Guglielmini G, Pignatelli P, de Gaetano G, Violi F. Effects of resveratrol and other wine polyphenols on vascular function: an update. J Nutr Biochem 2011; 22: 201-211.

34. van Bussel BC, Schouten F, Henry RM, Schalkwijk CG, de Boer MR, Ferreira I, Smulders YM, Twisk JW, Stehouwer CD. Endothelial dysfunction and low-grade inflammation are associated with greater arterial stiffness over a 6-year period. Hypertension 2011; 58: 588-595.

35. van Bussel BC, Ferreira I, van de Waarenburg MP, van Greevenbroek MM, van der Kallen CJ, Henry RM, Feskens EJ, Stehouwer CD, Schalkwijk CG. Multiple Inflammatory Biomarker Detection in a Prospective Cohort Study: A Cross-Validation between Well-Established Single-Biomarker Techniques and an Electrochemiluminescense-Based Multi-Array Platform. PLoS One 2013; 8: e58576.

36. Thijssen DH, Black MA, Pyke KE, Padilla J, Atkinson G, Harris RA, Parker B, Widlansky ME, Tschakovsky $\mathrm{ME}$, Green DJ. Assessment of flow mediated dilation (FMD) in humans: a methodological and technical guideline. Am J Physiol Heart Circ Physiol 2011; 300: H2-12. 
37. Corretti MC, Anderson TJ, Benjamin EJ, Celermajer D, Charbonneau F, Creager MA, Deanfield J, Drexler H, Gerhard-Herman M, Herrington D, Vallance P, Vita J, Vogel R; International Brachial Artery Reactivity Task Force. Guidelines for the ultrasound assessment of endothelial-dependent flowmediated vasodilation of the brachial artery: a report of the International Brachial Artery Reactivity Task Force. J Am Coll Cardiol 2002; 39: 257-265.

38. Deanfield J, Donald A, Ferri C, Giannattasio C, Halcox J, Halligan S, Lerman A, Mancia G, Oliver JJ, Pessina AC, Rizzoni D, Rossi GP, Salvetti A, Schiffrin EL, Taddei S, Webb DJ; Working Group on Endothelin and Endothelial Factors of the European Society of Hypertension. Endothelial function and dysfunction. Part I: Methodological issues for assessment in the different vascular beds: a statement by the Working Group on Endothelin and Endothelial Factors of the European Society of Hypertension. J Hypertens 2005; 23: 7-17.

39. Twisk J, de Vente W. Attrition in longitudinal studies. How to deal with missing data. J Clin Epidemiol 2002; 55: 329-337.

40. Twisk JW. Applied Longitudinal Data Analysis for Epidemiology: A practical guide, Cambridge, United Kingdom, The Press syndicate of the University of Cambridge, 2003.

41. Levey AS, Stevens LA, Schmid CH, Zhang YL, Castro AF 3rd, Feldman HI, Kusek JW, Eggers P, Van Lente F, Greene T, Coresh J; CKD-EPI (Chronic Kidney Disease Epidemiology Collaboration). A new equation to estimate glomerular filtration rate. Ann Intern Med 2009; 150: 604-612.

42. Yudkin JS, Stehouwer CD, Emeis JJ, Coppack SW. C-reactive protein in healthy subjects: associations with obesity, insulin resistance, and endothelial dysfunction: a potential role for cytokines originating from adipose tissue? Arterioscler Thromb Vasc Biol 1999; 19: 972-978.

43. van Bussel BC, Henry RM, Schalkwijk CG, Ferreira I, Feskens EJ, Streppel MT, Smulders YM, Twisk JW, Stehouwer CD. Fish consumption in healthy adults is associated with decreased circulating biomarkers of endothelial dysfunction and inflammation during a 6-year follow-up. J Nutr 2011; 141: 1719-1725.

44. van Bussel BC, Soedamah-Muthu SS, Henry RM, Schalkwijk CG, Ferreira I, Chaturvedi N, Toeller M, Fuller JH, Stehouwer CD; EURODIAB Prospective Complications Study Group. Unhealthy dietary patterns associated with inflammation and endothelial dysfunction in type 1 diabetes: the EURODIAB study. Nutr Metab Cardiovasc Dis 2013; 23: 758-764.

45. Schram MT, Stehouwer CD. Endothelial dysfunction, cellular adhesion molecules and the metabolic syndrome. Horm Metab Res 2005; 37 Suppl 1: 49-55.

46. Lichtenstein AH, Appel $\amalg$, Brands $M$, Carnethon M, Daniels S, Franch HA, Franklin B, Kris-Etherton P, Harris WS, Howard B, Karanja N, Lefevre M, Rudel L, Sacks F, Van Horn L, Winston M, Wylie-Rosett J. Diet and lifestyle recommendations revision 2006: a scientific statement from the American Heart Association Nutrition Committee. Circulation 2006; 114: 82-96.

47. Aird WC. Phenotypic heterogeneity of the endothelium: I. Structure, function, and mechanisms. Circ Res 2007; 100: 158-173.

48. Aird WC. Phenotypic heterogeneity of the endothelium: II. Representative vascular beds. Circ Res 2007; 100: 174-190.

49. Mozaffarian D, Hao T, Rimm EB, Willett WC, Hu FB. Changes in diet and lifestyle and long-term weight gain in women and men. N Engl J Med 2011; 364: 2392-2404.

50. Sacanella E, Vázquez-Agell M, Mena MP, Antúnez E, Fernández-Solá J, Nicolás JM, Lamuela-Raventós RM, Ros E, Estruch R. Down-regulation of adhesion molecules and other inflammatory biomarkers after moderate wine consumption in healthy women: a randomized trial. Am J Clin Nutr 2007; 86: 1463-1469. 


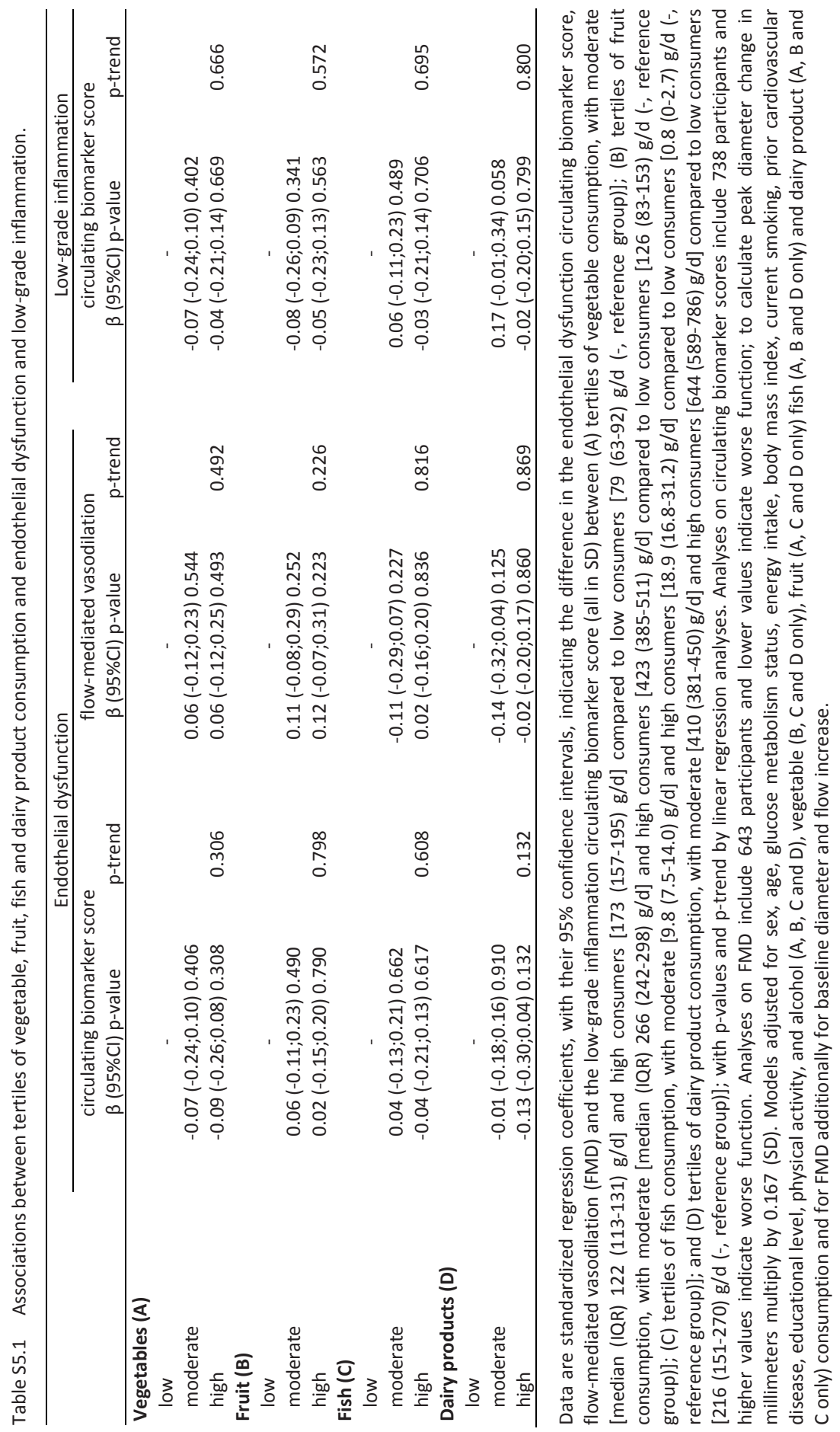




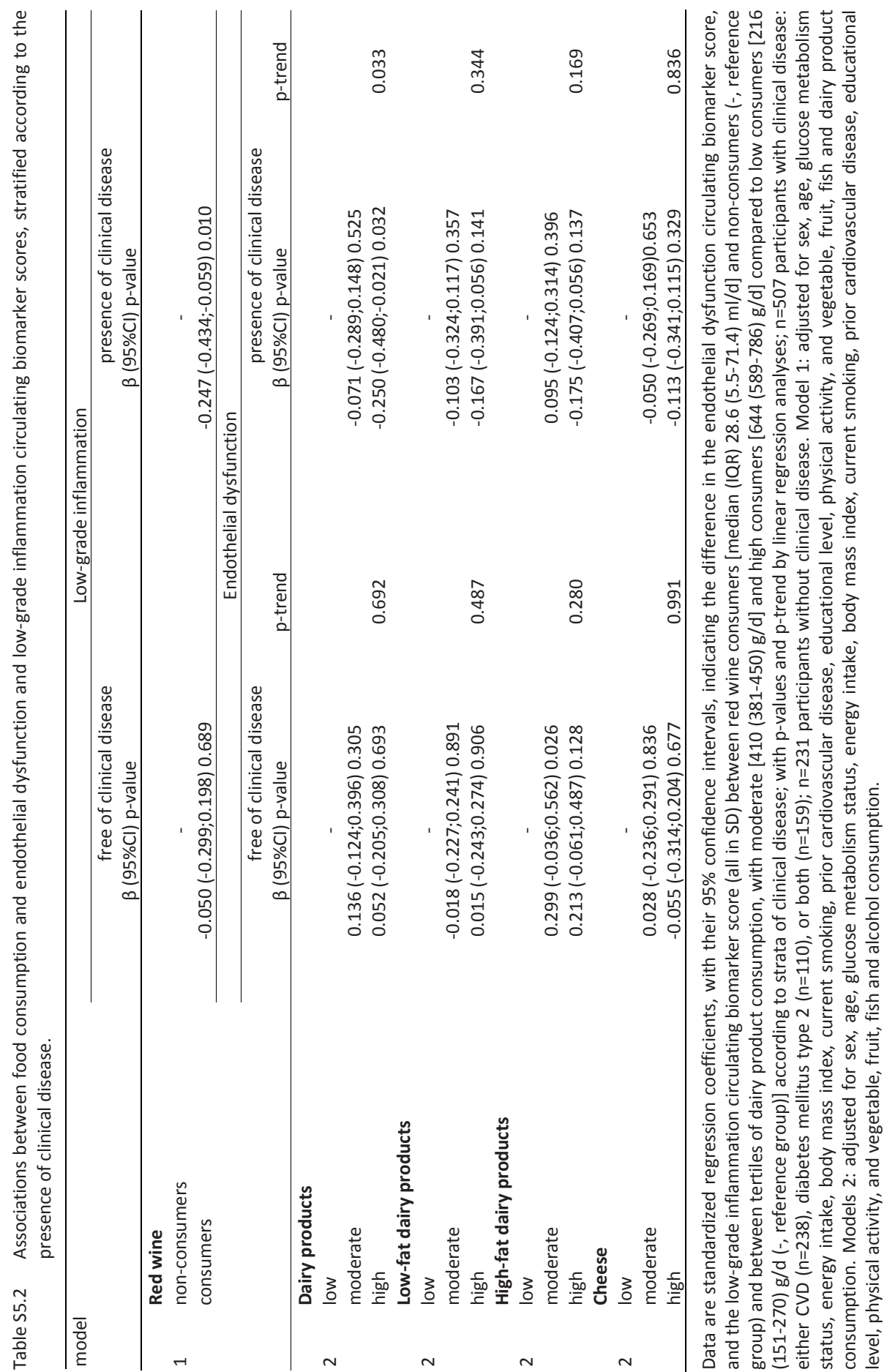




\section{Chapter \\ 6}

A healthy diet is associated with less endothelial dysfunction and less low-grade inflammation over a

7-year period - the CODAM study

BCT van Bussel, RMA Henry, I Ferreira, MMJ van Greevenbroek, CJH van der Kallen, JWR Twisk, EJM Feskens, CG Schalkwijk, CDA Stehouwer

Submitted for publication 


\section{Abstract}

\section{Background}

A healthy diet rich in fish, fruit and vegetables, but low in alcohol and dairy products, has been associated with less incident cardiovascular disease (CVD), but the mechanisms are unclear. Endothelial dysfunction and low-grade inflammation play important roles in the development of CVD. A healthy diet might modify these phenomena.

\section{Objective}

To investigate the association between the above food groups and overall biomarker scores of endothelial dysfunction and low-grade inflammation in a 7-year longitudinal study.

\section{Design}

In 557 participants with increased CVD risk, we determined diet by food frequency questionnaire and measured biomarkers of endothelial dysfunction [von Willebrand factor, soluble vascular cell adhesion molecule 1 , soluble endothelial selectin, soluble thrombomodulin, soluble intercellular adhesion molecule 1 (sICAM-1)] and of low-grade inflammation (C-reactive protein, serum amyloid A, interleukin 6, interleukin 8, tumour necrosis factor $\alpha$ and sICAM-1). Biomarkers were combined into overall scores (higher scores indicating worse function). Measurements were performed at baseline and after (median) 7 years. Longitudinal data were analyzed with generalized estimating equations and adjusted for sex, age, glucose metabolism status, energy intake, body mass index, physical activity, alcohol consumption and smoking status.

\section{Results}

Higher consumption of fish (per $100 \mathrm{~g} / \mathrm{wk}$ ), but not vegetables, fruit, alcohol-containing beverages or dairy products, was associated with a lower overall endothelial dysfunction score over 7 years: $\beta(95 \% \mathrm{Cl})-0.027(-0.051 ;-0.004)$. No associations were observed with the overall low-grade inflammation score. Further food component analyses indicated that consumption of more lean fish and raw vegetables, and less high-fat dairy products was associated with less endothelial dysfunction. Consumption of more fresh fruit and wine, and less high-fat dairy products was associated with less low-grade inflammation.

\section{Conclusions}

These data suggest that dietary modification of endothelial dysfunction and low-grade inflammation, processes that are important in atherothrombosis, is possible. 


\section{Introduction}

A healthy diet (i.e. a diet rich in fish, fruit and vegetables, but low in alcohol and dairy products) has been associated with less incident cardiovascular disease (CVD). ${ }^{1-3}$ Endothelial dysfunction and low-grade inflammation play important roles in the development of $C V D^{4-9}$ and dietary modification of these phenomena may constitute a mechanism through which CVD may be prevented. ${ }^{10}$

However, population-based studies that investigated the association between a healthy diet on the one hand and endothelial dysfunction and low-grade inflammation $^{11-17}$ on the other were primarily cross-sectional in nature ${ }^{11-17}$ and focussed on either overall diet scores ${ }^{11-13}$ or on individual food components ${ }^{14-17}$ and thus did not examine the food components of a healthy diet in relation to each other. ${ }^{11-17}$ These disparities hamper direct comparison, which is further complicated by the use of different sets of biomarkers between studies. ${ }^{17}$ Moreover, endothelial dysfunction and low-grade inflammation are closely intertwined ${ }^{18}$ and specific dietary effects might therefore be even more difficult to disentangle. ${ }^{11-14}$

In light of the above, we did a longitudinal cohort study which consisted of participants with an increased CVD risk, and we investigated, over 7 years, the associations between the consumption of fish, vegetables, fruit, alcohol-containing beverages and dairy products on the one hand and biomarkers of endothelial dysfunction [i.e. von Willebrand factor (vWf), soluble vascular cell adhesion molecule 1 (sVCAM-1), soluble endothelial selectin (sE-selectin), soluble thrombomodulin (STM), soluble intercellular adhesion molecule 1 (sICAM-1)] and lowgrade inflammation [i.e. C-reactive protein (CRP), serum amyloid A (SAA), interleukin 6 (IL-6), interleukin 8 (IL-8), tumour necrosis factor $\alpha$ (TNF- $\alpha$ ) and SICAM-1] on the other. In addition, to investigate the effects of each food group on the associations under study we examined the individual food components of each food group which have been hypothesized to have either beneficial or adverse effects on the development of CVD (i.e. fat and lean fish; raw and cooked vegetables; fresh fruits; wine and other alcohol-containing beverages; and the consumption of low-fat and high-fat dairy products). ${ }^{1-3,10}$

\section{Methods}

\section{Study population and design}

The present study used longitudinal data from the Cohort on Diabetes and Atherosclerosis Maastricht (CODAM) Study. CODAM was designed to study the effects of lifestyle, obesity, glucose and lipid metabolism, and genetics on cardiovascular complications. Briefly, 574 participants were examined at baseline (1999-2002, referred to as CODAM-1 in the current manuscript ${ }^{19-24}$ ) and were selected from a large 
population-based cohort. Inclusion criteria were Caucasian ethnicity; over 40 years of age; and at least one of the following criteria: body mass index $\geq 25 \mathrm{~kg} / \mathrm{m}^{2}$, a positive family history for type 2 diabetes, a history of gestational diabetes, the use of antihypertensive medication, and a postprandial glucose $\geq 6.0 \mathrm{mmol} / \mathrm{l}$ or glucosuria. ${ }^{19-24} \mathrm{At}$ the first follow-up examination [2006-2009, median follow-up time of 7.0 (interquartile range 6.9-7.1) years], referred to as CODAM-2, 495 participants were reexamined. The attrition rate was $14 \%$ [ $n=79$ in total; reasons for non-participation were death $(n=37)$, participation too cumbersome $(n=18)$, participant or partner suffering from disease $(n=14)$, emigration $(n=1)$, and unknown $(n=9)]$ (Figure 6.1).

Of the original 574 participants (CODAM-1), 15 were without any dietary data and two were without any biomarker data. Of the remaining 557 CODAM-1 participants, 471 had full biomarker data at both CODAM-1 and CODAM-2, and 440 had full dietary data at both CODAM-1 and CODAM-2. Of the 495 participants at CODAM-2, three were without any dietary data and two were without any biomarker data. These participants were therefore excluded. Full biomarker and dietary data at both baseline (CODAM-1) and follow-up (CODAM-2) were available in 421 participants. Missing data were not clustered in participants. Overall, 86 participants missed (individual) biomarkers at either CODAM-1 $(n=9)$ or CODAM-2 ( $n=77)$. In addition, 117 participants missed (individual) food questionnaire data at either CODAM-1 $(n=40)$ or CODAM-2 $(n=77)$ (i.e. no questionnaire or $>10 \%$ missing items within a questionnaire ${ }^{20}$ ) (Figure 6.1).

The study was approved by the Medical Ethical Committee of the Maastricht University and all participants gave their written informed consent.

\section{Dietary intake}

Dietary intake was assessed by a validated semi-quantitative Food Frequency Questionnaire (FFQ), which was originally developed for Dutch participants of the EPIC study. ${ }^{25,26}$ Based on this questionnaire the following food groups were constructed: total fish (g/wk) (with its components: fat fish, lean fish and shellfish), total vegetables (g/d) (with its components: raw vegetables, cooked vegetables and legumes), total fruit (g/d) (with its components: fresh fruit, fruit juices and apple sauce), total alcoholcontaining beverages (ml/wk) (with its components: red wine, white wine, beer and liquor) and total dairy products $(\mathrm{g} / \mathrm{d})$ [with its components: low- $(<2.0 \%)$ and high$(\geq 2.0 \%)$ fat dairy products].

Total energy $(\mathrm{kJ} / \mathrm{d})$ and alcohol $(\mathrm{g} / \mathrm{d})$ intake were calculated with the help of the extended Dutch food composition database from 2001 (NEVO). ${ }^{27}$ Alcohol consumption was analyzed as total alcohol-containing beverages (intake of alcohol per $10 \mathrm{~g} / \mathrm{d}$ ) and as a lifestyle covariate categorized into none consumers (no consumption of wine, beer or liquor), moderate consumers (men: alcohol intake $>0$ and $\leq 20 \mathrm{~g} / \mathrm{d}$; women: alcohol intake $>0$ and $\leq 10 \mathrm{~g} / \mathrm{d}$ ) and high consumers (men: alcohol intake $>20 \mathrm{~g} / \mathrm{d}$; women: alcohol intake $>10 \mathrm{~g} / \mathrm{d}$ ). 


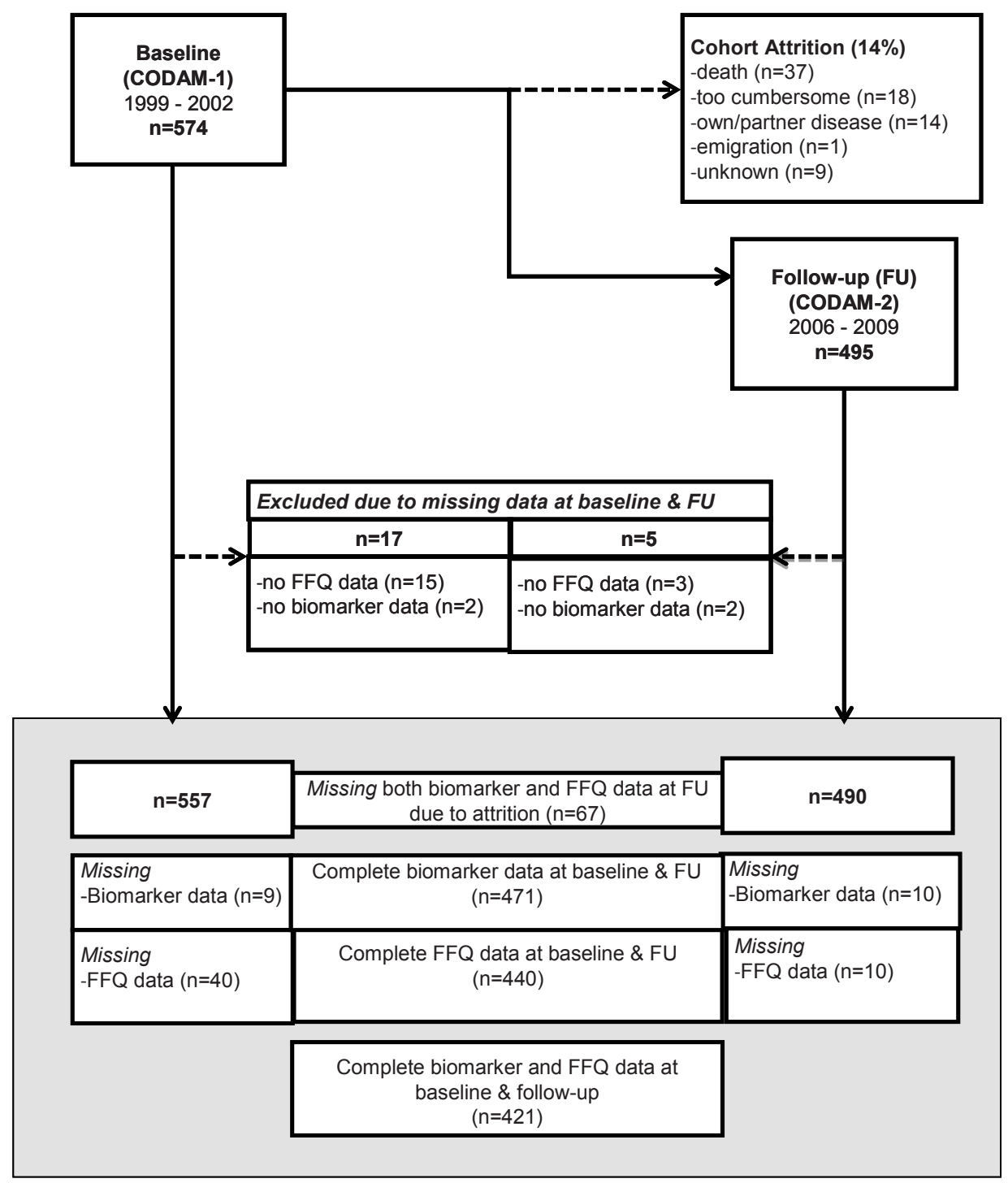

Figure 6.1 Study population and design description. Flow chart of study design and participants included in generalized estimating equations analyses.

\section{Biomarkers of endothelial dysfunction and low-grade inflammation}

Biomarkers of endothelial dysfunction (sVCAM-1, sE-selectin, STM and sICAM-1) and of low-grade inflammation (CRP, SAA, IL-6, IL-8, TNF- $\alpha$ and SICAM-1) were measured by a multi-array detection system based on electrochemiluminescence technology (MesoScaleDiscovery, Gaithersburg, MD, USA) as previously described elsewhere. ${ }^{28-30}$ 
Briefly, this system uses multi-array plates fitted with multi-electrodes per well with each electrode being coated with a different capture antibody. The assay procedure then follows that of a classic sandwich ELISA with any of the analytes of interest captured on the relevant electrode. These captured analytes were, in turn, detected by a secondary, analyte-specific, ruthenium-conjugated antibody, which is capable of emitting light after electrochemical stimulation. All plasma samples were measured on a single production lot of multi-array plates and, to further reduce inter-assay variation, both CODAM-1 and CODAM-2 samples of a participant were measured on separate designated wells on the same array plate. The intra- and inter-assay CVs were, respectively, 2.6 and $5.0 \%$ for sVCAM-1, 5.2 and $6.6 \%$ for sE-selectin, 2.2 and 6.3\% for STM, 2.5 and $4.7 \%$ for sICAM-1, 3.0 and $4.1 \%$ for CRP, 2.5 and $11.8 \%$ for SAA, 6.4 and $10.3 \%$ for IL-6, 3.8 and $5.9 \%$ for IL-8 and 4.7 and $5.8 \%$ for TNF- $\alpha$.

In addition, vWf, a biomarker of endothelial dysfunction, was measured in citrated plasma by means of ELISA. vWf had already been measured in CODAM-1. ${ }^{22}$ For the present study, the ELISA was also assayed in citrated plasma samples of CODAM- 2 . Briefly, vWf rabbit antibody was used as a catching antibody and a peroxidaseconjugate rabbit antibody as detecting antibody. ${ }^{31}$ Levels were expressed as a percentage of $\mathrm{vWf}$ detected in pooled citrated plasma of healthy volunteers. Intraand inter-assay CVs were 3.4 and $6.0 \%$ in CODAM-1 and 3.3 and $7.6 \%$ in CODAM-2.

Some of the biomarkers had already been determined in CODAM-1 by conventional single-biomarker laboratory techniques (hs-CRP by immunoturbidimetry and sVCAM-1, sE-selectin, sICAM-1, SAA and IL- 6 by ELISA ${ }^{21,22}$ ). For these biomarkers, we realigned the absolute levels as obtained by these conventional techniques to those as obtained by the multi-array technique ${ }^{30}$ and used the average of both methods, after realignment, in the analyses, as repeated measurements of a given biologic phenomenon offer more mathematical precision than a single measurement. ${ }^{32}$

\section{Other measurements}

Medication use, physical activity and smoking habits were assessed by the same questionnaire in CODAM-1 and CODAM-2. Height, weight, blood pressure, body mass index and glucose metabolism status were determined in CODAM-1 and CODAM-2 as previously described. ${ }^{19-24}$ Self-reported smoking behaviour was classified as neversmokers, ex-smokers and current smokers. ${ }^{24}$ The prevalence of CVD (at CODAM-1 only) was based on self-reports of myocardial infarction, coronary bypass surgery, stent placement or balloon dilatation, transient ischaemic attack or stroke, signs of myocardial infarction or ischaemia on a 12-lead electrocardiogram (Minnesota codes 1.1 to $1.3,4.1$ to $4.3,5.1$ to 5.3 or 7.1 ) and/or self-reported non-traumatic limb amputation or a measured ankle-arm index $<0.9 .^{21,22}$ 


\section{Statistical analyses}

All analyses were performed with SPSS (Statistical Package for Social Sciences, version 20 , IBM Corp., USA). Data are presented as mean ( $\pm S D$ ), median (interquartile range) or proportions. Variables with a skewed distribution were natural log-transformed where appropriate (CRP, SAA, IL-6, IL-8, TNF- $\alpha$ and triglycerides). Independent T-test, Mann-Whitney $U$ test (for skewed data) or chi-square test (for proportions) was used to investigate whether individuals without follow-up biomarker data $(n=94)$ differed, at baseline (i.e. CODAM-1), from those with follow-up biomarker data $(n=480)$ (Figure 6.1).

For reasons of statistical efficiency and to reduce the influence of biological variability $^{28,29,33}$ overall biomarker scores were calculated for endothelial dysfunction and low-grade inflammation as follows: for each individual biomarker, a Z-score (based on normally distributed or log-transformed biomarker data as indicated) was calculated according to the formula: (individual value - population mean) / population SD (with the population mean and SD for each individual biomarker based on its average of CODAM-1 and CODAM-2). The resulting individual biomarker Z-scores were then averaged into the overall biomarker scores for both endothelial dysfunction and low-grade inflammation. The endothelial dysfunction score consisted of the biomarkers sVCAM-1, SE-selectin, STM, SICAM-1 and VWf and the low-grade inflammation score consisted of the biomarkers CRP, SAA, IL-6, IL-8, TNF- $\alpha$ and sICAM-1 (both monocytes and the endothelium express SICAM-1; therefore, sICAM-1 was included in both overall scores ${ }^{34}$ ).

Generalized estimating equations (GEE) were used to examine the longitudinal associations between food consumption and the endothelial dysfunction and the lowgrade inflammation scores. The advantage of GEE is that GEE uses a pre-specified correlation structure that takes into account any presumed correlation between repeated observations over time within the same subjects. In addition, GEE is capable of handling data with a varying number of observations between participants. The latter minimizes any effect of bias due to missing data. ${ }^{35}$

We examined the consumption of (a) total fish (fat fish and lean fish), (b) total vegetables (raw and cooked vegetables), (c) total fruit (fresh fruit and fruit juice), (d) total alcohol-containing beverages (wine, beer and liquor) and (e) total dairy products (low-fat and high-fat dairy products) as determinants of the overall endothelial dysfunction and overall low-grade inflammation scores. The models were adjusted for sex, age, glucose metabolism status and energy intake (model 1) and then additionally adjusted for body mass index, physical activity, alcohol categories and smoking status (model 2).

To investigate whether the longitudinal associations (if any) were primarily determined by the between- or the within-subjects effects over time, we additionally investigated, with the use of linear regression analyses, the extent to which any changes in dietary variables within subjects were associated with any changes in biomarkers of endothelial dysfunction or low-grade inflammation within subjects 
(please see Appendix 6 for details). Finally, as the presence of clinical disease has been suggested to modify effects of diet on CVD ${ }^{10,36}$, we used interaction terms between clinical disease at CODAM-1 and food consumption to examine any such effects. In the present study, clinical disease was defined as the presence of either CVD $(n=102)$, diabetes type $2(n=87)$, or both $(n=50)$.

A two-sided $p$-value $<0.05$ and for interaction $<0.10$ was considered statistically significant.

\section{Results}

Table 6.1 shows the characteristics of the 557 participants at baseline (CODAM-1). Individuals who did not contribute data at follow-up (CODAM-2) were at baseline (CODAM-1) older, had a significantly higher prevalence of diabetes, a higher systolic blood pressure, a lower energy intake, a lower liquor (but similar wine and beer) consumption and higher levels of SVCAM-1, SICAM-1, CRP, IL-6 and IL-8 (data not shown).

Table 6.1 Characteristics of the study population, diet and biomarkers at baseline and at follow-up.

\begin{tabular}{lcc}
\hline & \multicolumn{2}{c}{ All ever measured $\mathrm{n}=557$} \\
\cline { 2 - 3 } & $\begin{array}{c}\text { Baseline } \\
(\mathrm{n}=548)\end{array}$ & $\begin{array}{c}\text { Follow-up } \\
(\mathrm{n}=480)\end{array}$ \\
\hline Age, years & $59.6 \pm 6.9$ & $66.2 \pm 7.0$ \\
Women, $\mathrm{n}$ (\%) & $211(38.5 \%)$ & $185(38.5 \%)$ \\
Glucose metabolism status * & & \\
$\quad$ Normal glucose metabolism, \% & 52.4 & 24.4 \\
Impaired glucose metabolism, \% & 22.6 & 34.4 \\
$\quad$ Diabetes mellitus type 2, \% & 25.0 & $28.5 \pm 4.2$ \\
Body mass index, kg/m ${ }^{2}$ & $28.5 \pm 4.2$ & $7.0 \pm 4.6$ \\
Physical activity, x 1000 METs/wk & $6.6 \pm 4.2$ & $13.6 / 55.5 / 30.9$ \\
Alcohol categories (none/moderate/high), \% & $13.0 / 53.1 / 33.9$ & $24.7 / 59.9 / 15.2$ \\
Smoking status (never, ex, current) \% & $29.0 / 50.0 / 21.0$ & $147 \pm 22$ \\
Systolic blood pressure, mmHg & $140 \pm 19$ & $83 \pm 10$ \\
Diastolic blood pressure, mmHg & $82 \pm 9$ & 59.1 \\
Anti-hypertensive medication use, \% & 38.3 & 44.1 \\
Lipid-lowering medication, \% & 19.2 & $(\mathrm{n} . \mathrm{a})$. \\
Prior cardiovascular disease, \% & 27.4 & \\
\hline Diet & & $8879 \pm 2576$ \\
\hline Energy intake, kJ/d & $9341 \pm 2696$ & $16(6-33)$ \\
Total fish, g/wk & $76(25-117)$ & $98(31-128)$ \\
Fat fish, g/wk & $10(4-25)$ & $57(16-97)$ \\
\hline Lean fish, g/wk & $45(14-82)$ & \\
\hline
\end{tabular}




\begin{tabular}{|c|c|c|}
\hline & \multicolumn{2}{|c|}{ All ever measured $n=557$} \\
\hline & $\begin{array}{l}\text { Baseline } \\
(n=548)\end{array}$ & $\begin{array}{l}\text { Follow-up } \\
(n=480)\end{array}$ \\
\hline Total vegetables, $\mathrm{g} / \mathrm{d}$ & $139 \pm 59$ & $135 \pm 60$ \\
\hline Raw vegetables, $\mathrm{g} / \mathrm{d}$ & $32(16-52)$ & $26(13-50)$ \\
\hline Cooked vegetables, $\mathrm{g} / \mathrm{d}$ & $93 \pm 45$ & $91 \pm 48$ \\
\hline Total fruit, $\mathrm{g} / \mathrm{d}$ & $262 \pm 154$ & $271 \pm 167$ \\
\hline Fresh fruit, $\mathrm{g} / \mathrm{d}$ & $162 \pm 118$ & $180 \pm 129$ \\
\hline Fruit juice, $\mathrm{ml} / \mathrm{d}$ & $57(19-133)$ & $39(11-124)$ \\
\hline Total alcohol-containing beverages, (i.e. alcohol) g/d & $8.5(1.3-21.4)$ & $7.9(1.3-19.1)$ \\
\hline Wine, $\mathrm{ml} / \mathrm{wk}$ & $93(0-493)$ & $100(0-600)$ \\
\hline Beer, $\mathrm{ml} / \mathrm{wk}$ & $93(0-1000)$ & $47(0-800)$ \\
\hline Liquor, $\mathrm{ml} / \mathrm{wk}$ & $3(0-77)$ & $0(0-50)$ \\
\hline Total dairy products, g/d & $252(126-407)$ & $237(129-404)$ \\
\hline Low-fat dairy products (<2.0\% fat), g/d & $93(21-216)$ & $91(21-219)$ \\
\hline High-fat dairy products ( $\geq 2.0 \%$ fat), $\mathrm{g} / \mathrm{d}$ & $135(84-193)$ & $127(79-187)$ \\
\hline \multicolumn{3}{|l|}{ Endothelial dysfunction } \\
\hline Endothelial dysfunction score, SD & $-0.060 \pm 0.573$ & $0.068 \pm 0.665$ \\
\hline von Willebrand factor, $\%$ & $132 \pm 62$ & $148 \pm 52$ \\
\hline Soluble endothelial selectin, $\mu \mathrm{g} / \mathrm{l}$ & $8.7 \pm 3.7$ & $7.6 \pm 3.6$ \\
\hline Soluble thrombomodulin, $\mu \mathrm{g} / \mathrm{l}$ & $2.75 \pm 0.65$ & $2.95 \pm 0.76$ \\
\hline Soluble vascular cellular adhesion molecule $1, \mu \mathrm{g} / \mathrm{l}$ & $340 \pm 70$ & $365 \pm 87$ \\
\hline Soluble intercellular adhesion molecule $1, \mu \mathrm{g} / \mathrm{l}$ & $221 \pm 49$ & $224 \pm 55$ \\
\hline \multicolumn{3}{|l|}{ Low-grade inflammation } \\
\hline Low-grade inflammation score, SD & $-0.061 \pm 0.580$ & $0.070 \pm 0.660$ \\
\hline C-reactive protein, $\mathrm{mg} / \mathrm{l}$ & $2.0(0.9-3.9)$ & $2.0(0.9-3.9)$ \\
\hline Serum amyloid $A, \mathrm{mg} / \mathrm{l}$ & $1.4(1.0-2.3)$ & $1.4(0.9-2.8)$ \\
\hline Interleukin-6, ng/l & $1.5(1.1-2.2)$ & $1.5(1.1-2.5)$ \\
\hline Interleukin-8, ng/l & $4.4(3.6-5.5)$ & $5.2(4.3-6.7)$ \\
\hline Tumour necrosis factor $\alpha, \mathrm{ng} / \mathrm{l}$ & $6.2(5.3-7.5)$ & $6.6(5.6-8.3)$ \\
\hline Soluble intercellular adhesion molecule $1, \mu \mathrm{g} / \mathrm{l}$ & $221 \pm 49$ & $224 \pm 55$ \\
\hline
\end{tabular}

Data are reported as mean $\pm \mathrm{SD}$, median (interquartile range), or percentage, as appropriate. ${ }^{*}$ normal glucose metabolism: fasting plasma glucose $<6.1 \mathrm{mmol} / \mathrm{l}$ and 2-hour post-load plasma glucose $<7.8 \mathrm{mmol} / \mathrm{l}$; impaired glucose metabolism: impaired fasting plasma glucose between $6.1 \mathrm{mmol} / \mathrm{l}$ and $7.0 \mathrm{mmol} / \mathrm{l}$ and impaired glucose tolerance defined as a 2-hour post-load plasma glucose between 7.8 and $11.1 \mathrm{mmol} / \mathrm{l}$; diabetes mellitus type 2: fasting plasma glucose $\geq 7.0 \mathrm{mmol} / \mathrm{l}$ and/or 2-hour post-load plasma glucose $\geq 11.1 \mathrm{mmol} / \mathrm{l}$; METs, metabolic equivalents score (including average weekly time spent on different activities multiplied by their intensity); alcohol categories: none, moderate (0-10 g/d for women; 0-20 g/d for men), high (>10 g/d for women; $>20 \mathrm{~g} / \mathrm{d}$ for men).

\section{Associations between food consumption and endothelial dysfunction}

Fish: After adjustment for sex, age, glucose metabolism status and energy intake (model 1), and other potential confounders (model 2), total fish consumption (per $100 \mathrm{~g} / \mathrm{wk}$ ) was inversely associated with the overall endothelial dysfunction score (higher score indicates worse function): $\beta$ (95\% Cl) $-0.027(-0.051 ;-0.004)$ (Table 6.2, model 2). Further food component analyses showed an inverse association for lean fish consumption: $-0.038(-0.072 ;-0.005)$. 
Table 6.2 Longitudinal associations between food consumption and endothelial dysfunction and lowgrade inflammation during a 7-year follow-up

\begin{tabular}{|c|c|c|c|c|c|}
\hline & \multirow[b]{2}{*}{ Model } & \multicolumn{2}{|c|}{$\begin{array}{c}\text { Endothelial dysfunction } \\
\text { score }\end{array}$} & \multicolumn{2}{|c|}{$\begin{array}{c}\text { Low-grade inflammation } \\
\text { score }\end{array}$} \\
\hline & & $\mathrm{B}$ & $95 \% \mathrm{Cl}$ & $\beta$ & $95 \% \mathrm{Cl}$ \\
\hline \multirow[t]{2}{*}{ Total fish, $100 \mathrm{~g} / \mathrm{wk}$} & 1 & -0.023 & $-0.044 ;-0.003 *$ & -0.010 & $-0.037 ; 0.017$ \\
\hline & 2 & -0.027 & $-0.051 ;-0.004 *$ & -0.012 & $-0.041 ; 0.016$ \\
\hline \multirow[t]{2}{*}{ Fat fish, $100 \mathrm{~g} / \mathrm{wk}$} & 1 & -0.019 & $-0.079 ; 0.041$ & 0.002 & $-0.077 ; 0.080$ \\
\hline & 2 & -0.032 & $-0.108 ; 0.045$ & -0.016 & $-0.105 ; 0.073$ \\
\hline \multirow[t]{2}{*}{ Lean fish, $100 \mathrm{~g} / \mathrm{wk}$} & 1 & -0.035 & $-0.065 ;-0.005 *$ & -0.018 & $-0.056 ; 0.021$ \\
\hline & 2 & -0.038 & $-0.072 ;-0.005 *$ & -0.018 & $-0.058 ; 0.021$ \\
\hline \multirow[t]{2}{*}{ Total vegetables, $100 \mathrm{~g} / \mathrm{d}$} & 1 & -0.037 & $-0.086 ; 0.012$ & 0.004 & $-0.053 ; 0.061$ \\
\hline & 2 & -0.034 & $-0.080 ; 0.013$ & 0.011 & $-0.044 ; 0.067$ \\
\hline \multirow{2}{*}{ Raw vegetables, $100 \mathrm{~g} / \mathrm{d}$} & 1 & -0.097 & $-0.191 ;-0.003 *$ & -0.039 & $-0.152 ; 0.074$ \\
\hline & 2 & -0.095 & $-0.191 ; 0.000 *$ & -0.031 & $-0.149 ; 0.086$ \\
\hline \multirow[t]{2}{*}{ Cooked vegetables, $100 \mathrm{~g} / \mathrm{d}$} & 1 & -0.030 & $-0.097 ; 0.037$ & 0.001 & $-0.075 ; 0.078$ \\
\hline & 2 & -0.025 & $-0.089 ; 0.040$ & 0.013 & $-0.060 ; 0.086$ \\
\hline \multirow[t]{2}{*}{ Total fruit, $100 \mathrm{~g} / \mathrm{d}$} & 1 & 0.008 & $-0.010 ; 0.027$ & -0.017 & $-0.040 ; 0.006$ \\
\hline & 2 & 0.010 & $-0.009 ; 0.028$ & -0.012 & $-0.035 ; 0.010$ \\
\hline \multirow[t]{2}{*}{ Fresh fruit, $100 \mathrm{~g} / \mathrm{d}$} & 1 & -0.007 & $-0.031 ; 0.017$ & -0.031 & $-0.060 ;-0.002 *$ \\
\hline & 2 & -0.002 & $-0.026 ; 0.021$ & -0.022 & $-0.051 ; 0.007$ \\
\hline \multirow[t]{2}{*}{ Fruit juice, $100 \mathrm{ml} / \mathrm{d}$} & 1 & 0.030 & $-0.002 ; 0.062$ & -0.006 & $-0.042 ; 0.030$ \\
\hline & 2 & 0.027 & $-0.005 ; 0.059$ & -0.007 & $-0.043 ; 0.029$ \\
\hline \multirow{2}{*}{$\begin{array}{l}\text { Total alcohol-containing } \\
\text { beverages, } 10 \mathrm{~g} / \mathrm{d}\end{array}$} & 1 & -0.011 & $-0.034 ; 0.012$ & -0.003 & $-0.027 ; 0.021$ \\
\hline & 2 & -0.003 & $-0.038 ; 0.033$ & 0.005 & $-0.027 ; 0.037$ \\
\hline \multirow[t]{2}{*}{ Wine, $100 \mathrm{ml} / \mathrm{wk}$} & 1 & -0.005 & $-0.010 ;-0.001 *$ & -0.007 & $-0.012 ;-0.001 *$ \\
\hline & 2 & -0.004 & $-0.010 ; 0.001$ & -0.006 & $-0.013 ; 0.001$ \\
\hline \multirow[t]{2}{*}{ Beer, $100 \mathrm{ml} / \mathrm{wk}$} & 1 & -0.001 & $-0.003 ; 0.001$ & 0.000 & $-0.002 ; 0.002$ \\
\hline & 2 & -0.001 & $-0.003 ; 0.002$ & 0.000 & $-0.002 ; 0.002$ \\
\hline \multirow[t]{2}{*}{ Liquor, 100 ml/wk } & 1 & 0.011 & $-0.013 ; 0.034$ & 0.020 & $-0.002 ; 0.042$ \\
\hline & 2 & 0.014 & $-0.011 ; 0.039$ & 0.021 & $-0.002 ; 0.044$ \\
\hline \multirow[t]{2}{*}{ Total dairy products, $100 \mathrm{~g} / \mathrm{d}$} & 1 & 0.006 & $-0.012 ; 0.025$ & 0.004 & $-0.016 ; 0.024$ \\
\hline & 2 & 0.009 & $-0.010 ; 0.027$ & 0.009 & $-0.011 ; 0.028$ \\
\hline \multirow[t]{2}{*}{ Low-fat dairy products, $100 \mathrm{~g} / \mathrm{d}$} & 1 & -0.007 & $-0.027 ; 0.012$ & -0.003 & $-0.026 ; 0.021$ \\
\hline & 2 & -0.005 & $-0.025 ; 0.014$ & 0.003 & $-0.021 ; 0.027$ \\
\hline \multirow[t]{2}{*}{ High-fat dairy products, $100 \mathrm{~g} / \mathrm{d}$} & 1 & 0.065 & $0.006 ; 0.124 *$ & 0.032 & $-0.025 ; 0.089$ \\
\hline & 2 & 0.070 & $0.009 ; 0.131 *$ & 0.040 & $-0.015 ; 0.095$ \\
\hline
\end{tabular}

$\beta$, longitudinal regression coefficients as obtained from generalized estimating equations indicate the longitudinal relationship between fish, vegetable, fruit, alcohol-containing beverage and dairy product consumption on the one hand, and overall endothelial dysfunction and low-grade inflammation scores on the other; $95 \% \mathrm{Cl}, 95 \%$ confidence interval; * $p$-value $<0.05 ; n=557$. Food group components, such as fat and lean fish, were analyzed in separate models. Model 1: adjusted for sex, age, glucose metabolism status and energy intake. Model 2: model $1+$ adjusted for body mass index, physical activity, alcohol categories and current smoking.

Vegetables: Total vegetable consumption (per $100 \mathrm{~g} / \mathrm{d}$ ) was not significantly associated with the overall endothelial dysfunction score. Further food component analyses showed that, after adjustments for sex, age, glucose metabolism status and energy intake (model 1), and other potential confounders (model 2), raw vegetable 
consumption was inversely associated with the overall endothelial dysfunction score: $-0.095(-0.191 ; 0.000)$.

Fruit: total fruit consumption (per $100 \mathrm{~g} / \mathrm{d}$ ) was not significantly associated with the overall endothelial dysfunction score. Further food component analyses showed no significant association for fresh fruit and fruit juice consumption.

Alcohol-containing beverages: total alcohol-containing beverage consumption (per $10 \mathrm{~g} / \mathrm{d}$ ) was not significantly associated with the overall endothelial dysfunction score. Further food component analyses showed, after adjustments for sex, age, glucose metabolism status and energy intake (model 1 ), and other potential confounders (model 2), no significant associations for wine, beer and liquor consumption.

Dairy products: total dairy product consumption (per $100 \mathrm{~g} / \mathrm{d}$ ) was not significantly associated with the overall endothelial dysfunction score. Further food component analyses showed, after adjustments for sex, age, glucose metabolism status and energy intake (model 1), and other potential confounders (model 2), that high-fat dairy product consumption was positively associated with the overall endothelial dysfunction score: $0.070(0.009 ; 0.131)$.

\section{Associations between food consumption and low-grade inflammation}

Fish, vegetable, fruit, alcohol-containing beverages and dairy product consumption were not significantly associated with the overall low-grade inflammation score. However, food component analyses showed that, after adjustments for sex, age, glucose metabolism status and energy intake (model 1), and other potential confounders (model 2), wine consumption was inversely associated with the overall low-grade inflammation score: $-0.006 \quad(-0.013 ; 0.001)$, although this was not statistically significant $(p=0.092)$. In contrast, liquor consumption was positively associated with the overall low-grade inflammation score: 0.021 (-0.002;0.044), although this was not statistically significant $(p=0.077)$.

\section{Analyses of changes}

Overall, within-subject changes in dietary variables were not associated with withinsubject changes in biomarkers of endothelial dysfunction or low-grade inflammation over time (please see Appendix 6 for details). The reported longitudinal associations (by GEE) were therefore primarily determined by the between-subject associations over the 7-year study period.

\section{Results according to the presence or absence of clinical disease at baseline}

The association between diet and endothelial dysfunction and low-grade inflammation may differ according to the presence of clinical disease (i.e. CVD and/or diabetes). ${ }^{10,36}$ In particular, it has been suggested that the consumption of dairy 
products may be unfavourable in $C V D^{37}$, whereas the consumption of fresh fruit may be beneficial. ${ }^{1,36,38}$ If we tested these hypotheses the results showed that the consumption of total- and high-fat dairy products were positively associated with the overall low-grade inflammation score in participants with clinical disease (Table 6.3; $p_{\text {interaction }}$ : 0.019 and 0.003 , respectively). In addition, the results for fresh fruit showed that the consumption of fresh fruit was inversely associated with the overall lowgrade inflammation score in participants with clinical disease (Table 6.3; $p_{\text {interaction: }}$ : 0.051).

Table 6.3 Longitudinal associations between food consumption and low-grade inflammation during a 7year follow-up, stratified according to the presence of clinical disease at baseline.

\begin{tabular}{|c|c|c|c|c|}
\hline & \multicolumn{4}{|c|}{ Low-grade inflammation } \\
\hline & \multicolumn{2}{|c|}{ free of clinical disease } & \multicolumn{2}{|c|}{ presence of clinical disease } \\
\hline & $\beta$ & $95 \% \mathrm{Cl}$ & $\beta$ & $95 \% \mathrm{Cl}$ \\
\hline Fresh fruit, $100 \mathrm{~g} / \mathrm{d}$ & -0.005 & $-0.038 ; 0.027$ & -0.074 & $-0.133 ;-0.015^{*}$ \\
\hline Total dairy products, $100 \mathrm{~g} / \mathrm{d}$ & -0.002 & $-0.026 ; 0.022$ & 0.034 & $0.002 ; 0.066 *$ \\
\hline High-fat dairy products, $100 \mathrm{~g} / \mathrm{d}$ & -0.018 & $-0.074 ; 0.038$ & 0.100 & $0.019 ; 0.182 *$ \\
\hline
\end{tabular}

$\beta$, longitudinal regression coefficients as obtained from generalized estimating equations indicate the longitudinal relationship between fresh fruit and total and high-fat dairy product consumption on the one hand, and the overall low-grade inflammation score, on the other, according to strata of clinical disease; 95\% Cl, 95\% confidence interval; ${ }^{*} \mathrm{p}$-value $<0.05 ; \mathrm{n}=239$ participants with clinical disease: either CVD $(n=102)$, diabetes type $2(n=87)$, or both $(n=50) ; n=318$ participants free of clinical disease; models adjusted for sex, age, glucose metabolism status, energy intake, body mass index, physical activity, alcohol categories and current smoking.

\section{Additional analyses}

When we repeated the GEE analyses on complete cases $(n=421)$, the results did not materially change (data not shown). Additional adjustment for each of the other food groups (i.e. total fish, total vegetables, total fruit, total alcohol-containing beverages and total dairy products) or for lipid-lowering and anti-hypertensive medication and prior CVD did not materially change the results (data not shown). Further adjustment of the association between wine consumption and the overall low-grade inflammation score for total alcohol (i.e. ethanol) intake showed the following result: $\beta(95 \% \mathrm{Cl})$ $-0.007(-0.015 ; 0.000), p=0.052$. To investigate whether the association for high-fat dairy product consumption was primarily determined by (high-fat) cheese consumption $^{27}$, data were re-analyzed without the high-fat cheese component. This did not materially change the results (data not shown). Alternative analyses for individual biomarkers gave results similar to those presented here (Figure 6.2). 


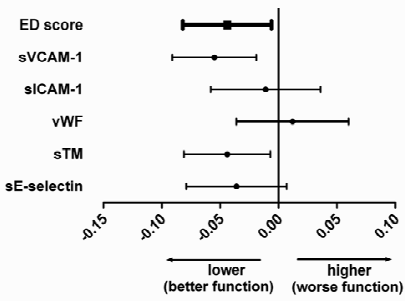

Vegetables (raw) $100 \mathrm{~g} / \mathrm{d}$

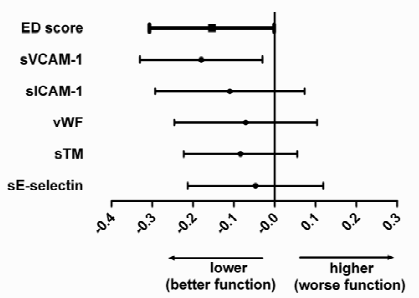

Wine $100 \mathrm{ml} / \mathrm{wk}$

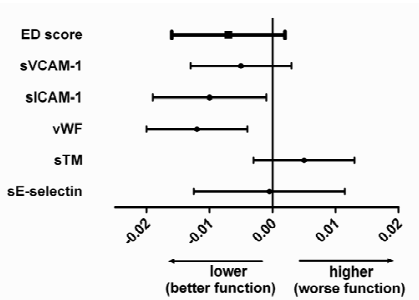

High-fat dairy products $100 \mathrm{~g} / \mathrm{d}$

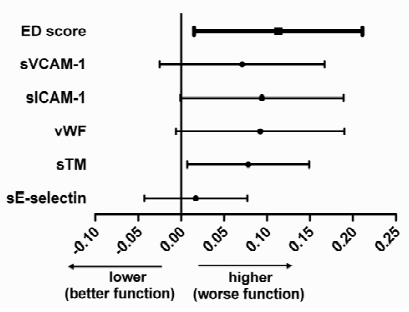

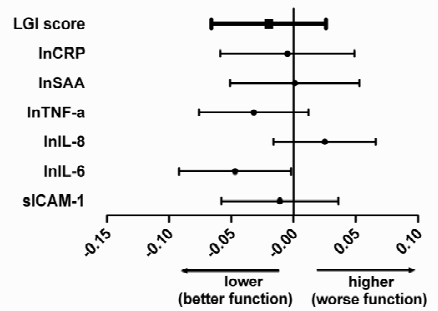
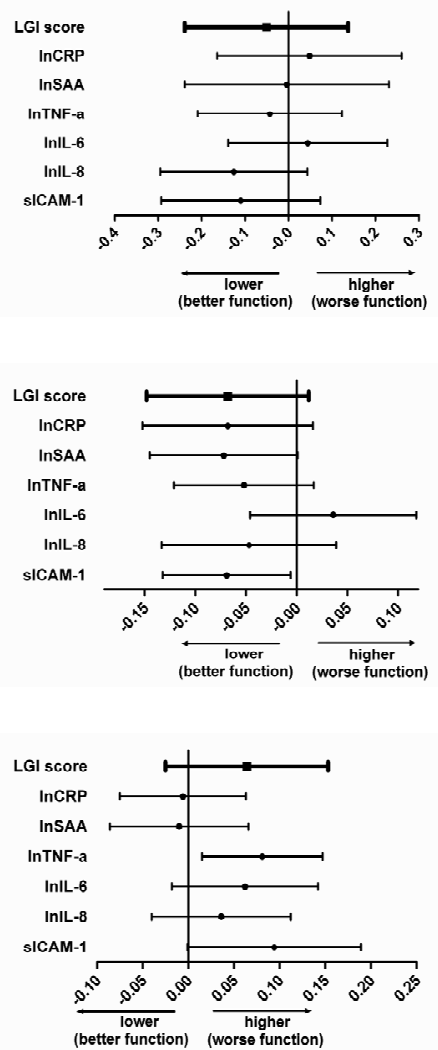

Figure 6.2 Associations between diet and biomarkers. Left panel: biomarkers of endothelial dysfunction; Right panel: biomarkers of low-grade inflammation. Data are standardized longitudinal regression coefficients as obtained from GEE, regarding the associations between (A) total fish consumption, (B) vegetable (raw) consumption, (C) wine consumption, and (D) high-fat dairy product consumption and the overall endothelial dysfunction and low-grade inflammation scores and each of the individual biomarkers. Whiskers indicate the $95 \%$ confidence intervals; $n=557$. To facilitate the comparison of results between individual biomarkers and the overall biomarker scores within a panel, each outcome variable, which is expressed in a different scale unit, was standardized to a comparable unit by calculation of a Z-score: (the individuals' value - the population mean) / the population SD. Results are adjusted for sex, age, energy intake, glucose metabolism status, body mass index, physical activity, alcohol categories and current smoking. 


\section{Discussion}

The present study is the first longitudinal investigation on the association between habitual diet and an extensive set of biomarkers of endothelial dysfunction and lowgrade inflammation. The study had three main findings. First, a higher consumption of fish was associated with less endothelial dysfunction, whereas the consumption of dairy products, fruit, vegetables and alcohol-containing beverages showed no association with endothelial dysfunction. However, analyses at the food component level showed that a higher consumption of lean fish, raw vegetables, and a lower consumption of high-fat dairy products and fruit juice was associated with less endothelial dysfunction, although the result for fruit juice was borderline statistically significant $(p<0.10)$. Second, the consumption of fish, vegetables, fruit, alcoholcontaining beverages and dairy products was not associated with low-grade inflammation. However, analyses at the food component level showed that a higher consumption of, in particular, wine and a lower consumption of liquor were, albeit borderline statistically significantly (both $\mathrm{p}<0.10$ ), associated with less low-grade inflammation. Third, the results showed that the consumption of fresh fruit impacted favourably, whereas the consumption of total and high-fat dairy products impacted unfavourably on low-grade inflammation in those with diabetes and/or CVD. The present results thereby provide evidence that a diet rich in fish, raw vegetables and fresh fruit, with wine and less high-fat dairy products may favourably impact upon (the development of) $C V D^{5-9}$ and that dietary recommendations may have to be intensified, particularly, in patients with diabetes and/or CVD. ${ }^{10}$

The question remains nevertheless how a healthy diet may biochemically modify endothelial dysfunction and low-grade inflammation. Additional adjustments in the present study suggest independent roles for fish, raw vegetables, fresh fruit, wine and high-fat dairy products. With regard to fish and plant-derived foods, (micro-) nutrients such as long-chain polyunsaturated fat and fibre, anti-oxidant vitamins and phytochemicals, respectively, may promote endothelial cell membrane stability, inhibit intracellular inflammatory signalling pathways and reduce oxidative stress. ${ }^{36,39-42}$ Similar mechanisms have been suggested to play a role with regard to the consumption of, particularly, polyphenols and flavonoids in red wine. . $^{17,41,43}$ Alternatively, it has been suggested that diet may cause a reduction in arterial stiffness ${ }^{38,44}$ by shifting the balance between endothelium-derived vasodilatory and vasoconstrictory substances towards vasodilation ${ }^{28,29}$, which may impact directly upon arterial stiffness-related CVD such as myocardial infarction and stroke. ${ }^{45,46}$

Interestingly, our results showed that the analyses on the association between red wine and endothelial dysfunction remained largely unchanged if these analyses were additionally adjusted for total alcohol (i.e. ethanol) intake. The latter finding thereby supports the idea that micronutrients in red wine itself, other than the alcohol component, favourably impact upon endothelial dysfunction. ${ }^{41,43}$ 
With regard to dairy product consumption the present study showed that high-fat dairy product consumption was positively associated with endothelial dysfunction and, in participants with diabetes and/or CVD, with low-grade inflammation. Thereby, the present study supports a recent observation which showed that high-fat, but not low-fat, dairy product consumption was associated with CVD mortality ${ }^{37}$ and provides an underlying pathway how dairy product consumption is associated with CVD.

The present data expand previous knowledge obtained from cross-sectional studies $^{11-17}$ and support the hypothesis that a habitual healthy diet over a long period of time is associated with less endothelial dysfunction and less low-grade inflammation. Additional analyses showed that the associations were primarily driven by differences between-subjects as opposed to changes within-subjects (please see Appendix 6 for details). The within-subject analyses are however more susceptible to measurement (and thus type 1) error as it has been shown that diet remains fairly stable over a long period of time ${ }^{47}$ and any changes in diet are relatively small.

The present study has some limitations. First, the endothelium has a heterogeneous set of functions ${ }^{48-50}$, which can be represented by an array of biomarkers ${ }^{50}$ including flow-mediated vasodilation, a functional measure of endothelial function. Flowmediated vasodilation was not available in this cohort but this measure could have provided additional insight into the complex relationship between diet and endothelial function. ${ }^{39-41,48-50}$ Second, a healthy survivor effect may have caused us to underestimate the associations between diet and endothelial dysfunction and/or lowgrade inflammation. We have in part circumvented this problem by analyzing data from all participants ever measured instead of confining the analyses to those with complete data at baseline and follow-up. Associations may have also been underestimated by the fact that it is known that "unhealthy" individuals may underreport unhealthy dietary habits. ${ }^{51}$ Third, we cannot fully exclude residual confounding, despite the fact that the results remained unchanged after additional adjustment for other food groups (i.e. total fish, total vegetables, total fruit, total alcohol-containing beverages and total dairy products), lipid-lowering and antihypertensive medication and prior CVD. Finally, intrinsic to an overall score is the assumption that each biomarker in the score carries similar weight. It is however unknown whether this is the case for each individual biomarker in the overall score with regard to CVD risk. The chosen approach may therefore not optimally reflect pathophysiology.

In conclusion, this 7-year longitudinal cohort study showed that a habitual healthy diet rich in fish, raw vegetables and fresh fruit, with wine and low in high-fat dairy products may modify processes that are important in atherothrombosis and thereby favourably influence the development of CVD. 


\section{References}

1. Knoops KT, de Groot LC, Kromhout D, Perrin AE, Moreiras-Varela O, Menotti A, van Staveren WA. Mediterranean diet, lifestyle factors, and 10-year mortality in elderly European men and women: the HALE project. Jama 2004;292:1433-1439.

2. Mitrou PN, Kipnis V, Thiebaut AC, Reedy J, Subar AF, Wirfalt E, Flood A, Mouw T, Hollenbeck AR, Leitzmann MF, Schatzkin A. Mediterranean dietary pattern and prediction of all-cause mortality in a US population: results from the NIH-AARP Diet and Health Study. Arch Intern Med 2007;167:24612468.

3. Hu FB, Rimm EB, Stampfer MJ, Ascherio A, Spiegelman D, Willett WC. Prospective study of major dietary patterns and risk of coronary heart disease in men. Am J Clin Nutr 2000;72:912-921.

4. Hansson GK. Inflammation, atherosclerosis, and coronary artery disease. N Engl J Med 2005;352:1685-1695.

5. Blankenberg S, Rupprecht HJ, Bickel C, Peetz D, Hafner G, Tiret L, Meyer J. Circulating cell adhesion molecules and death in patients with coronary artery disease. Circulation 2001;104:1336-1342.

6. de Jager J, Dekker JM, Kooy A, Kostense PJ, Nijpels G, Heine RJ, Bouter LM, Stehouwer CD. Endothelial dysfunction and low-grade inflammation explain much of the excess cardiovascular mortality in individuals with type 2 diabetes: the Hoorn Study. Arterioscler Thromb Vasc Biol 2006;26:1086-1093.

7. Jager A, van Hinsbergh VW, Kostense PJ, Emeis JJ, Yudkin JS, Nijpels G, Dekker JM, Heine RJ, Bouter LM, Stehouwer CD. von Willebrand factor, C-reactive protein, and 5-year mortality in diabetic and nondiabetic subjects: the Hoorn Study. Arterioscler Thromb Vasc Biol 1999;19:3071-3078.

8. Morange PE, Simon C, Alessi MC, Luc G, Arveiler D, Ferrieres J, Amouyel P, Evans A, Ducimetiere P, Juhan-Vague I. Endothelial cell markers and the risk of coronary heart disease: the Prospective Epidemiological Study of Myocardial Infarction (PRIME) study. Circulation 2004;109:1343-1348.

9. Ridker PM, Hennekens $\mathrm{CH}$, Buring JE, Rifai N. C-reactive protein and other markers of inflammation in the prediction of cardiovascular disease in women. N Engl J Med 2000;342:836-843.

10. Lichtenstein AH, Appel $\amalg$, Brands M, Carnethon M, Daniels S, Franch HA, Franklin B, Kris-Etherton P, Harris WS, Howard B, Karanja N, Lefevre M, Rudel L, Sacks F, Van Horn L, Winston M, Wylie-Rosett J. Diet and lifestyle recommendations revision 2006: a scientific statement from the American Heart Association Nutrition Committee. Circulation 2006;114:82-96.

11. Nettleton JA, Steffen LM, Mayer-Davis EJ, Jenny NS, Jiang R, Herrington DM, Jacobs DR, Jr. Dietary patterns are associated with biochemical markers of inflammation and endothelial activation in the Multi-Ethnic Study of Atherosclerosis (MESA). Am J Clin Nutr 2006;83:1369-1379.

12. Lopez-Garcia E, Schulze MB, Fung TT, Meigs JB, Rifai N, Manson JE, Hu FB. Major dietary patterns are related to plasma concentrations of markers of inflammation and endothelial dysfunction. Am J Clin Nutr 2004;80:1029-1035.

13. Fung TT, McCullough ML, Newby PK, Manson JE, Meigs JB, Rifai N, Willett WC, Hu FB. Diet-quality scores and plasma concentrations of markers of inflammation and endothelial dysfunction. Am J Clin Nutr 2005;82:163-173.

14. He K, Liu K, Daviglus ML, Jenny NS, Mayer-Davis E, Jiang R, Steffen L, Siscovick D, Tsai M, Herrington D. Associations of dietary long-chain n-3 polyunsaturated fatty acids and fish with biomarkers of inflammation and endothelial activation (from the Multi-Ethnic Study of Atherosclerosis [MESA]). Am J Cardiol 2009;103:1238-1243.

15. Gao X, Bermudez OI, Tucker KL. Plasma C-reactive protein and homocysteine concentrations are related to frequent fruit and vegetable intake in Hispanic and non-Hispanic white elders. J Nutr 2004;134:913-918.

16. Wannamethee SG, Lowe GD, Rumley A, Bruckdorfer KR, Whincup PH. Associations of vitamin C status, fruit and vegetable intakes, and markers of inflammation and hemostasis. Am J Clin Nutr 2006;83:567-574; quiz 726-727.

17. Calder PC, Ahluwalia N, Brouns F, Buetler T, Clement K, Cunningham K, Esposito K, Jonsson LS, Kolb H, Lansink M, Marcos A, Margioris A, Matusheski N, Nordmann H, O'Brien J, Pugliese G, Rizkalla S, Schalkwijk C, Tuomilehto J, Wärnberg J, Watzl B, Winklhofer-Roob BM. Dietary factors and low-grade inflammation in relation to overweight and obesity. Br J Nutr 2011;106 Suppl 3:S5-78. 
18. Stehouwer CD, Gall MA, Twisk JW, Knudsen E, Emeis JJ, Parving HH. Increased urinary albumin excretion, endothelial dysfunction, and chronic low-grade inflammation in type 2 diabetes: progressive, interrelated, and independently associated with risk of death. Diabetes 2002;51: 1157-1165.

19. Kruijshoop M, Feskens EJ, Blaak EE, de Bruin TW. Validation of capillary glucose measurements to detect glucose intolerance or type 2 diabetes mellitus in the general population. Clin Chim Acta 2004;341:33-40.

20. Du $H$, van der $A D$, van Bakel MM, van der Kallen CJ, Blaak EE, van Greevenbroek MM, Jansen EH, Nijpels G, Stehouwer CD, Dekker JM, Feskens EJ. Glycemic index and glycemic load in relation to food and nutrient intake and metabolic risk factors in a Dutch population. Am J Clin Nutr 2008;87:655-661.

21. Jacobs M, van Greevenbroek MM, van der Kallen CJ, Ferreira I, Blaak EE, Feskens EJ, Jansen EH, Schalkwijk CG, Stehouwer CD. Low-grade inflammation can partly explain the association between the metabolic syndrome and either coronary artery disease or severity of peripheral arterial disease: the CODAM study. Eur J Clin Invest 2009;39:437-444.

22. Jacobs $M$, van Greevenbroek MM, van der Kallen CJ, Ferreira I, Blaak EE, Feskens EJ, Jansen EH, Schalkwijk CG, Stehouwer CD. The association between the metabolic syndrome and peripheral, but not coronary, artery disease is partly mediated by endothelial dysfunction: the CODAM study. Eur J Clin Invest 2010;41:167-175.

23. Thewissen MM, Damoiseaux JG, Duijvestijn AM, van Greevenbroek MM, van der Kallen CJ, Feskens EJ, Blaak EE, Schalkwijk CG, Stehouwer CD, Tervaert JW, Ferreira I. Abdominal Fat Mass Is Associated With Adaptive Immune Activation: The CODAM Study. Obesity (Silver Spring) 2010;19:1690-1698.

24. van Greevenbroek MM, Jacobs M, van der Kallen CJ, Blaak EE, Jansen EH, Schalkwijk CG, Feskens EJ, Stehouwer CD. Human plasma complement C3 is independently associated with coronary heart disease, but only in heavy smokers (the CODAM study). Int J Cardiol 2012;154:158-162.

25. Ocke MC, Bueno-de-Mesquita HB, Goddijn HE, Jansen A, Pols MA, van Staveren WA, Kromhout D. The Dutch EPIC food frequency questionnaire. I. Description of the questionnaire, and relative validity and reproducibility for food groups. Int J Epidemiol 1997;26 Suppl 1:S37-48.

26. Ocke MC, Bueno-de-Mesquita HB, Pols MA, Smit HA, van Staveren WA, Kromhout D. The Dutch EPIC food frequency questionnaire. II. Relative validity and reproducibility for nutrients. Int J Epidemiol 1997;26 Suppl 1:S49-58.

27. NEVO. Nederlands voedingsstoffenbestand, NEVO-tabel 2001. The Hague, The Netherlands: NEVO 2001.

28. van Bussel BC, Henry RM, Schalkwijk CG, Dekker JM, Nijpels G, Stehouwer CD. Low-grade inflammation, but not endothelial dysfunction, is associated with greater carotid stiffness in the elderly: the Hoorn Study. J Hypertens 2012;30:744-752.

29. van Bussel BC, Schouten F, Henry RM, Schalkwijk CG, de Boer MR, Ferreira I, Smulders YM, Twisk JW, Stehouwer CD. Endothelial dysfunction and low-grade inflammation are associated with greater arterial stiffness over a 6-year period. Hypertension 2011;58:588-595.

30. van Bussel BC, Ferreira I, van de Waarenburg MP, van Greevenbroek MM, van der Kallen CJ, Henry RM, Feskens EJ, Stehouwer CD, Schalkwijk CG. Multiple Inflammatory Biomarker Detection in a Prospective Cohort Study: A Cross-Validation between Well-Established Single-Biomarker Techniques and an Electrochemiluminescense-Based Multi-Array Platform. PLoS One 2013;8:e58576.

31. de Valk-de Roo GW, Stehouwer CD, Lambert J, Schalkwijk CG, van der Mooren MJ, Kluft C, Netelenbos C. Plasma homocysteine is weakly correlated with plasma endothelin and von Willebrand factor but not with endothelium-dependent vasodilatation in healthy postmenopausal women. Clin Chem 1999;45:1200-1205.

32. Rothman KJ, Greenland S, Lash TL. Modern Epidemiology. Philadelphia: Lippincott Williams \& Wilkins 2008.

33. Yudkin JS, Stehouwer CD, Emeis JJ, Coppack SW. C-reactive protein in healthy subjects: associations with obesity, insulin resistance, and endothelial dysfunction: a potential role for cytokines originating from adipose tissue? Arterioscler Thromb Vasc Biol 1999;19:972-978.

34. Schram MT, Stehouwer CD. Endothelial dysfunction, cellular adhesion molecules and the metabolic syndrome. Horm Metab Res 2005;37 Suppl 1:49-55.

35. Twisk JWR. Applied Longitudinal Data Analysis for Epidemiology: A Practical Guide. 1st ed. Cambridge, United Kingdom: Cambridge University, 2003. 
36. van Bussel BC, Soedamah-Muthu SS, Henry RM, Schalkwijk CG, Ferreira I, Chaturvedi N, Toeller M, Fuller $\mathrm{JH}$, Stehouwer CD. Unhealthy dietary patterns associated with inflammation and endothelial dysfunction in type 1 diabetes: The EURODIAB study. Nutr Metab Cardiovasc Dis 2013;23:758-764.

37. van Aerde MA, Soedamah-Muthu SS, Geleijnse JM, Snijder MB, Nijpels G, Stehouwer CD, Dekker JM. Dairy intake in relation to cardiovascular disease mortality and all-cause mortality: the Hoorn Study. Eur J Nutr 2013;52:609-616.

38. van de Laar RJ, Stehouwer CD, van Bussel BC, Te Velde SJ, Prins MH, Twisk JW, Ferreira I. Lower lifetime dietary fiber intake is associated with carotid artery stiffness: the Amsterdam Growth and Health Longitudinal Study. Am J Clin Nutr 2012;96:14-23.

39. Brown AA, Hu FB. Dietary modulation of endothelial function: implications for cardiovascular disease. Am J Clin Nutr 2001;73:673-686.

40. Hall WL. Dietary saturated and unsaturated fats as determinants of blood pressure and vascular function. Nutr Res Rev 2009;22:18-38.

41. Bau PF, Bau CH, Rosito GA, Manfroi WC, Fuchs FD. Alcohol consumption, cardiovascular health, and endothelial function markers. Alcohol 2007;41:479-488.

42. van Bussel BC, Henry RM, Schalkwijk CG, Ferreira I, Feskens EJ, Streppel MT, Smulders YM, Twisk JW, Stehouwer $\mathrm{CD}$. Fish consumption in healthy adults is associated with decreased circulating biomarkers of endothelial dysfunction and inflammation during a 6-year follow-up. J Nutr 2011;141: 1719-1725.

43. Sacanella E, Vazquez-Agell M, Mena MP, Antunez E, Fernandez-Sola J, Nicolas JM, Lamuela-Raventos RM, Ros E, Estruch R. Down-regulation of adhesion molecules and other inflammatory biomarkers after moderate wine consumption in healthy women: a randomized trial. Am J Clin Nutr 2007;86:1463-1469.

44. van de Laar RJ, Stehouwer CD, van Bussel BC, Prins MH, Twisk JW, Ferreira I. Adherence to a Mediterranean dietary pattern in early life is associated with lower arterial stiffness in adulthood: the Amsterdam Growth and Health Longitudinal Study. J Intern Med 2012.

45. Laurent S, Boutouyrie P, Asmar R, Gautier I, Laloux B, Guize L, Ducimetiere P, Benetos A. Aortic stiffness is an independent predictor of all-cause and cardiovascular mortality in hypertensive patients. Hypertension 2001;37:1236-1241.

46. Yang EY, Chambless L, Sharrett AR, Virani SS, Liu X, Tang Z, Boerwinkle E, Ballantyne CM, Nambi V. Carotid arterial wall characteristics are associated with incident ischemic stroke but not coronary heart disease in the Atherosclerosis Risk in Communities (ARIC) study. Stroke 2012;43:103-108.

47. Nagel G, Zoller D, Ruf T, Rohrmann S, Linseisen J. Long-term reproducibility of a food-frequency questionnaire and dietary changes in the European Prospective Investigation into Cancer and Nutrition (EPIC)-Heidelberg cohort. Br J Nutr 2007;98:194-200.

48. Aird WC. Phenotypic heterogeneity of the endothelium: I. Structure, function, and mechanisms. Circ Res 2007;100:158-173.

49. Aird WC. Phenotypic heterogeneity of the endothelium: II. Representative vascular beds. Circ Res 2007;100:174-190.

50. Deanfield J, Donald A, Ferri C, Giannattasio C, Halcox J, Halligan S, Lerman A, Mancia G, Oliver JJ, Pessina AC, Rizzoni D, Rossi GP, Salvetti A, Schiffrin EL, Taddei S, Webb DJ. Endothelial function and dysfunction. Part I: Methodological issues for assessment in the different vascular beds: a statement by the Working Group on Endothelin and Endothelial Factors of the European Society of Hypertension. J Hypertens 2005;23:7-17.

51. Willett WC. Nutritional Epidemiology. 2th ed. New York, NY: Oxford University Press, 1998. 


\section{Appendix 6}

\section{The association between changes in food consumption and changes in endothelial dysfunction and low-grade inflammation}

To investigate whether the longitudinal associations (by GEE) were primarily determined by the between- or the within-subjects associations over the 7-year study period, we investigated, with the use of linear regression analyses, the extent to which changes (i.e. within-subjects) in dietary variables were associated with changes in biomarkers of endothelial dysfunction or low-grade inflammation. Changes were calculated by subtracting the values of variables obtained in CODAM- 2 from those obtained in CODAM-1 [these analyses were confined to complete cases $(n=421)$ ]. Changes in diet were not significantly associated with changes in the overall endothelial dysfunction score, except for a positive association for high-fat dairy product consumption $[\beta(95 \% \mathrm{Cl}) 0.067(0.006 ; 0.128)]$. Changes in diet were not significantly associated with changes in the overall low-grade inflammation score, except for a positive association for liquor consumption [0.047 $(0.009 ; 0.085)]$, a positive association for total dairy product consumption $[0.040(0.006 ; 0.073)]$ and a positive association for high-fat dairy product consumption [0.085 (0.004;0.166)] (Table S6.1).

Overall, this suggests that the reported associations (by GEE) were primarily determined by the between-subject association over the 7-year study period. 
Table S6.1 Associations between changes in food consumption and changes in endothelial dysfunction and low-grade inflammation over 7 years.

\begin{tabular}{|c|c|c|c|c|}
\hline & \multicolumn{2}{|c|}{ Endothelial dysfunction score } & \multicolumn{2}{|c|}{$\begin{array}{c}\text { Low-grade inflammation } \\
\text { score }\end{array}$} \\
\hline & $\beta$ & $95 \% \mathrm{Cl}$ & $\beta$ & $95 \% \mathrm{Cl}$ \\
\hline Total fish, $100 \mathrm{~g} / \mathrm{wk}$ & -0.022 & $-0.063 ; 0.018$ & 0.005 & $-0.049 ; 0.058$ \\
\hline Fat fish, $100 \mathrm{~g} / \mathrm{wk}$ & -0.009 & $-0.120 ; 0.102$ & 0.064 & $-0.082 ; 0.210$ \\
\hline Lean fish, $100 \mathrm{~g} / \mathrm{wk}$ & -0.041 & $-0.103 ; 0.020$ & -0.009 & $-0.090 ; 0.073$ \\
\hline Total vegetables, $100 \mathrm{~g} / \mathrm{d}$ & -0.045 & $-0.121 ; 0.031$ & 0.027 & $-0.073 ; 0.128$ \\
\hline Raw vegetables, $100 \mathrm{~g} / \mathrm{d}$ & -0.085 & $-0.249 ; 0.080$ & 0.041 & $-0.176 ; 0.258$ \\
\hline Cooked vegetables, $100 \mathrm{~g} / \mathrm{d}$ & -0.050 & $-0.146 ; 0.046$ & 0.010 & $-0.118 ; 0.137$ \\
\hline Total fruit, $100 \mathrm{~g} / \mathrm{d}$ & 0.012 & $-0.018 ; 0.042$ & 0.004 & $-0.036 ; 0.044$ \\
\hline Fresh fruit, $100 \mathrm{~g} / \mathrm{d}$ & 0.008 & $-0.028 ; 0.045$ & -0.007 & $-0.055 ; 0.040$ \\
\hline Fruit juice, $100 \mathrm{ml} / \mathrm{d}$ & 0.017 & $-0.034 ; 0.067$ & 0.013 & $-0.054 ; 0.079$ \\
\hline Total alcohol-containing beverages, $10 \mathrm{~g} / \mathrm{d}$ & 0.008 & $-0.049 ; 0.065$ & -0.022 & $-0.097 ; 0.052$ \\
\hline Wine, $100 \mathrm{ml} / \mathrm{wk}$ & -0.004 & $-0.014 ; 0.007$ & -0.012 & $-0.026 ; 0.001$ \\
\hline Beer, $100 \mathrm{ml} / \mathrm{wk}$ & -0.001 & $-0.005 ; 0.003$ & -0.002 & $-0.007 ; 0.003$ \\
\hline Liquor, $100 \mathrm{ml} / \mathrm{wk}$ & 0.023 & $-0.006 ; 0.052$ & 0.046 & $0.008 ; 0.084 *$ \\
\hline Total dairy products, $100 \mathrm{~g} / \mathrm{d}$ & 0.017 & $-0.008 ; 0.042$ & 0.040 & $0.006 ; 0.073 *$ \\
\hline Low-fat dairy products, $100 \mathrm{~g} / \mathrm{d}$ & 0.009 & $-0.022 ; 0.039$ & 0.037 & $-0.003 ; 0.077$ \\
\hline High-fat dairy products, $100 \mathrm{~g} / \mathrm{d}$ & 0.067 & $0.006 ; 0.128 *$ & 0.086 & $0.005 ; 0.166 *$ \\
\hline
\end{tabular}

$\beta$, regression coefficient as obtained from linear regression analysis indicates the change in the overall endothelial dysfunction and low-grade inflammation scores per (specified) unit increase in fish, vegetable, fruit, alcohol-containing beverage and dairy product consumption; $95 \% \mathrm{Cl}, 95 \%$ confidence interval; * p-value $<0.05$; complete cases $n=421$. All data are adjusted for sex, age at baseline and changes in age, energy intake, glucose metabolism status, body mass index, physical activity, alcohol categories and current smoking. 


\section{Chapter \\ 7}

Unhealthy dietary patterns associated with inflammation and endothelial dysfunction in type 1 diabetes: the EURODIAB Study

BCT van Bussel, SS Soedamah-Muthu, RMA Henry, CG Schalkwijk, I Ferreira, N Chaturvedi, M Toeller, JH Fuller, CDA Stehouwer; EURODIAB Prospective Complications Study Group

Nutr Metab Cardiovasc Dis. 2013;23:758-764

Reprinted with permission of the publisher 


\section{Abstract}

\section{Background and aims}

A healthy diet has been inversely associated with endothelial dysfunction (ED) and low-grade inflammation (LGI). We investigated the association between nutrient consumption and biomarkers of ED and LGI in type 1 diabetes.

\section{Methods and results}

We investigated 491 individuals. Nutrient consumption and lifestyle risk factors were measured in 1989 and 1997. Biomarkers of ED (von Willebrand factor, soluble vascular cell adhesion molecule-1 and soluble endothelial selectin) and LGI (C-reactive protein, interleukin 6 and tumour necrosis factor $\alpha$ ) were measured in 1997 and averaged into Z-scores. The nutrient residual method was used to adjust individual nutrient intake for energy intake. Data were analyzed with generalized estimation equations. We report increments/decrements in nutrient consumption, averaged over time, per +1 standard deviation (SD) of 1997 ED or LGI Z-scores, after adjustment for sex, age, duration of diabetes, investigation centre, body mass index, energy intake, smoking behaviour, alcohol consumption, and each of the other nutrients. One $S D$ elevation in ED Z-score was associated with a diet lower in fibre $[\beta(95 \% \mathrm{Cl})$; $-0.09(-0.18$;$0.004)]$, polyunsaturated fat $[-0.18(-0.31 ;-0.05)]$ and vegetable protein $[-0.10(-0.20 ;-0.001)]$. For the LGI Z-score results showed associations with fibre [-0.09(-0.17;-0.01)], polyunsaturated fat [$0.14(-0.24 ;-0.03)]$ and cholesterol $[0.10(0.01 ; 0.18))$.

\section{Conclusion}

In type 1 diabetes, consumption of less fibre, polyunsaturated fat and vegetable protein, and more cholesterol over the study period was associated with more ED and LGI. Following dietary guidelines in type 1 diabetes may reduce cardiovascular disease risk by favourably affecting ED and LGI. 


\section{Introduction}

Diet is one of the more easily modifiable cardiovascular disease (CVD) risk factors ${ }^{1}$ as it has been shown that a healthy diet is associated with a reduced incidence of CVD. ${ }^{2-4}$ This may be explained, at least partly, by the dietary modification of endothelial dysfunction and low-grade inflammation. ${ }^{5,6}$

The American Diabetes Association (ADA) and the Diabetes and Nutrition Study Group (DNSG) of the European Association for the Study of Diabetes (EASD) advise individuals with type 1 diabetes to increase their consumption of fibre and to reduce their consumption of saturated fat and dietary cholesterol in order to prevent or delay the onset of CVD and other complications of diabetes. ${ }^{7,8}$

We recently showed that, in healthy adults, the consumption of long-chain polyunsaturated fatty acids was associated with decreased endothelial dysfunction and low-grade inflammation scores during a 6-year follow-up. ${ }^{9}$ However, it is unknown whether diet affects endothelial dysfunction and low-grade inflammation in individuals with type 1 diabetes.

We, therefore, hypothesized that the consumption of fibre, carbohydrate, fat, cholesterol and protein is associated with plasma biomarkers of endothelial dysfunction and low-grade inflammation.

Previous studies on diet and markers of endothelial dysfunction and low-grade inflammation in the general population ${ }^{10}$ have relied on cross-sectional associations, whereas dietary practices over time offer better precision. ${ }^{11}$

Therefore, in the present study we have investigated nutrient exposure at two timepoints, i.e. at the start and at the end of an 8-year study period. We investigated the associations between the consumptions of fibre, carbohydrate, fat, cholesterol and protein on average over an 8-year study period and plasma biomarkers of endothelial dysfunction and low-grade inflammation at the end of this period. More specifically, we investigated whether average nutrient consumption of 1989 and 1997 was associated with markers of a) endothelial dysfunction and b) low-grade inflammation in 1997. In addition, we investigated whether any such associations were independent of other lifestyle risk factors and other nutrients. Nutrients were investigated independent of energy intake ${ }^{12}$ to estimate each nutrient's individual effect on the above associations.

\section{Methods}

\section{Study population}

For the present investigation we used data from the EURODIAB Prospective Complications Study, which is a follow-up of the EURODIAB Type 1 Diabetes Complications Study. ${ }^{13}$ Baseline investigations (from 1988 to 1991, further referred to as 1989) were performed on 3250 men and women with type 1 diabetes aged 
between 15 and 60 years, from 16 European countries, who were stratified according to sex, age and duration of diabetes. Type 1 diabetes was clinically defined as diagnosis made before the age of 36 years, with a continuous need for insulin therapy within 1 year of diagnosis.

Of the original 3250 individuals, 1880 individuals returned for a follow-up examination approximately 8 years later (further referred to as 1997). ${ }^{14}$

At this follow-up examination a nested case-control study design was employed to maximize statistical efficiency (details have been described elsewhere). ${ }^{15,16}$ Briefly, cases were selected as those with the greatest vascular complication burden $(n=348)$, and controls were selected as those free of any complications (i.e. with no evidence of CVD, albuminuria and retinopathy) $(n=195){ }^{15,16}$ In plasma samples of these individuals, biomarkers of endothelial dysfunction [soluble endothelial selectin (sEselectin) and soluble vascular cell adhesion molecule-1 (sVCAM-1)], and of low-grade inflammation [C-reactive protein (CRP), interleukin 6 (IL-6) and tumour necrosis factor $\alpha($ TNF- $\alpha)$ ] had already been determined. ${ }^{16}$ Additionally, we added von Willebrand factor (vWf), a plasma biomarker of endothelial dysfunction, to this set of biomarkers. For the present study, data on nutrient consumption, plasma biomarkers of endothelial dysfunction and low-grade inflammation, and lifestyle risk factors were available in 491 individuals. All individuals gave informed consent and the study was approved by the local ethics committees. ${ }^{17}$

\section{Dietary exposure assessment}

In 1989 and 1997, a standardized three day dietary record was employed for dietary assessment, as previously described. ${ }^{17,18}$ Briefly, local dieticians instructed individuals on how to record all foods and beverages consumed over 3 days ( 2 workdays and a Sunday) with the help of standardized food portion sizes. The local dietician reviewed each completed record with each individual. Then, with the use of a centrally prepared EURODIAB food list, the records were analyzed to quantify the amounts of fibre (soluble and insoluble), carbohydrate, fat (saturated, monounsaturated and polyunsaturated) cholesterol, protein (animal and vegetable), alcohol and total energy. ${ }^{17,19,20}$ Alcohol consumption was categorized into none, low ( $\leq$ median: $7.8 \mathrm{~g} / \mathrm{d}$ in 1989 and $10.0 \mathrm{~g} / \mathrm{d}$ in 1997) and high consumers (> median).

\section{Assessment of endothelial dysfunction and low-grade inflammation}

Plasma biomarkers of endothelial dysfunction (vWf, sVCAM-1 and sE-selectin) were measured by ELISA. Briefly, vWf was determined in citrated plasma using a rabbit antibody as a catching antibody and a peroxidase-conjugate rabbit antibody as detecting antibody ${ }^{21}$ (Dako, Glostrup, Denmark). Concentrations were expressed as a percentage of vWf detected in pooled citrated plasma of healthy volunteers. The intra- and inter-assay coefficients of variation for vWf were 3.5 and $4.7 \%$. sVCAM-1 and $s E$-selectin were measured with the use of commercially available ELISA kits (R\&D 
Systems, Oxford, UK). The intra- and inter-assay coefficients of variation for SVCAM-1 were 4.0 and $9.1 \%$, and for $\mathrm{sE}$-selectin, 2.1 and $3.1 \%$.

Plasma biomarkers of low-grade inflammation (CRP, IL- 6 and TNF- $\alpha$ ) were measured with a highly sensitive in-house ELISA (CRP) ${ }^{16}$ and with the use of commercially available ELISA kits (IL-6 and TNF- $\alpha$, R\&D Systems). ${ }^{16}$ The intra- and inter-assay coefficients of variation were 3.9 and $8.7 \%, 4.5$ and $9.0 \%$, and 7.3 and $8.5 \%$, respectively.

\section{Other measurements}

In 1989 and 1997, height, weight, HbA1c and blood pressure were measured as previously described ${ }^{14}$ and information on duration of diabetes, frequency of insulin use and individuals' smoking behaviour (whether they smoked currently, or more than 5 cigarettes/day in the past, and whether they smoked cigar or pipe) was obtained by questionnaire. ${ }^{13,22}$ We calculated body mass index (BMI); and smoking behaviour was dichotomized into smokers (including both ever and current smokers) and nonsmokers.

\section{Statistical analyses}

All statistical analyses were performed with STATA software package version 9.2 (STATA Corp). Variables with a skewed distribution (polyunsaturated fat and cholesterol consumption, CRP, IL-6, TNF- $\alpha$ ) were log-transformed in order to meet normality criteria.

For reasons of statistical efficiency and to reduce the influences of the biological variability of each measure ${ }^{23}$, an overall Z-score was determined for both endothelial dysfunction and low-grade inflammation, according to predefined clusters of conceptually related biomarkers (please see Appendix 7 for details). ${ }^{23,24}$

Nutrient consumption was investigated independent of energy intake using the nutrient residual method (please see Appendix 7 for details) ${ }^{12}$, unless otherwise specified.

To investigate the association between the average consumptions of nutrients (residuals) over the 8-year study period, and the overall endothelial dysfunction and low-grade inflammation Z-scores (higher scores indicating more dysfunction) at the end of this period, we used generalized estimating equations (GEE). The use of GEE as a method of longitudinal data analysis allows for adjustment for the correlation between repeated observations of nutrient consumption and covariates taken in the same individual (for the current analysis: nutrient intake and covariates were entered for 1989 and 1997). ${ }^{25}$ In addition, GEE has the advantage of handling missing data robustly. ${ }^{25}$ The regression coefficient represents the increment or decrement in nutrient consumption averaged over the time per +1 SD in the 1997 overall endothelial dysfunction or low-grade inflammation Z-scores (higher scores indicating worse function). ${ }^{25-27}$ 
First, we investigated the increments/decrements in consumption of fibre (soluble and insoluble), carbohydrate, fat (saturated, monounsaturated and polyunsaturated), cholesterol and protein (animal and vegetable) per +1 SD of the endothelial dysfunction and low-grade inflammation overall Z-scores (models 1). Second, these nutritional estimates were adjusted for sex, age, duration of diabetes, investigation centre, BMI, total energy intake, smoking behaviour, alcohol consumption and each of the other nutrients in 1989 and 1997 (models 2).

Data are presented as mean $( \pm S D$ ), median (interquartile range) for skewed variables or as percentages and regression coefficients $(\beta)$ with their $95 \%$ confidence intervals $(95 \% \mathrm{Cl})$. In all GEE analyses, an exchangeable correlation structure was assumed. A two-sided $p$-value $\leq 0.05$ was considered statistically significant.

\section{Results}

Table 7.1 shows the characteristics of the study population (48.7\% women). In 1989 the mean ( \pm SD) age and duration of diabetes were $32.6 \pm 10.1$ and $14.4 \pm 9.6$ years, respectively. Between 1989 and 1997 nutrient consumption remained stable (please see Appendix 7, Table S7.1).

Table 7.1 Characteristics of the study population. ${ }^{\text {a }}$

\begin{tabular}{|c|c|c|}
\hline Clinical characteristics & baseline 1989 & follow-up 1997 \\
\hline Age, years & $32.6 \pm 10.1$ & $39.8 \pm 10.1$ \\
\hline Female, \% & 48.7 & 48.7 \\
\hline Diabetes duration, years & $14.4 \pm 9.6$ & $21.6 \pm 9.6$ \\
\hline $\mathrm{BMI}, \mathrm{kg} / \mathrm{m}^{2}$ & $23.4 \pm 2.8^{b}$ & $24.5 \pm 3.2^{c}$ \\
\hline Smoking (ever + current), \% & $49.5^{b}$ & 59.9 \\
\hline Alcohol (non), \% & 48.5 & 43.5 \\
\hline Alcohol (low), \% & 25.7 & 28.1 \\
\hline Alcohol (high), \% & 25.9 & 28.4 \\
\hline \multicolumn{3}{|l|}{ Plasma biomarkers } \\
\hline von Willebrand factor, \% & - & $134.3 \pm 61.7$ \\
\hline Soluble E-selectin, $\mathrm{ng} / \mathrm{ml}$ & - & $33.9 \pm 14.4$ \\
\hline Soluble vascular cell adhesion molecule- $1, \mathrm{ng} / \mathrm{ml}$ & - & $414.6 \pm 133.9$ \\
\hline C-reactive protein, $\mathrm{mg} / \mathrm{l}$ & - & $1.1(0.4-2.6)$ \\
\hline Interleukin 6, pg/ml & - & $1.9(1.2-3.3)$ \\
\hline Tumour necrosis factor $\alpha, \mathrm{pg} / \mathrm{ml}$ & - & $2.8(2.1-3.6)$ \\
\hline \multicolumn{3}{|l|}{ Dietary characteristics } \\
\hline Energy intake, $\mathrm{kJ} / \mathrm{d}$ & $10079 \pm 2950$ & $9699 \pm 2728^{d}$ \\
\hline Total fibre, g/d & $19 \pm 8$ & $19 \pm 8^{d}$ \\
\hline Soluble fibre, $\mathrm{g} / \mathrm{d}$ & $6 \pm 2$ & $6 \pm 3^{d}$ \\
\hline Insoluble fibre, $\mathrm{g} / \mathrm{d}$ & $13 \pm 5$ & $13 \pm 6^{d}$ \\
\hline Polyunsaturated fat, g/d & $14(9-21)$ & $13(10-17)^{d}$ \\
\hline Cholesterol, mg/d & $335(228-460)$ & $309(222-403)^{d}$ \\
\hline Vegetable protein, $\mathrm{g} / \mathrm{d}$ & $30 \pm 11$ & $29 \pm 10^{d}$ \\
\hline
\end{tabular}

${ }^{a}$ Data are presented as means \pm standard deviation, median (interquartile range) or percentages as appropriate $(n=491) ;^{b}(n=486) ;^{c}(n=488) ;^{d}(n=324)$. 


\section{Nutrient consumption in relation to endothelial dysfunction}

A one SD elevation in endothelial dysfunction Z-score was associated with a diet lower in fibre $[\beta(95 \% \mathrm{Cl})-0.13(-0.25 ;-0.01), p=0.028]$, both soluble $[-0.15(-0.27 ;-0.04)$, $p=0.010]$ and insoluble $[-0.13(-0.25 ;-0.01), p=0.034$ (Table 7.2)]. The endothelial dysfunction Z-score was also inversely associated with polyunsaturated fat and vegetable protein consumption (Table 7.2 ). These associations were only marginally attenuated on multivariate adjustment.

No associations between the consumption of carbohydrate, (saturated or monounsaturated) fat, cholesterol or (animal) protein and the endothelial dysfunction Z-score were observed (please see Appendix 7, Table S7.2).

Table 7.2 Nutrient consumption over time per +1 standard deviation of the endothelial dysfunction or low-grade inflammation Z-scores.

\begin{tabular}{lcc}
\hline Nutrients & \multicolumn{1}{c}{$\beta^{\mathrm{a}}(95 \% \mathrm{Cl})$} & \multicolumn{1}{c}{ Low-grade inflammation } \\
\cline { 2 - 3 } & $-0.13(-0.25 ;-0.01)^{*}$ & $\beta^{a}(95 \% \mathrm{Cl})$ \\
\hline Total fibre $^{\mathrm{b}}$ & $-0.09(-0.18 ;-0.004)^{*}$ & $-0.11(-0.22 ;-0.01)^{*}$ \\
Total fibre $^{\mathrm{c}}$ & $-0.15(-0.27 ;-0.04)^{*}$ & $-0.09(-0.17 ;-0.01)^{*}$ \\
Soluble fibre $^{\mathrm{b}}$ & $-0.09(-0.18 ;-0.01)^{*}$ & $-0.14(-0.24 ;-0.04)^{\dagger}$ \\
Soluble fibre $^{\mathrm{c}}$ & $-0.13(-0.25 ;-0.01)^{*}$ & $-0.11(-0.18 ;-0.03){ }^{\dagger}$ \\
Insoluble fibre $^{\mathrm{b}}$ & $-0.09(-0.18 ;-0.003)^{*}$ & $-0.10(-0.21 ; 0.01)$ \\
Insoluble fibre $^{\mathrm{c}}$ & $-0.12(-0.25 ; 0.02)$ & $-0.08(-0.16 ; 0.004)$ \\
Polyunsaturated fat $^{\mathrm{b}}$ & $-0.18(-0.31 ;-0.05) \dagger$ & $-0.06(-0.17 ; 0.06)$ \\
Polyunsaturated fat $^{\mathrm{d}}$ & $0.09(-0.04 ; 0.22)$ & $-0.14(-0.24 ;-0.03)^{*}$ \\
Cholesterol $^{\mathrm{b}}$ & $0.08(-0.02 ; 0.18)$ & $0.14(0.03 ; 0.25)^{*}$ \\
Cholesterol $^{\mathrm{e}}$ & $-0.15(-0.30 ;-0.01)^{*}$ & $0.10(0.01 ; 0.18)^{*}$ \\
Vegetable protein $^{\mathrm{b}}$ & $-0.10(-0.20 ;-0.001)^{*}$ & $-0.13(-0.25 ;-0.01)^{*}$ \\
Vegetable protein $^{\mathrm{f}}$ & & $-0.06(-0.14 ; 0.02)$ \\
\hline
\end{tabular}

${ }^{\text {a }} B(95 \% \mathrm{Cl})$ : indicates increments/decrements in nutrient consumption over time per +1 standard deviation of the endothelial dysfunction or low-grade inflammation Z-scores; ${ }^{*} p \leq 0.05,+p \leq 0.01$; energy-adjusted nutrient residuals in arbitrary units; $n=491 ;{ }^{b}$ Model 1: crude; ${ }^{c}$ Model 2: adjusted for sex, age, duration of diabetes, investigation centre, BMI, energy intake, smoking behaviour, alcohol, fat, protein, cholesterol and carbohydrate consumption in 1989 and 1997; ' Model 2: adjusted for sex, age, duration of diabetes, investigation centre, BMI, energy intake, smoking behaviour, alcohol, carbohydrate, fibre, cholesterol and

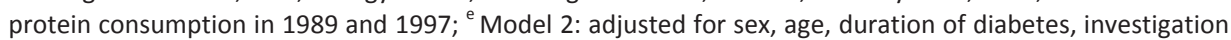
centre, BMI, energy intake, smoking behaviour, alcohol, carbohydrate, fibre, fat and protein consumption in 1989 and 1997; ${ }^{f}$ Model 2: adjusted for sex, age, duration of diabetes, investigation centre, BMI, energy intake, smoking behaviour, alcohol, carbohydrate, fibre, cholesterol and fat consumption in 1989 and 1997.

\section{Nutrient consumption in relation to low-grade inflammation}

A one SD elevation in low-grade inflammation Z-score was associated with a diet lower in fibre $[-0.11(-0.22 ;-0.01), p=0.031]$ and soluble fibre $[-0.14(-0.24 ;-0.04)$, $\mathrm{p}=0.005$ (Table 7.2)]. The low-grade inflammation Z-score was also inversely associated with polyunsaturated fat and vegetable protein consumption, and positively with cholesterol consumption (Table 7.2). These associations were only 
marginally attenuated on multivariate adjustment, and only lost statistical significance for vegetable protein.

No associations between the consumption of insoluble fibre, carbohydrate, (saturated or monounsaturated) fat or (animal) protein and the low-grade inflammation Z-score were observed (please see Appendix 7, Table 57.2).

Additional adjustment for several (cardiovascular) co-morbidities, medication use, educational status and physical activity did not change the results. Alternative analyses with absolute measures of dietary intake or individual biomarkers (Figure 7.1) or with changes in nutrient consumption over time overall gave results similar to those presented here (please see Appendix 7 for details).
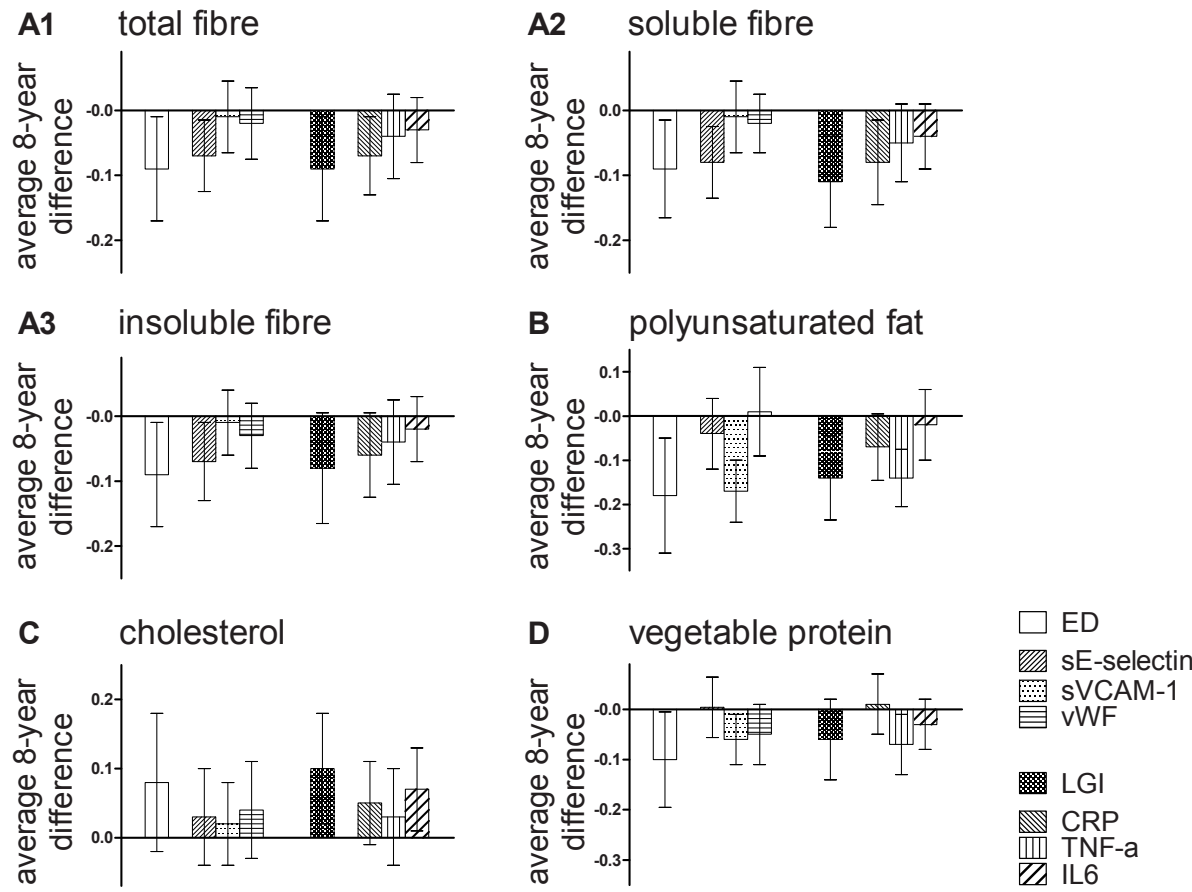

Figure 7.1 Biomarkers as a function of diet.

Bars represent increments/decrements in nutrient consumption averaged over two data collections (1989 and 1997), per +1 standard deviation of the overall endothelial dysfunction (ED), the overall low-grade inflammation (LGI) or the individual biomarker Z-scores, after adjustment for sex, age, duration of diabetes, investigation centre, BMI, energy intake, smoking behaviour, alcohol consumption and each of the other nutrients; whiskers indicate $95 \%$ confidence intervals; $n=491$. 


\section{Discussion}

The present study had three main findings. First, individuals with greater endothelial dysfunction consumed less fibre (both soluble and insoluble fibre), less polyunsaturated fat and less vegetable protein over the 8-year study period, whereas no associations were found for carbohydrate, (other types of) fat, cholesterol or (animal) protein. Second, individuals with greater low-grade inflammation consumed less fibre, less soluble fibre, less polyunsaturated fat, and more cholesterol over the 8-year study period, whereas no associations were found for insoluble fibre, carbohydrate, (other types of) fat or (any type of) protein. Third, these results were independent of sex, age, duration of diabetes, investigation centre, BMI, energy intake, smoking behaviour, alcohol consumption and each of the other nutrients.

The present investigation was comprehensive and supports the dietary ADA and DNSG of the EASD guidelines on the consumption of fibre and cholesterol for individuals with type 1 diabetes ${ }^{7,8}$, as the consumption of more fibre and less cholesterol was associated with more favourable overall Z-scores for endothelial dysfunction and/or low-grade inflammation. Higher biomarker concentrations are associated with (incident) CVD. ${ }^{24,28,29}$ The present results are consistent with those of Esposito et al. and Lopes-Garcia et al. ${ }^{6,10}$, which suggest that a healthy diet may modify endothelial dysfunction and low-grade inflammation and can thus prevent or delay the onset of CVD.

The ADA and DNSG of the EASD recommendations do not specifically advise on the source of protein due to lack of scientific evidence. ${ }^{7,8}$ However, the present investigation shows that the consumption of vegetable protein was associated with a more favourable endothelial dysfunction Z-score and therefore may provide evidence that vegetable-derived food sources may provide a healthier source of protein than animal-derived food sources.

Current evidence-based nutritional recommendations emphasize a restriction of saturated fat intake and preference of fat sources which provide unsaturated fatty acids. The present investigation shows that the consumption of polyunsaturated fat was associated with more favourable overall Z-scores for endothelial dysfunction and low-grade inflammation. This may be related to the long-chain polyunsaturated fatty acids EPA and DHA, which have favourable effects on endothelial dysfunction and low-grade inflammation. ${ }^{9,30}$ Unfortunately, the present data did not allow us to investigate this issue because the EURODIAB food list did not include information on specific types of polyunsaturated fat.

The present investigation cannot fully exclude confounding by indication, i.e. individuals with more severe disease received more dietary advice over the 8-year study period, with the result that these individuals improved their diet to a greater extent, compared to individuals with less severe disease. However, we consider such confounding highly unlikely, as the results did not change when complication status was taken into account. Second, we did not observe any associations between 
changes in nutrient consumption over time and either endothelial dysfunction or lowgrade inflammation, except that individuals who decreased their polyunsaturated fat consumption had lower endothelial dysfunction and low-grade inflammation scores. The results of this secondary analysis should, however, be interpreted with caution, because a biological explanation for this finding is not readily at hand and we cannot exclude the play of chance. Taken together, these results suggest that nutrient consumption is stable in relation to endothelial dysfunction and low-grade inflammation. This is in line with recent data from the EPIC-Heidelberg cohort, which have shown that dietary habits remain stable over the $3^{\text {rd, }} 4^{\text {th }}$ and $5^{\text {th }}$ decades of life. ${ }^{11}$ With regard to the individual biomarkers, we show that an 8-year average consumption of less fibre (both soluble and insoluble fibre) was associated with greater sE-selectin and CRP concentrations, whereas less polyunsaturated fat and vegetable protein were associated with greater SVCAM-1 and TNF- $\alpha$ concentrations, and more cholesterol was associated with a greater IL-6 concentration (Figure 7.1). Thus, we cannot exclude that each nutrient may affect endothelial dysfunction and/or low-grade inflammation differentially. Importantly, the results for the overall Z-score consistently showed that individuals with greater scores had a less favourable diet.

To our knowledge the present study is the first to report on diet and biomarkers of endothelial dysfunction and low-grade inflammation in individuals with type 1 diabetes. Its strengths are: (1) the nested case-control design, which maximizes statistical efficiency due to the fact that this design includes individuals at both ends of the vascular complication spectrum (i.e. those with the greatest vascular complication burden and those who are free of complications); (2) the highly standardized dietary exposure assessments at two time-points, which allows studying diet over an 8-year study period, which additionally reduces the chance of a type 2 error by inclusion of both dietary assessments and potential confounders in 1989 and 1997 into one analysis.

The present study has some limitations. First, intrinsic to an overall Z-score is the assumption that each biomarker in the Z-score carries similar weight. It is however unknown whether this is the case for each individual biomarker in our Z-score with regard to cardiovascular disease risk. The chosen approach may therefore not reflect pathophysiology optimally. Second, we cannot fully exclude that other health behaviours might have affected our findings. Nevertheless, the associations remained unchanged after additional adjustment for the lifestyle risk factors investigated including physical activity, medication use and educational status. Third, biomarkers were assessed at one time-point only (follow-up examination of 1997). This single assessment at one time-point, as compared to multiple assessments over time, is subject to greater biological variability, for instance, due to influence of any (intercurrent) disease on biomarker level. Yet, when we repeated the analyses without those individuals whose biomarker levels were outside the arbitrary +2SD range, the results were not materially altered (data not shown). Finally, we cannot fully exclude that reverse causation may have occurred; i.e. that individuals with 
greater endothelial dysfunction or low-grade inflammation in 1997 might also be those with greater endothelial dysfunction and low-grade inflammation at earlier time-points and consequently might have been more prone to consume an unfavourable diet. However, we consider this scenario highly unlikely as individuals are unaware of their endothelial dysfunction and low-grade inflammation as both traits have an asymptomatic character.

In conclusion, in individuals with type 1 diabetes, the consumption of less fibre, less polyunsaturated fat, less vegetable protein and more cholesterol over the 8-year study period was associated with more endothelial dysfunction and low-grade inflammation. These results support the ADA and the DNSG of the EASD dietary recommendations to prevent or delay the onset of CVD and other complications of diabetes. 


\section{References}

1. Lichtenstein $A H$, Appel $\amalg$, Brands $M$, Carnethon $M$, Daniels $S$, Franch HA, Franklin B, Kris-Etherton $P$, Harris WS, Howard B, Karanja N, Lefevre M, Rudel L, Sacks F, Van Horn L, Winston M, Wylie-Rosett J. Diet and lifestyle recommendations revision 2006: a scientific statement from the American Heart Association Nutrition Committee. Circulation. 2006; 114:82-96.

2. Hu FB, Rimm EB, Stampfer MJ, Ascherio A, Spiegelman D, Willett WC. Prospective study of major dietary patterns and risk of coronary heart disease in men. Am J Clin Nutr. 2000; 72:912-921.

3. Knoops KT, de Groot LC, Kromhout D, Perrin AE, Moreiras-Varela O, Menotti A, van Staveren WA. Mediterranean diet, lifestyle factors, and 10-year mortality in elderly European men and women: the HALE project. JAMA. 2004; 292:1433-1439.

4. Hodge AM, English DR, Itsiopoulos C, O'Dea K, Giles GG. Does a Mediterranean diet reduce the mortality risk associated with diabetes: evidence from the Melbourne Collaborative Cohort Study. Nutr Metab Cardiovasc Dis. 2011; 21:733-739.

5. Ross R. Atherosclerosis--an inflammatory disease. N Engl J Med. 1999; 340:115-126.

6. Esposito K, Marfella R, Ciotola M, Di Palo C, Giugliano F, Giugliano G, D'Armiento M, D'Andrea F, Giugliano D. Effect of a mediterranean-style diet on endothelial dysfunction and markers of vascular inflammation in the metabolic syndrome: a randomized trial. JAMA. 2004; 292:1440-1446.

7. Bantle JP, Wylie-Rosett J, Albright AL, Apovian CM, Clark NG, Franz MJ, Hoogwerf BJ, Lichtenstein AH, Mayer-Davis E, Mooradian AD, Wheeler ML. Nutrition recommendations and interventions for diabetes: a position statement of the American Diabetes Association. Diabetes Care. 2008; 31 Suppl 1: S61-78.

8. Mann JI, De Leeuw I, Hermansen K, Karamanos B, Karlström B, Katsilambros N, Riccardi G, Rivellese AA, Rizkalla S, Slama G, Toeller M, Uusitupa M, Vessby B; Diabetes and Nutrition Study Group (DNSG) of the European Association. Evidence-based nutritional approaches to the treatment and prevention of diabetes mellitus. Nutr Metab Cardiovasc Dis. 2004; 14:373-394.

9. van Bussel BC, Henry RM, Schalkwijk CG, Ferreira I, Feskens EJ, Streppel MT, Smulders YM, Twisk JW, Stehouwer CD. Fish consumption in healthy adults is associated with decreased circulating biomarkers of endothelial dysfunction and inflammation during a 6-year follow-up. J Nutr. 2011; 141:1719-1725.

10. Lopez-Garcia E, Schulze MB, Fung TT, Meigs JB, Rifai N, Manson JE, Hu FB. Major dietary patterns are related to plasma concentrations of markers of inflammation and endothelial dysfunction. Am J Clin Nutr. 2004; 80:1029-1035.

11. Nagel G, Zoller D, Ruf T, Rohrmann S, Linseisen J. Long-term reproducibility of a food-frequency questionnaire and dietary changes in the European Prospective Investigation into Cancer and Nutrition (EPIC)-Heidelberg cohort. Br J Nutr. 2007; 98:194-200.

12. Willett WC. Nutritional Epidemiology. 2th ed. New York, NY: Oxford University Press; 1998.

13. Microvascular and acute complications in IDDM patients: the EURODIAB IDDM Complications Study. Diabetologia 1994; 37:278-285.

14. Chaturvedi N, Bandinelli S, Mangili R, Penno G, Rottiers RE, Fuller JH. Microalbuminuria in type 1 diabetes: rates, risk factors and glycemic threshold. Kidney Int. 2001; 60: 219-227.

15. Chaturvedi N, Fuller JH, Taskinen MR. Differing associations of lipid and lipoprotein disturbances with the macrovascular and microvascular complications of type 1 diabetes. Diabetes Care. 2001; 24: 2071-2077.

16. Schram MT, Chaturvedi N, Schalkwijk C, Giorgino F, Ebeling P, Fuller JH, Stehouwer CD; EURODIAB Prospective Complications Study. Vascular risk factors and markers of endothelial function as determinants of inflammatory markers in type 1 diabetes: the EURODIAB Prospective Complications Study. Diabetes Care. 2003; 26:2165-2173.

17. Toeller M, Klischan A, Heitkamp G, Schumacher W, Milne R, Buyken A, Karamanos B, Gries FA. Nutritional intake of 2868 IDDM patients from 30 centres in Europe. EURODIAB IDDM Complications Study Group. Diabetologia. 1996; 39:929-939.

18. Toeller M, Buyken A, Heitkamp G, Milne R, Klischan A, Gries FA. Repeatability of three-day dietary records in the EURODIAB IDDM Complications Study. Eur J Clin Nutr. 1997; 51:74-80. 
19. Toeller M, Buyken A, Heitkamp G, Brämswig S, Mann J, Milne R, Gries FA, Keen H. Protein intake and urinary albumin excretion rates in the EURODIAB IDDM Complications Study. Diabetologia. 1997; 40:1219-1226.

20. Buyken AE, Toeller M, Heitkamp G, Vitelli F, Stehle P, Scherbaum WA, Fuller JH. Relation of fibre intake to $\mathrm{HbA} 1 \mathrm{C}$ and the prevalence of severe ketoacidosis and severe hypoglycaemia. EURODIAB IDDM Complications Study Group. Diabetologia. 1998; 41:882-890.

21. de Valk-de Roo GW, Stehouwer CD, Lambert J, Schalkwijk CG, van der Mooren MJ, Kluft C, Netelenbos C. Plasma homocysteine is weakly correlated with plasma endothelin and von Willebrand factor but not with endothelium-dependent vasodilatation in healthy postmenopausal women. Clin Chem. 1999; 45: 1200-1205.

22. Chaturvedi N, Stephenson JM, Fuller JH. The relationship between smoking and microvascular complications in the EURODIAB IDDM Complications Study. Diabetes Care. 1995; 18:785-792.

23. Yudkin JS, Stehouwer CD, Emeis JJ, Coppack SW. C-reactive protein in healthy subjects: associations with obesity, insulin resistance, and endothelial dysfunction: a potential role for cytokines originating from adipose tissue? Arterioscler Thromb Vasc Biol. 1999; 19:972-978.

24. de Jager J, Dekker JM, Kooy A, Kostense PJ, Nijpels G, Heine RJ, Bouter LM, Stehouwer CD. Endothelial dysfunction and low-grade inflammation explain much of the excess cardiovascular mortality in individuals with type 2 diabetes: the Hoorn Study. Arterioscler Thromb Vasc Biol. 2006; 26:1086-1093.

25. Twisk JWR, Applied Longitudinal Data Analysis for Epidemiology: A Practical Guide. 1st ed. Cambridge, United Kingdom: Cambridge University; 2003.

26. Ferreira I, Twisk JW, van Mechelen W, Kemper HC, Stehouwer CD. Development of fatness, fitness, and lifestyle from adolescence to the age of 36 years: determinants of the metabolic syndrome in young adults: the amsterdam growth and health longitudinal study. Arch Intern Med 2005; 165: $42-48$.

27. van de Laar RJ, Ferreira I, van Mechelen W, Prins MH, Twisk JW, Stehouwer CD. Lifetime vigorous but not light-to-moderate habitual physical activity impacts favorably on carotid stiffness in young adults: the amsterdam growth and health longitudinal study. Hypertension. 2010; 55:33-39.

28. Eldrup N, Kragelund C, Steffensen R, Nordestgaard BG. Prognosis by C-reactive protein and matrix metalloproteinase-9 levels in stable coronary heart disease during 15 years of follow-up. Nutr Metab Cardiovasc Dis. 2012;22:677-83.

29. Blankenberg S, Rupprecht HJ, Bickel C, Peetz D, Hafner G, Tiret L, Meyer J. Circulating cell adhesion molecules and death in patients with coronary artery disease. Circulation. 2001; 104:1336-1342.

30. Hall WL. Dietary saturated and unsaturated fats as determinants of blood pressure and vascular function. Nutr Res Rev. 2009; 22:18-38. 


\section{Appendix 7}

\section{Statistical analyses}

\section{Overall Z-scores}

The overall Z-scores were calculated as follows: for each individual biomarker, a Z-score was calculated according to the formula: (individual value - population mean) / population standard deviation. The resulting individual biomarker Z-scores were then averaged into an overall Z-score for endothelial dysfunction and an overall Z-score for low-grade inflammation. The endothelial dysfunction overall Z-score consisted of the biomarkers VWf, SVCAM-1 and SE-selectin; and the low-grade inflammation overall Z-score consisted of the biomarkers CRP, IL- 6 and TNF- $\alpha$.

\section{The nutrient residual method}

We used the nutrient residual method to investigate the consumption of fibre (soluble and insoluble), carbohydrate, fat (saturated, monounsaturated and polyunsaturated) cholesterol and protein (animal and vegetable) independent of energy intake. ${ }^{12}$ In short, we calculated energy-adjusted nutrient residuals with the use of linear regression analyses with energy intake as independent variable and the nutrient of interest as dependent variable. ${ }^{12}$ Energy intake may confound any association between nutrient consumption and endothelial dysfunction or low-grade inflammation. First, energy intake may be a primary determinant of endothelial dysfunction or low-grade inflammation. Second, total intake determines the consumption of a specific nutrient unrelated to diet composition because of the positive correlation between energy intake and the consumption of most nutrients. ${ }^{12}$

\section{Additional analyses}

\section{The role of comorbidity}

The results did not materially alter when we additionally adjusted the models for coronary heart disease, cardiovascular disease, retinopathy, neuropathy, albuminuria status, physical activity or anti-hypertensive medication use in 1989 and 1997 or for the individual's educational status (data not shown).

\section{Absolute measures of dietary intake}

When we repeated the analyses by replacing the nutrient residuals for the nutrients in absolute terms, the results showed that the study population consumed per +1 SD of the 1997 endothelial dysfunction Z-score, $0.64 \mathrm{~g} / \mathrm{d}$ less fibre (and divided into its components; $0.23 \mathrm{~g} / \mathrm{d}$ less soluble fibre and $0.44 \mathrm{~g} / \mathrm{d}$ less insoluble fibre); $7 \%$ less polyunsaturated fat (a ratio is mathematically required to express a difference on a 
logarithmic scale); and $0.66 \mathrm{~g} / \mathrm{d}$ less vegetable protein. In addition, the study population consumed per +1 SD of the 1997 low-grade inflammation Z-score, $0.65 \mathrm{~g} / \mathrm{d}$ less fibre; $0.28 \mathrm{~g} / \mathrm{d}$ less soluble fibre; $5 \%$ less polyunsaturated fat; and $4 \%$ more cholesterol (a ratio is mathematically required to express a difference on a logarithmic scale).

\section{Individual biomarkers}

We repeated the analyses with Z-scores of each of the individual biomarkers, as opposed to the composite score, to determine whether nutrient and individual biomarker associations differed within a given family of biomarkers (Figure 7.1). For this purpose, the increments/decrements in nutrient consumption were expressed per +1 SD for each of the 1997 biomarkers. Figure 7.1 shows (A1) that the study population consumed less fibre [both soluble (A2) and insoluble fibre (A3)] per +1 SD for each of the markers, with significant associations for sE-selectin and CRP; (B) that the study population consumed less polyunsaturated fat per +1 SD for each of the markers, except for $\mathrm{VWf}$, with significant associations for SVCAM-1 and TNF- $\alpha$; (C) that the study population consumed more cholesterol per +1 SD for each of the markers, with a significant association for IL-6; (D) that the study population consumed less vegetable protein per +1 SD for each of the markers, except for sE-selectin and TNF- $\alpha$, with significant associations for SVCAM-1 and TNF- $\alpha$.

\section{Handling of missing data}

Of the original 491 individuals in this study, all had dietary data in 1989 and biomarker data in 1997. 324 had additional dietary data in 1997. Full data (diet in 1989 and in 1997, and biomarkers in 1997) were, therefore, available in 324 individuals. However, GEE allowed us to analyze the full dataset of 491 individuals, as it handles missing data robustly. ${ }^{25}$ Nevertheless, when we repeated the analyses on 324 individuals, the results did not materially change. These analyses were however hampered by a reduction in statistical power.

\section{Cross-sectional analyses}

We re-analyzed our data by repeating the statistical analyses with the use of conventional (cross-sectional) linear regression for both sets of dietary data individually (i.e. for the first set of additional analyses: independent variable: 1989 dietary data, dependent variable 1997 biomarker data and, for the second set of additional analyses: independent variable: 1997 dietary data, dependent variable 1997 biomarker data). These results showed similar associations for both sets of dietary data (Table S7.3). 


\section{Changes in nutrient consumption}

We re-analyzed our data and calculated the change in nutrient consumption over time (for each nutrient: energy-adjusted nutrient residual in 1997 minus the energyadjusted nutrient residual in 1989). We then constructed tertiles (for each nutrient individually), which represented: 1 ) those individuals who decreased the consumption of a certain nutrient over time (lowest tertile); 2) those individuals who remained stable with regard to the consumption of certain nutrient over time (middle tertile); and 3) those individuals who increased the consumption of a certain nutrient over time (highest tertile). When we repeated the analyses the results showed that none of the changes in nutrient consumption over time were associated with endothelial dysfunction or low-grade inflammation, except for polyunsaturated fat consumption. The results for polyunsaturated fat consumption showed that individuals who decreased polyunsaturated fat consumption over time had lower endothelial dysfunction and low-grade inflammation scores as compared to those individuals whom remained stable (Table S7.4). The results of these secondary analyses should, however, be interpreted with caution, because a biological explanation is not readily at hand and we cannot exclude the play of chance.

Table S7.1 Additional characteristics of the study population. ${ }^{a}$

\begin{tabular}{|c|c|c|}
\hline Clinical characteristics & baseline 1989 & follow-up 1997 \\
\hline Weight, kg & $67.1 \pm 11.3^{b}$ & $70.1 \pm 12.3$ \\
\hline Waist-to-hip ratio & $0.84 \pm 0.09$ & $0.88 \pm 0.14$ \\
\hline $\mathrm{HbA} 1 \mathrm{c}, \%$ & $8.2 \pm 2.1$ & $8.2 \pm 1.5^{c}$ \\
\hline Frequency of insulin injections, per $d$ & $2.6 \pm 0.8^{d}$ & $2.9 \pm 0.8$ \\
\hline Systolic blood pressure, $\mathrm{mmHg}$ & $121.5 \pm 18.5^{\mathrm{e}}$ & $123.4 \pm 20.1$ \\
\hline Diastolic blood pressure, $\mathrm{mmHg}$ & $76.2 \pm 11.7^{\mathrm{e}}$ & $75.1 \pm 11.3$ \\
\hline Age of last education, years & $19.7 \pm 11.1^{f}$ & $19.7 \pm 11.1^{f}$ \\
\hline Coronary heart disease, $\%$ & $9.0^{\mathrm{g}}$ & $25.5^{\mathrm{g}}$ \\
\hline Cardiovascular disease, $\%$ & $10.1^{b}$ & $27.8^{g}$ \\
\hline Retinopathy none, \% & $54.9^{h}$ & 44.6 \\
\hline Retinopathy background, \% & $32.4^{h}$ & 26.7 \\
\hline Retinopathy proliferative, $\%$ & $12.7^{\mathrm{h}}$ & 28.7 \\
\hline Neuropathy, \% & $34.2^{i}$ & $40.3^{j}$ \\
\hline Albuminuria $<20 \mathrm{mg} / \mathrm{l}, \%$ & $62.7^{k}$ & $62.4^{d}$ \\
\hline Albuminuria 20 - 200 mg/l, \% & $23.9^{k}$ & $14.5^{d}$ \\
\hline Albuminuria > 200 mg/l, \% & $13.3^{k}$ & $23.1^{d}$ \\
\hline \multicolumn{3}{|l|}{ Dietary characteristics } \\
\hline Total fat, g/d & $100 \pm 36$ & $93 \pm 33^{1}$ \\
\hline Saturated fat, g/d & $37 \pm 15$ & $35 \pm 14^{\prime}$ \\
\hline Monounsaturated fat, g/d & $41 \pm 16$ & $38 \pm 15^{\prime}$ \\
\hline Total protein, g/d & $102 \pm 31$ & $97 \pm 30^{1}$ \\
\hline Animal protein, $\mathrm{g} / \mathrm{d}$ & $71 \pm 26$ & $66 \pm 25^{\prime}$ \\
\hline Carbohydrate, g/d & $246 \pm 79$ & $244 \pm 79^{\prime}$ \\
\hline
\end{tabular}

${ }^{\text {a }}$ Data are presented as means \pm standard deviation or percentages as appropriate $(n=491) ;{ }^{b}(n=487)$; $(n=484) ;{ }^{d}(n=489) ;{ }^{e}(n=488) ;{ }^{f}(n=446) ;{ }^{g}(n=490) ;{ }^{h}(n=426) ;{ }^{i}(n=483) ;{ }^{j}(n=464) ;{ }^{k}(n=472){ }^{i}(n=324)$ 
Table S7.2 Nutrient consumption over time per +1 standard deviation of the endothelial dysfunction or low-grade inflammation Z-scores.

\begin{tabular}{lcc}
\hline Nutrients & Endothelial dysfunction $^{\text {a }}$ & Low-grade inflammation \\
\cline { 2 - 3 } Carbohydrate $^{\mathrm{b}}$ & $-0.08(-0.24 ; 0.08)$ & $\beta^{\mathrm{a}}(95 \% \mathrm{Cl})$ \\
Carbohydrate $^{\mathrm{c}}$ & $-0.07(-0.22 ; 0.08)$ & $-0.07(-0.18 ; 0.04)$ \\
Total fat $^{\mathrm{b}}$ & $-0.04(-0.18 ; 0.10)$ & $-0.02(-0.06 ; 0.02)$ \\
Total fat $^{\mathrm{d}}$ & $-0.11(-0.29 ; 0.07)$ & $0.03(-0.08 ; 0.14)$ \\
Saturated fat $^{\mathrm{b}}$ & $-0.02(-0.15 ; 0.11)$ & $-0.04(-0.10 ; 0.02)$ \\
Saturated fat $^{\mathrm{d}}$ & $-0.11(-0.26 ; 0.05)$ & $0.04(-0.07 ; 0.15)$ \\
Monounsaturated fat $^{\mathrm{b}}$ & $0.05(-0.09 ; 0.19)$ & $-0.05(-0.13 ; 0.04)$ \\
Monounsaturated fat $^{\mathrm{d}}$ & $0.03(-0.16 ; 0.22)$ & $0.03(-0.07 ; 0.14)$ \\
Total protein $^{\mathrm{b}}$ & $-0.01(-0.12 ; 0.11)$ & $0.04(-0.05 ; 0.14)$ \\
Total protein $^{\mathrm{e}}$ & $-0.13(-0.31 ; 0.06)$ & $0.02(-0.09 ; 0.13)$ \\
Animal protein $^{\mathrm{b}}$ & $0.05(-0.06 ; 0.15)$ & $-0.04(-0.11 ; 0.04)$ \\
Animal protein $^{\mathrm{e}}$ & $-0.08(-0.23 ; 0.08)$ & $0.06(-0.05 ; 0.17)$ \\
\hline
\end{tabular}

${ }^{\mathrm{a}} \mathrm{B}(95 \% \mathrm{Cl})$ : indicates increments/decrements in nutrient consumption over time per +1 standard deviation of the endothelial dysfunction or low-grade inflammation Z-scores; ${ }^{*} p \leq 0.05$; energy-adjusted nutrient residuals in arbitrary units; $n=491 .{ }^{b}$ Model 1 : crude. ${ }^{c}$ Model 2: adjusted for sex, age, duration of diabetes, investigation centre, BMI, energy intake, smoking behaviour, alcohol, fat, protein, cholesterol and fibre consumption in 1989 and 1997. ' Model 2: adjusted for sex, age, duration of diabetes, investigation centre, BMI, energy intake, smoking behaviour, alcohol, carbohydrate, fibre, cholesterol and protein consumption in 1989 and 1997. ${ }^{~}$ Model 2: adjusted for sex, age, duration of diabetes, investigation centre, BMI, energy intake, smoking behaviour, alcohol, carbohydrate, fibre, cholesterol and fat consumption in 1989 and 1997. 


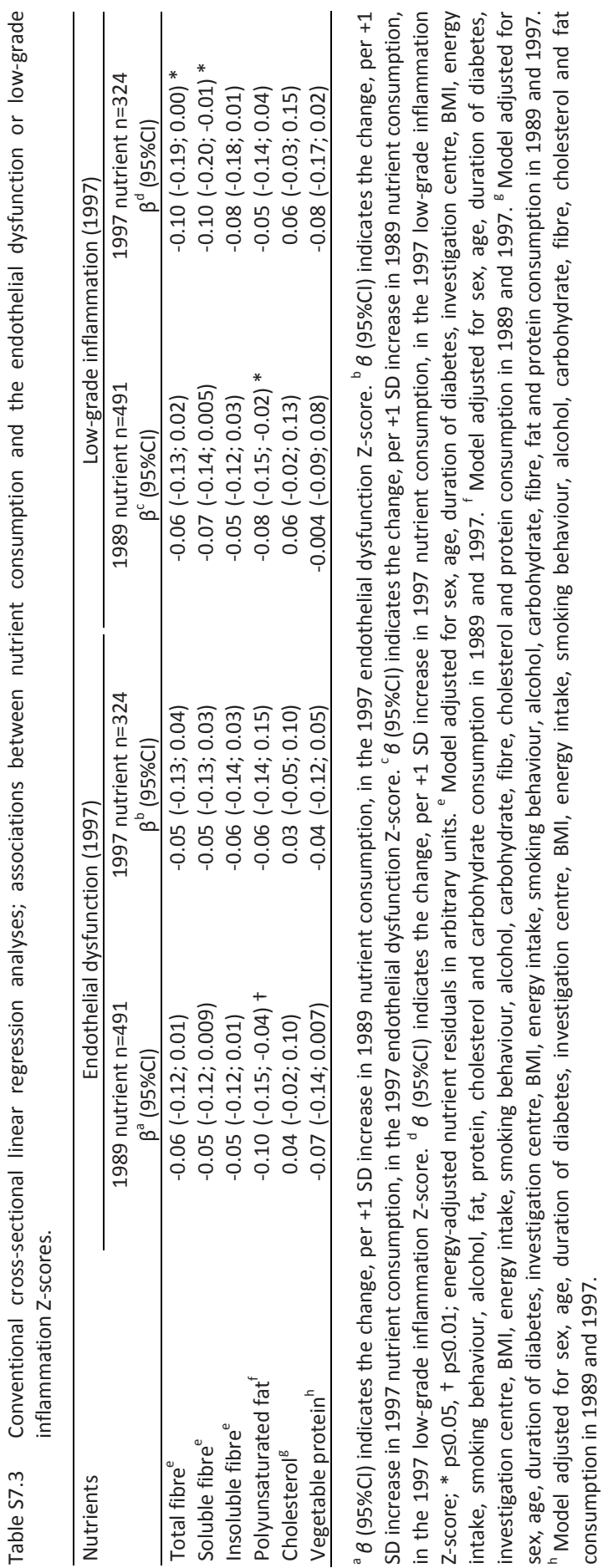


Table S7.4 Conventional linear regression analyses for changes in nutrient consumption over time; associations between changes in nutrient consumption and the endothelial dysfunction or low-grade inflammation Z-scores.

\begin{tabular}{|c|c|c|c|c|}
\hline Nutrients & & $\begin{array}{c}\text { Change nutrient } \\
\text { residual } 1997 \\
\text { minus } 1989 \\
\text { mean } \pm \text { SD }^{\mathrm{a}}\end{array}$ & $\begin{array}{l}\text { Endothelial } \\
\text { dysfunction } \\
\beta^{b}(95 \% \mathrm{Cl})\end{array}$ & $\begin{array}{c}\text { Low-grade } \\
\text { inflammation } \\
\beta^{\mathrm{b}}(95 \% \mathrm{Cl})\end{array}$ \\
\hline \multirow[t]{3}{*}{ Total fibre $^{c}$} & Decreased & $-1.3 \pm 0.8$ & $-0.10(-0.28 ; 0.07)$ & $-0.001(-0.20 ; 0.20)$ \\
\hline & Stable & $-0.02 \pm 0.2$ & - & - \\
\hline & Increased & $1.1 \pm 0.9$ & $-0.01(-0.18 ; 0.16)$ & $0.003(-0.19 ; 0.20)$ \\
\hline \multirow[t]{3}{*}{ Soluble fibre ${ }^{c}$} & Decreased & $-1.3 \pm 0.7$ & $0.02(-0.14 ; 0.19)$ & $0.13(-0.07 ; 0.32)$ \\
\hline & Stable & $0.00 \pm 0.3$ & - & - \\
\hline & Increased & $1.1 \pm 0.9$ & $0.02(-0.15 ; 0.19)$ & $0.05(-0.15 ; 0.25)$ \\
\hline \multirow[t]{3}{*}{ Insoluble fibre ${ }^{c}$} & Decreased & $-1.3 \pm 0.8$ & $-0.06(-0.24 ; 0.11)$ & $-0.06(-0.26 ; 0.15)$ \\
\hline & Stable & $-0.03 \pm 0.2$ & - & - \\
\hline & Increased & $1.1 \pm 0.9$ & $0.01(-0.16 ; 0.18)$ & $-0.02(-0.22 ; 0.17)$ \\
\hline \multirow[t]{3}{*}{ Polyunsaturated fat ${ }^{d}$} & Decreased & $-1.0 \pm 0.6$ & $-0.33(-0.49 ;-0.16)+$ & $-0.25(-0.44 ;-0.05) *$ \\
\hline & Stable & $0.1 \pm 0.2$ & - & - \\
\hline & Increased & $1.1 \pm 0.6$ & $-0.13(-0.29 ; 0.04)$ & $-0.12(-0.32 ; 0.08)$ \\
\hline \multirow[t]{3}{*}{ Cholesterol $^{\mathrm{e}}$} & Decreased & $-1.2 \pm 0.7$ & $0.11(-0.07 ; 0.28)$ & $0.01(-0.18 ; 0.20)$ \\
\hline & Stable & $-0.02 \pm 0.3$ & - & - \\
\hline & Increased & $1.2 \pm 0.7$ & $0.06(-0.11 ; 0.23)$ & $-0.10(-0.29 ; 0.08)$ \\
\hline \multirow[t]{3}{*}{ Vegetable protein $^{f}$} & Decreased & $-1.2 \pm 0.6$ & $-0.04(-0.21 ; 0.13)$ & $0.04(-0.15 ; 0.24)$ \\
\hline & Stable & $-0.01 \pm 0.3$ & - & - \\
\hline & Increased & $1.2 \pm 0.7$ & $-0.02(-0.15 ; 0.12)$ & $-0.08(-0.27 ; 0.12)$ \\
\hline
\end{tabular}

${ }^{\text {a }}$ Data are means \pm standard deviation (SD) that indicate the change in the consumption of a certain nutrient over time; energy-adjusted nutrient residuals in arbitrary units. ${ }^{b} b(95 \% \mathrm{Cl})$ compares individuals who decreased $(n=108)$, who remained stable $(n=108)$ or who increased $(n=108)$ the consumption of a certain nutrient over time ("stable" served as reference category); ${ }^{*} p \leq 0.05,+p \leq 0.01 ; n=324$. 'Model adjusted for sex, age, duration of diabetes, investigation centre, BMI, energy intake, smoking behaviour, alcohol, fat, protein, cholesterol and carbohydrate consumption in 1989 and 1997. ' Model adjusted for sex, age, duration of diabetes, investigation centre, BMI, energy intake, smoking behaviour, alcohol, carbohydrate, fibre, cholesterol and protein consumption in 1989 and 1997. ${ }^{\mathrm{e}}$ Model adjusted for sex, age, duration of diabetes, investigation centre, BMI, energy intake, smoking behaviour, alcohol, carbohydrate, fibre, fat and protein consumption in 1989 and $1997 .{ }^{\text {f }}$ Model adjusted for sex, age, duration of diabetes, investigation centre, BMI, energy intake, smoking behaviour, alcohol, carbohydrate, fibre, cholesterol and fat consumption in 1989 and 1997. 
Part III

Endothelial dysfunction, low-grade inflammation and arterial stiffness 


\section{Chapter 8}

\section{Endothelial dysfunction and low-grade inflammation}

are associated with greater arterial stiffness over

a 6-year period

BCT van Bussel*, F Schouten*, RMA Henry, CG Schalkwijk, MR de Boer, I Ferreira, YM Smulders, JWR Twisk, CDA Stehouwer

* both authors contributed equally

Hypertension 2011;58:588-595

Reprinted with permission of the publisher 


\begin{abstract}
Endothelial dysfunction and low-grade inflammation are associated with cardiovascular disease. Arterial stiffening plays an important role in cardiovascular disease and thus may be a mechanism through which endothelial dysfunction and/or low-grade inflammation lead to cardiovascular disease. We investigated the associations between, on the one hand, biomarkers of endothelial dysfunction (soluble endothelial selectin, thrombomodulin and both vascularand intercellular adhesion molecules 1 and von Willebrand factor) and of low-grade inflammation (C-reactive protein, serum amyloid A, interleukin 6 , interleukin 8 , tumour necrosis factor $\alpha$ and soluble intercellular adhesion molecule 1 ) and, on the other hand, arterial stiffness over a 6-year period, in 293 healthy adults ( 155 women). Biomarkers were combined into mean Z-scores. Carotid, femoral and brachial arterial stiffness and carotid-femoral pulse wave velocity were determined by ultrasonography. Measurements were obtained when individuals were 36 and 42 years of age. Associations were analyzed with generalized estimating equations and adjusted for sex, height and mean arterial pressure. The endothelial dysfunction Z-score was inversely associated with femoral distensibility $[\beta(95 \% \mathrm{Cl})-0.51(-0.95 ;-0.06)]$ and compliance coefficients [-0.041 (-0.076;-0.006)], but not with carotid or brachial stiffness or carotid-femoral pulse wave velocity. The low-grade inflammation Z-score was inversely associated with femoral distensibility $[-0.51(-0.95 ;-0.07)]$ and compliance coefficients $[-0.050(-0.084 ;-0.016)]$, and with carotid distensibility coefficient $[-0.91(-1.81 ;-0.008)]$, but not with brachial stiffness or carotidfemoral pulse wave velocity. Biomarkers of endothelial dysfunction and low-grade inflammation are associated with greater arterial stiffness. This provides evidence that arterial stiffening may be a mechanism through which endothelial dysfunction and low-grade inflammation lead to cardiovascular disease.
\end{abstract}




\section{Introduction}

From observational studies ${ }^{1-5}$, it has become increasingly clear that biomarkers of endothelial dysfunction and low-grade inflammation are closely associated with (incident) cardiovascular disease (CVD). In part, these associations can be explained by the fact that endothelial dysfunction and low-grade inflammation play key roles in atherothrombosis. ${ }^{6}$ However, other mechanisms may also be important. In this respect, arterial stiffening may constitute one such mechanism. First, greater arterial stiffness has been shown to be independently associated with cardiovascular morbidity and mortality. ${ }^{7,8}$ Second, endothelial dysfunction and low-grade inflammation affect the composition of the subendothelial matrix, which is an important determinant of arterial stiffness. ${ }^{9}$

Indeed, the association between biomarkers of endothelial dysfunction and low-grade inflammation, and greater arterial stiffness is receiving increasing attention. ${ }^{10,11}$ However, previous studies on this topic were cross-sectional ${ }^{10-26}$ and most focused on either biomarkers of endothelial dysfunction ${ }^{24}$ or low-grade inflammation ${ }^{12,16-23,25,26}$, and were done in middle-aged or elderly populations. ${ }^{10,11,13-15,18-23,26}$ Importantly, endothelial dysfunction and low-grade inflammation are closely linked and difficult to separate conceptually ${ }^{27}$, and any relationship with arterial stiffening may therefore be interdependent. In addition, the process of arterial stiffening is known to start at an early age. ${ }^{28}$

In view of the above, we hypothesize that the development of biomarkers of endothelial dysfunction and low-grade inflammation is associated with arterial stiffening in early adulthood. Therefore, we have measured biomarkers of endothelial dysfunction, low-grade inflammation and arterial stiffness twice in a population-based cohort, i.e. for the first time at the age of 36 years and for the second time at the age of 42 years. We have investigated the 6-year longitudinal associations between biomarkers of endothelial dysfunction and low-grade inflammation, on the one hand, and stiffness estimates of the carotid, femoral and brachial arteries and of the carotidfemoral segment, on the other, in apparently healthy adults of the Amsterdam Growth and Health Longitudinal Study (AGAHLS).

\section{Methods}

\section{Study population}

Data were derived from the AGAHLS, an observational, longitudinal study that started in 1976 with a group of 698 boys and girls (details described elsewhere). ${ }^{29}$ Briefly, its initial goal was to study the natural development of the growth, health and lifestyle of adolescents and to investigate longitudinal relationships between biological and lifestyle variables. The mean age of the individuals at the start of the study was $13.1 \pm 0.8$ [mean \pm standard deviation $(S D)$ ] years. Since then, extensive follow-up 
measurements have been done and the cohort is still under investigation. At each follow-up measurement, anthropometric (body height, weight and skinfolds), biological (blood pressure, serum lipoprotein levels and physical fitness), lifestyle (nutritional habits, smoking behaviour and daily physical activity) and psychological variables were assessed. ${ }^{29}$

In addition, in blood samples of the individuals attending the 2000 and 2006 follow-up examinations (one batch), five biomarkers of endothelial dysfunction and six biomarkers of low-grade inflammation were measured and at both examinations arterial properties were assessed by ultrasonography. At the first ultrasound examination, 377 individuals participated and complete data on stiffness estimates of the carotid, femoral and brachial arteries were obtained in 373 individuals. From these, complete data on all three arteries were obtained in 293 individuals during the follow-up ultrasound examination, all of whom had full data on biomarkers of endothelial dysfunction and low-grade inflammation. The present study was therefore conducted with these 293 individuals.

The study was approved by the local ethics committee of the Vrije Universiteit University Medical Centre and all participants gave their written informed consent.

\section{Assessment of endothelial dysfunction and low-grade inflammation}

Serum biomarkers of endothelial dysfunction [soluble intercellular adhesion molecule 1 (sICAM-1), soluble vascular cell adhesion molecule 1 (sVCAM-1), soluble endothelial selectin (sE-selectin) and soluble thrombomodulin (sTM)], and of low-grade inflammation [C-reactive protein (CRP), serum amyloid A (SAA), interleukin 6 (IL-6), interleukin 8 (IL-8), tumour necrosis factor $\alpha$ (TNF- $\alpha$ ) and sICAM-1] were assessed by an electrochemiluminescence detection system using multi-array technology (SECTOR Imager 2400, Meso Scale Discovery, USA) as described elsewhere ${ }^{30}$ (please see Appendix 8 for details).

In addition, a plasma biomarker of endothelial dysfunction [von Willebrand factor (vWf)] was determined in citrated plasma by means of ELISA as described elsewhere ${ }^{30}$ (please see Appendix 8 for details).

\section{Arterial stiffness}

We assessed local arterial stiffness of the carotid, femoral and brachial arteries and central arterial stiffness [the carotid-femoral pulse wave velocity (cfPWV)] ${ }^{31,32}$ (please see Appendix 8 for details).

\section{Other measurements}

Weight, height, body mass index, heart rate and blood pressure were determined according to international standards and smoking behaviour was assessed by questionnaire as previously described. ${ }^{29,31}$ Total and high-density lipoprotein 
cholesterol, triglycerides and glycated haemoglobin were determined as previously described. ${ }^{29,31}$ Hypertension was defined as a systolic blood pressure $>140 \mathrm{mmHg}$ and/or a diastolic blood pressure $>90 \mathrm{mmHg}$, and/or treatment for hypertension.

\section{Statistical analyses}

All analyses were performed with SPSS (Statistical Package for Social Sciences, version 15, USA). Variables with a skewed distribution (CRP, SAA, IL-6 and triglycerides) were transformed using a natural logarithm (In). Overall Z-scores were calculated for endothelial dysfunction or low-grade inflammation (please see Appendix 8). We used generalized estimating equations (GEE) to examine the associations between either the overall Z-score for endothelial dysfunction or the overall Z-score for low-grade inflammation and arterial stiffness over the 6-year study period. In these analyses an exchangeable correlation structure was used. GEE analysis is a method for longitudinal data analyses and takes into account the correlation of repeated measurements within individuals over time. ${ }^{33}$

First, we investigated the associations between the overall Z-scores for either endothelial dysfunction or low-grade inflammation on the one hand and arterial stiffness estimates on the other with adjustments for sex, height, and mean arterial pressure.

Second, the analyses were repeated with mutual adjustments for the overall Z-scores for either low-grade inflammation or endothelial dysfunction. Third, to gain further insight into which of the individual elements of the stiffness formulas might have been primarily responsible for any relationships between endothelial dysfunction or lowgrade inflammation and arterial stiffness estimates, the analyses were repeated with the individual elements of the stiffness formulas (diameter, distension, pulse pressure and intima-media thickness) as dependent variables.

Data are presented as mean $( \pm$ SD) or median (interquartile range) for skewed variables and regression coefficients $(\beta)$ with their $95 \%$ confidence intervals $(95 \% \mathrm{Cl})$. A two-sided $p$-value $<0.05$ was considered statistically significant.

\section{Results}

Table 8.1 shows the general characteristics, the biomarker concentrations and the stiffness estimates of the study population. Systolic blood pressure remained stable (116 $\mathrm{mmHg})$, diastolic blood pressure increased $(+5.5 \mathrm{mmHg})$, pulse pressure decreased $(-5.6 \mathrm{mmHg})$ and heart rate decreased $(-8.9 \mathrm{bpm})$ during the 6-year followup. Biomarkers of low-grade inflammation remained fairly stable over 6-year followup, except for a decrease in TNF- $\alpha$. Biomarkers of endothelial dysfunction remained stable or increased over 6-year follow-up, except for a decrease in sE-selectin. Pulse pressure and femoral and brachial artery stiffness decreased during 6-year follow-up. Nevertheless, carotid artery stiffness increased [for distensibility coefficient (DC) 
$-1.0010^{-3} / \mathrm{kPA}$ and for Young's elastic modulus (YEM) $+0.025 * 10^{3} / \mathrm{kPa}$ ] during the 6-year follow-up.

Table 8.1 Characteristics of the study population.

\begin{tabular}{|c|c|c|c|}
\hline & 2000 & 2006 & p-value \\
\hline Age, years & $36.6 \pm 0.6$ & $42.6 \pm 0.6$ & $<0.001$ \\
\hline Women, \% & 52.9 & 52.9 & - \\
\hline Smoker, \% & $64,21.9$ & $45,15.4$ & $<0.001$ \\
\hline Systolic blood pressure, $\mathrm{mmHg}$ & $116.3 \pm 11.7$ & $116.2 \pm 14.4$ & 0.858 \\
\hline Diastolic blood pressure, $\mathrm{mmHg}$ & $64.9 \pm 7.2$ & $70.4 \pm 8.1$ & $<0.001$ \\
\hline Pulse pressure, $\mathrm{mmHg}$ & $51.4 \pm 6.5$ & $45.8 \pm 9.0$ & $<0.001$ \\
\hline Mean arterial pressure, $\mathrm{mmHg}$ & $82.1 \pm 8.7$ & $85.1 \pm 9.9$ & $<0.001$ \\
\hline Prevalence of hypertension, \% & 5.1 & 9.6 & 0.015 \\
\hline Heart rate, bpm & $70.5 \pm 11.4$ & $61.6 \pm 9.0$ & $<0.001$ \\
\hline Body height, $\mathrm{cm}$ & $177.0 \pm 9.2$ & $177.4 \pm 9.0$ & $<0.001$ \\
\hline Body weight, kg & $75.8 \pm 12.7$ & $77.9 \pm 13.7$ & $<0.001$ \\
\hline Body mass index, $\mathrm{kg} / \mathrm{m}^{2}$ & $24.1 \pm 3.2$ & $24.7 \pm 3.6$ & $<0.001$ \\
\hline Total cholesterol, mmol/l & $5.0 \pm 0.9$ & $5.0 \pm 0.8$ & 0.315 \\
\hline High density lipoprotein cholesterol, mmol/l & $1.4 \pm 0.4$ & $1.7 \pm 0.4$ & $<0.001$ \\
\hline Triglycerides, $\mathrm{mmol} / \mathrm{l}$ & $1.0(0.8-1.5)$ & $1.0(0.7-1.4)$ & 0.018 \\
\hline $\mathrm{HbA1c}, \%$ & $5.2 \pm 0.4$ & $5.4 \pm 0.4$ & $<0.001$ \\
\hline \multicolumn{4}{|l|}{ Biomarkers } \\
\hline C-reactive protein, $\mathrm{mg} / \mathrm{l}$ & $0.8(0.3-2.0)$ & $0.8(0.3-1.8)$ & 0.946 \\
\hline Serum amyloid $A, \mathrm{mg} / \mathrm{l}$ & $1.2(0.7-2.2)$ & $1.3(0.7-2.3)$ & 0.099 \\
\hline Interleukin 6, ng/l & $2.3(1.7-3.7)$ & $2.4(1.8-3.7)$ & 0.664 \\
\hline Interleukin 8, ng/l & $9.2 \pm 3.4$ & $9.5 \pm 4.1$ & 0.190 \\
\hline Tumour necrosis factor $\alpha, \mathrm{ng} / \mathrm{l}$ & $9.3 \pm 3.4$ & $9.0 \pm 3.3$ & 0.006 \\
\hline Soluble intercellular adhesion molecule $1, \mu \mathrm{g} / \mathrm{l}$ & $203.3 \pm 52.4$ & $200.3 \pm 41.2$ & 0.142 \\
\hline Soluble vascular cellular adhesion molecule $1, \mu \mathrm{g} / \mathrm{l}$ & $332.8 \pm 81.2$ & $333.9 \pm 69.2$ & 0.767 \\
\hline Soluble endothelial selectin, $\mu \mathrm{g} / \mathrm{l}$ & $10.5 \pm 4.7$ & $10.1 \pm 4.6$ & 0.010 \\
\hline Soluble thrombomodulin, $\mu \mathrm{g} / \mathrm{l}$ & $2.47 \pm 0.66$ & $2.53 \pm 0.62$ & 0.023 \\
\hline von Willebrand factor, \% & $102.5 \pm 40.0$ & $112.8 \pm 42.4$ & $<0.001$ \\
\hline \multicolumn{4}{|l|}{ Carotid artery } \\
\hline Distensibility coefficient, $10^{-3} / \mathrm{kPa}$ & $26.7 \pm 6.1$ & $25.7 \pm 7.2$ & 0.014 \\
\hline Compliance coefficient, $\mathrm{mm}^{2} / \mathrm{kPa}$ & $1.0 \pm 0.3$ & $1.0 \pm 0.3$ & 0.143 \\
\hline Young's elastic modulus, $10^{3} / \mathrm{kPa}$ & $0.44 \pm 0.13$ & $0.47 \pm 0.16$ & 0.007 \\
\hline Diameter, mm & $6.9 \pm 0.6$ & $7.1 \pm 0.7$ & $<0.001$ \\
\hline Distension, mm & $0.6 \pm 0.1$ & $0.5 \pm 0.1$ & $<0.001$ \\
\hline Pulse pressure, $\mathrm{mmHg}$ & $51.3 \pm 7.1$ & $45.9 \pm 9.4$ & $<0.001$ \\
\hline Intima-media thickness, $\mathrm{mm}$ & $0.62 \pm 0.10$ & $0.66 \pm 0.12$ & $<0.001$ \\
\hline \multicolumn{4}{|l|}{ Femoral artery } \\
\hline Distensibility coefficient, $10^{-3} / \mathrm{kPa}$ & $7.1 \pm 3.7$ & $8.3 \pm 4.5$ & $<0.001$ \\
\hline Compliance coefficient, $\mathrm{mm}^{2} / \mathrm{kPa}$ & $0.51 \pm 0.24$ & $0.63 \pm 0.33$ & $<0.001$ \\
\hline Diameter, mm & $9.8 \pm 1.3$ & $10.0 \pm 1.5$ & $<0.001$ \\
\hline Distension, mm & $0.22 \pm 0.10$ & $0.23 \pm 0.10$ & 0.149 \\
\hline Pulse pressure, $\mathrm{mmHg}$ & $51.4 \pm 7.2$ & $46.5 \pm 9.8$ & $<0.001$ \\
\hline \multicolumn{4}{|l|}{ Brachial artery } \\
\hline Distensibility coefficient, $10^{-3} / \mathrm{kPa}$ & $14.6 \pm 9.2$ & $17.6 \pm 11.2$ & $<0.001$ \\
\hline Compliance coefficient, $\mathrm{mm}^{2} / \mathrm{kPa}$ & $0.17 \pm 0.09$ & $0.21 \pm 0.11$ & $<0.001$ \\
\hline Diameter, $\mathrm{mm}$ & $4.0 \pm 0.7$ & $4.0 \pm 0.7$ & 0.187 \\
\hline Distension, $\mathrm{mm}$ & $0.18 \pm 0.09$ & $0.19 \pm 0.10$ & 0.205 \\
\hline Pulse pressure, mmHg & $51.7 \pm 7.2$ & $45.0 \pm 9.5$ & $<0.001$ \\
\hline \multicolumn{4}{|l|}{ Central arterial stiffness } \\
\hline Pulse wave velocity, $\mathrm{m} / \mathrm{s}$ & $7.7 \pm 1.6 *$ & $8.3 \pm 1.6+$ & $<0.001$ \\
\hline
\end{tabular}

Data are reported as mean $\pm S D$, median (interquartile range) or percentage, as appropriate; $p$-value, paired T-test or McNemar's test, as appropriate; $n=293, * n=241,+n=292$ 


\section{Endothelial dysfunction, low-grade inflammation and arterial stiffness}

The endothelial dysfunction overall Z-score was inversely associated with the femoral artery DC $[\beta(95 \% \mathrm{Cl})-0.51(-0.95 ;-0.06), p=0.025]$ and compliance coefficient (CC) $[-0.041(-0.076 ;-0.006), p=0.020]$ after adjustment for sex, height and mean arterial pressure, whereas it was not associated with carotid or brachial artery stiffness (Table 8.2, models 1). Additional adjustment for the low-grade inflammation overall Z-score decreased the regression coefficient $(\beta)$ for the femoral artery DC by $32 \%$ and for the femoral artery CC by $46 \%$ (Table 8.2 , models 2 ). In addition, the endothelial dysfunction overall Z-score was not associated with cfPWV after adjustment for sex, height and mean arterial pressure [0.001 (-0.22;0.22), $p=0.994]$.

The low-grade inflammation overall Z-score was inversely associated with the carotid artery DC [-0.91 (-1.81;-0.008), $\mathrm{p}=0.048]$ and femoral artery DC [-0.51 (-0.95;-0.07), $p=0.024]$ and CC $[-0.050(-0.084 ;-0.016), p=0.004]$ after adjustment for sex, height and mean arterial pressure, whereas it was not associated with carotid artery CC or YEM or brachial artery stiffness (Table 8.2, models 1). Additional adjustment for the endothelial dysfunction overall Z-score decreased the regression coefficient $(\beta)$ for the femoral artery DC by $47 \%$ and for the femoral artery CC by $24 \%$, whereas the result for the carotid artery DC did not materially change (Table 8.2, models 2). In addition, the low-grade inflammation overall Z-score was not associated with cfPWV after adjustment for sex, height and mean arterial pressure [-0.074 $(-0.37 ; 0.24), p=0.626]$.

\section{Endothelial dysfunction, low-grade inflammation and arterial diameter, distension, pulse pressure and intima-media thickness}

The inverse associations between the endothelial dysfunction overall Z-score and the femoral artery DC and CC and the low-grade inflammation overall Z-score and the femoral artery DC and CC were primarily driven through inverse associations with femoral artery distension [for endothelial dysfunction $-0.013(-0.025 ;-0.001), p=0.029$; for low-grade inflammation $-0.015(-0.027 ;-0.004), p=0.010$; please see Appendix 8, Table S8.1]. In contrast, the inverse association between the low-grade inflammation overall Z-score and the carotid artery DC was not primarily driven through any of the elements of the stiffness formulas (please see Appendix 8, Table S8.1).

\section{Additional analyses}

Additional adjustment for age (the age range by design being extremely narrow), smoking behaviour, total and HDL cholesterol, and hypertension did not materially change the associations, whereas adjustment for weight did attenuate the regression coefficients with $\sim 50 \%$ (please see Appendix 8, Table S8.2). None of the individuals had diabetes mellitus. 


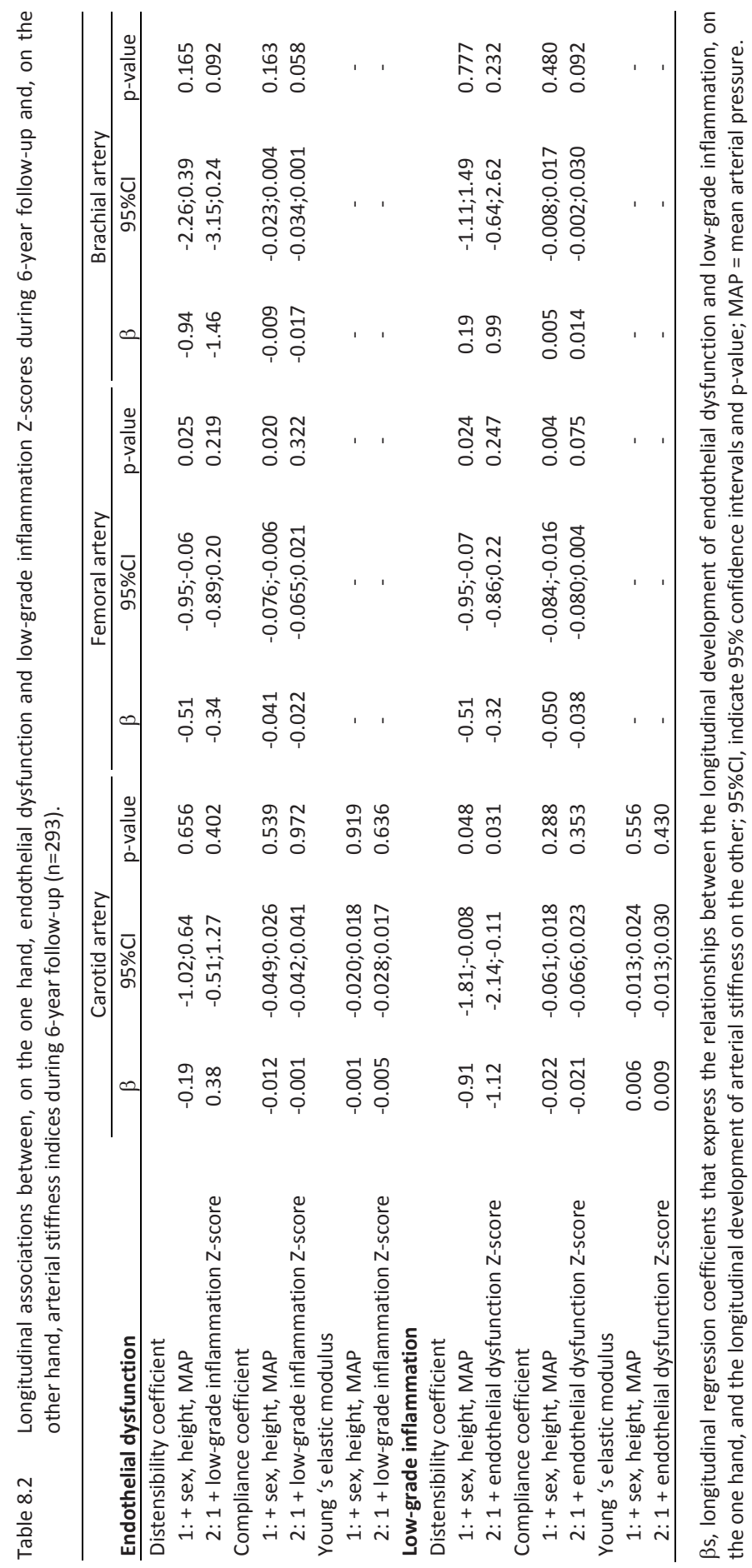


In the analyses for the individual elements of the low-grade inflammation Z-score, the results showed that InSAA and InCRP were the strongest determinants of the carotid artery DC (Figure 8.1A), whereas SICAM-1 and InIL-6 were the strongest determinants of the femoral artery DC (Figure 8.1B), and SICAM-1 and InCRP were the strongest determinants for the femoral artery CC (Figure 8.1C). For the endothelial dysfunction Z-score, sICAM-1 and SE-selectin were the strongest determinants for the femoral artery DC (Figure 8.1B) and CC (Figure 8.1C).

To investigate whether the longitudinal associations (by GEE) were primarily determined by the between- or the within-subject associations over the 6-year study period, we calculated changes (i.e. within-subject) in the endothelial dysfunction overall Z-score, the low-grade inflammation overall Z-score and the arterial stiffness estimates. Then, we re-analyzed the data with the use of linear regression analyses. Changes in the endothelial dysfunction overall Z-score or in the low-grade inflammation overall Z-score were not associated with changes in arterial stiffness estimates (data not shown). This suggests that the reported associations were primarily determined by the between-subject associations over the 6-year study period.

\section{Discussion}

The present investigation is the first to prospectively evaluate, in apparently healthy adults, the relationship between the development of an extensive array of biomarkers of endothelial dysfunction and low-grade inflammation on the one hand and arterial stiffness on the other. The study had three main findings. First, biomarker scores for endothelial dysfunction and low-grade inflammation were associated with greater arterial stiffness over a 6 -year period. The biomarker score for endothelial dysfunction was associated with greater femoral artery stiffness, whereas the biomarker score for low-grade inflammation was associated with both greater carotid and femoral artery stiffness. However, both the biomarker scores for endothelial dysfunction and lowgrade inflammation were not associated with stiffness of the carotid-femoral segment. Endothelial dysfunction and/or low-grade inflammation may thus affect arterial stiffening in a way that depends upon the arterial territory under study. ${ }^{34}$ Second, mutual adjustment for either the biomarker score for low-grade inflammation or endothelial dysfunction showed that the associations between either the biomarker score for endothelial dysfunction or low-grade inflammation with femoral artery stiffness were interdependent. Finally, the associations between each of the biomarker scores and greater femoral artery stiffness were primarily driven through decreased distension, whereas the association between low-grade inflammation and greater carotid stiffness was not primarily driven through any of the arterial parameters. 
A

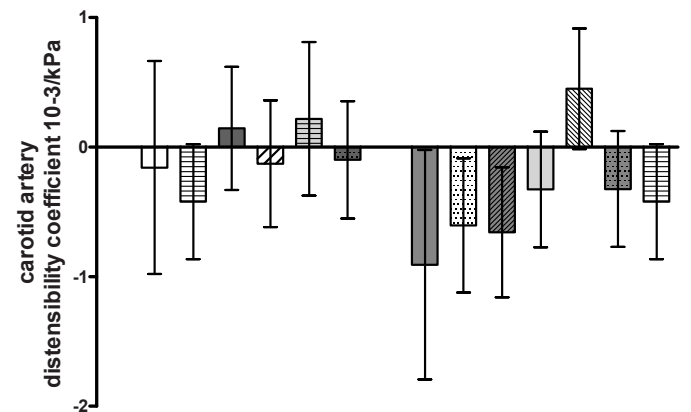

B

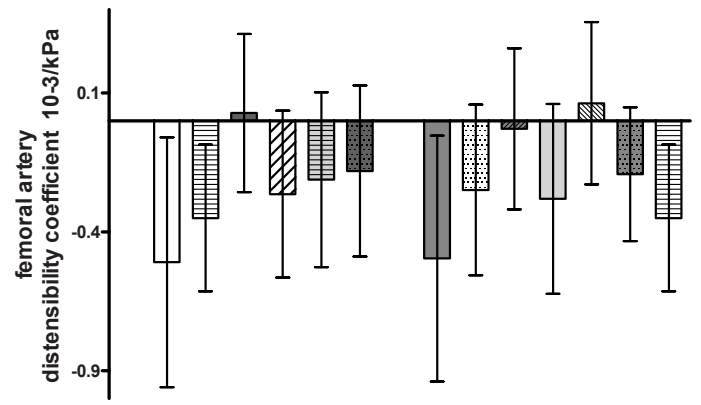

c

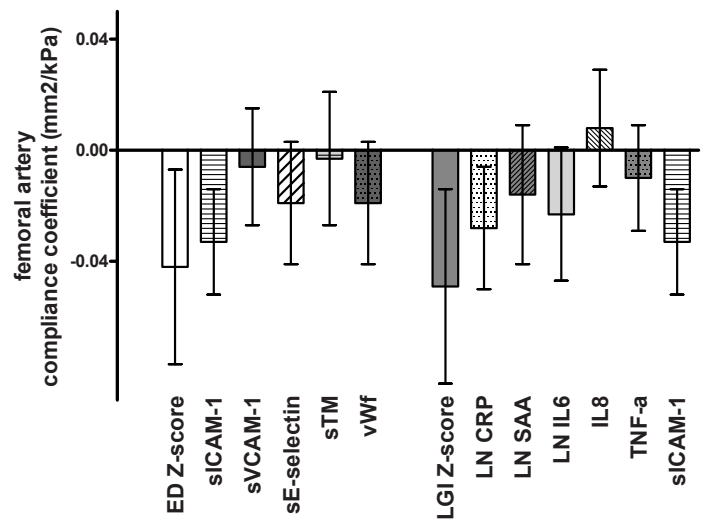

Figure 8.1 Bars are regression coefficients that indicate the associations between either the endothelial dysfunction (ED) Z-score, or the low-grade inflammation (LGI) Z-score or each of the individual biomarker Z-scores and the carotid distensibility coefficient $(A)$, the femoral distensibility coefficient (B) and the femoral compliance coefficient (C) over the 6-year study period. Whiskers indicate the $95 \%$ confidence intervals. All results are adjusted for sex, height and mean arterial pressure. sICAM 1 = soluble intercellular adhesion molecule 1 ; sVCAM-1 = soluble vascular cell adhesion molecule $1 ;$ sE-selectin = soluble endothelial selectin; sTM = soluble thrombomodulin; $\mathrm{vWf}=$ von Willebrand factor; $\mathrm{CRP}=\mathrm{C}$-reactive protein; $\mathrm{SAA}=$ serum amyloid A; IL-6 = interleukin 6; IL-8 = interleukin 8; TNF- $\alpha=$ tumour necrosis factor $\alpha$; LN = log normalized. 
The endothelium has many functions and is itself heterogeneous. ${ }^{35,36}$ The concept of endothelial dysfunction therefore has many dimensions. ${ }^{37} \mathrm{VWf}$, sVCAM-1, sE-selectin,

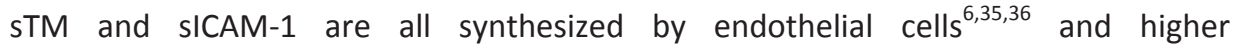
concentrations of these biomarkers are associated with (incident) cardiovascular disease..$^{1-5}$ Consequently, it is plausible to assume that higher circulating concentrations of these biomarkers reflect greater dysfunction.

A limitation of this study is that a measure of NO-mediated dilation, which represents an important function of the endothelium ${ }^{35-37}$, was not available. Nevertheless, both biomarkers of the endothelium and NO-mediated dilation (such as flow-mediated dilation) have been associated with (incident) cardiovascular disease. ${ }^{1-5,38}$

Furthermore, endothelial dysfunction is closely linked with low-grade inflammation and therefore these concepts are difficult to separate. ${ }^{27} \mathrm{We}$, indeed, show that the associations with femoral artery stiffening are dependent on both endothelial dysfunction and low-grade inflammation, whereas this was not the case for carotid artery stiffening. This suggests that endothelial dysfunction and low-grade inflammation might affect arterial stiffening in concert or independently, dependent on the arterial territory under study.

The present investigation was comprehensive and had advantages over previous ones, which investigated either biomarkers of endothelial dysfunction ${ }^{24}$ or low-grade inflammation ${ }^{12,16-23,25,26}$, measured a less extensive array of biomarkers of endothelial dysfunction and low-grade inflammation ${ }^{10-26}$, concerned middle-aged to elderly individuals $^{10,11,13-15,18-23,26}$, targeted one type of artery only ${ }^{10-25}$ and were crosssectional by design. ${ }^{10-26}$

On aggregate, previous studies have shown higher CRP levels to be associated with greater arterial stiffness of elastic ${ }^{10-14,16-23}$, muscular ${ }^{25}$ or both types of arteries ${ }^{26}$, and higher IL-6 levels to be associated with greater elastic arterial stiffness ${ }^{11,15}$. Taken together, these studies ${ }^{10-23,25,26}$ and the present results support the concept that lowgrade inflammation may affect arterial stiffness. In particular, we show that this process is present even in young, apparently healthy adults.

With regard to biomarkers of endothelial dysfunction, previous studies have shown heterogeneous results for $\mathrm{VWf}$ and SICAM-1, some reporting positive associations with elastic arterial stiffness ${ }^{15,24}$, whereas others did not. ${ }^{11,13,14}$ We show that the overall endothelial dysfunction Z-score was significantly associated with femoral artery stiffness (Figure 8.1B and 8.1C), whereas none of the individual endothelial dysfunction biomarkers was, except for sICAM-1. This may be explained by the fact that in these young, apparently healthy individuals, endothelial dysfunction may not be very far advanced and therefore only the sum of endothelial dysfunction biomarkers reveals its association with greater arterial stiffness.

Endothelial dysfunction may influence arterial stiffening by affecting vascular smooth muscle cell tone due to the reduced availability of nitric oxide and the increased activity of vasoconstrictor substances such as endothelin $-1^{9}$, and by affecting the composition of the extracellular matrix. ${ }^{9}$ In addition, inflammation may induce both 
functional and structural changes in the arterial wall via the increased production of reactive oxygen species ${ }^{39}$, which in turn triggers an inflammatory process leading to the proliferation of smooth muscle cells, the influx of leucocytes and the production of pro-inflammatory substances and chemo-attractants. ${ }^{6}$ Our data support the notion that both endothelial dysfunction and low-grade inflammation may cause increased muscular artery stiffness via similar pathophysiological pathways as in the femoral artery both endothelial dysfunction and low-grade inflammation primarily appeared to affect arterial distension (please see Appendix 8, Table S8.1). In contrast, only lowgrade inflammation affected (elastic) carotid artery stiffness, most likely through a pathophysiological mechanism independent of endothelial dysfunction, and not specifically driven through diameter, distension, pulse pressure or intima-media thickness. This suggests that low-grade inflammation may cause increased arterial stiffness of elastic arteries by affecting multiple elements of the vascular wall. These observations particularly align with the notion that the athero- and arteriosclerotic processes are, at least in part, inflammatory in origin and that inflammatory changes may lead to an altered matrix homeostasis (affecting vascular smooth muscle cells and matrix proteins), which in turn leads to a decrease in (femoral) distension. Taken together, the above suggests that endothelial dysfunction and low-grade inflammation may affect arterial stiffness of either muscular or elastic arteries differently. This might explain why endothelial dysfunction and low-grade inflammation were not associated with cfPWV, as the carotid-femoral segment includes both elastic and muscular arterial components. Alternatively, the adults investigated were young and did not have central arterial stiffening, which typically occurs after the age of 60 years. ${ }^{7}$

Additional adjustment for potential confounders did not materially change the results, whereas adjustment for body weight attenuated the associations between biomarkers of endothelial dysfunction or low-grade inflammation and arterial stiffness. However, it is questionable whether body weight should be seen as a true confounder in this relationship. It is biologically more plausible that body weight is part of the causal pathway, i.e. body weight influences biomarkers of endothelial dysfunction and lowgrade inflammation ${ }^{40}$ and these influence arterial stiffening. ${ }^{9}$

Finally, the timeframe in which endothelial dysfunction or low-grade inflammation have their possible impacts on arterial stiffness is unknown and might differ from the 6-year period of the present investigation. (This might explain why changes in biomarkers of endothelial dysfunction or low-grade inflammation did not parallel changes in arterial stiffness.) The question remains for what period a person should have endothelial dysfunction or low-grade inflammation before it affects the vasculature. In any case, our results may suggest that any inflammatory changes that have led to arterial stiffening have already occurred before the age of 36 years.

The present investigation had some limitations. First, intrinsic to an overall Z-score is the assumption that each biomarker in the Z-score carries similar weight. This might have caused us to underestimate the reported associations, due to error because of a 
mathematical approach that might not optimally reflect pathophysiology. Second, the increase in diastolic blood pressure of $6 \mathrm{mmHg}$ and the practically unchanged levels of systolic blood pressure between the ages of 36 and 42 in this study population led to a decrease in pulse pressure. Although this may seem 'unexpected', increases in pulse pressure with ageing are often observed after the $5^{\text {th }}$ or $6^{\text {th }}$ decades of life as a consequence of arterial stiffening. ${ }^{7}$ In addition, these changes were in line with previous life course trends in changes of the two blood pressure components in this cohort, showing steeper increases in (sitting) diastolic $(0.6 \mathrm{mmHg} / \mathrm{year})$ than systolic blood pressure $(0.2 \mathrm{mmHg} /$ year) between late adolescence (age 15) and age 36 (data not shown). Still, as different blood pressure devices were used during the two measurement periods, it is possible that 2000's diastolic blood pressure data may have been underestimated as compared to the 2006 data. As a consequence, at the population level, pulse pressure and all arterial stiffness estimates are most likely underestimated in 2006 as compared to 2000. Nevertheless, a systematic underestimation of stiffness does not materially change the reported associations. Third, on the population level, heart rate was higher in 2000 as compared to 2006. This might be explained by the fact that the ultrasound examination was introduced for the first time in 2000, and, at the second ultrasound examination, individuals might have been more relaxed as they knew what was going to happen. As a (patho)physiological explanation is not readily at hand, such an effect might explain the difference in heart rate. Again, however, this would not be expected to affect the associations observed. Fourth, renal function was only determined in 2006. Although additional adjustment did not materially change our results, the present study, therefore, can only partly exclude a role for renal function in the reported associations. Finally, our data were obtained in a young, Caucasian population and therefore inferences to older individuals and/or other ethnicities should be made with caution.

\section{Perspectives}

Both scores of biomarkers for endothelial dysfunction and low-grade inflammation are associated with greater arterial stiffness in apparently healthy adults, over a 6 -year period. This suggests that arterial stiffness may be a mechanism through which endothelial dysfunction and low-grade inflammation lead to cardiovascular disease. In addition, the results showed that early in the development of arterial stiffening, endothelial dysfunction and low-grade inflammation act differentially along the arterial tree. These data may help to understand the pathophysiology of early arterial stiffening and may provide potential targets for intervention. 


\section{References}

1. Blankenberg S, Rupprecht HJ, Bickel C, Peetz D, Hafner G, Tiret L, Meyer J. Circulating cell adhesion molecules and death in patients with coronary artery disease. Circulation. 2001; 104:1336-1342.

2. de Jager J, Dekker JM, Kooy A, Kostense PJ, Nijpels G, Heine RJ, Bouter LM, Stehouwer CD. Endothelial dysfunction and low-grade inflammation explain much of the excess cardiovascular mortality in individuals with type 2 diabetes: the Hoorn Study. Arterioscler Thromb Vasc Biol. 2006; 26:1086-1093.

3. Jager A, van Hinsbergh VW, Kostense PJ, Emeis JJ, Yudkin JS, Nijpels G, Dekker JM, Heine RJ, Bouter LM, Stehouwer CD. von Willebrand factor, C-reactive protein, and 5-year mortality in diabetic and nondiabetic subjects: the Hoorn Study. Arterioscler Thromb Vasc Biol. 1999; 19:3071-3078.

4. Morange PE, Simon C, Alessi MC, Luc G, Arveiler D, Ferrieres J, Amouyel P, Evans A, Ducimetiere P, Juhan-Vague I. Endothelial cell markers and the risk of coronary heart disease: the Prospective Epidemiological Study of Myocardial Infarction (PRIME) study. Circulation. 2004; 109:1343-1348.

5. Ridker PM, Hennekens $\mathrm{CH}$, Buring JE, Rifai N. C-reactive protein and other markers of inflammation in the prediction of cardiovascular disease in women. N Engl J Med. 2000; 342:836-843.

6. Ross R. Atherosclerosis--an inflammatory disease. N Engl J Med. 1999; 340:115-126.

7. Franklin SS, Khan SA, Wong ND, Larson MG, Levy D. Is pulse pressure useful in predicting risk for coronary heart Disease? The Framingham heart study. Circulation. 1999; 100:354-360.

8. Laurent S, Boutouyrie P, Asmar R, Gautier I, Laloux B, Guize L, Ducimetiere P, Benetos A. Aortic stiffness is an independent predictor of all-cause and cardiovascular mortality in hypertensive patients. Hypertension. 2001; 37:1236-1241.

9. Stehouwer CD, Henry RM, Ferreira I. Arterial stiffness in diabetes and the metabolic syndrome: a pathway to cardiovascular disease. Diabetologia. 2008; 51:527-539.

10. Lieb W, Larson MG, Benjamin EJ, Yin X, Tofler GH, Selhub J, Jacques PF, Wang TJ, Vita JA, Levy D, Vasan RS, Mitchell GF. Multimarker approach to evaluate correlates of vascular stiffness: the Framingham Heart Study. Circulation. 2009; 119:37-43.

11. Schnabel R, Larson MG, Dupuis J, Lunetta KL, Lipinska I, Meigs JB, Yin X, Rong J, Vita JA, Newton-Cheh C, Levy D, Keaney JF, Jr., Vasan RS, Mitchell GF, Benjamin EJ. Relations of inflammatory biomarkers and common genetic variants with arterial stiffness and wave reflection. Hypertension. 2008; 51:1651-1657.

12. Abramson JL, Weintraub WS, Vaccarino V. Association between pulse pressure and C-reactive protein among apparently healthy US adults. Hypertension. 2002; 39:197-202.

13. Amar J, Ruidavets JB, Bal Dit Sollier C, Bongard V, Boccalon H, Chamontin B, Drouet L, Ferrieres J. Soluble CD14 and aortic stiffness in a population-based study. J Hypertens. 2003; 21:1869-1877.

14. Amar J, Ruidavets JB, Sollier CB, Bongard V, Boccalon H, Chamontin B, Drouet L, Ferrieres J. Relationship between $C$ reactive protein and pulse pressure is not mediated by atherosclerosis or aortic stiffness. J Hypertens. 2004; 22:349-355.

15. Chae CU, Lee RT, Rifai N, Ridker PM. Blood pressure and inflammation in apparently healthy men. Hypertension. 2001; 38:399-403.

16. Eklund C, Kivimaki M, Islam MS, Juonala M, Kahonen M, Marniemi J, Lehtimaki T, Viikari J, Raitakari OT, Hurme M. C-reactive protein genetics is associated with carotid artery compliance in men in The Cardiovascular Risk in Young Finns Study. Atherosclerosis. 2008; 196:841-848.

17. Juonala M, Jarvisalo MJ, Maki-Torkko N, Kahonen M, Viikari JS, Raitakari OT. Risk factors identified in childhood and decreased carotid artery elasticity in adulthood: the Cardiovascular Risk in Young Finns Study. Circulation. 2005; 112:1486-1493.

18. Kampus P, Kals J, Ristimae T, Fischer K, Zilmer M, Teesalu R. High-sensitivity C-reactive protein affects central haemodynamics and augmentation index in apparently healthy persons. J Hypertens. 2004; 22:1133-1139.

19. Kampus P, Kals J, Ristimae T, Muda P, Ulst K, Zilmer K, Salonen RM, Tuomainen TP, Teesalu R, Zilmer $M$. Augmentation index and carotid intima-media thickness are differently related to age, $C$-reactive protein and oxidized low-density lipoprotein. J Hypertens. 2007; 25:819-825.

20. Kullo IJ, Seward JB, Bailey KR, Bielak LF, Grossardt BR, Sheedy PF, 2nd, Peyser PA, Turner ST. Creactive protein is related to arterial wave reflection and stiffness in asymptomatic subjects from the community. Am J Hypertens. 2005; 18:1123-1129. 
21. Mattace-Raso FU, van der Cammen TJ, van der Meer IM, Schalekamp MA, Asmar R, Hofman A, Witteman JC. C-reactive protein and arterial stiffness in older adults: the Rotterdam Study. Atherosclerosis. 2004; 176:111-116.

22. Nakhai-Pour HR, Grobbee DE, Bots ML, Muller M, van der Schouw YT. C-reactive protein and aortic stiffness and wave reflection in middle-aged and elderly men from the community. J Hum Hypertens. 2007; 21:949-955.

23. Schumacher W, Cockcroft J, Timpson NJ, McEniery CM, Gallacher J, Rumley A, Lowe G, Smith GD, Wilkinson IB, Ben-Shlomo Y. Association between C-reactive protein genotype, circulating levels, and aortic pulse wave velocity. Hypertension. 2009; 53:150-157.

24. Schutte R, Schutte AE, Van Rooyen JM, Huisman HW, Palmer IM, Fourie CM, Peter S, Malan L, Malan NT, Reimann M. Von Willebrand factor as marker of vascular function in South African women: the POWIRS Study. Am J Hypertens. 2008; 21:1298-1303.

25. Whincup PH, Gilg JA, Donald AE, Katterhorn M, Oliver C, Cook DG, Deanfield JE. Arterial distensibility in adolescents: the influence of adiposity, the metabolic syndrome, and classic risk factors. Circulation. 2005; 112:1789-1797.

26. Yasmin, McEniery CM, Wallace S, Mackenzie IS, Cockcroft JR, Wilkinson IB. C-reactive protein is associated with arterial stiffness in apparently healthy individuals. Arterioscler Thromb Vasc Biol. 2004; 24:969-974.

27. Stehouwer CD, Gall MA, Twisk JW, Knudsen E, Emeis JJ, Parving HH. Increased urinary albumin excretion, endothelial dysfunction, and chronic low-grade inflammation in type 2 diabetes: progressive, interrelated, and independently associated with risk of death. Diabetes. 2002; 51:11571165.

28. Ferreira I, Boreham CA, Twisk JW, Gallagher AM, Young IS, Murray LJ, Stehouwer CD. Clustering of metabolic syndrome risk factors and arterial stiffness in young adults: the Northern Ireland Young Hearts Project. J Hypertens. 2007; 25:1009-1020.

29. Kemper HC. Amsterdam Growth and Health Longitudinal Study (AGAHLS): A 23-Year Follow-up From Teenage to Adult About Lifestyle and Health. Basel, Switzerland: Karger; 2004.

30. van Bussel BC, Henry RM, Schalkwijk CG, Ferreira I, Feskens EJ, Streppel MT, Smulders YM, Twisk JW, Stehouwer CD. Fish consumption in healthy adults is associated with decreased circulating biomarkers of endothelial dysfunction and inflammation during a 6-year follow-up. J Nutr. 2011; 141:1719-1725.

31. Ferreira I, Henry RM, Twisk JW, van Mechelen W, Kemper HC, Stehouwer CD. The metabolic syndrome, cardiopulmonary fitness, and subcutaneous trunk fat as independent determinants of arterial stiffness: the Amsterdam Growth and Health Longitudinal Study. Arch Intern Med. 2005; 165:875-882.

32. Schouten F, Twisk JW, de Boer MR, Stehouwer CD, Serne EH, Smulders YM, Ferreira I. Increases in central fat mass and decreases in peripheral fat mass are associated with accelerated arterial stiffening in healthy adults: the Amsterdam Growth and Health Longitudinal Study. Am J Clin Nutr. 2011; 94:40-48.

33. Twisk JWR. Applied Longitudinal Data Analysis for Epidemiology: A Practical Guide. 1st ed. Cambridge, United Kingdom: Cambridge University; 2003.

34. Laurent S, Cockcroft J, Van Bortel L, Boutouyrie P, Giannattasio C, Hayoz D, Pannier B, Vlachopoulos C, Wilkinson I, Struijker-Boudier H. Expert consensus document on arterial stiffness: methodological issues and clinical applications. Eur Heart J. 2006; 27:2588-2605.

35. Aird WC. Phenotypic heterogeneity of the endothelium: I. Structure, function, and mechanisms. Circ Res. 2007; 100:158-173.

36. Aird WC. Phenotypic heterogeneity of the endothelium: II. Representative vascular beds. Circ Res. 2007; 100:174-190.

37. Deanfield J, Donald A, Ferri C, Giannattasio C, Halcox J, Halligan S, Lerman A, Mancia G, Oliver JJ, Pessina AC, Rizzoni D, Rossi GP, Salvetti A, Schiffrin EL, Taddei S, Webb DJ. Endothelial function and dysfunction. Part I: Methodological issues for assessment in the different vascular beds: a statement by the Working Group on Endothelin and Endothelial Factors of the European Society of Hypertension. J Hypertens. 2005; 23:7-17. 
38. Yeboah J, Crouse JR, Hsu FC, Burke GL, Herrington DM. Brachial flow-mediated dilation predicts incident cardiovascular events in older adults: the Cardiovascular Health Study. Circulation. 2007; 115:2390-2397.

39. Napoli C, de Nigris F, Palinski W. Multiple role of reactive oxygen species in the arterial wall. J Cell Biochem. 2001; 82:674-682.

40. Yudkin JS, Stehouwer CD, Emeis JJ, Coppack SW. C-reactive protein in healthy subjects: associations with obesity, insulin resistance, and endothelial dysfunction: a potential role for cytokines originating from adipose tissue? Arterioscler Thromb Vasc Biol. 1999; 19:972-978. 


\section{Appendix 8}

\section{Assessment of endothelial dysfunction and low-grade inflammation}

The electrochemiluminescence detection system uses multi-array plates fitted with multi-electrodes per well with each electrode being coated with a different catching antibody. The assay procedure then follows that of a classic sandwich enzyme linked immunosorbent assay (ELISA) with any of the analytes of interest captured on the relevant electrode. These captured analytes were then in turn detected by a secondary analyte-specific ruthenium-conjugated antibody, which is capable of emitting light after electrochemical stimulation. A particular advantage of this system is the ability to measure different biomarkers of endothelial dysfunction and/or lowgrade inflammation simultaneously in relatively small ( 25 or $50 \mu \mathrm{l}$ ) serum samples. In our laboratory intra- and inter-assay coefficients of variation were for sVCAM-1, 3.8 and 6.9\%; for sE-selectin, 4.1 and 8.6\%; for STM, 2.5 and 8.1\%; for sICAM-1, 2.6 and 6.0\%; for CRP, 2.3 and 4.3\%; for SAA, 4.5 and 9.0\%; for IL-6, 6.3 and 17.5\%; for IL-8, 6.9 and 7.3\%; and for TNF- $\alpha, 5.9$ and $12.6 \%$, respectively. We also measured IL-1 $1 \beta$, but levels were below the detection limit in 107 participants (resulting in intra- and interassay variation coefficients of 20.0 and $32.2 \%$, respectively), and therefore we excluded IL-1 $\beta$ from the present investigation.

For the von Willebrand factor (vWf) ELISA, vWf rabbit antibody was used as a catching antibody and a peroxidase-conjugate rabbit antibody as detecting antibody. ${ }^{1}$ Levels were expressed as a percentage of vWf detected in pooled citrated plasma of healthy volunteers. Intra- and inter-assay coefficients of variation for vWf were 3.8 and $7.4 \%$, respectively.

\section{Assessment of arterial stiffness}

Arterial stiffness of the carotid, femoral and brachial artery was determined according to international guidelines ${ }^{2}$ and the exact procedures for the AGAHLS cohort, as executed in the 2000 follow-up, have been described elsewhere. ${ }^{3}$ Briefly, arterial diameter (D), intima-media thickness (IMT) and distension ( $\Delta \mathrm{D})$ were quantified by ultrasonography ${ }^{3}$ and local pulse pressure (PP) was determined according to the Van Bortel calibration method of distension waveforms. ${ }^{4}$ Arterial stiffness was then calculated according to the following formulas:

\begin{tabular}{ll}
- & Distensibility Coefficient (DC) \\
DC $=\left(2 \Delta \mathrm{D} \cdot \mathrm{D}+\Delta \mathrm{D}^{2}\right) /\left(\mathrm{PP} \cdot \mathrm{D}^{2}\right)$ & $\left(10^{-3} \cdot \mathrm{kPa}^{-1}\right)$ \\
& $\frac{}{\mathrm{Compliance} \text { Coefficient }(\mathrm{CC})}$ \\
$\mathrm{CC}=\pi \cdot\left(2 \mathrm{D} \cdot \Delta \mathrm{D}+\Delta \mathrm{D}^{2}\right) / 4 \mathrm{PP}$ & $\left(\mathrm{mm}^{2} \cdot \mathrm{kPa}^{-1}\right)$ \\
& $\frac{}{\text { Young's elastic modulus }(\mathrm{YEM})}$ \\
\hline $\mathrm{E}_{\mathrm{inc}}=\mathrm{D} /(\mathrm{IMT} \cdot \mathrm{DC})$ & $\left(10^{3} \cdot \mathrm{kPa}\right)$
\end{tabular}


DC represents local arterial stiffness; CC represents the arterial buffering capacity; and YEM represents the intrinsic stiffness of the arterial wall.

In addition, central arterial stiffness [the carotid-femoral pulse wave velocity (cfPWV, in $\mathrm{m} / \mathrm{s}$ )] was measured by dividing the length between the carotid and the femoral arterial sites by the carotid-to-femoral transit time. The carotid-to-femoral transit time was calculated by subtracting the travel time of the pressure wave from the heart (as triggered by an electrocardiogram) to the femoral artery by that from the heart to the carotid artery. For technical reasons, cfPWV was obtained only in 241 individuals in 2000 and in 292 individuals in 2006.

\section{Calculation of overall Z-scores for endothelial dysfunction and low- grade inflammation}

For reasons of statistical efficiency and to reduce the influences of the biological variability of each measure ${ }^{5}$, overall Z-scores were determined for both endothelial dysfunction and low-grade inflammation in 2000 and 2006, according to predefined clusters of conceptually related biomarkers. ${ }^{5,6}$ These overall Z-scores were calculated as follows: first, for each individual biomarker a Z-score was calculated according to the formula: (individual value - population mean) / population standard deviation. The resulting individual Z-scores were then averaged into the overall Z-score for either endothelial dysfunction or low-grade inflammation. The endothelial dysfunction overall Z-score consisted of the biomarkers sICAM-1, sVCAM-1, sE-selectin, sTM and vWf. The low-grade inflammation overall Z-score consisted of the biomarkers CRP, SAA, IL-6, IL-8, TNF- $\alpha$ and SICAM-1. SICAM-1 levels were included in both overall Zscores as it is expressed by both monocytes and the endothelium and is strongly affected by inflammatory stimuli. ${ }^{7}$ 
Endothelium, inflammation, arterial stiffness

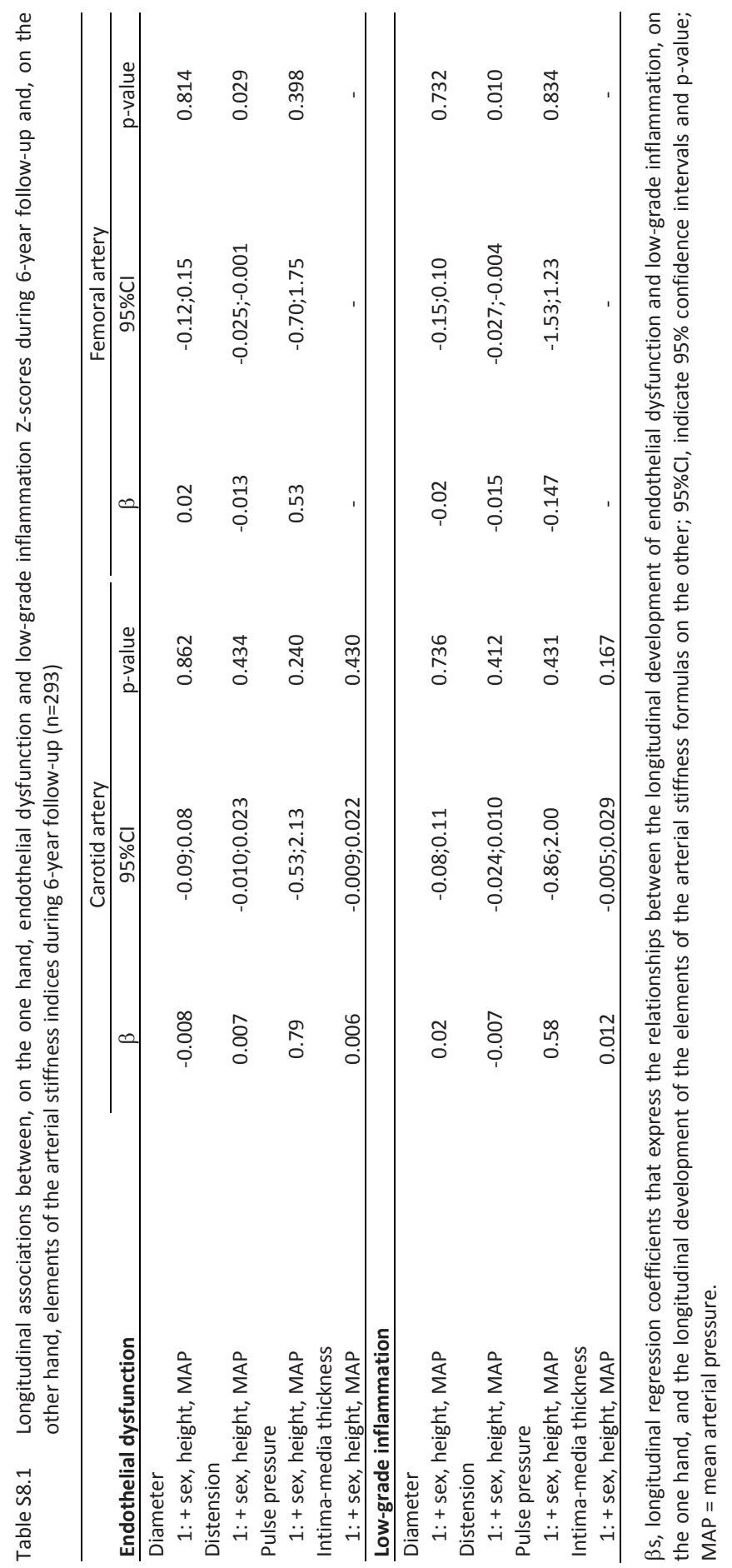




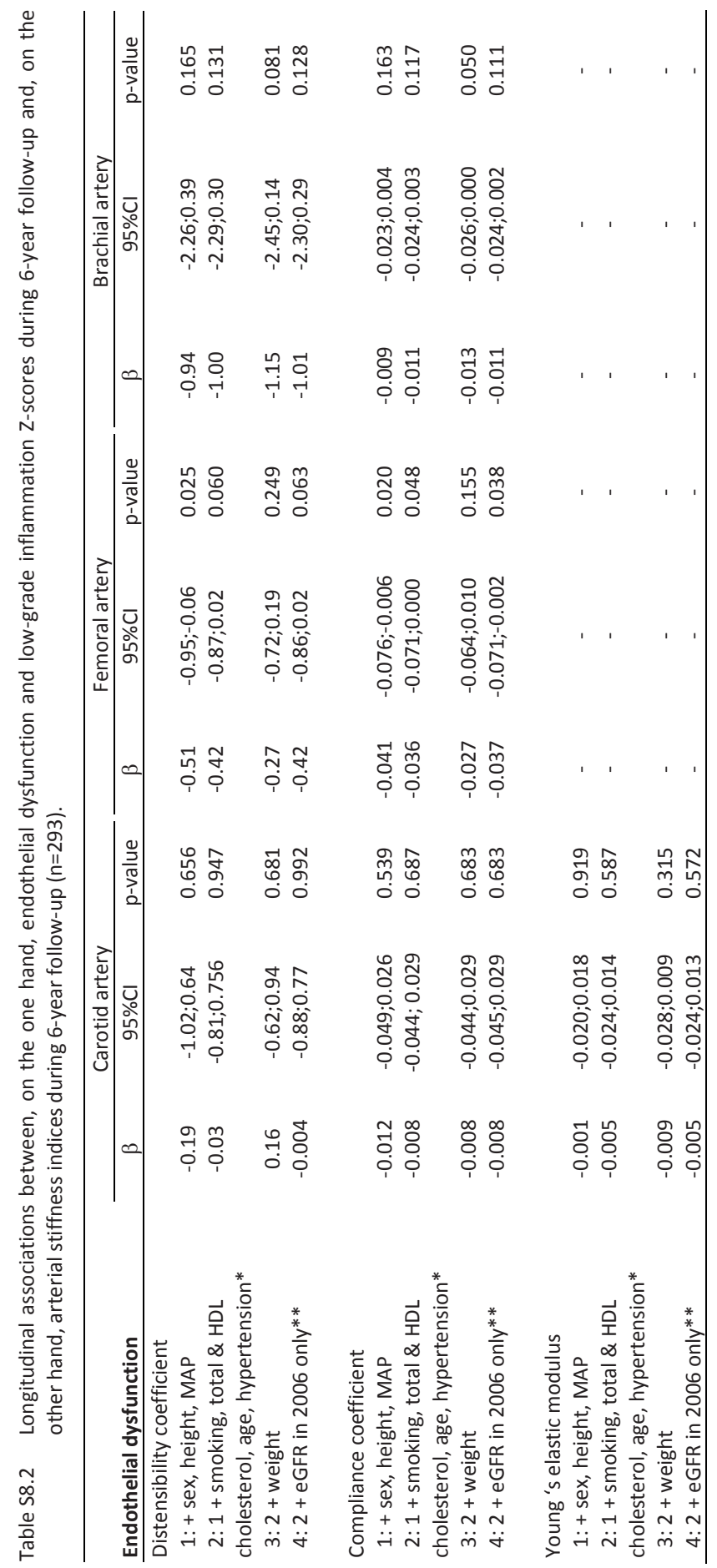




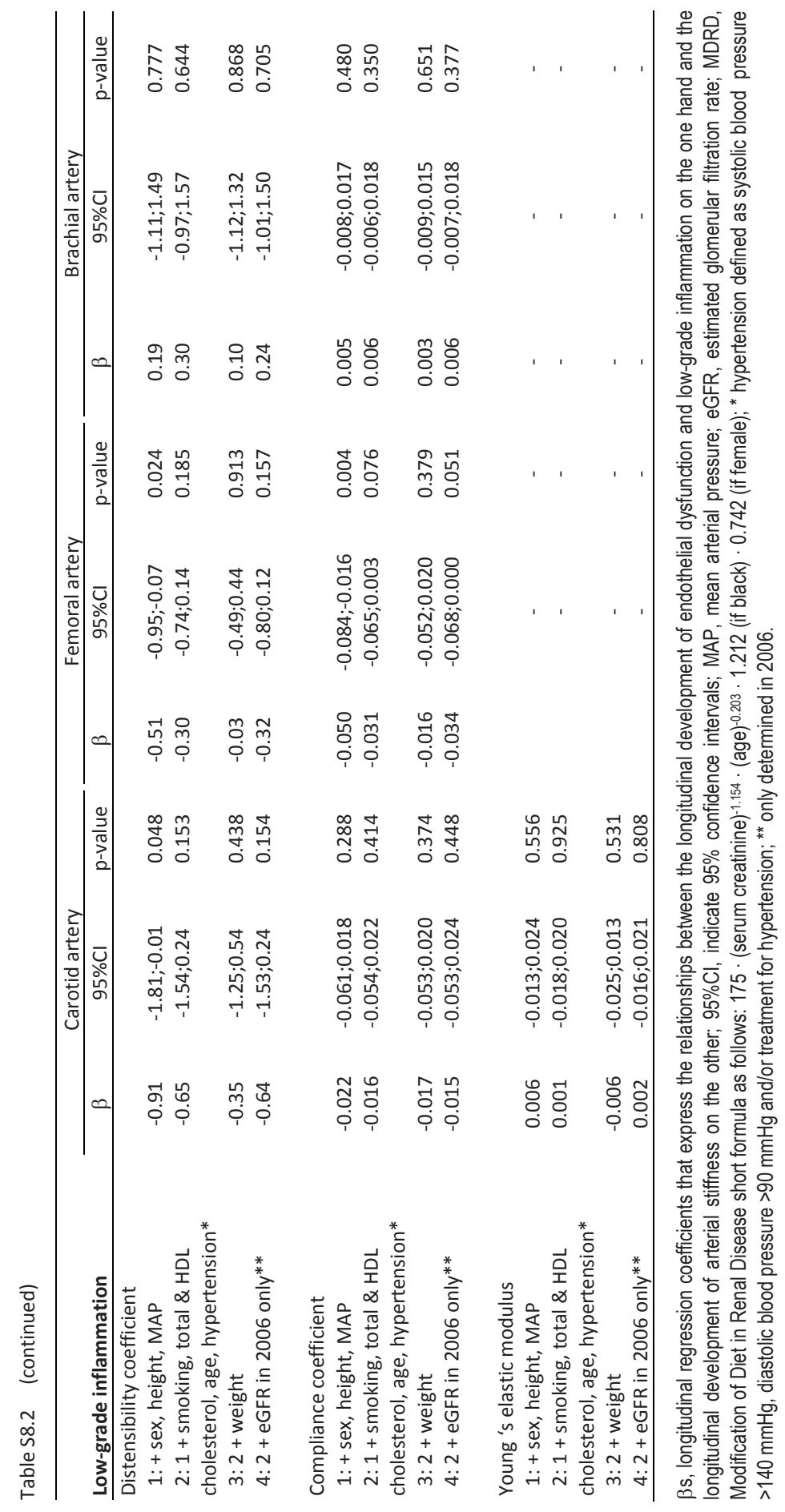




\section{References Appendix 8}

1. de Valk-de Roo GW, Stehouwer CD, Lambert J, Schalkwijk CG, van der Mooren MJ, Kluft C, Netelenbos C. Plasma homocysteine is weakly correlated with plasma endothelin and von willebrand factor but not with endothelium-dependent vasodilatation in healthy postmenopausal women. Clin Chem. 1999;45:1200-1205.

2. Laurent S, Cockcroft J, Van Bortel L, Boutouyrie P, Giannattasio C, Hayoz D, Pannier B, Vlachopoulos C, Wilkinson I, Struijker-Boudier H. Expert consensus document on arterial stiffness: Methodological issues and clinical applications. Eur Heart J. 2006;27:2588-2605.

3. Ferreira I, Henry RM, Twisk JW, van Mechelen W, Kemper HC, Stehouwer CD. The metabolic syndrome, cardiopulmonary fitness, and subcutaneous trunk fat as independent determinants of arterial stiffness: The amsterdam growth and health longitudinal study. Arch Intern Med. 2005; $165: 875-882$.

4. Van Bortel LM, Balkestein EJ, van der Heijden-Spek JJ, Vanmolkot FH, Staessen JA, Kragten JA, Vredeveld JW, Safar ME, Struijker Boudier HA, Hoeks AP. Non-invasive assessment of local arterial pulse pressure: Comparison of applanation tonometry and echo-tracking. J Hypertens. 2001;19: 1037-1044.

5. Yudkin JS, Stehouwer CD, Emeis JJ, Coppack SW. C-reactive protein in healthy subjects: Associations with obesity, insulin resistance, and endothelial dysfunction: A potential role for cytokines originating from adipose tissue? Arterioscler Thromb Vasc Biol. 1999;19:972-978.

6. de Jager J, Dekker JM, Kooy A, Kostense PJ, Nijpels G, Heine RJ, Bouter LM, Stehouwer CD. Endothelial dysfunction and low-grade inflammation explain much of the excess cardiovascular mortality in individuals with type 2 diabetes: The hoorn study. Arterioscler Thromb Vasc Biol. 2006;26:1086-1093.

7. Schram MT, Stehouwer CD. Endothelial dysfunction, cellular adhesion molecules and the metabolic syndrome. Horm Metab Res. 2005;37 Suppl 1:49-55. 


\title{
Chapter
}

\author{
Low-grade inflammation, but not endothelial \\ dysfunction, is associated with greater carotid \\ stiffness in the elderly - the Hoorn Study
}

BCT van Bussel, RMA Henry, CG Schalkwijk, JM Dekker, G Nijpels, CDA Stehouwer

J Hypertens. 2012;30:744-752

Reprinted with permission of the publisher 


\section{Abstract}

\section{Objective}

Biomarkers of low-grade inflammation and endothelial dysfunction are associated with cardiovascular disease. Arterial stiffening may be a mechanism through which low-grade inflammation and/or endothelial dysfunction lead to cardiovascular disease. Therefore, we investigated whether low-grade inflammation and endothelial dysfunction were associated with greater carotid stiffness in a population-based cohort of elderly individuals.

\section{Methods}

We determined biomarkers of low-grade inflammation (C-reactive protein, serum amyloid $A$, interleukin 6 , interleukin 8 , tumour necrosis factor $\alpha$ and soluble intercellular adhesion molecule 1), and of endothelial dysfunction (von Willebrand factor, soluble vascular cell adhesion molecule 1, soluble endothelial selectin, soluble thrombomodulin, soluble intercellular adhesion molecule 1 and flow-mediated dilation), and combined these into mean Z-scores ( $n=572$; women=286; age $67.5 \pm 6.6$ years). Additionally, we determined by ultrasonography carotid diameter, distension, pulse pressure and intima-media thickness. Carotid stiffness indices were determined by calculation of the distensibility and compliance coefficient, Young's elastic modulus and $\beta$-stiffness index.

\section{Results}

The study population was characterized by a high prevalence of cardiovascular disease (46\%), hypertension (66\%), and the use of lipid-lowering (16\%) and anti-hypertensive (34\%) medication. After adjustment for the above in addition to sex, age, glucose tolerance status and current smoking, the low-grade inflammation Z-score was positively associated with Young's elastic modulus $[\mathrm{B}(95 \% \mathrm{Cl}) 0.080(0.021 ; 0.139), p=0.008]$. This association was primarily driven through greater diameter. After adjustment for the variables above, the endothelial dysfunction Z-score was not associated with carotid stiffness.

\section{Conclusions}

These data suggest that low-grade inflammation, in the elderly, plays an important role in carotid artery remodelling and stiffening. 


\section{Introduction}

Biomarkers of low-grade inflammation and endothelial dysfunction are closely associated with (incident) cardiovascular disease (CVD). ${ }^{1-7}$ In part, these associations are explained by the fact that low-grade inflammation and endothelial dysfunction play key roles in atherothrombosis. ${ }^{8,9}$ However, other mechanisms may also be important and arterial stiffening may constitute one such mechanism.

The association between biomarkers of low-grade inflammation and/or endothelial dysfunction and greater arterial stiffness is receiving increased attention. ${ }^{10-13}$ We have previously shown that biomarkers of low-grade inflammation, but not endothelial dysfunction, were associated with greater stiffness of the carotid artery in young healthy adults. ${ }^{14}$ In addition, data on the association between low-grade inflammation and carotid stiffness in middle-aged populations show mixed results. ${ }^{15-17}$ Some report positive (univariate) associations between low-grade inflammation and carotid artery stiffness ${ }^{16,17}$, whereas others do not report such associations. ${ }^{15}$ However, no results on this association or on the association between endothelial dysfunction and carotid stiffness have been published in the elderly.

Therefore, we investigated the association between low-grade inflammation and endothelial dysfunction, on the one hand, and carotid stiffness on the other in a population-based cohort of elderly individuals.

\section{Methods}

\section{Study population}

The present investigation involves the participants from the 2000-2001 Hoorn Study follow-up examination (extensively described elsewhere). ${ }^{18-20}$ Briefly, the study population consisted of three groups: one with normal glucose tolerance (NGM), one with impaired glucose metabolism (IGM), and one with type 2 diabetes mellitus (DM2). Individuals with IGM and DM2 were oversampled to allow analyses of the role of glucose intolerance. ${ }^{18-20}$

Recently, we determined six biomarkers of low-grade inflammation and five biomarkers of endothelial dysfunction in previously stored samples of the 2000-2001 examination, whereas a sixth (functional) biomarker of endothelial dysfunction had been determined in 2000-2001 (vide infra).

The local ethics committee approved the study and all participants gave their written informed consent. 


\section{Assessment of biomarkers of low-grade inflammation and endothelial dysfunction}

Serum biomarkers of low-grade inflammation [C-reactive protein (CRP), serum amyloid A (SAA), interleukin 6 (IL-6), interleukin 8 (IL-8), tumour necrosis factor $\alpha$ (TNF- $\alpha$ ) and soluble intercellular adhesion molecule 1 (sICAM-1)], and endothelial dysfunction [soluble vascular cell adhesion molecule 1 (sVCAM-1), soluble endothelial selectin (sE-selectin), soluble thrombomodulin (sTM) and sICAM-1] were assessed by a multi-array detection system based on electrochemiluminescence technology (MesoScaleDiscovery, SECTOR Imager 2400, Gaithersburg, MD, USA). Briefly, this system uses multi-array plates fitted with multi-electrodes per well with each electrode being coated with a different catching antibody. The assay procedure then follows that of a classic sandwich ELISA with any of the analytes of interest captured on the relevant electrode. These captured analytes were then in turn detected by a secondary analyte-specific ruthenium-conjugated antibody, which is capable of emitting light after electrochemical stimulation. A particular advantage of this system is the ability to measure different biomarkers of low-grade inflammation and/or endothelial dysfunction simultaneously in relatively small (25 or $50 \mu \mathrm{l}$ ) serum samples. In our laboratory, intra- and inter-assay coefficients of variation were, respectively, 2.8 and $4.0 \%$ for CRP; 2.7 and $11.6 \%$ for SAA; 5.6 and $13.0 \%$ for IL-6, 5.6 and $12.2 \%$ for IL-8, 3.9 and 8.8\% for TNF- $\alpha, 2.4$ and $4.9 \%$ for sICAM-1, 2.8 and 5.6\% for SVCAM-1, 2.6 and $6.7 \%$ for sE-selectin 2.1 and $6.9 \%$ for sTM. We also measured IL-1 $\beta$ using the above system, but levels were below the detection limit in 191 participants (resulting in intra- and inter-assay variation coefficients of 24.3 and $36.9 \%$, respectively). We therefore excluded IL-1 $\beta$ from the present investigation.

Two additional biomarkers of endothelial dysfunction were determined. First, von Willebrand factor (vWf) was determined in citrated plasma by means of ELISA. Briefly, vWf rabbit antibody was used as a catching antibody and a peroxidase-conjugate rabbit antibody as detecting antibody. ${ }^{21}$ Levels were expressed as a percentage of vWf detected in pooled citrated plasma of healthy volunteers. Intra- and inter-assay coefficients of variation for vWf were 3.4 and $7.9 \%$, respectively. Second, a functional biomarker of endothelial dysfunction [flow-mediated dilation (FMD)] was measured in the right brachial artery as described elsewhere. ${ }^{22}$

\section{Arterial stiffness}

Carotid stiffness was determined according to international guidelines ${ }^{23}$ and the exact procedures and reproducibility for the Hoorn Study cohort have been described elsewhere. $^{20}$

Briefly, carotid diameter (D), intima-media thickness (IMT) and distension ( $\triangle D$ ) were quantified by ultrasonography, systolic (SBP) and diastolic (DBP) blood pressure were measured and carotid pulse pressure (PP) was determined according to the van Bortel 
calibration method. ${ }^{24}$ Carotid stiffness was then calculated according to the following formulas:

- $\quad$ Distensibility Coefficient (DC)

$$
D C=\left(2 \Delta D \cdot D+\Delta D^{2}\right) /\left(P P \cdot D^{2}\right) \quad\left(10^{-3} \cdot \mathrm{kPa}^{-1}\right)
$$

- $\quad$ Compliance Coefficient (CC)

$$
\mathrm{CC}=\pi \cdot\left(2 \mathrm{D} \cdot \Delta \mathrm{D}+\Delta \mathrm{D}^{2}\right) / 4 \mathrm{PP} \quad\left(\mathrm{mm}^{2} \cdot \mathrm{kPa}^{-1}\right)
$$

- Young's elastic modulus (YEM)

$$
\mathrm{E}_{\mathrm{inc}}=\mathrm{D} /(\mathrm{IMT} \cdot \mathrm{DC})
$$

- $\quad \underline{\beta}$-stiffness index (SI)

$$
S I=\ln (S B P / D B P) /(\triangle D / D)
$$

Distensibility coefficient (DC) represents carotid wall stiffness, compliance coefficient (CC) represents the carotid buffering capacity, Young's elastic modulus (YEM) represents the intrinsic stiffness of the carotid wall and $\beta$-stiffness index (SI) approximates the nonlinear relation between pressure and diameter.

\section{Other measurements}

Health status, medical history, medication use and smoking habits were assessed by questionnaire. Height, weight, waist and hip circumference, SBP and DBP, serum total cholesterol, high-density lipoprotein (HDL) and low-density lipoprotein (LDL) cholesterols, serum triglycerides, glucose, insulin, glycated haemoglobin, creatinine, albuminuria and prior cardiovascular disease were determined as described elsewhere. $^{18-20}$

We calculated BMI and waist-to-hip ratio. Smoking behaviour was dichotomized into current smokers and non-smokers; hypertension was dichotomized and defined as a SBP at least $140 \mathrm{mmHg}$ and DBP at least $90 \mathrm{mmHg}$ and/or currently used antihypertensive medication. Estimated glomerular filtration rate (eGFR) $\left(\mathrm{ml} / \mathrm{min} / 1.73 \mathrm{~m}^{2}\right.$ ) was calculated according to Levey's [Modification of Diet in Renal Disease (MDRD)] short formula as follows: $186 \cdot(\text { serum creatinine) })^{-1.154} \cdot(\text { age })^{-0.203} \cdot 1.212$ (if black) 0.742 (if female). ${ }^{25}$

\section{Statistical analyses}

All analyses were performed with SPSS (Statistical Package for Social Sciences, SPSS Inc, version 15.0, Chicago, Illinois, USA). Outcome variables with a skewed distribution were natural log-transformed in order to meet normality criteria (serum triglycerides, insulin, CRP, SAA, IL-6 and IL-8). 
For reasons of statistical efficiency and to reduce the influence of the biological variability of each measure, an overall Z-score was determined for both low-grade inflammation and endothelial dysfunction, according to predefined clusters of conceptually related biomarkers. ${ }^{3,26}$ The overall Z-scores were calculated as follows: for each individual biomarker, a Z-score was calculated according to the formula: (individual value - population mean) / population standard deviation. The resulting individual biomarker Z-scores were then averaged into the overall Z-score for both low-grade inflammation and endothelial dysfunction. The low-grade inflammation overall Z-score consisted of the biomarkers CRP, SAA, IL-6, IL-8, TNF- $\alpha$ and SICAM-1; and the endothelial dysfunction overall Z-score consisted of the biomarkers vWf, sE-selectin, sTM, sVCAM-1, FMD (FMD was inversed so that higher values indicated worse function) and SICAM-1. SICAM-1 was included in both overall Z-scores as it is expressed by both monocytes and the endothelium. ${ }^{27}$

We used linear regression analyses to investigate the associations between the overall Z-scores for low-grade inflammation and endothelial dysfunction, on the one hand, and the carotid DC, CC, YEM and SI on the other. First, these associations were adjusted for sex and age (models 1 ) and then, additionally, for glucose tolerance status (models 2), for mean arterial pressure (models 3 ) and for anti-hypertensive and lipid-lowering medication, smoking behaviour and prior cardiovascular disease (models 4). In addition, associations between low-grade inflammation or endothelial dysfunction and carotid stiffness (if any) were mutually adjusted for each of the overall Z-scores (models 5). Finally, to gain further insight into which of the individual elements of the stiffness formulas might have been primarily responsible for the relationships (if any) between low-grade inflammation or endothelial dysfunction and carotid stiffness indices, the analyses were repeated with the individual elements of the stiffness formulas ( $D, \Delta D, P P$ and IMT) as dependent variables. In addition, we used interaction terms to explore whether associations differed by glucose tolerance status.

Data are presented as mean $( \pm S D)$, median (interquartile range) for skewed variables or as percentages and regression coefficients $(\beta)$ with their $95 \%$ confidence intervals $(95 \% \mathrm{Cl})$. A two-sided $\mathrm{p}$-value less than 0.05 and a $\mathrm{p}_{\text {(interaction) }}$ less than 0.10 were considered statistically significant.

\section{Results}

Of the 747 participants who underwent carotid ultrasonography, 79 did not have their carotid PP estimated and three did not undergo an oral glucose tolerance test. In the remaining 665 participants, all serum and plasma biomarkers were determined in 601 individuals. The main reason for missing data was missing serum $(n=28)$, plasma $(n=7)$ or serum and plasma $(n=29)$ samples. Of these 601 participants, 572 qualitatively satisfactory FMDs were obtained. Full data were therefore available in 572 individuals. 


\section{General characteristics according to tertiles of low-grade inflammation}

Ninety-one percentage of the study population were $>60$ (range 50-86) years of age. Table 9.1 shows that, as compared with individuals in the lowest tertile of the lowgrade inflammation overall Z-score, those in the middle and highest tertiles had a worse glucose tolerance status, a lower eGFR, a higher blood pressure and higher prevalences of CVD, smoking and albuminuria.

Table 9.1 Clinical characteristics according to tertiles of the low-grade inflammation score.

\begin{tabular}{|c|c|c|c|c|}
\hline Clinical characteristics & $\begin{array}{c}\mathrm{T} 1 \\
\mathrm{n}=190\end{array}$ & $\begin{array}{c}\mathrm{T} 2 \\
\mathrm{n}=191\end{array}$ & $\begin{array}{c}T 3 \\
n=191\end{array}$ & $\mathrm{p}$ (trend) \\
\hline No., $M / F$ & $102 / 88$ & $97 / 94$ & $87 / 104$ & 0.113 \\
\hline Age, years & $66.0 \pm 6.1$ & $68.2 \pm 6.9$ & $68.3 \pm 6.6$ & 0.001 \\
\hline \multicolumn{5}{|l|}{ Glucose tolerance status * } \\
\hline Normal glucose tolerance, $\%$ & 50.0 & 37.7 & 34.0 & \\
\hline Impaired glucose metabolism, \% & 17.9 & 26.2 & 22.5 & \\
\hline Diabetes mellitus type $2, \%$ & 32.1 & 36.1 & 43.5 & 0.003 \\
\hline Serum insulin, $\mathrm{pmol} / \mathrm{l}+$ & $51.0(39.0-70.0)$ & $59.0(39.0-88.5)$ & $67.0(47.0-97.5)$ & $<0.001$ \\
\hline Glycated haemoglobin, \% & $5.8 \pm 0.7$ & $6.0 \pm 0.6$ & $6.2 \pm 0.8$ & $<0.001$ \\
\hline IFCC- $\mathrm{HbA} 1 \mathrm{c}, \mathrm{mmol} / \mathrm{mol}$ & $40.2 \pm 7.2$ & $42.0 \pm 6.5$ & $44.5 \pm 8.9$ & $<0.001$ \\
\hline Body mass index, $\mathrm{kg} / \mathrm{m}^{2}$ & $26.3 \pm 3.2$ & $27.5 \pm 3.5$ & $27.4 \pm 3.5$ & 0.003 \\
\hline Waist-to-hip ratio & $0.92 \pm 0.12$ & $0.92 \pm 0.09$ & $0.94 \pm 0.09$ & 0.110 \\
\hline Brachial systolic pressure, $\mathrm{mmHg}$ & $137.1 \pm 18.0$ & $141.7 \pm 17.7$ & $145.2 \pm 20.6$ & $<0.001$ \\
\hline Brachial diastolic pressure, $\mathrm{mmHg}$ & $75.5 \pm 8.5$ & $77.2 \pm 8.8$ & $78.1 \pm 9.8$ & 0.005 \\
\hline Hypertension, \% & 62.2 & 66.5 & 68.1 & 0.233 \\
\hline Anti-hypertensive medication, $\%$ & 24.9 & 34.6 & 41.4 & 0.001 \\
\hline Prior cardiovascular disease, $\%$ & 41.5 & 45.5 & 52.2 & 0.039 \\
\hline Serum creatinine, $\mu \mathrm{mol} / \mathrm{l}$ & $93.3 \pm 13.7$ & $94.1 \pm 13.9$ & $96.2 \pm 20.6$ & 0.075 \\
\hline $\begin{array}{l}\text { Estimated glomerular filtration rate, } \\
\mathrm{ml} / \mathrm{min} / 1.73 \mathrm{~m}^{2} \ddagger\end{array}$ & $66.7 \pm 10.5$ & $65.0 \pm 9.5$ & $63.2 \pm 11.0$ & 0.001 \\
\hline Total serum cholesterol, $\mathrm{mmol} / \mathrm{l}$ & $5.7 \pm 1.1$ & $5.9 \pm 1.0$ & $5.7 \pm 1.1$ & 0.794 \\
\hline Serum HDL, mmol/l & $1.5 \pm 0.4$ & $1.4 \pm 0.4$ & $1.4 \pm 0.4$ & 0.004 \\
\hline Serum LDL, mmol// & $3.6 \pm 1.0$ & $3.8 \pm 0.8$ & $3.6 \pm 1.0$ & 0.871 \\
\hline Serum triglycerides, $\mathrm{mmol} / \mathrm{l}$ & $1.2(0.9-1.6)$ & $1.4(1.0-1.9)$ & $1.5(1.0-2.1)$ & $<0.001$ \\
\hline Lipid-lowering medication, \% & 17.5 & 13.1 & 16.2 & 0.744 \\
\hline Current smoking, \% & 9.0 & 14.7 & 20.9 & 0.001 \\
\hline $\begin{array}{l}\text { Albuminuria [albumin/creatinine ratio } \\
>2(\mathrm{mg} / \mathrm{mmol})], \%\end{array}$ & 9.5 & 12.0 & 18.3 & 0.011 \\
\hline
\end{tabular}

Data are presented as means \pm standard deviation, median (interquartile range) or percentages deviation according to tertiles (T1-3) of the low-grade inflammation overall Z-score; $p$-value for linear trend or $p$-value for linear-by-linear association as appropriate. ${ }^{*}$ normal glucose tolerance: fasting plasma glucose $<6.1$ $\mathrm{mmol} / \mathrm{l}$ and 2-hour post-load plasma glucose $<7.8 \mathrm{mmol} / \mathrm{l}$; impaired glucose metabolism: impaired fasting plasma glucose between $6.1 \mathrm{mmol} / \mathrm{l}$ and $7.0 \mathrm{mmol} / \mathrm{l}$ and impaired glucose tolerance defined as a 2-hour post-load plasma glucose between 7.8 and $11.1 \mathrm{mmol} / \mathrm{l}$; diabetes mellitus type 2: fasting plasma glucose $\geq 7.0 \mathrm{mmol} / \mathrm{l}$; and/or a 2-hour post-load plasma glucose $\geq 11.1 \mathrm{mmol} / \mathrm{l}$. + includes 4 individuals who injected insulin. $¥$ according to Levey's [Modification of Diet in Renal Disease (MDRD)] short formula before calibration: $186 \cdot\left(\right.$ serum creatinine) ) $^{-1.154} \cdot(\text { age })^{-0.203} \cdot 1.212$ (if black) 0.742 (if female); to convert to International System units multiply creatinine in $\mathrm{mg} / \mathrm{dl}$ by 88.4 for each of the equations. ${ }^{25}$ 


\section{Carotid stiffness, endothelial dysfunction and individual biomarkers according to tertiles of low-grade inflammation}

Table 9.2 shows that, as compared with individuals in the lowest tertile of the lowgrade inflammation overall Z-score, those in the middle and highest tertiles had greater carotid stiffness (i.e. a lower DC and CC and a higher YEM and SI, all indicating greater stiffness). In addition, these individuals had a higher endothelial dysfunction overall Z-score; higher levels of each of the individual biomarkers (all indicating worse function); and less FMD.

Table 9.2 Carotid stiffness, inflammation and endothelial dysfunction according to tertiles of the lowgrade inflammation Z-score.

\begin{tabular}{|c|c|c|c|c|}
\hline Low-grade inflammation & $\begin{array}{c}\mathrm{T} 1 \\
\mathrm{n}=190\end{array}$ & $\begin{array}{c}\mathrm{T} 2 \\
\mathrm{n}=191\end{array}$ & $\begin{array}{c}\text { T3 } \\
n=191\end{array}$ & $\mathrm{p}$ (trend) \\
\hline \multicolumn{5}{|l|}{ Carotid stiffness } \\
\hline $\begin{array}{l}\text { Distensibility coefficient, } \\
10^{-3} / \mathrm{kPa}\end{array}$ & $12.7 \pm 4.2$ & $11.6 \pm 4.3$ & $11.1 \pm 4.7$ & $<0.001$ \\
\hline $\begin{array}{l}\text { Compliance coefficient, } \\
\mathrm{mm}^{2} / \mathrm{kPa}\end{array}$ & $0.58 \pm 0.23$ & $0.57 \pm 0.23$ & $0.54 \pm 0.22$ & 0.076 \\
\hline $\begin{array}{l}\text { Young's elastic modulus, } \\
10^{3} * \mathrm{kPa}\end{array}$ & $0.83 \pm 0.36$ & $0.96 \pm 0.47$ & $1.08 \pm 0.65$ & $<0.001$ \\
\hline$\beta$-stiffness index & $13.8 \pm 4.8$ & $15.0 \pm 5.7$ & $16.1 \pm 7.4$ & $<0.001$ \\
\hline $\begin{array}{l}\text { Low-grade inflammation } \\
\text { Z-score, SD }\end{array}$ & $-0.59 \pm 0.24$ & $-0.08 \pm 0.13$ & $0.67 \pm 0.56$ & by definition \\
\hline $\begin{array}{l}\text { Endothelial dysfunction } \\
\text { Z-score, SD }\end{array}$ & $-0.30 \pm 0.42$ & $-0.04 \pm 0.45$ & $0.34 \pm 0.60$ & $<0.001$ \\
\hline C-reactive protein, mg/l & $1.0(0.6-1.6)$ & $2.1(1.3-3.6)$ & $4.8(2.6-9.9)$ & $<0.001$ \\
\hline Serum amyloid A, mg/l & $1.1(0.7-1.7)]$ & $1.6(1.0-2.5)$ & $3.1(1.9-6.0)$ & $<0.001$ \\
\hline Interleukin-6, ng/l & $1.0(0.8-1.4)$ & $1.3(1.1-1.9)$ & $2.2(1.6-3.5)$ & $<0.001$ \\
\hline Interleukin-8, ng/l & $12.0(9.3-15.0)$ & $14.2(11.7-18.8)$ & $16.2(12.7-21.9)$ & $<0.001$ \\
\hline $\begin{array}{l}\text { Tumour necrosis factor } \alpha \text {, } \\
\mathrm{ng} / \mathrm{l}\end{array}$ & $7.2 \pm 1.6$ & $8.4 \pm 1.9$ & $10.6 \pm 3.5$ & $<0.001$ \\
\hline $\begin{array}{l}\text { Soluble intercellular adhesion } \\
\text { molecule } 1, \mu \mathrm{g} / \mathrm{l}\end{array}$ & $218 \pm 35$ & $252 \pm 41$ & $303 \pm 68$ & $<0.001$ \\
\hline von Willebrand factor, \% & $138 \pm 42$ & $150 \pm 49$ & $163 \pm 57$ & $<0.001$ \\
\hline $\begin{array}{l}\text { Soluble endothelial selectin, } \\
\mu \mathrm{g} / \mathrm{l}\end{array}$ & $16.1 \pm 6.1$ & $19.2 \pm 7.3$ & $22.3 \pm 9.4$ & $<0.001$ \\
\hline $\begin{array}{l}\text { Soluble thrombomodulin, } \\
\mu \mathrm{g} / \mathrm{l}\end{array}$ & $3.35 \pm 0.73$ & $3.49 \pm 0.87$ & $3.58 \pm 0.87$ & 0.006 \\
\hline $\begin{array}{l}\text { Soluble vascular cellular } \\
\text { adhesion molecule } 1, \mu \mathrm{g} / \mathrm{l}\end{array}$ & $376 \pm 71$ & $392 \pm 72$ & $445 \pm 104$ & $<0.001$ \\
\hline Flow-mediated dilation, $\mathrm{mm}$ & $0.19 \pm 0.20$ & $0.18 \pm 0.18$ & $0.16 \pm 0.16$ & 0.080 \\
\hline
\end{tabular}

Data are presented as means \pm standard deviation according to tertiles (T1-3) of the low-grade inflammation overall Z-score; $\mathrm{p}$-value for linear trend. 


\section{Associations between low-grade inflammation, endothelial dysfunction and carotid stiffness}

After adjustment for sex and age, the low-grade inflammation overall Z-score was positively associated with carotid YEM $[\beta(95 \% \mathrm{Cl}) 0.130(0.066 ; 0.194), p<0.001]$ and carotid SI $[0.75(0.02 ; 1.48)$ p=0.044 (Table 9.3, models 1)]. These associations were somewhat attenuated after adjustment for glucose tolerance status and lost statistical significance for SI (models 2). With regard to YEM, additional adjustments for mean arterial pressure further attenuated this association $[0.085(0.027 ; 0.144), p=0.004$, model 3]. Further adjustment for anti-hypertensive and lipid-lowering medication, current smoking and prior CVD [0.080 (0.021;0.139), $\mathrm{p}=0.008$, model 4$]$ or for the endothelial dysfunction overall Z-score [0.083 (0.013;0.153), $p=0.020$; model 5] did not materially change this association.

Table 9.3 Associations between low-grade inflammation (CRP, SAA, IL-6, IL-8, TNF- $\alpha$ and sICAM-1) or endothelial dysfunction (vWf, sE-selectin, sTM, sVCAM-1, FMD and sICAM-1) Z-scores and carotid stiffness indices.

\begin{tabular}{lc}
\hline Low-grade inflammation & Carotid artery \\
Model & $\beta(95 \% \mathrm{Cl}), \mathrm{p}$-value \\
\hline Young 's elastic modulus & \\
1: + sex, age & $0.130(0.066 ; 0.194) 0.000$ \\
2: 1 + glucose tolerance status & $0.105(0.042 ; 0.167) 0.001$ \\
3: 2 + mean arterial pressure & $0.085(0.027 ; 0.144) 0.004$ \\
4: 3 + anti-hypertensive medication, lipid-lowering medication, & $0.080(0.021 ; 0.139) 0.008$ \\
$\quad$ current smoking, prior CVD & \\
5: 3 + endothelial dysfunction overall Z-score & $0.083(0.013 ; 0.153) 0.020$ \\
$\beta$-stiffness index & \\
1: + sex, age & $0.75(0.02 ; 1.48) 0.044$ \\
2: $1+$ glucose tolerance status & $0.47(-0.25 ; 1.19) 0.204$ \\
3: 2 + mean arterial pressure & $0.36(-0.35 ; 1.08) 0.316$ \\
4: 3 + anti-hypertensive medication, lipid-lowering medication, & $0.26(-0.46 ; 0.98) 0.483$ \\
$\quad$ current smoking, prior CVD & \\
Distensibility coefficient & \\
1: + sex, age & $-0.38(-0.91 ; 0.16) 0.169$ \\
2: $1+$ glucose tolerance status & $-0.11(-0.62 ; 0.41) 0.692$ \\
3: 2 + mean arterial pressure & $0.09(-0.38 ; 0.55) 0.716$ \\
4: 3 + anti-hypertensive medication, lipid-lowering medication, & $0.14(-0.34 ; 0.61) 0.573$ \\
$\quad$ current smoking, prior CVD & \\
Compliance coefficient & \\
1: + sex, age & \\
2: 1 + glucose tolerance status & \\
3: 2 + mean arterial pressure & \\
4: 3 + anti-hypertensive medication, lipid-lowering medication, & $0.002(-0.030 ; 0.025) 0.867$ \\
$\quad$ current smoking, prior CVD & $0.005(-0.022 ; 0.032) 0.706$ \\
\hline
\end{tabular}


Table 9.3 (continued)

\begin{tabular}{|c|c|}
\hline Endothelial dysfunction & Carotid artery \\
\hline Model & $\beta(95 \% \mathrm{Cl}), \mathrm{p}$-value \\
\hline \multicolumn{2}{|l|}{ Young 's elastic modulus } \\
\hline 1: + sex, age & $0.173(0.101 ; 0.246) 0.000$ \\
\hline 2: $1+$ glucose tolerance status & $0.124(0.051 ; 0.197) 0.001$ \\
\hline $3: 2+$ mean arterial pressure & $0.059(-0.011 ; 0.129) 0.096$ \\
\hline $\begin{array}{l}\text { 4: } 3 \text { + anti-hypertensive medication, lipid-lowering medication, current } \\
\text { smoking, prior CVD }\end{array}$ & $0.051(-0.020 ; 0.122) 0.157$ \\
\hline \multicolumn{2}{|l|}{$\beta$-stiffness index } \\
\hline $1:+$ sex, age & $1.17(0.33 ; 2.01) 0.006$ \\
\hline 2: $1+$ glucose tolerance status & $0.62(-0.22 ; 1.46) 0.150$ \\
\hline $3: 2+$ mean arterial pressure & $0.27(-0.58 ; 1.11) 0.537$ \\
\hline $\begin{array}{l}\text { 4: } 3 \text { + anti-hypertensive medication, lipid-lowering medication, current } \\
\text { smoking, prior CVD }\end{array}$ & $0.15(-0.71 ; 1.00) 0.736$ \\
\hline \multicolumn{2}{|l|}{ Distensibility coefficient } \\
\hline 1: + sex, age & $-1.18(-1.79 ;-0.57) 0.000$ \\
\hline 2: $1+$ glucose tolerance status & $-0.69(-1.29 ;-0.09) 0.025$ \\
\hline $3: 2+$ mean arterial pressure & $-0.03(-0.59 ; 0.52) 0.905$ \\
\hline $\begin{array}{l}\text { 4: } 3 \text { + anti-hypertensive medication, lipid-lowering medication, current } \\
\text { smoking, prior CVD }\end{array}$ & $0.01(-0.55 ; 0.57) 0.977$ \\
\hline \multicolumn{2}{|l|}{ Compliance coefficient } \\
\hline $1:+$ sex, age & $-0.048(-0.079 ;-0.017) 0.002$ \\
\hline 2: $1+$ glucose tolerance status & $-0.034(-0.066 ;-0.003) 0.033$ \\
\hline $3: 2+$ mean arterial pressure & $-0.017(-0.049 ; 0.014) 0.281$ \\
\hline $\begin{array}{l}\text { 4: } 3 \text { + anti-hypertensive medication, lipid-lowering medication, current } \\
\text { smoking, prior CVD }\end{array}$ & $-0.023(-0.054 ; 0.009) 0.168$ \\
\hline
\end{tabular}

Regression coefficient $(\beta)$, 95\% confidence intervals $(95 \% \mathrm{Cl})$ and $p$-value; $\beta$ represents the change in carotid stiffness index per 1 SD increase in low-grade inflammation or endothelial dysfunction Z-score; CVD, cardiovascular disease $(n=572)$.

After adjustment for sex and age, the endothelial dysfunction overall Z-score was positively associated with carotid YEM $[0.173(0.101 ; 0.246), \mathrm{p}<0.001]$ and carotid SI [1.17 $(0.33 ; 2.01), p=0.006]$, and inversely associated with carotid DC [-1.18 (1.79;$0.57), p<0.001]$ and carotid CC [-0.048 (-0.079;-0.017), $\mathrm{p}=0.002$ (Table 9.3 models 1$)]$. These associations were somewhat attenuated after adjustment for glucose tolerance status and lost statistical significance for SI (models 2). Additional adjustment for mean arterial pressure further attenuated these associations (models 3; for carotid YEM, it was $0.059(-0.011 ; 0.129), p=0.096)$. Further adjustment for anti-hypertensive and lipid-lowering medication, current smoking and prior CVD did not materially change this association for carotid YEM [0.051 (-0.020;0.122), $p=0.157$ (model 4)]. However, after adjustment for the low-grade inflammation overall Z-score, this association for carotid YEM disappeared [0.005 (-0.078;0.088), $p=0.903$ (model 5)]. 


\section{Associations between low-grade inflammation and elements of the carotid stiffness indices}

The positive association between the low-grade inflammation overall Z-score and carotid YEM was primarily driven through a greater carotid diameter (Table 9.4).

\section{Associations between low-grade inflammation, endothelial dysfunction and carotid Young's elastic modulus stratified according to glucose tolerance status}

Stratified results showed that the associations between the low-grade inflammation overall Z-score and carotid YEM and between the endothelial dysfunction overall Z-score and carotid YEM were stronger for individuals with type 2 diabetes mellitus ( $p_{\text {interaction }}=0.008$ and $=0.022$, respectively). Mutual adjustment for each of the overall Z-scores of these associations showed that only the low-grade inflammation overall Z-score, but not the endothelial dysfunction overall Z-score, was independently associated with carotid YEM in individuals with type 2 diabetes mellitus (Table 9.5).

\section{Additional analyses}

The results did not materially change if we repeated the analyses on 601 individuals including soluble biomarkers only (i.e. taking FMD out of the endothelial dysfunction overall Z-score); or if we repeated the analyses taking sICAM-1 out of either of the overall Z-scores (data not shown).

Analyses of the individual biomarkers showed that all biomarkers of the low-grade inflammation overall Z-score, except for TNF- $\alpha$, were positively associated with carotid YEM, with significant associations for SAA, CRP and IL-6 (Figure 9.1). With regard to the carotid diameter these analyses showed positive associations for all biomarkers of the low-grade inflammation overall Z-score, except for TNF- $\alpha$ and SAA. No statistically significant associations were observed for any of the individual biomarkers and carotid distension, local pulse pressure or intima-media thickness, except for an inverse association between SAA and intima-media thickness (data not shown). 


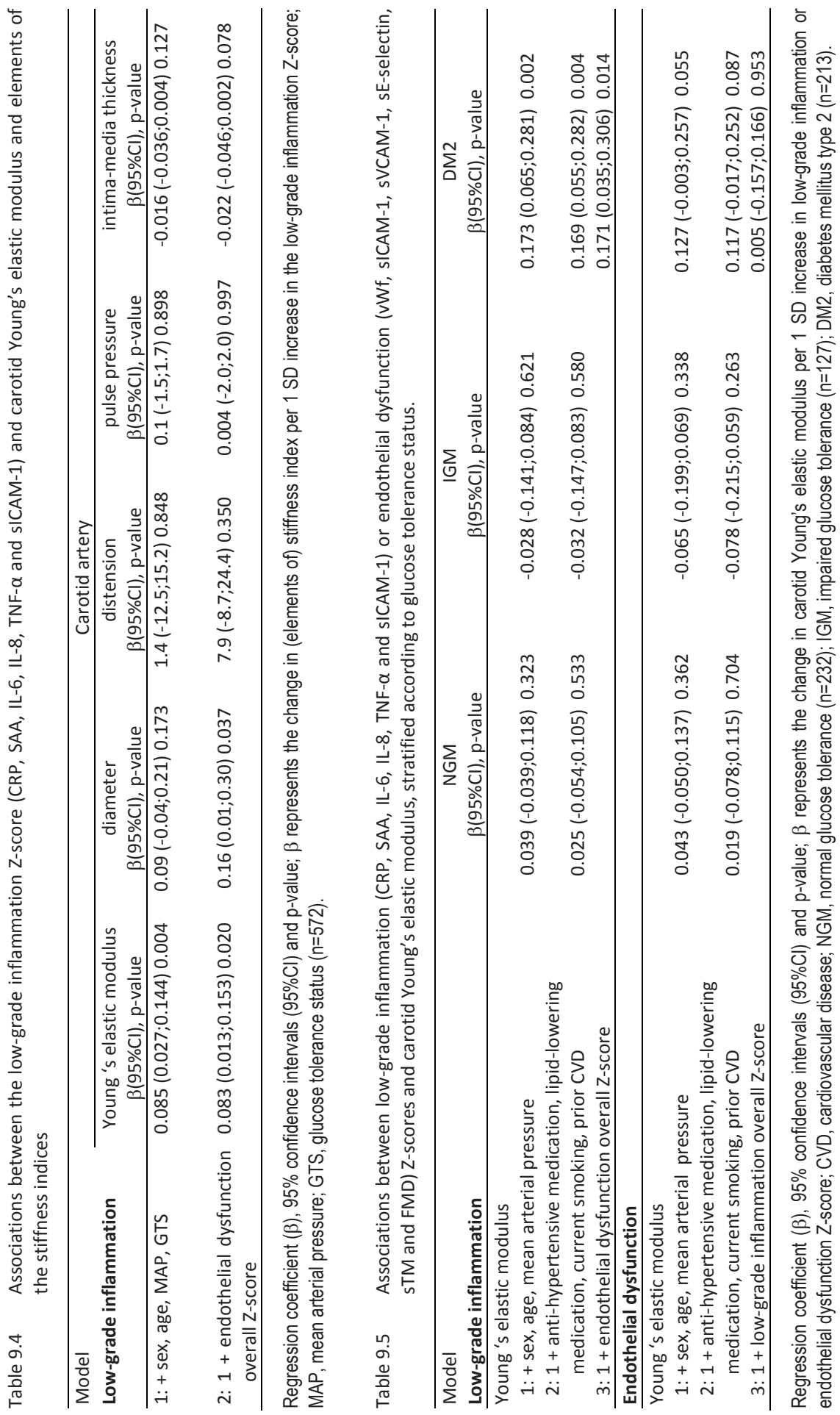




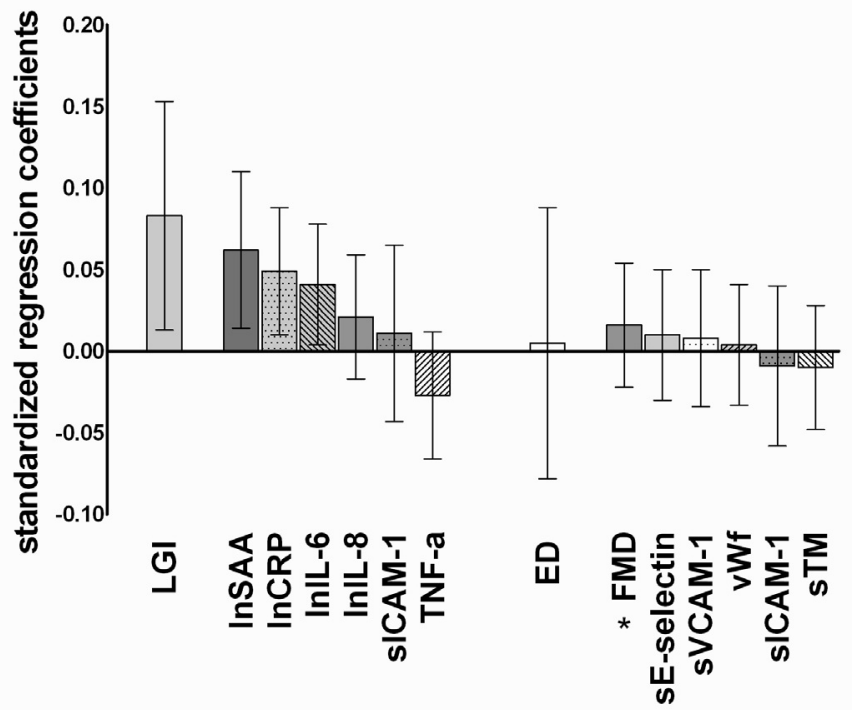

Figure 9.1 Associations between low-grade inflammation (LGI), endothelial dysfunction (ED) and individual biomarker Z-scores and carotid Young's elastic modulus.

Bars represent the change carotid Young's elastic modulus per 1 standard deviation greater LGI, ED or individual biomarker Z-scores. Results for LGI are adjusted for sex, age, mean arterial pressure, glucose tolerance status and ED, whereas results for ED are adjusted for sex, age, mean arterial pressure, glucose tolerance status and LGI $(n=572)$; whiskers indicate 95\% confidence intervals; In, log normalized; SAA, serum amyloid A; CRP, C-reactive protein; IL-6, interleukin 6; IL-8, interleukin 8; sICAM-1, soluble intercellular adhesion molecule 1; TNF- $\alpha$, tumour necrosis factor $\alpha$; FMD, flow-mediated dilation *(FMD was inversed; a higher value indicating worse function); sE-selectin, soluble endothelial selectin; sVCAM-1, soluble vascular cell adhesion molecule 1 ; vWf, von Willebrand factor; sTM, soluble thrombomodulin.

\section{Discussion}

The present investigation is the first to evaluate, in an elderly population, the relationships between six biomarkers of low-grade inflammation and six biomarkers of endothelial dysfunction, on the one hand, and four indices of carotid stiffness on the other. The study investigated elderly individuals at high cardiovascular disease risk and had three main findings. First, low-grade inflammation was positively associated with carotid $\beta$-stiffness index and Young's elastic modulus. The latter association was independent of glucose tolerance status, mean arterial pressure and antihypertensive and lipid-lowering medication, current smoking and prior cardiovascular disease. Second, endothelial dysfunction was associated with greater carotid stiffness after adjustment for sex, age, and glucose tolerance status. However, additional 
adjustment for mean arterial pressure and other potential confounders attenuated these associations, which lost statistical significance. Third, the positive association between low-grade inflammation and carotid Young's elastic modulus was primarily driven through a greater carotid diameter.

Thus, low-grade inflammation not only is an important determinant of carotid stiffening in young adults ${ }^{14}$ and middle-aged populations ${ }^{16,17}$, but also in the elderly as suggested by the present results. This study is the first to investigate such an extensive array of biomarkers in relation to carotid artery stiffness in the elderly. We show that each of the individual biomarkers of low-grade inflammation was positively associated with carotid Young's elastic modulus, except for TNF- $\alpha$, with statistically significant associations for SAA, CRP and IL-6 (Figure 9.1). In our study the positive association of low-grade inflammation with carotid Young's elastic modulus was primarily driven through greater diameter (i.e. low-grade inflammation was statistically significantly associated with a greater diameter, but not with distension, pulse pressure or intima-media thickness, i.e. the other variables used to calculate carotid Young's elastic modulus). Additional analyses showed that each of the individual biomarkers of low-grade inflammation was positively associated with carotid diameter, except for TNF- $\alpha$ and SAA, with statistically significant associations for CRP and IL-6. Like the low-grade inflammation overall Z-score, none of the individual biomarkers were associated with distension, local pulse pressure or intimamedia thickness, except for an inverse association between SAA and intima-media thickness. This inverse association is unexpected and we cannot exclude chance. Previous studies in the elderly showed either no associations between SAA and intima-media thickness ${ }^{28,29}$, or a positive association with progression of carotid lesions. ${ }^{30}$ These mixed results suggest that the role for SAA with regard to the carotid artery is complex and needs further study. Nevertheless, taken together these results support the concept that low-grade inflammation is involved in carotid artery remodelling (via a diameter increase) resulting in greater Young's elastic modulus, possibly through effects on the proliferation of smooth muscle cells, the influx of leucocytes and the production of (other) pro-inflammatory substances and chemoattractants in the arterial wall and its matrix. ${ }^{8,31}$ Indeed, a previous study in elderly individuals has shown that a greater carotid diameter was associated with increased mortality. $^{32}$

9 The endothelium has many functions and is itself heterogeneous. ${ }^{33,34}$ The concept of endothelial dysfunction therefore has many dimensions. ${ }^{35}$ FMD is widely believed to reflect endothelium-dependent and largely nitric-oxide-mediated vasodilation and is considered an important functional estimate of endothelial function. ${ }^{35,36}$ Circulating markers, such as vWf, sVCAM-1, sE-selectin, sTM and sICAM-1, 1 ,27,37, are synthesized by endothelial cells. From a conceptual point of view, it is therefore plausible to assume that a smaller FMD and higher circulating concentrations reflect dysfunction of the endothelium and that a combination of these markers gives a valid overall estimate of endothelial dysfunction. This is further illustrated by the fact that both a 
smaller FMD response and higher circulating concentrations of endothelial-derived biomarkers are associated with (incident) cardiovascular disease. ${ }^{2-7}$ Yet, if we repeated the analyses leaving FMD out of the endothelial dysfunction overall Z-score the results were not materially altered. Thus, endothelial dysfunction either estimated by circulating biomarkers, or by circulating biomarkers plus FMD, was not associated with carotid arterial stiffening or remodelling in the elderly. This, however, does not exclude that endothelial dysfunction may play a more prominent role in carotid arterial stiffening at an earlier time-point. ${ }^{38}$

Endothelial dysfunction is closely linked to low-grade inflammation and these concepts are difficult to separate. ${ }^{9,31}$ We used two distinct biomarker scores and show, by mutual adjustment, that the low-grade inflammation, but not the endothelial dysfunction, overall Z-score was associated with greater carotid stiffness. These results therefore suggest that low-grade inflammation and endothelial dysfunction affect carotid stiffness differentially. This is in line with earlier studies $^{14,16,17}$, which also suggested that low-grade inflammation, but not endothelial dysfunction, is an important determinant of carotid arterial stiffening.

By study design, we included extra individuals with type 2 diabetes mellitus. ${ }^{18-20}$ On the one hand, as type 2 diabetes mellitus has been associated with biomarkers of endothelial dysfunction and low-grade inflammation ${ }^{3}$ and with greater carotid stiffness $^{20}$, this might have confounded our results. For this reason, we have adjusted our results for glucose tolerance status, which showed attenuation for each of the reported associations between low-grade inflammation or endothelial dysfunction and the carotid stiffness estimates. On the other hand, it is plausible that diabetes mellitus can lead to increased carotid stiffening through an effect on endothelial dysfunction or low-grade inflammation. If so, then the adjustment for glucose tolerance status can be considered an overadjustment. Thus, the adjusted results presented may be considered conservative.

Additional analyses of the association between low-grade inflammation and carotid Young's elastic modulus showed that this association was stronger in individuals with type 2 diabetes mellitus as compared with normal glucose tolerance and impaired glucose tolerance. Thus, in our study, low-grade inflammation has a stronger effect on arterial stiffening in individuals with type 2 diabetes mellitus than in those without diabetes mellitus. We speculate that a combined action of low-grade inflammation with non-enzymatic glycation of proteins might be responsible for this effect, but this obviously needs further study.

The present investigation had some limitations. First, the biomarkers of endothelial dysfunction and low-grade inflammation were assessed on one occasion only and intrinsic to an overall Z-score is the assumption that each biomarker in the Z-score carries similar weight. Both of these limitations may have caused us to underestimate the reported associations, the former due to error because of bio-variability, and the latter due to error because of a mathematical approach that might not optimally reflect pathophysiology. Second, we can not fully exclude that a long period of frozen 
storage might have effected the absolute biomarker concentration in the stored samples. If so, this would increase random error and thus might have caused us to underestimate the reported associations. Yet, we were able to show in an earlier study that concentrations of sICAM-1, sVCAM-1, CRP and sE-selectin remained stable over a relatively short period ${ }^{39}$ and others have shown that, for example, CRP concentrations remain stable even over a 3 -year period of storage. ${ }^{40}$ Third, our work is in line with previous studies, which showed that low-grade inflammation is a determinant of carotid arterial stiffness, both in healthy adults ${ }^{14}$ and middle-aged populations. ${ }^{16,17}$ The present work however is cross-sectional in nature and therefore inferences about causality should be made with caution. The results nevertheless suggest that low-grade inflammation also plays a role in arterial stiffness and remodelling in the elderly. It is currently unknown however whether the impact of low-grade inflammation on the athero-arteriosclerotic process in the elderly is similar to that in younger individuals [i.e. whether the impact of low-grade inflammation differs over the natural course of athero-/arteriosclerosis (e.g. in the fatty streak vs. the full blown plaque)]. Finally, the present investigation cannot exclude that a survival bias might have affected our results; that is, that the individuals who died before the start of the present study were those with the strongest association between low-grade inflammation or endothelial dysfunction and carotid stiffness. However, such a bias would also have led to an underestimation of the reported associations.

In conclusion, we show that, in the elderly, low-grade inflammation is positively associated with carotid stiffness and that this association was primarily driven through greater diameter. These data suggest that low-grade inflammation, in elderly, plays an important role in carotid artery remodelling and stiffening. 


\section{References}

1. Becker A, van Hinsbergh VW, Jager A, Kostense PJ, Dekker JM, Nijpels G, Heine RJ, Bouter LM, Stehouwer $\mathrm{CD}$. Why is soluble intercellular adhesion molecule-1 related to cardiovascular mortality? Eur J Clin Invest 2002; 32:1-8.

2. Blankenberg S, Rupprecht HJ, Bickel C, Peetz D, Hafner G, Tiret L, Meyer J. Circulating cell adhesion molecules and death in patients with coronary artery disease. Circulation 2001; 104:1336-1342.

3. de Jager J, Dekker JM, Kooy A, Kostense PJ, Nijpels G, Heine RJ, Bouter LM, Stehouwer CD. Endothelial dysfunction and low-grade inflammation explain much of the excess cardiovascular mortality in individuals with type 2 diabetes: the Hoorn Study. Arterioscler Thromb Vasc Biol 2006; 26:1086-1093.

4. Jager A, van Hinsbergh VW, Kostense PJ, Emeis JJ, Yudkin JS, Nijpels G, Dekker JM, Heine RJ, Bouter LM, Stehouwer CD. von Willebrand factor, C-reactive protein, and 5-year mortality in diabetic and nondiabetic subjects: the Hoorn Study. Arterioscler Thromb Vasc Biol 1999; 19:3071-3078.

5. Morange PE, Simon C, Alessi MC, Luc G, Arveiler D, Ferrieres J, Amouyel P, Evans A, Ducimetiere P, Juhan-Vague I; PRIME Study Group. Endothelial cell markers and the risk of coronary heart disease: the Prospective Epidemiological Study of Myocardial Infarction (PRIME) study. Circulation 2004; 109:1343-1348.

6. Ridker PM, Hennekens $\mathrm{CH}$, Buring JE, Rifai N. C-reactive protein and other markers of inflammation in the prediction of cardiovascular disease in women. N Engl J Med 2000; 342:836-843.

7. Yeboah J, Crouse JR, Hsu FC, Burke GL, Herrington DM. Brachial flow-mediated dilation predicts incident cardiovascular events in older adults: the Cardiovascular Health Study. Circulation 2007; 115:2390-2397.

8. Ross R. Atherosclerosis--an inflammatory disease. N Engl J Med 1999; 340:115-126.

9. Borissoff JI, Spronk HM, ten Cate H. The hemostatic system as a modulator of atherosclerosis. N Engl J Med 2011; 364:1746-1760.

10. Schnabel R, Larson MG, Dupuis J, Lunetta KL, Lipinska I, Meigs JB, Yin X, Rong J, Vita JA, Newton-Cheh C, Levy D, Keaney JF Jr, Vasan RS, Mitchell GF, Benjamin EJ. Relations of inflammatory biomarkers and common genetic variants with arterial stiffness and wave reflection. Hypertension 2008; 51:16511657.

11. Lieb W, Larson MG, Benjamin EJ, Yin X, Tofler GH, Selhub J, Jacques PF, Wang TJ, Vita JA, Levy D, Vasan RS, Mitchell GF. Multimarker approach to evaluate correlates of vascular stiffness: the Framingham Heart Study. Circulation 2009; 119:37-43.

12. Yasmin, McEniery CM, Wallace S, Mackenzie IS, Cockcroft JR, Wilkinson IB. C-reactive protein is associated with arterial stiffness in apparently healthy individuals. Arterioscler Thromb Vasc Biol 2004; 24:969-974.

13. Amar J, Ruidavets JB, Bal Dit Sollier C, Bongard V, Boccalon H, Chamontin B, Drouet L, Ferrières J. Soluble CD14 and aortic stiffness in a population-based study. J Hypertens 2003; 21:1869-1877.

14. van Bussel BC, Schouten F, Henry RM, Schalkwijk CG, de Boer MR, Ferreira I, Smulders YM, Twisk JW, Stehouwer CD. Endothelial dysfunction and low-grade inflammation are associated with greater arterial stiffness over a 6-year period. Hypertension 2011; 58:588-595.

15. Mattace-Raso FU, van der Cammen TJ, van der Meer IM, Schalekamp MA, Asmar R, Hofman A, Witteman JC. C-reactive protein and arterial stiffness in older adults: the Rotterdam Study. Atherosclerosis 2004; 176:111-116.

16. Juonala M, Jarvisalo MJ, Maki-Torkko N, Kahonen M, Viikari JS, Raitakari OT. Risk factors identified in childhood and decreased carotid artery elasticity in adulthood: the Cardiovascular Risk in Young Finns Study. Circulation 2005; 112:1486-1493.

17. Eklund C, Kivimaki M, Islam MS, Juonala M, Kahonen M, Marniemi J, Lehtimäki T, Viikari J, Raitakari OT, Hurme M. C-reactive protein genetics is associated with carotid artery compliance in men in The Cardiovascular Risk in Young Finns Study. Atherosclerosis 2008; 196:841-848.

18. Mooy JM, Grootenhuis PA, de Vries H, Valkenburg HA, Bouter LM, Kostense PJ, Heine RJ. Prevalence and determinants of glucose intolerance in a Dutch caucasian population. The Hoorn Study. Diabetes Care 1995; 18:1270-1273. 
19. Spijkerman AM, Adriaanse MC, Dekker JM, Nijpels G, Stehouwer CD, Bouter LM, Heine RJ. Diabetic patients detected by population-based stepwise screening already have a diabetic cardiovascular risk profile. Diabetes Care 2002; 25:1784-1789.

20. Henry RM, Kostense PJ, Spijkerman AM, Dekker JM, Nijpels G, Heine RJ,Kamp O, Westerhof N, Bouter LM, Stehouwer CD . Arterial stiffness increases with deteriorating glucose tolerance status: the Hoorn Study. Circulation 2003; 107:2089-2095.

21. de Valk-de Roo GW, Stehouwer CD, Lambert J, Schalkwijk CG, van der Mooren MJ, Kluft C, Netelenbos C. Plasma homocysteine is weakly correlated with plasma endothelin and von Willebrand factor but not with endothelium-dependent vasodilatation in healthy postmenopausal women. Clin Chem 1999; 45:1200-1205.

22. Henry RM, Ferreira I, Kostense PJ, Dekker JM, Nijpels G, Heine RJ, Kamp O, Bouter LM, Stehouwer CD. Type 2 diabetes is associated with impaired endothelium-dependent, flow-mediated dilation, but impaired glucose metabolism is not; The Hoorn Study. Atherosclerosis 2004; 174:49-56.

23. Laurent S, Cockcroft J, Van Bortel L, Boutouyrie P, Giannattasio C, Hayoz D, Pannier B, Vlachopoulos C, Wilkinson I, Struijker-Boudier H; European Network for Non-invasive Investigation of Large Arteries. Expert consensus document on arterial stiffness: methodological issues and clinical applications. Eur Heart J 2006; 27:2588-2605.

24. Van Bortel LM, Balkestein EJ, van der Heijden-Spek JJ, Vanmolkot FH, Staessen JA, Kragten JA, Vredeveld JW, Safar ME, Struijker Boudier HA, Hoeks AP. Non-invasive assessment of local arterial pulse pressure: comparison of applanation tonometry and echo-tracking. J Hypertens 2001; 19:10371044.

25. Levey AS, Coresh J, Greene T, Marsh J, Stevens LA, Kusek JW, Van Lente F. Expressing the Modification of Diet in Renal Disease Study equation for estimating glomerular filtration rate with standardized serum creatinine values. Clin Chem 2007; 53:766-772.

26. Yudkin JS, Stehouwer CD, Emeis JJ, Coppack SW. C-reactive protein in healthy subjects: associations with obesity, insulin resistance, and endothelial dysfunction: a potential role for cytokines originating from adipose tissue? Arterioscler Thromb Vasc Biol 1999; 19:972-978.

27. Schram MT, Stehouwer CD. Endothelial dysfunction, cellular adhesion molecules and the metabolic syndrome. Horm Metab Res 2005; 37 Suppl 1:49-55.

28. Wohlin M, Helmersson J, Sundstrom J, Arnlov J, Vessby B, Larsson A, Andrén B, Lind L, Basu S. Both cyclooxygenase- and cytokine-mediated inflammation are associated with carotid intima-media thickness. Cytokine 2007; 38:130-136.

29. Leinonen ES, Hiukka A, Hurt-Camejo E, Wiklund O, Sarna SS, Mattson Hulten L, Westerbacka J, Salonen RM, Salonen JT, Taskinen MR. Low-grade inflammation, endothelial activation and carotid intima-media thickness in type 2 diabetes. J Intern Med 2004; 256:119-127.

30. Schillinger M, Exner M, Mlekusch W, Sabeti S, Amighi J, Nikowitsch R, Timmel E, Kickinger B, Minar C, Pones M, Lalouschek W, Rumpold H, Maurer G, Wagner O, Minar E. Inflammation and Carotid Artery-Risk for Atherosclerosis Study (ICARAS). Circulation 2005; 111:2203-2209.

31. Stehouwer CD, Henry RM, Ferreira I. Arterial stiffness in diabetes and the metabolic syndrome: a pathway to cardiovascular disease. Diabetologia 2008; 51:527-539.

32. van Dijk RA, Dekker JM, Nijpels G, Heine RJ, Bouter LM, Stehouwer CD. Brachial artery pulse pressure and common carotid artery diameter: mutually independent associations with mortality in subjects with a recent history of impaired glucose tolerance. Eur J Clin Invest 2001; 31:756-763.

33. Aird WC. Phenotypic heterogeneity of the endothelium: I. Structure, function, and mechanisms. Circ Res 2007; 100:158-173.

34. Aird WC. Phenotypic heterogeneity of the endothelium: II. Representative vascular beds. Circ Res 2007; 100:174-190.

35. Deanfield J, Donald A, Ferri C, Giannattasio C, Halcox J, Halligan S, Lerman A, Mancia G, Oliver JJ, Pessina AC, Rizzoni D, Rossi GP, Salvetti A, Schiffrin EL, Taddei S, Webb DJ; Working Group on Endothelin and Endothelial Factors of the European Society of Hypertension. Endothelial function and dysfunction. Part I: Methodological issues for assessment in the different vascular beds: a statement by the Working Group on Endothelin and Endothelial Factors of the European Society of Hypertension. J Hypertens 2005; 23:7-17. 
36. Thijssen DH, Black MA, Pyke KE, Padilla J, Atkinson G, Harris RA, Parker B, Widlansky ME, Tschakovsky $M E$, Green DJ. Assessment of flow mediated dilation (FMD) in humans: a methodological and technical guideline. Am J Physiol Heart Circ Physiol 2011; 300:H2-12.

37. Vischer UM. von Willebrand factor, endothelial dysfunction, and cardiovascular disease. J Thromb Haemost 2006; 4:1186-1193.

38. McEniery CM, Wallace S, Mackenzie IS, McDonnell B, Yasmin, Newby DE, Cockcroft JR, Wilkinson IB. Endothelial function is associated with pulse pressure, pulse wave velocity, and augmentation index in healthy humans. Hypertension 2006; 48:602-608.

39. Hartweg J, Gunter M, Perera R, Farmer A, Cull C, Schalkwijk C, Kok A, Twaalfhoven H, Holman R, Neil A. Stability of soluble adhesion molecules, selectins, and C-reactive protein at various temperatures: implications for epidemiological and large-scale clinical studies. Clin Chem 2007; 53:1858-1860.

40. Hutchinson WL, Koenig W, Frohlich M, Sund M, Lowe GD, Pepys MB. Immunoradiometric assay of circulating C-reactive protein: age-related values in the adult general population. Clin Chem 2000; 46:934-938. 


\section{Chapter 10}

Summary and general discussion 
Chapter 10 


\section{Summary and general discussion}

The main aim of this thesis was to investigate whether endothelial dysfunction and low-grade inflammation are determined by diet and cause arterial stiffness. In order to investigate the vascular endothelium as one integrated organ, while taking its heterogeneity into account, the present thesis focused on generalized endothelial dysfunction with the use of a conceptual multi-marker approach, while concurrently addressing the closely related and multi-faceted process of low-grade inflammation (Figure 10.1). ${ }^{1-6}$

First, we hypothesized that consumption of a healthy diet rich in fish, vegetables and fruit, with moderate alcohol-containing beverages and low in dairy products, is associated with less endothelial dysfunction and low-grade inflammation. This hypothesis was investigated at the food group and/or nutrient compound level, in community-dwelling individuals over a wide age range, in health and disease, and using both cross-sectional and longitudinal data, while consistently adjusting for each of the other food groups (or nutrients).

Second, we hypothesized that greater endothelial dysfunction and more low-grade inflammation are associated with greater arterial stiffness. This hypothesis was investigated in community-dwelling individuals over a wide age range, in health and disease, and using both cross-sectional and longitudinal data.

This final chapter, first, summarizes the main findings of this thesis in paragraph 10.1. The most important methodological considerations regarding the studies presented are discussed in paragraph 10.2. Then, the overall results and the public health implications are given in paragraphs 10.3 and 10.4, respectively. Finally, directions for future research are proposed in paragraph 10.5 . 


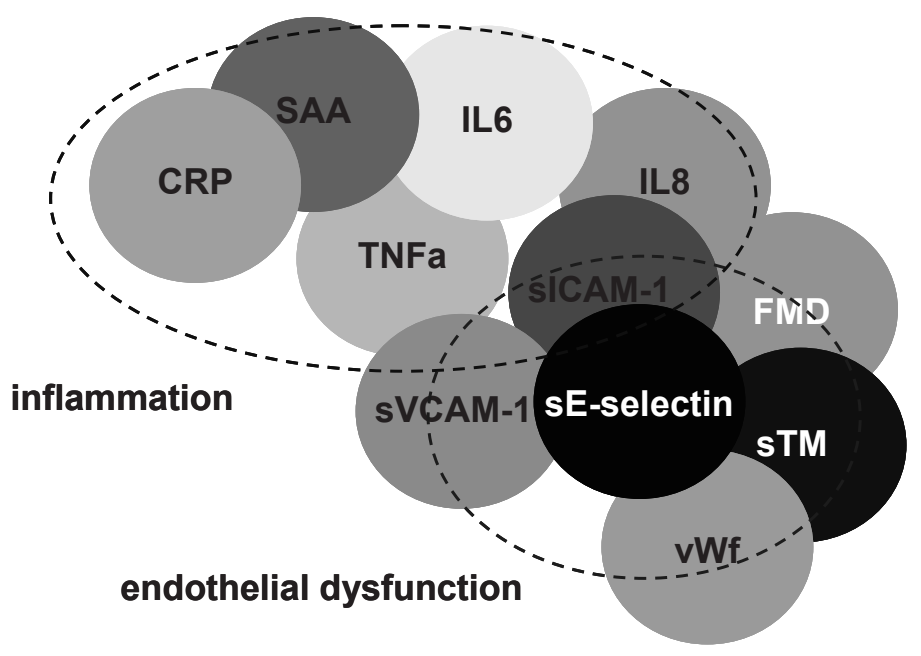

Figure 10.1 Multiple biomarkers of endothelial dysfunction and low-grade inflammation, a conceptual presentation of related biomarkers; the dashed circle represents endothelial dysfunction, whereas the dashed ellipse represents low-grade inflammation.

Combined scores of conceptually related biomarkers were used to investigate endothelial dysfunction and low-grade inflammation over a broad range of domains. This reduces the influence of biological variability and the number of associations that need to be explored. ${ }^{7}$ However, a limitation of these combined scores is the intrinsic assumption that each biomarker in the scores carries similar weight. In fact, it is unknown whether this is the case for each individual biomarker with regard to CVD risk. ${ }^{7-9}$ The chosen approach may therefore not reflect pathophysiology optimally. Based on currently available evidence, ${ }^{9-25}$ it is, however, reasonable to assume that the biomarkers used in this thesis carry similar weight with regard to CVD risk. Nevertheless, when future evidence underscores the importance of a particular biomarker, the combined scores can easily be adapted by incorporating a weight for a particular biomarker.

CRP, C-reactive protein; SAA, serum amyloid A; IL-6, interleukin 6; TNF- $\alpha$, tumour necrosis factor alpha; IL-8, interleukin 8; sICAM-1, soluble intercellular adhesion molecule 1; FMD, flow-mediated dilation; sVCAM-1, soluble vascular cell adhesion molecule 1; sE-selectin, soluble endothelial selectin; sTM, soluble thrombomodulin; vWf, von Willebrand factor.

\section{Summary (10.1)}

In part $\mathbf{1}$ (chapters $\mathbf{2}$ and 3) we, first, presented some methodological considerations with regard to the measurement of endothelial dysfunction and low-grade inflammation. In chapter $\mathbf{2}$ we performed a method comparison study to crossvalidate multiplex technology with well-established single-biomarker methods for biomarker detection to implement the more efficient multiplex technology in ongoing cohort studies. The results showed that absolute concentrations of CRP, SAA, SICAM-1 and SVCAM-1 differed significantly between well-established single-biomarker methods and a multi-array platform of MesoScaleDiscovery (MSD). Equations 
retrieved by weighted Deming regression enabled proper realignment of the data to overcome these absolute differences. The overall pattern of associations between levels of the individual biomarkers with glucose metabolism, weight, metabolic syndrome and smoking status did not differ by method of detection. The multi-array platform, thus, is a potential tool that is attractive for multi-marker measurements and was applied in several cohort studies presented in this thesis.

In chapter 3 we investigated 30 presumed healthy men and showed that the measurement of flow-mediated vasodilation (FMD) is improved by taking the blood flow stimulus (i.e. hyperaemia) into account. Study protocol-induced changes over time in 'new' indices of FMD, which accounted for the flow-stimulus, were more easily detected than changes in the conventional FMD, while the reproducibility of these measures was comparable. Operationalization of the flow-stimulus by the relative peak or relative mean hyperaemic change in blood flow velocity showed similar results. Although both glucose $(n=22)$ and water loads $(n=8)$ were applied, the study design did not permit to differentiate whether the changes over time in FMD ('new' indices or conventional FMD) were determined by repeated measurements (by using the FMD apparatus repeatedly), by volume load (both water and glucose solution) or by a glucose effect (as suggested previously ${ }^{26-31}$ ). This study, thus, illustrates the importance of the evaluation of the flow-stimulus in FMD measurements and the flow-stimulus was taken into account when FMD was investigated in this thesis.

In part 2 (chapters 4-7) we examined the associations between diet (i.e. five food groups in chapters 4-6: fish, fruit, vegetables, alcohol-containing beverages and dairy products; and nutrient compounds in chapter 7) $)^{32-48}$ and comparable, extensive sets of biomarkers of endothelial dysfunction and low-grade inflammation in multiple settings: i.e. in young adults (chapter $\mathbf{4}$ and 7) and older adults (chapters $\mathbf{5}$ and 6), in healthy individuals (chapter 4 ) and individuals with diabetes mellitus (either type 1 or type 2) and/or CVD (chapters 5-7), and using repeated dietary data (chapters 6 and 7) and/or repeated biomarker data (chapters 4 and 6). In chapter 4 we showed that fish consumption, but none of the other food groups, was associated with decreased endothelial dysfunction and low-grade inflammation during 6-year follow-up in 301 young healthy adults of the AGAHLS. The combined nutrient compounds eicosapentaenoic acid and docosapentaenoic acid were associated with decreased endothelial dysfunction and low-grade inflammation and together they explained $83 \%$ of decreased endothelial dysfunction and $40 \%$ of decreased low-grade inflammation associated with higher fish consumption. Importantly these results were independent of each of the other food groups.

In chapter $\mathbf{5}$ we re-investigated the same associations in a cross-sectional study in 801 older adults of the Hoorn Study, which included data on FMD, which was additionally investigated as a measure of endothelial dysfunction. Alcohol and red wine consumption were associated with lower endothelial dysfunction; i.e. both lower circulating biomarkers and a greater FMD. The associations for FMD were, however, 
not statistically significant. None of the food groups showed an association with lowgrade inflammation, except for the consumption of red wine. Red wine consumption was associated with less low-grade inflammation and this association was stronger in participants with diabetes mellitus type 2 and CVD as compared to participants who were free of clinical disease. Importantly, these results were independent of each of the other food groups.

In chapter 6 we investigated, in 557 older adults of the CODAM Study, the associations between, on the one hand, the consumption of fish, fruit, vegetables, alcohol-containing beverages and dairy products and, on the other hand, endothelial dysfunction and low-grade inflammation over a 7-year period. We showed that a higher consumption of fish was associated with less endothelial dysfunction, whereas the consumption of dairy products, fruit, vegetables and alcohol-containing beverages showed no association with endothelial dysfunction. Additional analyses at the food component level showed that a higher consumption of, in particular, lean fish, raw vegetables, and a lower consumption of high-fat dairy products were associated with less endothelial dysfunction. None of the five food groups were associated with lowgrade inflammation. However, additional analyses at the food component level showed that a higher consumption of, in particular, wine was associated with less lowgrade inflammation, although this was borderline statistically significant. Stratified analyses for individuals with clinical disease showed that the consumption of fresh fruit favourably impacted on low-grade inflammation in those with diabetes and/or CVD, whereas the consumption of total and high-fat dairy products impacted unfavourably on low-grade inflammation in those with diabetes and/or CVD. Importantly these results were independent of each of the other food groups.

In chapter 7 we investigated, in 491 individuals with diabetes mellitus type 1 of the EURODIAB Study, the association between a healthy diet and endothelial dysfunction and low-grade inflammation and focused on nutrient compounds. We showed that individuals with greater endothelial dysfunction consumed less fibre (both soluble and insoluble fibre), less polyunsaturated fat and less vegetable protein over the 8-year study period, whereas no associations were found for carbohydrate, (other types of) fat, cholesterol or (animal) protein. In addition, individuals with greater low-grade inflammation consumed less (soluble) fibre, less polyunsaturated fat, and more cholesterol over the 8-year study period, whereas no associations were found for insoluble fibre, carbohydrate, (other types of) fat and (any type of) protein.

Finally, in part $\mathbf{3}$ (chapters $\mathbf{8}$ and 9) we investigated whether extensive sets of biomarkers of endothelial dysfunction and low-grade inflammation were associated with greater arterial stiffness in multiple settings: i.e. in young healthy adults (chapter 8) and elderly individuals with diabetes mellitus type 2 and CVD (chapter 9), and using repeated biomarker and arterial stiffness data (chapter $\mathbf{8}$ ). In chapter $\mathbf{8}$ we showed, in 293 young healthy adults of the AGAHLS, that endothelial dysfunction and low-grade inflammation were associated with greater arterial stiffness over a 6-year 
period. Endothelial dysfunction was associated with greater femoral artery stiffness, whereas low-grade inflammation was associated with both greater carotid and femoral artery stiffness. However, endothelial dysfunction and low-grade inflammation were not associated with stiffness of the carotid-femoral segment. As endothelial dysfunction and low-grade inflammation are closely related ${ }^{49}$ (Figure 10.1), we investigated whether their associations with arterial stiffness were either independent or interdependent by mutual adjustments for each other. The results showed that the associations between, on the one hand, endothelial dysfunction or low-grade inflammation and, on the other hand, femoral artery stiffness were interdependent, whereas the result for low-grade inflammation and carotid artery stiffness was independent. Both the associations of endothelial dysfunction and lowgrade inflammation with greater femoral artery stiffness were primarily driven through decreased distention, whereas the association between low-grade inflammation and greater carotid stiffness was not primarily driven through any of the arterial parameters.

In chapter 9 we re-investigated the associations between, on the one hand, endothelial dysfunction and low-grade inflammation and, on the other hand, carotid artery stiffness in a cross-sectional study in 601 older adults of the Hoorn Study. This study included data on FMD and this measure of endothelial dysfunction was additionally investigated. Although endothelial dysfunction was not associated with greater carotid artery stiffness, the results for low-grade inflammation showed a positive association. The association between low-grade inflammation and greater carotid stiffness was primarily driven through a greater carotid diameter (Figure 10.2).

\section{General discussion (10.2)}

\section{Internal validity}

We investigated throughout the thesis the same hypotheses using different study populations, which might explain differences in some of the results between studies. Results of observational studies, however, might be influenced by the occurrence of selection bias, information bias and confounding ${ }^{50}$ and these phenomena and their potential impact on the presented studies need to be discussed before interpreting the overall result of the present thesis. 


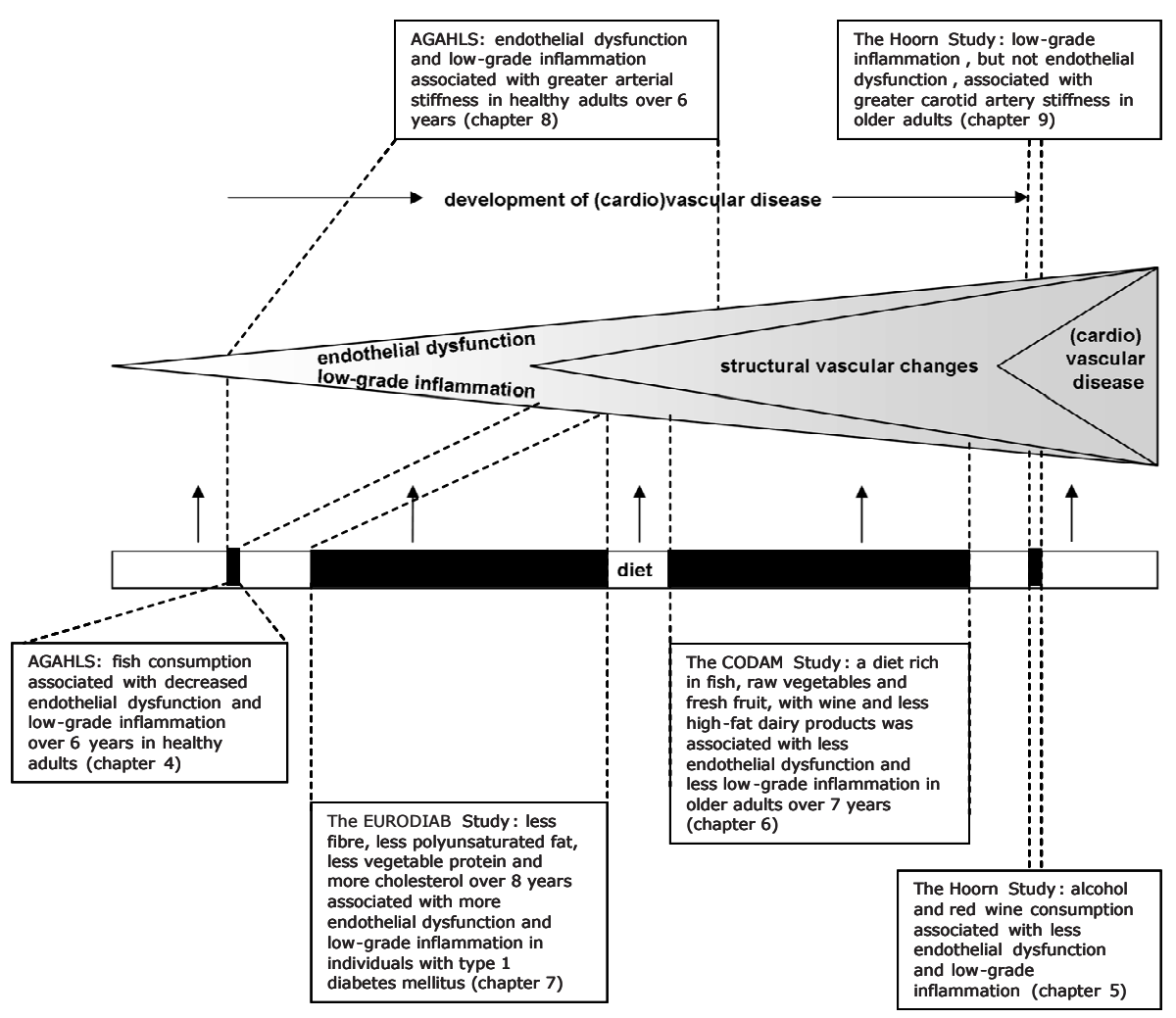

Figure 10.2 Pathophysiological framework for dietary effects on the initiation and progression of cardiovascular disease studied in the present thesis: summary of the main results.

\section{Selection bias}

The selection of study participants may influence associations, resulting in either overor underestimation of the associations under study. ${ }^{50}$ For the studies in the present thesis, we considered selective non-response, survival bias and loss to follow-up to be potential sources of selection bias. Therefore, we undertook several efforts across the different studies to minimize the impact of potential selection bias in the analyses and, additionally, we aimed to interpret the effect of potential selection bias in the reported associations e.g. through comparison of baseline characteristics.

In the EURODIAB and CODAM Studies we used the statistical technique of generalized estimating equations, which maximizes the number of study participants included in the analyses regardless of missing data. ${ }^{57}$ This minimized the influence of missing data and its potential selection bias due to loss to follow-up and selective non-response. However, we recognize that selection bias might still be present. 
When the generalized estimating equations analyses were repeated in all individuals of the AGAHLS regardless of missing data (chapter 8, data not shown), the associations between endothelial dysfunction and low-grade inflammation, and arterial stiffness did not materially change as compared to the results presented for individuals with complete longitudinal data. This supports our assumption that it is highly unlikely that any of the 698 boys and girls, who started in 1976 at age 12 years, refused (at any time-point during the 30-year study period) further participation depending on the reported associations of the 2000 and 2006 follow-up examinations. In addition, bias due to loss to follow-up between 2000 and 2006 was unlikely as the 301 participants, overall, did not differ from 72 drop-outs at 2000, except for smoking behaviour. Therefore, it is unlikely that selection bias has influenced the results of the AGAHLS.

Of the studies in the present thesis, the influence of selection bias is perhaps highest in the Hoorn Study. Selective non-response may have particularly influenced the results for FMD in the Hoorn Study and might explain why results for FMD, in contrast to those for circulating biomarkers, were not statistically significant (chapter 5). Individuals without FMD data had a higher body mass index than participants with FMD data, which may be explained by the fact that FMD-quantification is easily impaired in obese individuals. ${ }^{53}$ Importantly, obesity has been associated with a smaller $\mathrm{FMD}^{54}$, an unhealthy $\operatorname{diet}^{55}$ and greater arterial stiffness. ${ }^{56}$ The reported associations between a healthy diet and greater FMD and between a smaller FMD and greater arterial stiffness in the Hoorn Study might therefore be underestimated. In addition, the Hoorn Study started in 1989 as a population-based cohort study on glucose metabolism and its complications. The studies in the present thesis used cross-sectional data from the 2000-2001 follow-up examination, when diet, biomarkers of endothelial dysfunction and low-grade inflammation, and arterial stiffness were determined. A survival bias may therefore also have resulted in underestimation of the reported associations, as individuals with an unhealthy diet, greater endothelial dysfunction, higher level of inflammation, and greater arterial stiffness were most likely those who dropped out, because these characteristics have been associated with higher CVD mortality. ${ }^{9-12,23,34,35,51,52}$ Based on the clinical characteristics, the Hoorn Study as compared to the other studies, has the highest chance of bias in the reported associations due to such a healthy survivor effect, as this study includes older individuals with a high prevalence of CVD (51.4\%) and diabetes mellitus type 2 (40.6\%). Although the healthy survivor effect cannot be excluded, it most likely has played a less prominent role in the CODAM Study, the EURODIAB Study and, in particular, the AGAHLS, due to the fact that these populations were younger and relatively healthier. In conclusion, both survival bias and selective non-response most likely have led to an underestimation of the results in the Hoorn Study, and potentially might explain the failure to observer some of the associations that were likely based on previous results of other studies. ${ }^{51,52}$ 


\section{Information bias}

Two types of information bias may have influenced the reported associations, i.e. random error and systematic error. Random error may result in underestimation of associations (i.e. bias towards zero) $)^{50,53}$ and can be reduced by more precise and repeated measurements. Systematic error results in either over- or underestimation of the associations under study if measurement error depends on risk factor exposure. ${ }^{50}$ For the studies in the present thesis, both types of bias might have influenced the results.

The strength of the present thesis is the use of repeated measurements of determinants and/or outcome variables that reduced the influence of random error in multiple studies; e.g. repeated dietary assessments were done in the CODAM and EURODIAB Studies; repeated biomarker assessments were done in the AGAHLS and CODAM Studies; repeated FMD parameters were assessed in the study in chapter 3; and repeated arterial stiffness estimates were determined in the AGAHLS. This has increased the power to detect relevant associations/differences. ${ }^{50}$ In particular, the comparison of the results of the Hoorn and CODAM Studies is remarkable. Both studies used the same Food Frequency Questionnaire (FFQ), which was applied twice in the CODAM Study and once in the Hoorn Study. Associations between diet and endothelial dysfunction and low-grade inflammation were clearer in the CODAM Study as compared to the Hoorn Study. This observation is in line with the fact that random error might have diluted associations (i.e. towards zero) in the Hoorn Study, whereas it suggests that dietary assessment was more precise in the CODAM Study.

\section{Diet}

With regard to dietary exposure assessment, three methods were employed and each of these methods has repeatedly and successfully been used in large populationbased studies. ${ }^{54,55}$ The dietary history method was used in the AGAHLS, ${ }^{56-60}$ the same FFQ was used in both the Hoorn and CODAM studies ${ }^{54,55,61-65}$ and the food record method was used in the EURODIAB Study. ${ }^{66-69}$ For each of those methods a reasonable to good reproducibility has been reported. ${ }^{54-69}$ However, although each method has its advantages over the others (as described in the introduction of this thesis) and this might explain some of the differences between results of the studies, several results were consistently found and this was independent of the dietary assessment method used. For example, favourable associations for fish consumption with endothelial dysfunction were found for both the diet history method (chapter 4) and the FFQ (chapter 6); and favourable associations for fibre and vegetable protein consumption with endothelial dysfunction, and for raw vegetable consumption with endothelial dysfunction were found for both the food record (chapter 7) and the FFQ (chapter 6), respectively.

Systematic dietary measurement error however, might have influenced the results in each of the studies, as it is has been shown that persons with a high body mass index 
systematically underestimate (unhealthy) food intake compared to persons with normal weight. ${ }^{54,55}$ Because obesity has been associated with an unhealthy diet ${ }^{70}$ and with worse endothelial dysfunction and low-grade inflammation ${ }^{7,71}$, this systematic underestimation of (unhealthy) food intake results in an underestimation of the association between a healthy diet and less endothelial dysfunction and low-grade inflammation. In order to minimize the effect of systematic dietary measurement error, we have adjusted the results for energy intake and other variables that have been shown to influence reported dietary intake (e.g. body mass index). ${ }^{54,55}$ In conclusion, a reasonable to good reproducibility has been reported for each of the methods of dietary assessment, although precise and valid assessment remains an important issue in nutritional epidemiology. ${ }^{54-69}$

\section{Circulating biomarkers}

Circulating biomarkers were measured in duplicate and averaged. This enhances the precision of measured concentrations. In our studies, the reproducibility of circulating biomarker measurements was good, with the majority of intra- and inter-assay coefficients of variation $<10 \%$. In addition, several circulating biomarkers that were measured twice in the CODAM Study, by 'old' and 'new' methods, were averaged after appropriate re-alignment (chapters $\mathbf{2}$ and 6). To further reduce the influence of random measurement error in the longitudinal studies both baseline and follow-up samples were measured in pairs within one assay. Finally, biomarkers were combined into overall scores aiming to reduce the influence of biological variability (Figure 10.1). ${ }^{7}$ Additionally, this approach limits the number of associations that require statistical exploration and thus reduces the chance of a type 1 error. ${ }^{50}$ In conclusion, the influence of random error was minimized by multiple measurements and biological variability was reduced by combination of multiple biomarkers into scores.

Sources of potential systematic error that may bias associations ${ }^{72}$ include different methods of detection, the lack of standardization of commercial kits, lot-to-lot variation within biomarker assays, different sample matrices, and different storage times. With regard to the latter, we, however, have previously shown that concentrations of CRP, sICAM-1, SVCAM-1 and sE-selection remained stable over a relatively short period of time ${ }^{73}$ and others have confirmed stable concentrations of CRP after a 3-year storage time. ${ }^{74}$ Overall, we have minimized the effect of systematic measurement error by using one biomarker detection method per study, within one lot of assays, and aiming to use the same sample matrix for repeated measurements. This approach conserved ranking of individuals within the populations studied. Importantly, we showed in detail (chapter 2) that absolute differences in biomarker levels can be overcome by appropriate realignment of the data and that the validity of associations remains if ranking is unaffected. In conclusion, systematic measurement error most likely did not threaten the validity of the reported associations as ranking was not impaired. 


\section{Flow-mediated dilation (FMD)}

FMD measurements including flow-stimulus and diameter variables were measured continuously in the study in chapter 3. Continuous measurement allows to estimate the extent of the flow and diameter changes precisely ${ }^{75}$, thereby reducing the influence of random error ${ }^{50,76}$, which is known to impair FMD measurements. ${ }^{77-81}$ In contrast, in the Hoorn Study, the flow and diameter variables were measured intermittently, due to technical limitations of ultrasound equipment at the time when these measurements were done. ${ }^{71}$ In fact, the FMD protocol in the Hoorn Study included after cuff-release one measurement of the flow-stimulus (i.e. 15 seconds) and four measurements of the brachial artery diameter (i.e. at 45, 90, 180 and 300 seconds), whereas these variables were continuously measured for 5 minutes in the study in chapter $\mathbf{3}$. The maximum diameter response at any of the intermittent time-points was considered as the maximal diameter response, whereas the continuous measurement allowed reconstructing the diameter curve throughout time to determine the maximal diameter response. Nevertheless, the Hoorn Study protocol was done according to international guidelines that are currently approved ${ }^{76,82}$, and is considered an improvement compared to the single diameter determination at 60 seconds after cuff-release, originally proposed by Celermajer et al.. ${ }^{83}$ Previous studies indeed have shown that the maximum change in diameter with intermittent measurements is lower and decreases further according to a smaller number of timepoints assessed, whereas continuous measurement increases the precision. ${ }^{76}$ As a result, FMD in the Hoorn Study might thus have been underestimated compared to currently available continuous flow-stimulus and diameter parameters. ${ }^{75}$ This might have resulted in underestimation of the reported associations for FMD in the Hoorn Study. In the study in chapter $\mathbf{3}$ we, like others, ${ }^{84}$ have further appreciated several important issues with regard to FMD-quantification. We showed that the flowstimulus needs to be taken into account. In the Hoorn Study, we therefore, have adjusted the associations between diet and FMD for the flow-stimulus. Given the abovementioned considerations, the results for FMD in the Hoorn Study should be interpreted with caution.

\section{Arterial stiffness}

Arterial stiffness was measured according to international guidelines. ${ }^{85}$ Reproducibility was reasonable to good as the within- and between observer coefficients of variation for the carotid artery stiffness parameters were $<10 \%$ and for the femoral and brachial artery stiffness variables were $<15 \% .{ }^{86-88}$ Pulse pressure and all arterial stiffness estimates decreased between 2000 and 2006 at the population level in the AGAHLS. This "unexpected" decrease of pressure might be explained by the use of two different blood pressure devices. Alternatively, the decrease may be real as diastolic blood pressure increased more steeply over the course of life within the AGAHLS than systolic blood pressure and increases in pulse 
pressure with ageing are often observed after the $5^{\text {th }}$ or $6^{\text {th }}$ decades of life as a consequence of arterial stiffening. ${ }^{89}$ Nevertheless, a systematic underestimation of arterial stiffness at 2006 does not materially change the reported associations as ranking is unaffected.

\section{Confounding}

Confounding may bias associations in either direction. ${ }^{50}$ Regression techniques were used throughout the thesis to minimize the influence of confounding in the reported associations. Overall, each of the study populations were extensively characterized and that enabled to appropriately address the potential influence of confounding, although bias due to residual confounding can never be excluded in observational studies. $^{50}$

Previous studies on the associations between components of a healthy diet and endothelial dysfunction and low-grade inflammation were often poorly adjusted for other food groups or nutrient compounds. ${ }^{90-93}$ Therefore, it remains unclear which food groups are independent determinants of endothelial dysfunction and low-grade inflammation. In addition, food consumption is closely related to other lifestyle factors such as physical activity, smoking behaviour, alcohol consumption and body mass index ${ }^{54}$, and together with hypertension, renal dysfunction, and the presence of diabetes mellitus and cardiovascular disease, these lifestyle factors might confound associations between diet and endothelial dysfunction and low-grade inflammation and were therefore taken into account in the analyses. Educational level might also be a confounder, but was not available in the AGAHLS and CODAM Studies. Potential confounding by educational level in the AGAHLS is, however, considered small as the participants, by design, were class-mates at secondary school. In the present thesis we, thus, have aimed to adjust for each of the abovementioned potential confounders, and in particular, for fish, vegetable, fruit, alcohol-containing beverages and/or dairy products in the associations between diet and endothelial dysfunction and low-grade inflammation.

In the EURODIAB Study, we investigated nutrient compounds, instead of food groups. We used the nutrient residual method to adjust the associations for energy intake because total energy intake might act as a confounder for several reasons. Total energy intake may be a determinant of endothelial dysfunction and low-grade inflammation. In addition, total energy intake and consumption of most nutrients are positively correlated and absolute intake of a nutrient is therefore unrelated to diet composition. $^{54}$ Energy-adjusted nutrient residuals were, therefore, used as determinants. Furthermore, if energy intake is correlated to other lifestyle variables considered in the multivariate models, it might still act as a confounder and the associations between energy-adjusted nutrient residuals and endothelial dysfunction and low-grade inflammation were therefore additionally adjusted for energy intake. ${ }^{54}$ 
Re-analyses with absolute nutrient intakes, however, showed comparable results in the EURODIAB Study.

Endothelial dysfunction and low-grade inflammation are closely linked and difficult to separate conceptually (Figure 10.1). ${ }^{49}$ In contrast to others, ${ }^{94-110}$ we used mutual adjustments for endothelial dysfunction and low-grade inflammation to show that the associations between endothelial dysfunction and/or low-grade inflammation, and femoral artery stiffness were interdependent, while the results for low-grade inflammation and carotid artery stiffness were independent. The latter result was present in young adults and elderly individuals (chapters 8 and 9).

\section{External validity}

The major strength of the present thesis is that it addresses the hypotheses in multiple settings: i.e. in community-dwelling individuals over a wide age range, and in health and disease. However, populations consisted mainly of Caucasians. The use of multiple settings contributes to the external validity, which means that the results may be extrapolated beyond the specific study populations, and thus, can be generalized to other Caucasian populations with a certain degree of confidence.

\section{Longitudinal study designs and causality}

Longitudinal studies, as compared to cross-sectional data, may provide some more insight into causality as temporality within a relationship favours causality. ${ }^{50,111}$ The present results showed that longitudinal associations were primarily determined by between-subject associations, and not by within-subject associations. It is important to note, however, that within-subject associations are more easily influenced by measurement error as changes over time in general are relatively small. ${ }^{112}$ On the one hand, the longitudinal results in our studies imply that associations might already have existed and did not change over the studied period, which argues against causality. On the other hand, the associations between exposure and outcome remained (chapters 6 and 8 ), suggesting that the exposure continues its effect on the outcome throughout the longitudinal follow-up period, which favours causality. The longitudinal results in our studies, in particular, confirm the presumption that associations between diet and endothelial dysfunction and low-grade inflammation from cross-sectional studies may be extrapolated over time, as was suggested by the observation that diet remains stable over time. ${ }^{113}$ In addition, we provide evidence that the associations between endothelial dysfunction and low-grade inflammation, and arterial stiffness are stable over time in young adults (chapter 8 ). In conclusion, longitudinal designs provide more insight into the underlying associations. Nevertheless, it is important to note that the studies in the present thesis are observational and that even longitudinal designs cannot prove causality by themselves. 
In the EURODIAB Study, we investigated the hypotheses whether healthy nutrient consumption was associated with less endothelial dysfunction and less low-grade inflammation. Nutrient consumption was assessed at baseline and at follow-up, and biomarkers were measured at follow-up only. Dietary data, however, were missing in a considerable part of the population at follow-up. To overcome this limitation we chose to use generalized estimating equations to model the data. ${ }^{112}$ Our models, in practice, were, therefore, reversed and in fact answered a retrospective kind of research question: i.e. to what extent did nutrient intake over the 8-year period differ according to endothelial dysfunction and low-grade inflammation at the end of this period? This approach has the advantage to include all available dietary exposure data, which was prospectively obtained, regardless of missing data. However, it does not have the advantages of a truly longitudinal design. ${ }^{112}$ For example, reversed causality might have played a role in the EURODIAB Study, as study participants with greater endothelial dysfunction and low-grade inflammation at the end of the 8-year study period might also be those with greater endothelial dysfunction and low-grade inflammation at earlier time-points, and consequently might have been more prone to consume an unfavourable diet. However, we consider this scenario unlikely as individuals are unaware of their endothelial dysfunction and low-grade inflammation as both traits have an asymptomatic character.

\section{Overall interpretation of the results (10.3)}

After extensive considerations of the most important methodological issues, we conclude that the present thesis provides evidence that a habitual healthy diet, rich in fish, vegetables and fruit, with moderate alcohol-containing beverages and preferably (red) wine, and with low- instead of high-fat dairy products may reduce CVD through favourably targeting endothelial dysfunction and low-grade inflammation. It expands previous evidence on the relationship between diet and endothelial dysfunction and low-grade inflammation ${ }^{90-93,114-120}$ that was inconsistent due to different sets of biomarkers $^{90-93,114-120}$; the use of multiple ${ }^{91-93,114-120}$ versus single biomarkers ${ }^{90}$; the inclusion of either endothelial dysfunction or low-grade inflammation ${ }^{90}$; the use of either diet scores ${ }^{114-116}$ or individual food components ${ }^{90-93,117-120}$; the often poorly mutual adjustment for other food groups ${ }^{90-93}$; and the investigation of primarily crosssectional data ${ }^{90-93,114-120}$.

The exact underlying mechanisms how diet influences endothelial dysfunction and/or low-grade inflammation remain unclear, although several mechanisms are thought to be involved: modulation of cell membrane stability; inhibition of intracellular signaling pathways that activate the inflammatory response; reduction of oxidative stress; modulation of endothelial nitric oxide synthase (eNOS) activity through the alteration of $\mathrm{Ca}^{2+}$ channels, of the number of caveoli, and of transcriptional and posttranslational modifications of eNOS; modulation of the eicosanoid pathway by 
alteration of prostaglandin synthesis; and modulation of endothelial-derived hyperpolarizing factor, which may influence vascular tone via the alteration of $\mathrm{Ca}^{2+}$-activated $\mathrm{K}^{+}$channels in vascular smooth muscle cells. ${ }^{38-44,47}$ Taken together, these mechanisms ${ }^{38-44,47}$ and our results provide evidence for a pathophysiological framework how diet may influence endothelial dysfunction and low-grade inflammation and thereby the development of CVD.

The results with regard to arterial stiffness suggest that endothelial dysfunction and low-grade inflammation may cause arterial stiffening. It expands previous evidence that was cross-sectional ${ }^{94-110}$, focused on either endothelial dysfunction ${ }^{108}$ or lowgrade inflammation ${ }^{94,98-102,104,105,107,109-110}$, and studied few biomarkers. ${ }^{94-102,104,105,107-110}$ In addition, we showed for the first time that endothelial dysfunction and low-grade inflammation, at least partially, were associated with greater arterial stiffness in an interdependent way. Endothelial dysfunction and low-grade inflammation may lead to arterial stiffening through increased functional stiffening, due to an increase in vascular smooth muscle cell tone through reduced nitric-oxide availability and increased activity of vasoconstrictors such as endothelin-1. ${ }^{85,121-124}$ In addition, they may lead to increased structural stiffening, due to smooth muscle cell proliferation and a shift from elastin towards relatively more collagen synthesis, and deposition of calcium on elastin. ${ }^{85,121,122}$ In conclusion, our results, with these mechanisms, support the hypothesis that endothelial dysfunction and low-grade inflammation cause arterial stiffening.

\section{Public health implications (10.4)}

Diet is perhaps the most modifiable CVD risk factor. ${ }^{125}$ Endothelial dysfunction and low-grade inflammation are important players in the development and progression of CVD. ${ }^{1-6,9-24}$ The present thesis provides evidence that a habitual healthy diet may reduce CVD through favourably targeting endothelial dysfunction and low-grade inflammation and this impacts scientifically, clinically and on the population level.

From a scientific point of view, the population-based studies in this thesis are important because they provide insight into the underlying mechanisms how dietary habits of community-dwelling individuals have their effects on development and progression of CVD. Our results are complementary to controlled laboratory experiments necessary to increase our understanding of specific (intra-)cellular pathways involved in endothelial dysfunction and low-grade inflammation ${ }^{1,2}$ and more importantly allow to translate the impact of research on endothelial dysfunction and low-grade inflammation towards the general population. This thesis, thereby, contributes to the translation of concepts studied in basic science to the implementation of targets for relevant lifestyle interventions in the general population that contribute to a reduction in overall CVD morbidity and mortality. ${ }^{1-6,9-24}$ 
From a clinical point of view, the circulating biomarkers of endothelial dysfunction, FMD, the circulating biomarkers of low-grade inflammation and arterial stiffness estimates studied do currently not yet fulfill a role. ${ }^{126,127}$ One of the reasons is that these variables do not yet improve current prediction rules. ${ }^{126-128}$ In addition, to monitor disease development in the clinic by biomarkers these should have the ability to change over time, the ability to be affected by interventions over time, and such changes in biomarkers over time should be related to change in CVD risk. ${ }^{129}$ However, the improvement of biomarkers of endothelial dysfunction, low-grade inflammation and arterial stiffness is not yet a sufficiently valid substitute for improvement of cardiovascular prognosis. ${ }^{130}$ The results of this thesis, nevertheless, should encourage physicians to advise their patients on a healthy lifestyle and in particular a habitual healthy diet. The additional stratified analyses in the Hoorn and CODAM Studies, with the results of the EURODIAB Study, suggest that implementation of a healthy diet may be most important in individuals with existing clinical diseases, such as diabetes mellitus type 1 and type 2, and/or CVD. This supports the view that dietary recommendations may have to be intensified in patients with diabetes and/or CVD ${ }^{125}$ and physicians, in particular, may play an important role here.

The implementation of a healthy diet for each person, and thus at the population level, is, however, not the sole responsibility of physicians. The results of this thesis were shown in multiple settings and beneficial dietary modification of endothelial dysfunction and low-grade inflammation thus appears to play a role early in the development of CVD and at later stages during CVD progression. In fact this thesis supports the view that new population-wide strategies that improve diet should be introduced such as media and educational campaigns; labeling and consumer information; taxation, subsidies, and other economic incentives; school and workplace approaches; local environmental changes; and direct restrictions and mandates on unhealthy and healthy foods, respectively. ${ }^{131}$ Implementation of a healthy diet in the general population that favourably targets endothelial dysfunction and low-grade inflammation is important because even modest population shifts in CVD risk by a healthy diet substantially alter health outcomes. ${ }^{131}$

\section{Directions for future research (10.5)}

We have shown that a multiple-biomarker approach is an appropriate tool to investigate the associations between diet and endothelial dysfunction and low-grade inflammation in large cohort studies. This multi-marker approach could also be used to investigate associations between diet and other concepts than endothelial dysfunction and low-grade inflammation, such as biomarkers of vascular remodelling (matrix metalloproteinases) or biomarkers of adipose tissue metabolism (adipokines). ${ }^{132-145}$ The cohorts presented in this thesis are particularly valuable to investigate other related concepts because of the extensive characterization of 
vascular function, which enables investigation of interrelations between diet and new multi-marker concepts and the presently available CVD risk factors, such as endothelial dysfunction, low-grade inflammation and arterial stiffness. In addition, future studies could address other food groups or nutrient compounds that have been shown to be related to endothelial dysfunction and low-grade inflammation ${ }^{38-48,146}$ or that have been shown to play a role in CVD, for example sodium and potassium intake ${ }^{147}$, and investigate whether these food groups or nutrient compounds play a role in the development of CVD at the population level. These investigations will provide further insight into the complex relationship between diet and the development of CVD and might provide new targets for population-wide dietary recommendations.

The AGAHLS, The Hoorn and CODAM Studies are ongoing. We performed longitudinal investigations and follow-up will enable further elucidation of the temporality of the associations presented in this thesis. Nevertheless, new observational studies on diet and vascular function should be undertaken, because technological innovation enables more dietary components and more aspects of vascular function to be studied in detail. These studies could further unravel the complex interplay between diet and the development of CVD through investigation of e.g. dietary determinants of microvascular, macrovascular and cardiac function. Ideally, these new studies should repeatedly include extensive dietary exposure assessments and, concurrently, extensive vascular function assessments in order to fully elucidate the temporal aspects of these relationships. 


\section{References}

1. Aird WC. Phenotypic heterogeneity of the endothelium: I. Structure, function, and mechanisms. Circ Res. 2007;100:158-173.

2. Aird WC. Phenotypic heterogeneity of the endothelium: II. Representative vascular beds. Circ Res. 2007;100:174-190.

3. Ross R. Atherosclerosis--an inflammatory disease. N Engl J Med. 1999;340:115-126.

4. Borissoff JI, Spronk HM, ten Cate H. The hemostatic system as a modulator of atherosclerosis. N Engl J Med. 2011;364:1746-1760.

5. Lusis AJ. Atherosclerosis. Nature. 2000;407:233-241.

6. Hansson GK. Inflammation, atherosclerosis, and coronary artery disease. N Engl J Med. 2005;352: 1685-1695.

7. Yudkin JS, Stehouwer CD, Emeis JJ, Coppack SW. C-reactive protein in healthy subjects: associations with obesity, insulin resistance, and endothelial dysfunction: a potential role for cytokines originating from adipose tissue? Arterioscler Thromb Vasc Biol. 1999;19:972-978.

8. Schram MT, Chaturvedi N, Schalkwijk CG, Fuller JH, Stehouwer CD. Markers of inflammation are crosssectionally associated with microvascular complications and cardiovascular disease in type 1 diabetes-the EURODIAB Prospective Complications Study. Diabetologia. 2005;48:370-378.

9. de Jager J, Dekker JM, Kooy A, Kostense PJ, Nijpels G, Heine RJ, Bouter LM, Stehouwer CD. Endothelial dysfunction and low-grade inflammation explain much of the excess cardiovascular mortality in individuals with type 2 diabetes: the Hoorn Study. Arterioscler Thromb Vasc Biol. 2006;26:1086-1093.

10. Blankenberg S, Rupprecht HJ, Bickel C, Peetz D, Hafner G, Tiret L, Meyer J. Circulating cell adhesion molecules and death in patients with coronary artery disease. Circulation. 2001;104:1336-1342.

11. Morange PE, Simon C, Alessi MC, Luc G, Arveiler D, Ferrieres J, Amouyel P, Evans A, Ducimetiere P, Juhan-Vague I. Endothelial cell markers and the risk of coronary heart disease: the Prospective Epidemiological Study of Myocardial Infarction (PRIME) study. Circulation. 2004;109:1343-1348.

12. Ridker PM, Hennekens $\mathrm{CH}$, Buring JE, Rifai N. C-reactive protein and other markers of inflammation in the prediction of cardiovascular disease in women. N Engl J Med. 2000;342:836-843.

13. Becker A, van Hinsbergh VW, Jager A, Kostense PJ, Dekker JM, Nijpels G, Heine RJ, Bouter LM, Stehouwer $\mathrm{CD}$. Why is soluble intercellular adhesion molecule-1 related to cardiovascular mortality? Eur J Clin Invest. 2002;32:1-8.

14. Cao JJ, Arnold AM, Manolio TA, Polak JF, Psaty BM, Hirsch CH, Kuller LH, Cushman M. Association of carotid artery intima-media thickness, plaques, and C-reactive protein with future cardiovascular disease and all-cause mortality: the Cardiovascular Health Study. Circulation. 2007;116:32-38.

15. Jenny NS, Arnold AM, Kuller LH, Sharrett AR, Fried LP, Psaty BM, Tracy RP. Soluble intracellular adhesion molecule-1 is associated with cardiovascular disease risk and mortality in older adults. J Thromb Haemost. 2006;4:107-113.

16. Eldrup N, Kragelund C, Steffensen R, Nordestgaard BG. Prognosis by C-reactive protein and matrix metalloproteinase- 9 levels in stable coronary heart disease during 15 years of follow-up. Nutr Metab Cardiovasc Dis. 2012;22:677-683.

17. Kaplan RC, McGinn AP, Baird AE, Hendrix SL, Kooperberg C, Lynch J, Rosenbaum DM, Johnson KC, Strickler HD, Wassertheil-Smoller S. Inflammation and hemostasis biomarkers for predicting stroke in postmenopausal women: the Women's Health Initiative Observational Study. J Stroke Cerebrovasc Dis. 2008;17:344-355.

18. Luc G, Arveiler D, Evans A, Amouyel P, Ferrieres J, Bard JM, Elkhalil L, Fruchart JC, Ducimetiere P. Circulating soluble adhesion molecules ICAM-1 and VCAM-1 and incident coronary heart disease: the PRIME Study. Atherosclerosis. 2003;170:169-176.

19. Tzoulaki I, Murray GD, Lee AJ, Rumley A, Lowe GD, Fowkes FG. Relative value of inflammatory, hemostatic, and rheological factors for incident myocardial infarction and stroke: the Edinburgh Artery Study. Circulation. 2007;115:2119-2127.

20. Ridker PM, Rifai N, Stampfer MJ, Hennekens CH. Plasma concentration of interleukin- 6 and the risk of future myocardial infarction among apparently healthy men. Circulation. 2000;101:1767-1772. 
21. Ridker PM, Rifai N, Pfeffer M, Sacks F, Lepage S, Braunwald E. Elevation of tumor necrosis factor-alpha and increased risk of recurrent coronary events after myocardial infarction. Circulation. 2000;101: 2149-2153.

22. Ridker PM, Hennekens CH, Roitman-Johnson B, Stampfer MJ, Allen J. Plasma concentration of soluble intercellular adhesion molecule 1 and risks of future myocardial infarction in apparently healthy men. Lancet. 1998;351:88-92.

23. Jager A, van Hinsbergh VW, Kostense PJ, Emeis JJ, Yudkin JS, Nijpels G, Dekker JM, Heine RJ, Bouter LM, Stehouwer CD. von Willebrand factor, C-reactive protein, and 5-year mortality in diabetic and nondiabetic subjects: the Hoorn Study. Arterioscler Thromb Vasc Biol. 1999;19:3071-3078.

24. Frankel DS, Meigs JB, Massaro JM, Wilson PW, O'Donnell CJ, D'Agostino RB, Tofler GH. Von Willebrand factor, type 2 diabetes mellitus, and risk of cardiovascular disease: the framingham offspring study. Circulation. 2008;118:2533-2539.

25. Inaba Y, Chen JA, Bergmann SR. Prediction of future cardiovascular outcomes by flow-mediated vasodilatation of brachial artery: a meta-analysis. Int J Cardiovasc Imaging. 2010;26:631-640.

26. Mah E, Noh SK, Ballard KD, Matos ME, Volek JS, Bruno RS. Postprandial hyperglycemia impairs vascular endothelial function in healthy men by inducing lipid peroxidation and increasing asymmetric dimethylarginine:arginine. J Nutr. 2011;141:1961-1968.

27. Watanabe K, Oba K, Suzuki T, Ouchi M, Suzuki K, Futami-Suda S, Sekimizu K, Yamamoto N, Nakano H. Oral glucose loading attenuates endothelial function in normal individual. Eur J Clin Invest. 2011; 41:465-473.

28. Akbari CM, Saouaf R, Barnhill DF, Newman PA, LoGerfo FW, Veves A. Endothelium-dependent vasodilatation is impaired in both microcirculation and macrocirculation during acute hyperglycemia. J Vasc Surg. 1998;28:687-694.

29. Title LM, Cummings PM, Giddens K, Nassar BA. Oral glucose loading acutely attenuates endotheliumdependent vasodilation in healthy adults without diabetes: an effect prevented by vitamins $\mathrm{C}$ and $\mathrm{E}$. J Am Coll Cardiol. 2000;36:2185-2191.

30. Xiang GD, Sun HL, Zhao LS, Hou J, Yue L, Xu L. The antioxidant alpha-lipoic acid improves endothelial dysfunction induced by acute hyperglycaemia during OGTT in impaired glucose tolerance. Clin Endocrinol (Oxf). 2008;68:716-723.

31. Kawano H, Motoyama T, Hirashima O, Hirai N, Miyao Y, Sakamoto T, Kugiyama K, Ogawa H, Yasue H. Hyperglycemia rapidly suppresses flow-mediated endothelium-dependent vasodilation of brachial artery. J Am Coll Cardiol. 1999;34:146-154.

32. Harriss LR, English DR, Powles J, Giles GG, Tonkin AM, Hodge AM, Brazionis L, O'Dea K. Dietary patterns and cardiovascular mortality in the Melbourne Collaborative Cohort Study. Am J Clin Nutr. 2007;86:221-229.

33. Hu FB, Rimm EB, Stampfer MJ, Ascherio A, Spiegelman D, Willett WC. Prospective study of major dietary patterns and risk of coronary heart disease in men. Am J Clin Nutr. 2000;72:912-921.

34. Knoops KT, de Groot LC, Kromhout D, Perrin AE, Moreiras-Varela O, Menotti A, van Staveren WA. Mediterranean diet, lifestyle factors, and 10-year mortality in elderly European men and women: the HALE project. JAMA. 2004;292:1433-1439.

35. Mitrou PN, Kipnis V, Thiebaut AC, Reedy J, Subar AF, Wirfalt E, Flood A, Mouw T, Hollenbeck AR, Leitzmann MF, Schatzkin A. Mediterranean dietary pattern and prediction of all-cause mortality in a US population: results from the NIH-AARP Diet and Health Study. Arch Intern Med. 2007;167: 2461-2468.

36. Panagiotakos D, Pitsavos C, Chrysohoou C, Palliou K, Lentzas I, Skoumas I, Stefanadis C. Dietary patterns and 5-year incidence of cardiovascular disease: a multivariate analysis of the ATTICA study. Nutr Metab Cardiovasc Dis. 2009;19:253-263.

37. Hodge AM, English DR, Itsiopoulos C, O'Dea K, Giles GG. Does a Mediterranean diet reduce the mortality risk associated with diabetes: evidence from the Melbourne Collaborative Cohort Study. Nutr Metab Cardiovasc Dis. 2011;21:733-739.

38. De Caterina R. n-3 fatty acids in cardiovascular disease. N Engl J Med. 2011;364:2439-2450.

39. De Caterina R, Zampolli A, Del Turco S, Madonna R, Massaro M. Nutritional mechanisms that influence cardiovascular disease. Am J Clin Nutr. 2006;83:421S-426S.

40. Giugliano D, Ceriello A, Esposito K. The effects of diet on inflammation: emphasis on the metabolic syndrome. J Am Coll Cardiol. 2006;48:677-685. 
41. Davis N, Katz S, Wylie-Rosett J. The effect of diet on endothelial function. Cardiol Rev. 2007; 15:62-66.

42. Galland L. Diet and inflammation. Nutr Clin Pract. 2010;25:634-640.

43. Brown AA, Hu FB. Dietary modulation of endothelial function: implications for cardiovascular disease. Am J Clin Nutr. 2001;73:673-686.

44. Hall WL. Dietary saturated and unsaturated fats as determinants of blood pressure and vascular function. Nutr Res Rev. 2009;22:18-38.

45. Bau PF, Bau CH, Rosito GA, Manfroi WC, Fuchs FD. Alcohol consumption, cardiovascular health, and endothelial function markers. Alcohol. 2007;41:479-488.

46. Gresele P, Cerletti C, Guglielmini G, Pignatelli P, de Gaetano G, Violi F. Effects of resveratrol and other wine polyphenols on vascular function: an update. J Nutr Biochem. 2011;22:201-211.

47. Schini-Kerth VB, Auger C, Kim JH, Etienne-Selloum N, Chataigneau T. Nutritional improvement of the endothelial control of vascular tone by polyphenols: role of NO and EDHF. Pflugers Arch. 2010; 459:853-862.

48. Corti R, Flammer AJ, Hollenberg NK, Luscher TF. Cocoa and cardiovascular health. Circulation. 2009; 119:1433-1441.

49. Stehouwer CD, Gall MA, Twisk JW, Knudsen E, Emeis JJ, Parving HH. Increased urinary albumin excretion, endothelial dysfunction, and chronic low-grade inflammation in type 2 diabetes: progressive, interrelated, and independently associated with risk of death. Diabetes. 2002;51: 1157-1165.

50. Rothman KJ, Greenland S, Lash TL. Modern Epidemiology. Vol 3rd ed. Philadelphia: Lippincott Williams \& Wilkins 2008.

51. Cortes B, Nunez I, Cofan M, Gilabert R, Perez-Heras A, Casals E, Deulofeu R, Ros E. Acute effects of high-fat meals enriched with walnuts or olive oil on postprandial endothelial function. J Am Coll Cardiol. 2006;48:1666-1671.

52. Bai Y, Sun L, Yang T, Sun K, Chen J, Hui R. Increase in fasting vascular endothelial function after shortterm oral L-arginine is effective when baseline flow-mediated dilation is low: a meta-analysis of randomized controlled trials. Am J Clin Nutr. 2009;89:77-84.

53. MacMahon S, Peto R, Cutler J, Collins R, Sorlie P, Neaton J, Abbott R, Godwin J, Dyer A, Stamler J. Blood pressure, stroke, and coronary heart disease. Part 1, Prolonged differences in blood pressure: prospective observational studies corrected for the regression dilution bias. Lancet. 1990;335: 765-774.

54. Willett WC. Nutritional Epidemiology. 2th ed. New York, NY: Oxford University Press; 1998.

55. Thompson FE, Subar AF. Dietary assessment methodology. In: Coulston AM, Boushey CJ, eds. Nutrition in the prevention and treatment of disease. 2nd ed. San Diego, CA: Academic Press; 2008.

56. Bakker I, Twisk JW, van Mechelen W, Mensink GB, Kemper HC. Computerization of a dietary history interview in a running cohort; evaluation within the Amsterdam Growth and Health Longitudinal Study. Eur J Clin Nutr. 2003;57:394-404.

57. Kromhout D, de Lezenne Coulander C, Obermann-de Boer GL, van Kampen-Donker M, Goddijn E, Bloemberg BP. Changes in food and nutrient intake in middle-aged men from 1960 to 1985 (the Zutphen Study). Am J Clin Nutr. 1990;51:123-129.

58. Burke BS. The dietary history as a tool in research. J Am Diet Assoc. 1947;1041-1046.

59. Mensink GB, Haftenberger M, Thamm M. Validity of DISHES 98, a computerised dietary history interview: energy and macronutrient intake. Eur J Clin Nutr. 2001;55:409-417.

60. Thompson FE, Subar AF, Loria CM, Reedy JL, Baranowski T. Need for technological innovation in dietary assessment. J Am Diet Assoc. 2010;110:48-51.

61. Illner AK, Nothlings $\mathrm{U}$, Wagner $\mathrm{K}, \mathrm{Ward} \mathrm{H}$, Boeing $\mathrm{H}$. The assessment of individual usual food intake in large-scale prospective studies. Ann Nutr Metab. 2010;56:99-105.

62. Cade J, Thompson R, Burley V, Warm D. Development, validation and utilisation of food-frequency questionnaires - a review. Public Health Nutr. 2002;5:567-587.

63. Ocke MC, Bueno-de-Mesquita HB, Goddijn HE, Jansen A, Pols MA, van Staveren WA, Kromhout D. The Dutch EPIC food frequency questionnaire. I. Description of the questionnaire, and relative validity and reproducibility for food groups. Int J Epidemiol. 1997;26 Suppl 1:S37-48.

64. Ocke MC, Bueno-de-Mesquita HB, Pols MA, Smit HA, van Staveren WA, Kromhout D. The Dutch EPIC food frequency questionnaire. II. Relative validity and reproducibility for nutrients. Int J Epidemiol. 1997;26 Suppl 1:S49-58. 
65. Subar AF, Crafts J, Zimmerman TP, Wilson M, Mittl B, Islam NG, McNutt S, Potischman N, Buday R, Hull SG, Baranowski T, Guenther PM, Willis G, Tapia R, Thompson FE. Assessment of the accuracy of portion size reports using computer-based food photographs aids in the development of an automated self-administered 24-hour recall. J Am Diet Assoc. 2010;110:55-64.

66. Toeller M, Klischan A, Heitkamp G, Schumacher W, Milne R, Buyken A, Karamanos B, Gries FA. Nutritional intake of 2868 IDDM patients from 30 centres in Europe. EURODIAB IDDM Complications Study Group. Diabetologia. 1996;39:929-939.

67. Toeller M, Buyken A, Heitkamp G, Milne R, Klischan A, Gries FA. Repeatability of three-day dietary records in the EURODIAB IDDM Complications Study. Eur J Clin Nutr. 1997;51:74-80.

68. Toeller M, Buyken A, Heitkamp G, Bramswig S, Mann J, Milne R, Gries FA, Keen H. Protein intake and urinary albumin excretion rates in the EURODIAB IDDM Complications Study. Diabetologia. 1997; 40:1219-1226.

69. Buyken AE, Toeller M, Heitkamp G, Vitelli F, Stehle P, Scherbaum WA, Fuller JH. Relation of fibre intake to $\mathrm{HbA} 1 \mathrm{c}$ and the prevalence of severe ketoacidosis and severe hypoglycaemia. EURODIAB IDDM Complications Study Group. Diabetologia. 1998;41:882-890.

70. Mozaffarian D, Hao T, Rimm EB, Willett WC, Hu FB. Changes in diet and lifestyle and long-term weight gain in women and men. N Engl J Med. 2011;364:2392-2404.

71. Henry RM, Ferreira I, Kostense PJ, Dekker JM, Nijpels G, Heine RJ, Kamp O, Bouter LM, Stehouwer CD. Type 2 diabetes is associated with impaired endothelium-dependent, flow-mediated dilation, but impaired glucose metabolism is not; The Hoorn Study. Atherosclerosis. 2004;174:49-56.

72. de Koning L, Liptak C, Shkreta A, Bradwin G, Hu FB, Pradhan AD, Rifai N, Kellogg MD. A multiplex immunoassay gives different results than singleplex immunoassays which may bias epidemiologic associations. Clin Biochem. 2012;45:848-851.

73. Hartweg J, Gunter M, Perera R, Farmer A, Cull C, Schalkwijk C, Kok A, Twaalfhoven H, Holman R, Neil A. Stability of soluble adhesion molecules, selectins, and C-reactive protein at various temperatures: implications for epidemiological and large-scale clinical studies. Clin Chem. 2007;53:1858-1860.

74. Hutchinson WL, Koenig W, Frohlich M, Sund M, Lowe GD, Pepys MB. Immunoradiometric assay of circulating C-reactive protein: age-related values in the adult general population. Clin Chem. 2000; 46:934-938.

75. Black MA, Cable NT, Thijssen DH, Green DJ. Importance of measuring the time course of flowmediated dilatation in humans. Hypertension. 2008;51:203-210.

76. Thijssen DH, Black MA, Pyke KE, Padilla J, Atkinson G, Harris RA, Parker BA, Widlansky ME, Tschakovsky ME, Green DJ. Assessment of flow mediated dilation (FMD) in humans: a methodological and technical guideline. Am J Physiol Heart Circ Physiol. 2011;300:H2-12.

77. Donald AE, Halcox JP, Charakida M, Storry C, Wallace SM, Cole TJ, Friberg P, Deanfield JE. Methodological approaches to optimize reproducibility and power in clinical studies of flow-mediated dilation. J Am Coll Cardiol. 2008;51:1959-1964.

78. Sorensen KE, Celermajer DS, Spiegelhalter DJ, Georgakopoulos D, Robinson J, Thomas O, Deanfield JE. Non-invasive measurement of human endothelium dependent arterial responses: accuracy and reproducibility. Br Heart J. 1995;74:247-253.

79. Welsch MA, Allen JD, Geaghan JP. Stability and reproducibility of brachial artery flow-mediated dilation. Med Sci Sports Exerc. 2002;34:960-965.

80. Woodman RJ, Playford DA, Watts GF, Cheetham C, Reed C, Taylor RR, Puddey IB, Beilin LJ, Burke V, Mori TA, Green D. Improved analysis of brachial artery ultrasound using a novel edge-detection software system. J Appl Physiol. 2001;91:929-937.

81. Ghiadoni L, Faita F, Salvetti M, Cordiano C, Biggi A, Puato M, Di Monaco A, De Siati L, Volpe M, Ambrosio G, Gemignani V, Muiesan ML, Taddei S, Lanza GA, Cosentino F. Assessment of flowmediated dilation reproducibility: a nationwide multicenter study. J Hypertens. 2012;30:1399-1405.

82. Corretti MC, Anderson TJ, Benjamin EJ, Celermajer D, Charbonneau F, Creager MA, Deanfield J, Drexler H, Gerhard-Herman M, Herrington D, Vallance P, Vita J, Vogel R. Guidelines for the ultrasound assessment of endothelial-dependent flow-mediated vasodilation of the brachial artery: a report of the International Brachial Artery Reactivity Task Force. J Am Coll Cardiol. 2002;39:257-265.

83. Celermajer DS, Sorensen KE, Gooch VM, Spiegelhalter DJ, Miller OI, Sullivan ID, Lloyd JK, Deanfield JE. Non-invasive detection of endothelial dysfunction in children and adults at risk of atherosclerosis. Lancet. 1992;340:1111-1115. 
84. Parker BA, Trehearn TL, Meendering JR. Pick your Poiseuille: normalizing the shear stimulus in studies of flow-mediated dilation. J Appl Physiol. 2009;107:1357-1359.

85. Laurent S, Cockcroft J, Van Bortel L, Boutouyrie P, Giannattasio C, Hayoz D, Pannier B, Vlachopoulos C, Wilkinson I, Struijker-Boudier H. Expert consensus document on arterial stiffness: methodological issues and clinical applications. Eur Heart J. 2006;27:2588-2605.

86. Schouten F, Twisk JW, de Boer MR, Stehouwer CD, Serne EH, Smulders YM, Ferreira I. Increases in central fat mass and decreases in peripheral fat mass are associated with accelerated arterial stiffening in healthy adults: the Amsterdam Growth and Health Longitudinal Study. Am J Clin Nutr. 2011;94:40-48.

87. Henry RM, Kostense PJ, Spijkerman AM, Dekker JM, Nijpels G, Heine RJ, Kamp O, Westerhof N, Bouter LM, Stehouwer CD. Arterial stiffness increases with deteriorating glucose tolerance status: the Hoorn Study. Circulation. 2003;107:2089-2095.

88. Ferreira I, Henry RM, Twisk JW, van Mechelen W, Kemper HC, Stehouwer CD. The metabolic syndrome, cardiopulmonary fitness, and subcutaneous trunk fat as independent determinants of arterial stiffness: the Amsterdam Growth and Health Longitudinal Study. Arch Intern Med. 2005; 165:875-882.

89. Franklin SS, Khan SA, Wong ND, Larson MG, Levy D. Is pulse pressure useful in predicting risk for coronary heart Disease? The Framingham heart study. Circulation. 1999;100:354-360.

90. Calder PC, Ahluwalia N, Brouns F, Buetler T, Clement K, Cunningham K, Esposito K, Jonsson LS, Kolb H, Lansink M, Marcos A, Margioris A, Matusheski N, Nordmann H, O'Brien J, Pugliese G, Rizkalla S, Schalkwijk C, Tuomilehto J, Warnberg J, Watzl B, Winklhofer-Roob BM. Dietary factors and low-grade inflammation in relation to overweight and obesity. Br J Nutr. 2011;106 Suppl 3:S5-78.

91. Esmaillzadeh A, Azadbakht L. Home use of vegetable oils, markers of systemic inflammation, and endothelial dysfunction among women. Am J Clin Nutr. 2008;88:913-921.

92. Song Y, Li TY, van Dam RM, Manson JE, Hu FB. Magnesium intake and plasma concentrations of markers of systemic inflammation and endothelial dysfunction in women. Am J Clin Nutr. 2007; 85:1068-1074.

93. Wannamethee SG, Lowe GD, Rumley A, Bruckdorfer KR, Whincup PH. Associations of vitamin C status, fruit and vegetable intakes, and markers of inflammation and hemostasis. Am J Clin Nutr. 2006; 83:567-574; quiz 726-567.

94. Abramson JL, Weintraub WS, Vaccarino V. Association between pulse pressure and C-reactive protein among apparently healthy US adults. Hypertension. 2002;39:197-202.

95. Amar J, Ruidavets JB, Bal Dit Sollier C, Bongard V, Boccalon H, Chamontin B, Drouet L, Ferrieres J. Soluble CD14 and aortic stiffness in a population-based study. J Hypertens. 2003;21:1869-1877.

96. Amar J, Ruidavets JB, Sollier CB, Bongard V, Boccalon H, Chamontin B, Drouet L, Ferrieres J. Relationship between $C$ reactive protein and pulse pressure is not mediated by atherosclerosis or aortic stiffness. J Hypertens. 2004;22:349-355.

97. Chae CU, Lee RT, Rifai N, Ridker PM. Blood pressure and inflammation in apparently healthy men. Hypertension. 2001;38:399-403.

98. Eklund C, Kivimaki M, Islam MS, Juonala M, Kahonen M, Marniemi J, Lehtimaki T, Viikari J, Raitakari OT, Hurme M. C-reactive protein genetics is associated with carotid artery compliance in men in The Cardiovascular Risk in Young Finns Study. Atherosclerosis. 2008;196:841-848.

99. Juonala M, Jarvisalo MJ, Maki-Torkko N, Kahonen M, Viikari JS, Raitakari OT. Risk factors identified in childhood and decreased carotid artery elasticity in adulthood: the Cardiovascular Risk in Young Finns Study. Circulation. 2005;112:1486-1493.

100. Kampus P, Kals J, Ristimae T, Fischer K, Zilmer M, Teesalu R. High-sensitivity C-reactive protein affects central haemodynamics and augmentation index in apparently healthy persons. J Hypertens. 2004; 22:1133-1139.

101. Kampus P, Kals J, Ristimae T, Muda P, Ulst K, Zilmer K, Salonen RM, Tuomainen TP, Teesalu R, Zilmer $M$. Augmentation index and carotid intima-media thickness are differently related to age, C-reactive protein and oxidized low-density lipoprotein. J Hypertens. 2007;25:819-825.

102. Kullo IJ, Seward JB, Bailey KR, Bielak LF, Grossardt BR, Sheedy PF, 2nd, Peyser PA, Turner ST. Creactive protein is related to arterial wave reflection and stiffness in asymptomatic subjects from the community. Am J Hypertens. 2005;18:1123-1129. 
103. Lieb W, Larson MG, Benjamin EJ, Yin X, Tofler GH, Selhub J, Jacques PF, Wang TJ, Vita JA, Levy D, Vasan RS, Mitchell GF. Multimarker approach to evaluate correlates of vascular stiffness: the Framingham Heart Study. Circulation. 2009;119:37-43.

104. Mattace-Raso FU, van der Cammen TJ, van der Meer IM, Schalekamp MA, Asmar R, Hofman A, Witteman JC. C-reactive protein and arterial stiffness in older adults: the Rotterdam Study. Atherosclerosis. 2004;176:111-116.

105. Nakhai-Pour HR, Grobbee DE, Bots ML, Muller M, van der Schouw YT. C-reactive protein and aortic stiffness and wave reflection in middle-aged and elderly men from the community. J Hum Hypertens. 2007;21:949-955.

106. Schnabel R, Larson MG, Dupuis J, Lunetta KL, Lipinska I, Meigs JB, Yin X, Rong J, Vita JA, Newton-Cheh C, Levy D, Keaney JF, Jr., Vasan RS, Mitchell GF, Benjamin EJ. Relations of inflammatory biomarkers and common genetic variants with arterial stiffness and wave reflection. Hypertension. 2008; 51:1651-1657.

107. Schumacher W, Cockcroft J, Timpson NJ, McEniery CM, Gallacher J, Rumley A, Lowe G, Smith GD, Wilkinson IB, Ben-Shlomo Y. Association between C-reactive protein genotype, circulating levels, and aortic pulse wave velocity. Hypertension. 2009;53:150-157.

108. Schutte R, Schutte AE, Van Rooyen JM, Huisman HW, Palmer IM, Fourie CM, Peter S, Malan L, Malan NT, Reimann M. Von Willebrand factor as marker of vascular function in South African women: the POWIRS Study. Am J Hypertens. 2008;21:1298-1303.

109. Whincup PH, Gilg JA, Donald AE, Katterhorn M, Oliver C, Cook DG, Deanfield JE. Arterial distensibility in adolescents: the influence of adiposity, the metabolic syndrome, and classic risk factors. Circulation. 2005;112:1789-1797.

110. Yasmin, McEniery CM, Wallace S, Mackenzie IS, Cockcroft JR, Wilkinson IB. C-reactive protein is associated with arterial stiffness in apparently healthy individuals. Arterioscler Thromb Vasc Biol. 2004;24:969-974.

111. Hill B. The environment and Disease: Association or Causation. 1965.

112. Twisk JWR. Applied Longitudinal Data Analysis for Epidemiology: A Practical Guide. 1st ed. Cambridge, United Kingdom: Cambridge University; 2003.

113. Nagel G, Zoller D, Ruf T, Rohrmann S, Linseisen J. Long-term reproducibility of a food-frequency questionnaire and dietary changes in the European Prospective Investigation into Cancer and Nutrition (EPIC)-Heidelberg cohort. Br J Nutr. 2007;98:194-200.

114. Nettleton JA, Steffen LM, Mayer-Davis EJ, Jenny NS, Jiang R, Herrington DM, Jacobs DR, Jr. Dietary patterns are associated with biochemical markers of inflammation and endothelial activation in the Multi-Ethnic Study of Atherosclerosis (MESA). Am J Clin Nutr. 2006;83:1369-1379.

115. Lopez-Garcia E, Schulze MB, Fung TT, Meigs JB, Rifai N, Manson JE, Hu FB. Major dietary patterns are related to plasma concentrations of markers of inflammation and endothelial dysfunction. Am J Clin Nutr. 2004;80:1029-1035.

116. Fung TT, McCullough ML, Newby PK, Manson JE, Meigs JB, Rifai N, Willett WC, Hu FB. Diet-quality scores and plasma concentrations of markers of inflammation and endothelial dysfunction. Am J Clin Nutr. 2005;82:163-173.

117. He K, Liu K, Daviglus ML, Jenny NS, Mayer-Davis E, Jiang R, Steffen L, Siscovick D, Tsai M, Herrington D. Associations of dietary long-chain n-3 polyunsaturated fatty acids and fish with biomarkers of inflammation and endothelial activation (from the Multi-Ethnic Study of Atherosclerosis [MESA]). Am J Cardiol. 2009;103:1238-1243.

118. Gao X, Bermudez Ol, Tucker KL. Plasma C-reactive protein and homocysteine concentrations are related to frequent fruit and vegetable intake in Hispanic and non-Hispanic white elders. J Nutr. 2004; 134:913-918.

119. Lopez-Garcia E, Schulze MB, Meigs JB, Manson JE, Rifai N, Stampfer MJ, Willett WC, Hu FB. Consumption of trans fatty acids is related to plasma biomarkers of inflammation and endothelial dysfunction. J Nutr. 2005;135:562-566.

120. Shai I, Rimm EB, Schulze MB, Rifai N, Stampfer MJ, Hu FB. Moderate alcohol intake and markers of inflammation and endothelial dysfunction among diabetic men. Diabetologia. 2004;47:1760-1767.

121. Greenwald SE. Ageing of the conduit arteries. J Pathol. 2007;211:157-172.

122. Stehouwer CD, Henry RM, Ferreira I. Arterial stiffness in diabetes and the metabolic syndrome: a pathway to cardiovascular disease. Diabetologia. 2008;51:527-539. 
123. McEniery CM, Qasem A, Schmitt M, Avolio AP, Cockcroft JR, Wilkinson IB. Endothelin-1 regulates arterial pulse wave velocity in vivo. J Am Coll Cardiol. 2003;42:1975-1981.

124. Wilkinson IB, Qasem A, McEniery CM, Webb DJ, Avolio AP, Cockcroft JR. Nitric oxide regulates local arterial distensibility in vivo. Circulation. 2002;105:213-217.

125. Lichtenstein $A H$, Appel $\amalg$, Brands $M$, Carnethon M, Daniels S, Franch HA, Franklin B, Kris-Etherton $P$, Harris WS, Howard B, Karanja N, Lefevre M, Rudel L, Sacks F, Van Horn L, Winston M, Wylie-Rosett J. Diet and lifestyle recommendations revision 2006: a scientific statement from the American Heart Association Nutrition Committee. Circulation. 2006;114:82-96.

126. Yousuf O, Mohanty BD, Martin SS, Joshi PH, Blaha MJ, Nasir K, Blumenthal RS, Budoff MJ. Highsensitivity C-reactive protein and cardiovascular disease: a resolute belief or an elusive link? J Am Coll Cardiol. 2013;62:397-408.

127. Ter Avest E, Stalenhoef AF, de Graaf J. What is the role of non-invasive measurements of atherosclerosis in individual cardiovascular risk prediction? Clin Sci (Lond). 2007;112:507-516.

128. Den Ruijter HM, Peters SA, Anderson TJ, Britton AR, Dekker JM, Eijkemans MJ, Engstrom G, Evans GW, de Graaf J, Grobbee DE, Hedblad B, Hofman A, Holewijn S, Ikeda A, Kavousi M, Kitagawa K, Kitamura A, Koffijberg H, Lonn EM, Lorenz MW, Mathiesen EB, Nijpels G, Okazaki S, O'Leary DH, Polak JF, Price JF, Robertson C, Rembold CM, Rosvall M, Rundek T, Salonen JT, Sitzer M, Stehouwer CD, Witteman JC, Moons KG, Bots ML. Common carotid intima-media thickness measurements in cardiovascular risk prediction: a meta-analysis. Jama. 2012;308:796-803.

129. Peters SA, Grobbee DE, Bots ML. Carotid intima-media thickness: a suitable alternative for cardiovascular risk as outcome? Eur J Cardiovasc Prev Rehabil. 2011;18:167-174.

130. Stehouwer CD. Is measurement of endothelial dysfunction clinically useful? Eur J Clin Invest. 1999; 29:459-461.

131. Mozaffarian D, Afshin A, Benowitz NL, Bittner V, Daniels SR, Franch HA, Jacobs DR, Jr., Kraus WE, KrisEtherton PM, Krummel DA, Popkin BM, Whitsel LP, Zakai NA. Population Approaches to Improve Diet, Physical Activity, and Smoking Habits: A Scientific Statement From the American Heart Association. Circulation. 2012;126:1514-1563.

132. Safar ME, O'Rourke MF. Handbook of Hypertension: Arterial Stiffness in Hypertension. Vol 23. Amsterdam, Netherlands: Elsevier B.V.; 2006.

133. Boger $\mathrm{RH}$. The emerging role of asymmetric dimethylarginine as a novel cardiovascular risk factor. Cardiovasc Res. 2003;59:824-833.

134. de Valk-de Roo GW, Stehouwer CD, Lambert J, Schalkwijk CG, van der Mooren MJ, Kluft C, Netelenbos C. Plasma homocysteine is weakly correlated with plasma endothelin and von Willebrand factor but not with endothelium-dependent vasodilatation in healthy postmenopausal women. Clin Chem. 1999;45:1200-1205.

135. Devaraj S, Xu DY, Jialal I. C-reactive protein increases plasminogen activator inhibitor-1 expression and activity in human aortic endothelial cells: implications for the metabolic syndrome and atherothrombosis. Circulation. 2003;107:398-404.

136. Eaton CB, Gramling R, Parker DR, Roberts MB, Lu B, Ridker PM. Prospective association of vascular endothelial growth factor-A (VEGF-A) with coronary heart disease mortality in Southeastern New England. Atherosclerosis. 2008.

137. Hill JM, Zalos G, Halcox JP, Schenke WH, Waclawiw MA, Quyyumi AA, Finkel T. Circulating endothelial progenitor cells, vascular function, and cardiovascular risk. N Engl J Med. 2003;348:593-600.

138. Lekakis J, Abraham P, Balbarini A, Blann A, Boulanger CM, Cockcroft J, Cosentino F, Deanfield J, Gallino A, Ikonomidis I, Kremastinos D, Landmesser U, Protogerou A, Stefanadis C, Tousoulis D, Vassalli G, Vink H, Werner N, Wilkinson I, Vlachopoulos C. Methods for evaluating endothelial function: a position statement from the European Society of Cardiology Working Group on Peripheral Circulation. Eur J Cardiovasc Prev Rehabil. 2011;18:775-789.

139. Deanfield J, Donald A, Ferri C, Giannattasio C, Halcox J, Halligan S, Lerman A, Mancia G, Oliver JJ, Pessina AC, Rizzoni D, Rossi GP, Salvetti A, Schiffrin EL, Taddei S, Webb DJ. Endothelial function and dysfunction. Part I: Methodological issues for assessment in the different vascular beds: a statement by the Working Group on Endothelin and Endothelial Factors of the European Society of Hypertension. J Hypertens. 2005;23:7-17. 
140. Yasmin, McEniery CM, Wallace S, Dakham Z, Pulsalkar P, Maki-Petaja K, Ashby MJ, Cockcroft JR, Wilkinson IB. Matrix metalloproteinase-9 (MMP-9), MMP-2, and serum elastase activity are associated with systolic hypertension and arterial stiffness. Arterioscler Thromb Vasc Biol. 2005; 25:372.

141. Ingelsson E, Hulthe J, Lind L. Inflammatory markers in relation to insulin resistance and the metabolic syndrome. Eur J Clin Invest. 2008;38:502-509.

142. Tedgui A, Mallat Z. Cytokines in atherosclerosis: pathogenic and regulatory pathways. Physiol Rev. 2006;86:515-581.

143. Nin JW, Ferreira I, Schalkwijk CG, Prins MH, Chaturvedi N, Fuller JH, Stehouwer CD. Levels of soluble receptor for AGE are cross-sectionally associated with cardiovascular disease in type 1 diabetes, and this association is partially mediated by endothelial and renal dysfunction and by low-grade inflammation: the EURODIAB Prospective Complications Study. Diabetologia. 2009;52:705-714.

144. Schnabel RB, Larson MG, Yamamoto JF, Kathiresan S, Rong J, Levy D, Keaney JF, Jr., Wang TJ, Vasan RS, Benjamin EJ. Relation of multiple inflammatory biomarkers to incident atrial fibrillation. Am J Cardiol. 2009;104:92-96.

145. Kershaw EE, Flier JS. Adipose tissue as an endocrine organ. J Clin Endocrinol Metab. 2004;89: 2548-2556.

146. Qi L, van Dam RM, Liu S, Franz M, Mantzoros C, Hu FB. Whole-grain, bran, and cereal fiber intakes and markers of systemic inflammation in diabetic women. Diabetes Care. 2006;29:207-211.

147. Adrogue HJ, Madias NE. Sodium and potassium in the pathogenesis of hypertension. N Engl J Med. 2007;356:1966-1978. 
Summary (samenvatting voor de leek) 


\section{Samenvatting}

Dit proefschrift beschrijft het onderzoek naar de vraag of endotheeldisfunctie en laaggradige ontsteking bepaald worden door dieet en een oorzaak zijn van slagaderstijfheid.

Het vasculaire endotheel bestaat uit een enkelvoudige laag cellen aan de binnenkant van alle bloedvaten in ons lichaam. In totaal zijn dat ongeveer 10-60 biljoen cellen. Het vasculaire endotheel reageert op veel verschillende stimuli en voert meerdere functies uit. Dit gebeurt op een heterogene manier door ons gehele bloedvatstelsel. Het vasculaire endotheel is de primaire plaats van disfunctie in veel ziektebeelden, zoals hart- en vaatziekten. Endotheeldisfunctie kan gedefinieerd worden als een verandering in normale endotheliale reacties en/of functies die samengaan met slechter functioneren van één orgaan of meerdere organen. Endotheeldisfunctie heeft daarom veel dimensies. Ontstekingscellen van het immuunsysteem gaan een interactie aan met het vasculaire endotheel. Endotheeldisfunctie en laaggradige ontsteking zijn daarom nauw gerelateerd. Ze beïnvloeden zowel het ontstaan als het beloop van hart- en vaatziekten. Daarom zijn endotheeldisfunctie en laaggradige ontsteking belangrijke aangrijpingsmechanismen om hart- en vaatziekten te voorkómen en/of te verminderen.

Dieet is wellicht één van de belangrijkste beïnvloedbare risicofactoren voor het ontstaan en het voortschrijden van hart- en vaatziekten, omdat een gezond dieet samenhangt met minder hart- en vaatziekten. Endotheeldisfunctie en laaggradige ontsteking zouden wel eens de mechanismen kunnen zijn waarlangs een gezond dieet hart- en vaatziekten voorkómt en/of vermindert. Dit proefschrift beschrijft het onderzoek naar het verband tussen dieet en endotheeldisfunctie en laaggradige ontsteking in verschillende populaties met en zonder hart- en vaatziekten.

Endotheeldisfunctie en laaggradige ontsteking voorspellen het ontstaan van hart- en vaatziekten. Gedeeltelijk kan dit verband verklaard worden door het feit dat endotheeldisfunctie en laaggradige ontsteking een sleutelrol spelen bij slagadervervetting en trombose. Andere mechanismen die samenhangen met het ontstaan van hart- en vaatziekten, zoals slagaderverstijving, zouden ook een rol kunnen spelen in dit verband. Daarom worden de verbanden tussen, aan de ene kant, endotheeldisfunctie en laaggradige ontsteking en, aan de andere kant, slagaderstijfheid in dit proefschrift onderzocht.

Het doel van dit proefschrift was bewijs te vinden voor de centrale doelstelling dat endotheeldisfunctie en laaggradige ontsteking bepaald worden door dieet en een oorzaak zijn van slagaderstijfheid. We onderzochten verschillende grote populaties van mensen mét en zonder hart- en vaatziekten en/of diabetes (ofwel suikerziekte). 
Uit (eerder) opgeslagen bloedmonsters van de deelnemers bepaalden we meerdere biomarkers van endotheeldisfunctie (ieder toe te schrijven aan één van de vele soorten van endotheeldisfunctie) en laaggradige ontsteking. Ook namen wij vragenlijsten af en voerden we interviews uit om voedselinname te meten. Daarnaast gebruikten we ultrageluidtechnieken om de mate van stijfheid van slagaders vast te stellen. Op deze manier was het mogelijk de centrale doelstelling te onderzoeken in vrijlevende mensen over een groot leeftijdsbereik, in gezondheid en ziekte, waarbij we zowel dwarsdoorsnede onderzoek als onderzoek over de tijd uitvoerden.

\section{Belangrijkste bevindingen}

Voordat we de centrale doelstelling van dit proefschrift onderzochten, hebben we in deel 1 (hoofdstukken 2 en 3 ) eerst onderzoek gedaan naar het meten van biomarkers van endotheeldisfunctie en laaggradige ontsteking.

In hoofdstuk 2 voerden we in 574 deelnemers van de CODAM Studie een onderzoek uit waarin we meetmethoden voor de bepaling van biomarkers in bloedmonsters vergeleken. We vergeleken 'nieuwe' multiplex technologie met bestaande, enkelvoudige methoden om vast te stellen of de nieuwe, meer efficiënte, multiplex technologie gebruikt zou kunnen worden in (lopende) populatiestudies. De resultaten lieten zien dat absolute concentraties van vier onderzochte biomarkers (CRP, SAA, sICAM-1 en SVCAM-1) verschillend waren tussen de bestaande, enkelvoudige methoden en de nieuwe multiplex technologie. Het verschil in absolute concentraties tussen de methoden werd vastgesteld en de vergelijkingen uit deze analyse kunnen gebruikt worden om de twee methoden in elkaar om te rekenen. De verbanden tussen, aan de ene kant, individuele biomarkers en, aan de andere kant, glucose metabolisme, lichaamsgewicht, het metabool syndroom en roken waren niet verschillend tussen beide meetmethoden. Wij concludeerden dat de nieuwe multiplex technologie aantrekkelijk is voor het efficiënt meten van meerdere biomarkers. Deze multiplex technologie werd toegepast in de populatiestudies in dit proefschrift.

In hoofdstuk 3 onderzochten we in 30 gezonde mannen bloedstroomgemedieerde vaatwandverwijding als functionele biomarker van endotheeldisfunctie. Een grotere verwijding van de slagader geeft een betere endotheelfunctie weer. Deze verwijding van de slagader wordt veroorzaakt door een grotere bloedstroom die in deze studie experimenteel opgewekt werd. Dit werkt als volgt: de toegenomen bloedstroom oefent een wrijvingskracht uit op de aanliggende endotheelcellen die daarop reageren met het uitscheiden van chemische stoffen die op hun beurt zorgen dat de spiercellen in de slagaderwand ontspannen. We rapporteerden dat het meten van bloedstroomgemedieerde vaatwandverwijding middels het meten van de diameterverandering van het bloedvat verbeterd kan worden door de bloedstroomsnelheid mee te nemen in de berekeningen. We onderzochten twee aspecten van het meten; de meettechniek zelf en het meten van een biologische proces. Op het meettechnische vlak lieten de resultaten zien dat het meten van de gebruikelijke 
maximale diameterverwijding en van 'nieuwe' indexen die ook de bloedstroomsnelheid meenamen vergelijkbaar was. Biologisch gezien is het van belang dat de verandering in bloedstroomgemedieerde vaatwandverwijding, na het toedienen van een standaard glucosedrank gemakkelijker meetbaar was wanneer de bloedstroomstimulus meegenomen werd. Onze studie kon niet aantonen dat dit effect veroorzaakt werd door de glucose in de drank. Dit onderzoek geeft aan dat het meten van functionele biomarkers van endotheeldisfunctie complex is en dat het meenemen van de bloedstroomsnelheid in de berekening een belangrijke verbetering lijkt te zijn voor het meten van bloedstroomgemedieerde vaatwandverwijding.

In deel 2 (hoofdstukken 4-7) onderzochten we de verbanden tussen vijf voedselgroepen (vis, fruit, groenten, alcoholische dranken en melkproducten) en (grotendeels) identieke, uitgebreide sets van biomarkers van endotheeldisfunctie en laaggradige ontsteking in verschillende populaties: in gezonde jonge volwassenen (hoofdstuk 4), in oudere mensen (hoofdstukken 5-6) en in individuen met diabetes mellitus (type 1 of type 2) en/of hart- en vaatziekten (hoofdstukken 5-7). Daarbij maakten we gebruik van herhaalde metingen van het dieet (hoofdstukken 6-7) en/of herhaalde metingen van biomarkers (hoofdstukken 4 en 6 ).

In hoofdstuk 4 lieten we zien dat in 301 gezonde jonge volwassenen van het Amsterdamse Groei en Gezondheidsonderzoek meer visconsumptie, maar geen van de andere voedselgroepen, samenhing met afgenomen endotheeldisfunctie en afgenomen laaggradige ontsteking over een periode van 6 jaar. De inname van omega-3 vetzuren, welke voornamelijk in vis voorkomen, hing samen met afgenomen endotheeldisfunctie en afgenomen laaggradige ontsteking. Deze omega-3 visvetzuren verklaarden $83 \%$ van het verband tussen hogere visconsumptie en de afname in endotheeldisfunctie en $40 \%$ van het verband tussen hogere visconsumptie en de afname in laaggradige ontsteking. Belangrijk is dat deze resultaten onafhankelijk waren van de inname van andere voeding en leefstijl.

In hoofdstuk 5 werd het bovenstaande onderzoek herhaald in een dwarsdoorsnede onderzoek in 801 oudere individuen van de Hoorn Studie. Naast de in bloedmonsters gemeten biomarkers werd nu ook bloedstroomgemedieerde vaatwandverwijding als maat voor endotheeldisfunctie meegenomen. In deze studie hielden de consumptie van alcohol en rode wijn verband met lagere endotheeldisfunctie. De verbanden voor bloedstroomgemedieerde vaatwandverwijding waren echter minder duidelijk aanwezig dan die voor biomarkers gemeten in bloedmonsters. Geen van de voedingsgroepen hield verband met laaggradige ontsteking, behalve de consumptie van rode wijn. Dit verband tussen meer consumptie van rode wijn en minder laaggradige ontsteking was sterker in deelnemers met diabetes mellitus type 2 en/of hart- en vaatziekten, vergeleken met deelnemers zonder deze ziekten. Deze resultaten waren onafhankelijk van de inname van andere voeding en leefstijl.

In hoofdstuk 6 onderzochten we de eerder genoemde verbanden tussen de vijf voedselgroepen en endotheeldisfunctie en laaggradige ontsteking over een periode 
van 7 jaar, in 557 deelnemers met een verhoogd risico op hart- en vaatziekten en diabetes mellitus van de CODAM Studie. We lieten zien dat een hogere consumptie van vis samenhing met minder endotheeldisfunctie, maar dat er geen verband was voor melkproducten, fruit, groenten en alcoholische dranken met endotheeldisfunctie. Aanvullende analyses met componenten van deze voedselgroepen lieten zien dat een hogere consumptie van magere vis, rauwe groenten, en een lagere consumptie van vette melkproducten $(>2 \%$ vet) verband hielden met minder endotheeldisfunctie. Geen van de vijf hoofdvoedselgroepen hing samen met laaggradige ontsteking. Echter, aanvullende analyses met componenten van deze voedselgroepen lieten zien dat een hogere consumptie van wijn en een lagere consumptie van sterke drank verband hielden met minder laaggradige ontsteking. Deze verbanden waren minder duidelijk aanwezig voor laaggradige ontsteking dan voor endotheeldisfunctie. Aanvullende analyses lieten zien dat de consumptie van vers fruit gunstig was en dat de consumptie van vette melkproducten ongunstig was met betrekking tot laaggradige ontsteking, maar dan alleen in individuen met diabetes mellitus type 2 en/of hart- en vaatziekten. Belangrijk is dat deze resultaten onafhankelijk waren van de inname van andere voeding en leefstijl.

In hoofdstuk 7 waren gegevens op het niveau van voedselgroepen niet beschikbaar en daarom werd als alternatief de inname van nutriënten onderzocht. Wij vonden dat diegene van 491 volwassen patiënten met diabetes mellitus type 1 van de EURODIAB Studie die meer endotheeldisfunctie hadden, minder (oplosbare en onoplosbare) vezels, minder meervoudig onverzadigde vetten en minder plantaardig eiwit consumeerden over een periode van 8 voorgaande jaren. Er waren geen verbanden met koolhydraten, (andere types van) vet, cholesterol of (dierlijk) eiwit. Verder bleek dat individuen met meer laaggradige ontsteking minder vezels, minder oplosbare vezels, minder meervoudig onverzadigde vetten en meer cholesterol consumeerden over de 8 voorgaande jaren. Er waren geen associaties met onoplosbare vezels, koolhydraten, (andere types van) vet, of (enigerlei soort van) eiwit. Deze resultaten waren onafhankelijk van de inname van andere nutriënten en leefstijl.

Uiteindelijk, in deel 3 (hoofdstukken 8 en 9) onderzochten we of (grotendeels) identieke, uitgebreide sets van biomarkers van endotheeldisfunctie en laaggradige ontsteking verband hielden met grotere stijfheid van slagaders in verschillende populaties: in gezonde jonge volwassenen (hoofdstuk 8) en in oudere mensen (hoofdstuk 9). Daarbij maakten we gebruik van herhaalde metingen van biomarkers en slagaderstijfheid (hoofdstuk 8).

In hoofdstuk 8 lieten we zien dat in 293 gezonde jonge volwassenen van het Amsterdamse Groei en Gezondheidsonderzoek endotheeldisfunctie en laaggradige ontsteking verband hielden met grotere slagaderstijfheid over een periode van 6 jaar. Endotheeldisfunctie hield verband met grotere stijfheid van de bovenbeenslagader. Laaggradige ontsteking hield verband met grotere stijfheid van de halsslagader en de bovenbeenslagader. Endotheeldisfunctie en laaggradige ontsteking waren echter niet 
gerelateerd aan grotere stijfheid van de grote lichaamsslagader. Omdat endotheeldisfunctie en laaggradige ontsteking nauw aan elkaar verwant zijn, voerden we aanvullende analyses uit die lieten zien dat de verbanden tussen endotheeldisfunctie stijfheid van de beenslagader en tussen laaggradige ontsteking en stijfheid van de beenslagader van elkaar afhankelijk waren. Het verband tussen laaggradige ontsteking en stijfheid van de halsslagader was echter onafhankelijk van endotheeldisfunctie. De beide verbanden tussen endotheeldisfunctie en laaggradige ontsteking en grotere stijfheid van de beenslagader werden voornamelijk gedreven door een afgenomen rekbaarheid (distentie) van de beenslagader. Het verband tussen laaggradige ontsteking en grotere stijfheid van de halsslagader werd niet gedreven door één van de gemeten slagader parameters, te weten diameter, distensie, lokale polsdruk (bloeddruk) en intima-media dikte (een maat voor vaatwanddikte).

In hoofdstuk 9 werd het bovenstaande onderzoek herhaald in een dwarsdoorsnede onderzoek in 601 oudere individuen van de Hoorn Studie met als focus stijfheid van de halsslagader. Voor endotheeldisfunctie werd nu, naast in bloedmonsters gemeten biomarkers, ook bloedstroomgemedieerde vaatwandverwijding als maat meegenomen. Laaggradige ontsteking hield verband met grotere stijfheid van de halsslagader, maar endotheeldisfunctie deed dit niet. Het verband tussen laaggradige ontsteking en grotere stijfheid van de halsslagader werd voornamelijk bepaald door een grotere diameter van de halsslagader.

\section{Interpretatie van de resultaten}

Nadat we uitgebreid de belangrijkste sterke en zwakke punten van onze studies uiteengezet hebben in hoofdstuk 10, concluderen we dat dit proefschrift bewijs levert dat een levenslang dagelijks gezond dieet, rijk aan vis, groenten en fruit, met matig gebruik van alcoholische dranken, bij uitstek (rode) wijn, en met magere in plaats van vette melkproducten, hart- en vaatziekten kan voorkómen en/of verminderen door een gunstige werking op endotheeldisfunctie en laaggradige ontsteking. Ondanks deze verbanden blijft het onderliggende mechanisme dat beschrijft hoe dieet endotheeldisfunctie en laaggradige ontsteking beïnvloedt echter onverklaard. Dit komt omdat een verband niet oorzakelijk hoeft te zijn. Onderzoek van anderen geeft hier echter een invulling aan. Waarschijnlijk spelen de volgende mechanismen een rol: het verbeteren van de stabiliteit van celmembranen; het remmen van de intracellulaire signalen die ontsteking activeren; het remmen van oxidatieve stress; het gunstig beïnvloeden van enzymen die endotheelcellen goed laten werken. Tezamen met onze bevindingen geven deze mechanismen bewijs hoe een gezond dieet endotheeldisfunctie en laaggradige ontsteking en daarmee de ontwikkeling van hart- en vaatziekten in mensen gunstig kan beïnvloeden.

Onze resultaten met betrekking tot slagaderstijfheid suggereren dat endotheeldisfunctie en laaggradige ontsteking slagaderstijfheid kunnen veroorzaken. Daarnaast 
lieten we zien dat endotheeldisfunctie en laaggradige ontsteking, voor een belangrijk deel, op een van elkaar afhankelijke manier verband houden met meer slagaderstijfheid. Dit geeft de nauwe relatie tussen endotheeldisfunctie en laaggradige ontsteking weer. Endotheeldisfunctie en laaggradige ontsteking kunnen tot grotere slagaderstijfheid leiden doordat ze een toename van de spier(cel)spanning in de slagaderwand veroorzaken en doordat ze veranderingen in de chemische stoffen die de spiercellen in slagaders laten ontspannen of samentrekken beïnvloeden. Daarnaast kunnen ze tot grotere slagaderstijfheid leiden via een toename van het aantal spiercellen en de grootte van spiercellen in de slagaderwand, een verschuiving van het evenwicht tussen elastische en starre eiwitten (elastine en collageen) in de slagaderwand en door het bevorderen van afzetten van calcium op elastische eiwitten in de slagaderwand. We concluderen dat onze resultaten samen met deze mechanismen de centrale doelstelling, dat endotheeldisfunctie en laaggradige ontsteking slagaderverstijving kunnen veroorzaken, ondersteunen.

\section{Betekenis van het onderzoek}

Dit proefschrift geeft bewijs dat een langdurig gezond dieet hart- en vaatziekten kan verminderen door een gunstige werking op endotheeldisfunctie en laaggradige ontsteking. De resultaten van de populatiestudies zijn belangrijk omdat ze inzicht geven hoe dagelijkse dieetgewoonten effect hebben op endotheeldisfunctie en laaggradige ontsteking en daarmee de ontwikkeling van hart- en vaatziekten. Deze resultaten zijn een aanvulling op gecontroleerd laboratorium onderzoek dat gedetailleerde informatie verschaft over biologische processen die een rol spelen bij endotheeldisfunctie en laaggradige ontsteking. Dit proefschrift is vooral belangrijk omdat het de resultaten van het onderzoek naar endotheeldisfunctie en laaggradige ontsteking vertaalt in een relevante gezondheidsboodschap voor de gehele populatie. Hoewel de gebruikte biomarkers van endotheeldisfunctie en laaggradige ontsteking (nog) geen rol spelen in patiëntenzorg geven de resultaten duidelijk aan dat artsen patiënten dienen te adviseren ten aanzien van een gezonde leefstijl en een dagelijks gezond dieet. Aanvullende analyses in de beschreven onderzoeken lieten zien dat een dagelijks gezond dieet wellicht het belangrijkst is in patiënten met chronische ziekten, zoals diabetes mellitus type 1 , diabetes mellitus type 2 en hart- en vaatziekten. Deze resultaten ondersteunen het recente internationale advies dat voedingsaanbevelingen geïntensiveerd dienen te worden in patiënten met zo'n chronische ziekte.

De implementatie van een dagelijks gezond dieet voor iedereen kan echter niet alleen de verantwoordelijkheid zijn van artsen. De resultaten in dit proefschrift werden in verschillende populaties verkregen en een gunstige invloed van een gezond dieet op endotheeldisfunctie en laaggradige ontsteking speelt dus waarschijnlijk een rol zowel in de vroege ontwikkeling alsook in het beloop van hart- en vaatziekten. Dit proefschrift ondersteunt daarmee de internationale visie dat gezondheids- 
bevorderende strategieën die de dieetgewoonten van individuen verbeteren geïntroduceerd dienen te worden op populatieniveau. Implementatie van een dagelijks gezond dieet dat gunstige effecten heeft op endotheeldisfunctie en laaggradige ontsteking is belangrijk voor de gehele populatie, omdat een kleine verschuiving in het risico op hart- en vaatziekten voor een individu door een gezond dieet op populatieniveau tot omvangrijke gezondheidswinst voor de hele samenleving kan leiden. 
Acknowledgements (dankwoord) 


\section{Dankwoord}

De afgelopen zes jaar heb ik met veel plezier wetenschappelijk onderzoek gedaan. De resultaten hiervan hebt $\mathrm{u}$ allemaal kunnen lezen in de voorgaande hoofdstukken. Ik bedoel ook echt "kunnen", want velen zullen na de "stellingen" direct doorgegaan zijn naar dit laatste hoofdstuk; het dankwoord. Een lastig hoofdstuk om te schrijven en niet onbelangrijk ook. Een proefschrift schrijven doe je namelijk niet alleen en ik ben velen mijn dank verschuldigd. Dit dankwoord beschrijft een poging iedereen die een bijdrage geleverd heeft te bedanken en daarmee begin ik bij de deelnemers aan de diverse studies. Hen ben ik dankbaar, want dit soort onderzoek is anders onmogelijk.

Bovenal wil ik mijn promotieteam bestaande uit dr. Ronald Henry, prof. dr. Coen Stehouwer en prof. dr. Casper Schalkwijk bedanken. Door jullie heb ik mezelf verder kunnen ontwikkelen op wetenschappelijk en persoonlijk vlak. Ik heb het ook als zeer prettig ervaren deel uit te maken van een door jullie gecreëerde groep enthousiaste onderzoekers.

Beste Ronald, ik wil je bedanken voor de dagelijkse begeleiding van mijn promotietraject. Jouw betrokkenheid, positieve houding en wetenschappelijke kwaliteiten hebben er mede voor gezorgd dat dit proefschrift er nu ligt. Ik heb onze samenwerking altijd als bijzonder prettig ervaren en heb ontzettend veel van je geleerd. Onze trips naar Wageningen waren altijd een welkome onderbreking van de dagelijks onderzoekswerkzaamheden en het was altijd gezellig op congres (EASD in Lissabon, Artery in Parijs). Zowel in het wetenschappelijk onderzoek als tijdens werkzaamheden in de kliniek staat zorgvuldigheid bij jouw altijd voorop. Ondanks drukte bewaak je het overzicht en blijf je altijd positief en constructief. Deze eigenschappen heb ik bijzonder in jouw gewaardeerd. Wat wetenschap betreft weet ik zeker dat er na mij nog vele succesvolle promovendi zullen volgen. Onze samenwerking gaat nog even door (10-jaren contract, toch?) en in de kliniek hoop ik nog meer van je te leren.

Beste Coen, ik ben trots dat jij mijn promotor bent en dat ik zoveel van jouw expertise uit de eerste hand heb mogen leren. Ik wil je vooral bedanken voor je scherpe en snelle commentaar, je betrouwbaarheid en je geduld om jouw redeneringen aan mij uit te leggen. Ik begrijp nog steeds niet hoe je zo snel, zo volledig een manuscript kunt becommentariëren, waarbij je zowel de kleine missers eruit haalt, alsook de grote lijn verbetert. Ik vond het altijd zeer prettig zo snel, zo'n goed commentaar te krijgen. Daarnaast ben ik je ook dankbaar voor de mogelijkheden die je bood zodat ik mezelf verder heb kunnen ontwikkelen. Ondanks je drukke agenda, en vele promovendi, hield je mijn ontwikkeling altijd goed in de gaten en stuurde waar nodig op tijd bij. Bedankt daarvoor! Ik hoop tijdens de opleiding tot internist opnieuw veel van je te leren. 
Beste Casper, ondanks dat je op papier mijn tweede promotor bent, ben je altijd zeer betrokken geweest gedurende het hele promotietraject. Bedankt! Op het laboratorium heerst onder jouw leiding een ontzettend goede (werk)sfeer en dit is voor een belangrijk deel aan jou te danken. Naast jouw uitgebreide wetenschappelijke kennis en kunde ben je namelijk altijd geïnteresseerd in anderen, ben je bereid te helpen en ben je in voor gezelligheid. Dit laatste bleek ook tijdens onze trip naar Orlando waar we lange, inhoudelijke congresdagen afwisselden met gezellige avonden in de kroeg. Ook zal ik (net als alle andere passagiers...) niet snel vergeten hoe hard wij moesten lachen om "the Hangover" in het vliegtuig terug naar Nederland. Ik wil ook graag jou en Hermien bedanken voor alle gastvrijheid bij jullie thuis. Hier hebben we met de hele groep vele leuke middagen en avonden doorgebracht tijdens de lab-uitjes en kerstdiners. Het werken als epidemiologie-Aio in een laboratorium omgeving heeft mijn wetenschappelijke blik verruimd. Bedankt voor alles!

De leden van beoordelingscommissie prof. dr. ir. R.P. Mensink (voorzitter), prof. dr. M.L. Bots, prof. dr. ir. P.C. Dagnelie, prof. dr. P.W. de Leeuw en prof. dr. N.C. Schaper wil ik hartelijk bedanken voor de aandacht die zij besteed hebben aan de beoordeling van dit proefschrift.

Marcelle en Yvo, bedankt dat jullie me bij willen staan als paranymfen. Jullie, samen met alle andere mede-Aios and post-docs, wil ik in het bijzonder bedanken voor alle keren dat jullie me er doorheen hielpen wanneer onderzoek tegen zat, voor de ontelbare discussies over wetenschap en andere belangrijke dingen in het leven, voor sportieve activiteiten, en voor de gezelligheid op het werk en tijdens de vele borrels en feestjes: daar gaan we, in willekeurige volgorde, maar dames eerst: Katrien, Johanna, Lian, Hanneke, Amy, José, Rianne, Marjon, Elisabeth, Pauline, Dionne, Olaf, Roel, Nick, Thomas, Nordin, Kristiaan, Matthijs, Barry en Dennis; bedankt voor de leuke tijd! Ik wens jullie allemaal heel veel succes met jullie promoties, en voor diegene die al zover zijn, veel succes in jullie verdere (wetenschappelijke) carrière!

Belangrijk waren nog vele andere mensen van onze afdeling. Beste Marjo, de combinatie van secuurheid en snelheid waarmee jij biomarkers meet is ongekend. Super Marjo! Het zelfstandig meten van biomarkers onder jouw supervisie gaf enorm veel inzicht. Bedankt! Marleen en Carla, bedankt voor jullie inhoudelijke bijdrage aan hoofdstukken 2 en 6 . Ik heb onze samenwerking altijd als zeer prettig ervaren. Beste Isabel, ik wil je bedanken voor je scherpe commentaar op de epidemiologie en statistiek en je inhoudelijke bijdrage aan diverse hoofdstukken in dit proefschrift. Petra, met jouw ervaring in de wetenschap kon je me vaak de weg wijzen. Bedankt! Boy, de discussies over microcirculatie waren altijd interessant en het stappen in Lissabon was ongekend! Vicky, Joséphine en Margee, bedankt voor de prettige samenwerking. Jean, jou wil ik graag bedanken voor je hulp bij de omslag van dit 
boekje. Ik ben er erg trots op dat het zo mooi geworden is! Ook ben ik je dankbaar voor alle gezelligheid die je meebrengt naar het werk! Tiny, bedankt voor je hulp bij de opmaak van dit boekje. Dit is goud waard op het einde van een promotietraject!

Het voedings-epi-centrum van Nederland is Wageningen en ik ben blij dat ik hier mijn kennis op voedingsgebied heb mogen verbreden en delen. Prof. dr. ir. Feskens, beste Edith, ik wil je graag bedanken voor je hulp en advies bij analyses van voedingsdata en daarmee je bijdrage aan de diverse hoofdstukken in het proefschrift. Martinette, Els en Truus, jullie wil ik bedanken voor het overleg over voedingsdata en de hulp bij het verwerken van het voedingsdagboek. Dit laatste heeft me veel geleerd over het meten van voeding. Dr. Soedamah-Muthu, beste Sabita, bedankt voor je bijdrage aan hoofdstuk 7. Ik vond het erg leuk om met je samen te werken.

De Amsterdamse AGGO en Hoorn onderzoekers dr. Fleur Schouten, dr. Michiel de Boer, prof. dr. Yvo Smulders, prof. dr. Jos Twisk, prof. dr. ir. Jacqueline Dekker en prof. dr. Giel Nijpels wil ik bedanken voor alle samenwerkingen die geresulteerd hebben in diverse hoofdstukken in dit proefschrift. Fleur, ik vond het erg leuk om hoofdstuk 8 samen met je te schrijven. Jos, tijdens het epidemiologie onderwijs aan het EMGO instituut heb ik veel van je geleerd en ook tijdens de laatste loodjes van het proefschrift heb ik veel aan jouw adviezen gehad. Bedankt! Jacqueline, jouw commentaar maakte de manuscripten altijd beter! Bedankt dat ik gebruik mocht maken van de AGGO en Hoorn data!

Uit Maastricht wil ik verder dr. ir. Koen Reesink, prof. dr. ir. Arnold Hoeks, Jos Op 't Roodt en dr. Floris Vanmolkot bedanken voor de bijdrage aan hoofdstuk 3. Koen en Jos, klinisch onderzoek doen met jullie is ontzettend leuk en ik heb zelf ervaren dat vaatfunctie niet met één druk op de knop te meten is. Arnold, onze wetenschappelijk discussies waren vaak diepgaand en ook heel verhelderend. Dank!

Dear prof. dr. Chaturvedi, prof. dr. Fuller and dr. Toeller, I would like to thank you for your support and contributions that have led to the manuscript described in chapter 7. Thank you for the opportunity to use the (dietary) data from the EURODIAB Study.

Ik wil graag de medewerkers van TIFN bedanken, in het bijzonder dr. Jolanda van Golde, (en eerder dr. Lisette Brink), projectleider(s) van project A-1004.

Natuurlijk zijn er ook vele anderen die (indirect) bijgedragen hebben aan mijn proefschrift. Mijn "nieuwe" collega's van de interne geneeskunde wil ik bedanken voor hun interesse in mijn promotieonderzoek. In het bijzonder wil ik dr. Evelien Pijpers, dr. Maya Huijberts en prof. dr. em. Arie Nieuwenhuijzen-Kruseman bedanken. Jullie maakten mij ruim 6 jaar geleden als student enthousiast voor het vak interne geneeskunde en voor wetenschappelijk onderzoek. Zie hier het resultaat! 
Ik wil graag mijn familie en vrienden bedanken voor hun belangstelling in mijn promotieonderzoek. Jullie waren belangrijk gedurende de hele rit want zonder de vele feestjes, concerten, voetbalwedstrijden, etentjes, fiets- en schaatstochten, wintersportvakanties, weekendjes, borrels en stapavonden had ik het waarschijnlijk niet volgehouden. Dank hiervoor!

Iris en Hans, Etienne en Evelyne, Jordi en Shannon, jullie wil ik bedanken voor jullie belangstelling, en natuurlijk voor alle gezellige etentjes en de fanatieke spelletjes avonden! Deze waren altijd een welkome onderbreking.

Frank, samen lukt ons een heleboel, zoals voetballen, op stap gaan en zelfs wetenschap (zie hoofdstuk 3)! En dit alles waardeer ik enorm. Hanke, voor mij is het prettig dat Frank in Maastricht woont, maar voor jullie is Maastricht Utrecht toch iedere keer weer een lange weg. Ondanks dat zien we elkaar regelmatig en dat is altijd erg gezellig. Bedankt voor jullie interesse in mijn promotieonderzoek. Ik wens jullie ook veel succes met jullie promotieonderzoeken! Mark, ons familiewetenschappelijk hoogtepunt blijft toch het abstract met ons drieën erop (met dank aan Koen R.)! Jij bent verreweg mijn trouwste lezer. Ik vind het ook mooi hoe je mij altijd wijst op de nieuwste stand van zaken met betrekking tot de pillen en de poeders.

Pap en Mam, jullie ben ik het meest dankbaar van iedereen. Het is thuis altijd gezellig en leuk. Jullie hebben me (ons) altijd gestimuleerd en gesteund om nieuwe uitdagingen in het leven aan te gaan, om vrienden te maken, om te studeren en om te reizen. Bedankt voor alles!

Ivette, ik hoop dat ik jou op dezelfde manier kan steunen met jouw promotieonderzoek. Jij bent er altijd voor me en dat geeft een goed gevoel. Ik hou van jou! 
Curriculum vitae

245 
CV 


\section{Curriculum vitae}

Bas Carolus Theodorus van Bussel was born on 02-02-1983 in Asten-Heusden, the Netherlands. He went to secondary school at the St. Willibrord Gymnasium, Deurne, the Netherlands, and graduated in 2001. He spent a gap year working and travelling around Australia, New Zealand and Indonesia. In 2002, Bas started to study Medicine at the Faculty of Health, Medicine and Life Sciences of the Maastricht University Medical Center, Maastricht, the Netherlands. He obtained his medical degree in August 2008. In May 2008, he started to work as a PhD student at the department of Internal Medicine and the School for Nutrition, Toxicology and Metabolism (NUTRIM) of the Maastricht University Medical Center, Maastricht, the Netherlands. The research was done within the framework of Top Institute Food and Nutrition (TIFN), Wageningen, the Netherlands, as part of the project "Diet and early endothelial dysfunction". In 2010 Bas obtained his Master degree in Epidemiology at the EMGO institute for Health and Care Research, Vrije Universiteit, Amsterdam, the Netherlands. The results of Bas' research are presented in this thesis, which was performed under the supervision of promotores prof. dr. Coen Stehouwer and prof. dr. Casper Schalkwijk, and copromotor dr. Ronald Henry. In October 2012 Bas has started his training to become an Internist under the supervision of prof. dr. Coen Stehouwer (chair) and prof. dr. Richard Koopmans (acting trainer) at the department of Internal Medicine of the Maastricht University Medical Center, Maastricht, the Netherlands, where he is currently working. 
CV 
List of publications 


\section{List of publications}

van Bussel BC, Henry RM, Schalkwijk CG, Ferreira I, Dekker JM, Nijpels G, Feskens EJ, Stehouwer $\mathrm{CD}$. Alcohol and red wine consumption are associated with less endothelial dysfunction and less low-grade inflammation: the Hoorn Study. In preparation

van Bussel BC, Henry RM, Ferreira I, van Greevenbroek MM, van der Kallen CJ, Twisk JW, Feskens EJ, Schalkwijk CG, Stehouwer CD. A healthy diet is associated with less endothelial dysfunction and less low-grade inflammation over a 7-year period: the CODAM Study. Submitted

van Bussel FC*, van Bussel BC*, Hoeks AP, Op 't Roodt J, Henry RM, Ferreira I, Vanmolkot FH, Schalkwijk CG, Stehouwer CD, Reesink KD. Control systems approach to assessment of flow-mediated dilation improves the detection of changes in local brachial artery dilation after an oral glucose load. Submitted

van Bussel BC, Ferreira I, van de Waarenburg MP, van Greevenbroek MM, van der Kallen CJ, Henry RM, Feskens EJ, Stehouwer CD, Schalkwijk CG. Multiple inflammatory biomarker detection in a prospective cohort study: a cross-validation between wellestablished single-biomarker techniques and an electrochemiluminescense-based multi-array platform. PLoS One. 2013;8:e58576.

van de Laar RJ, Stehouwer CD, van Bussel BC, Prins MH, Twisk JW, Ferreira I. Adherence to a Mediterranean dietary pattern in early life is associated with lower arterial stiffness in adulthood: the Amsterdam Growth and Health Longitudinal Study. J Intern Med. 2013;273:79-93.

van Bussel BC, Soedamah-Muthu SS, Henry RM, Schalkwijk CG, Ferreira I, Chaturvedi $\mathrm{N}$, Toeller M, Fuller JH, Stehouwer CD; EURODIAB Prospective Complications Study Group. Unhealthy dietary patterns associated with inflammation and endothelial dysfunction in type 1 diabetes: the EURODIAB study. Nutr Metab Cardiovasc Dis. 2013;23:758-64.

van de Laar RJ, Stehouwer CD, van Bussel BC, te Velde SJ, Prins MH, Twisk JW, Ferreira I. Lower lifetime dietary fiber intake is associated with carotid artery stiffness: the Amsterdam Growth and Health Longitudinal Study. Am J Clin Nutr. 2012;96:14-23.

van Bussel BC, Henry RM, Schalkwijk CG, Dekker JM, Nijpels G, Stehouwer CD. Lowgrade inflammation, but not endothelial dysfunction, is associated with greater carotid stiffness in the elderly: the Hoorn Study. J Hypertens. 2012;30:744-52. 
van Bussel BC*, Schouten F*, Henry RM, Schalkwijk CG, de Boer MR, Ferreira I, Smulders YM, Twisk JW, Stehouwer CD. Endothelial dysfunction and low-grade inflammation are associated with greater arterial stiffness over a 6-year period. Hypertension. 2011;58:588-95.

van Bussel BC, Henry RM, Schalkwijk CG, Ferreira I, Feskens EJ, Streppel MT, Smulders YM, Twisk JW, Stehouwer CD. Fish consumption in healthy adults is associated with decreased circulating biomarkers of endothelial dysfunction and inflammation during a 6-year follow-up. J Nutr. 2011;141:1719-25.

van Bussel B, Pijpers E, Ferreira I, Castermans P, Kruseman AN. Polymorbidity in diabetes in older people: consequences for care and vocational training. Postgrad Med J. 2007;83:763-7.

* both authors contributed equally

\section{Oral presentations}

Nederlandse Vereniging voor Hart en Vaat Verpleegkundigen (NVHVV), Utrecht, the Netherlands, March 2012

European Association for the Study of Diabetes (EASD), Lisbon, Portugal, September 2011

Werkgroep Epidemiologisch Onderzoek Nederland (WEON), Netherlands Epidemiology Society, IJmuiden, the Netherlands, June 2011

Anglo-Danish-Dutch Diabetes Group, Perthshire, Scotland, April 2011

Werkgroep Epidemiologisch Onderzoek Nederland (WEON), Netherlands Epidemiology Society, Nijmegen, the Netherlands, June 2010

Nederlandse Vereniging voor Hart en Vaat Verpleegkundigen (NVHVV), Utrecht, the Netherlands, March 2010

Internistendagen, Nederlandse Internisten Vereniging (NIV), Maastricht, the Netherlands, April 2009

Artery 8, Artery Society, Ghent, Belgium, September 2008 


\section{Poster presentations}

Artery 11, Artery Society, Paris, France, October 2011

Artery 10, Artery Society, Verona, Italy, October 2010

American Heart Association Scientific Sessions, Orlando, USA, October 2009

Werkgroep Epidemiologisch Onderzoek Nederland (WEON), Netherlands Epidemiology Society, Amsterdam, the Netherlands, June 2009

European Federation of Internal Medicine (EFIM) Conference, Lisbon, Portugal, 2007 
